\title{
Ti-Catalyzed Reductive Dehydroxylative Vinylation of Tertiary Alcohols
}

Hao Xie, Sheng Wang, Yuquan Wang, Peng Guo, Xing-Zhong Shu*

State Key Laboratory of Applied Organic Chemistry (SKLAOC), College of Chemistry and Chemical Engineering, Lanzhou University, 222 South Tianshui Road, Lanzhou, 730000, China.

Email: shuxingzh@lzu.edu.cn

\section{Table of Contents}

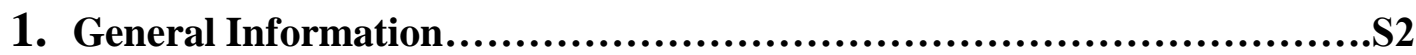

2. Optimization of Reaction Conditions (Table S1)............................S3

3. Preparation of Starting Materials............................................S5

4. Ti-Catalyzed Stereoconvergent Radical Dehydroxylative Vinylation of Tertiary Alcohols with Vinyl Halides....................................S19

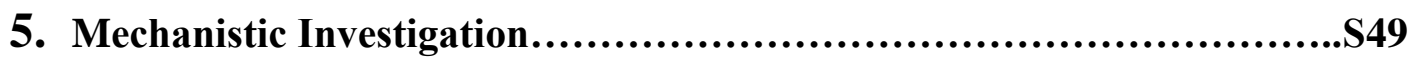

6. References...................................................................S52

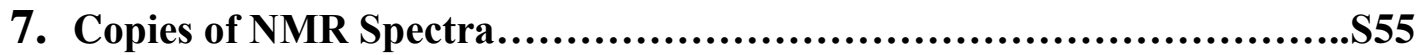




\section{General Information}

All reactions were carried out under an atmosphere of argon in a sealed tube with magnetic stirring. Dry THF, DMF, DMA, Toluene, $\mathrm{CH}_{3} \mathrm{CN}$ were purified using a solvent-purification system that contained activated alumina and molecular sieves. Other solvents were dried and purified according to the procedure from "Purification of Laboratory Chemicals". ${ }^{1}$

Titanium catalysts, reductants were purchased from J\&K, Alfa Aesar. Other chemicals were purchased from TCI, Adamas, Acros, and Energy chemicals, and they were used directly without further purifications.

${ }^{1} \mathrm{H}$ and ${ }^{13} \mathrm{C}$ NMR spectra were collected on a Bruker AVANCE III $400 \mathrm{MHz}$, JEOL JNM-ECS 400M, and Agilent-NMR-Inova $600 \mathrm{MHz}$ spectrometer at room temperature. ${ }^{1} \mathrm{H}$ NMR spectra were reported in parts per million (ppm) downfield of tetramethylsilane (TMS) and were referenced to the signal of TMS (0 ppm). ${ }^{13} \mathrm{C}$ NMR spectra were reported in ppm relative to residual $\mathrm{CHCl}_{3}(77.00 \mathrm{ppm})$. Coupling constants, $J$, are reported in hertz $(\mathrm{Hz}) .{ }^{19} \mathrm{~F}$ NMR spectra were collected on Bruker AVANCE III 400 MHz spectrometers and Agilent-NMR-Inova 600 MHz spectrometer at room temperature. IR spectra were collected using Bruker-TENSOR 27 spectrometer and Agilent Technologies Cary 630 FTIR, and only major peaks were reported in $\mathrm{cm}^{-1}$. HRMS was performed on Bruker Apex II FT-ICR mass instrument (ESI). GC analysis was performed on Thermo Scientific TRACE 1300. GC-MS data were collected on Thermo Scientific TRACE DSQ GC-MS. Thin-layer chromatography was carried out using XINNUO SGF254 TLC plates. Flash chromatography was performed using XINNUO silica gel (200-300 mesh). 


\section{Optimization of Reaction Conditions}

General Procedure A.The procedure was conducted in an argon-filled glove box. To a reaction tube equipped with a magnetic stir bar was charged with catalyst (10 mol \%, $0.02 \mathrm{mmol})$, molecular sieve $(20 \mathrm{mg})$, reductant (3 equiv, $0.6 \mathrm{mmol})$, 2-methyl-4phenylbutan-2-ol 1a (32.8 mg, $0.2 \mathrm{mmol})$, (E)-(2-bromovinyl)benzene 2a (65.5 mg, $0.36 \mathrm{mmol})$, solvent $(0.4 \mathrm{~mL})$ and chlorosilane (3 equiv, $0.6 \mathrm{mmol})$. The reaction tube was sealed with a rubber septum and removed from the glove box. The reaction mixture was stirred at appreciate temperature for $12 \mathrm{~h}$. The reaction mixture was diluted with ethyl acetate $(10 \mathrm{~mL})$, washed with water, brine, and dried over anhydrous $\mathrm{Na}_{2} \mathrm{SO}_{4}$. A $0.2 \mathrm{~mL}$ of solution was collected, diluted with ethyl acetate $(2 \mathrm{~mL})$, and analyzed by GC. The yield was determined versus the internal standard (hexadecane).

Optimization of the reaction conditions. We started our investigation by studying the reaction of alcohol 1a and vinyl bromide 2a (Table S1). We found the combination of $\mathrm{Cp}^{*} \mathrm{TiCl}_{3}$, TESCl, and $\mathrm{Zn}$ delivered vinylated product $\mathbf{3 a}$ in $86 \%$ isolated yield in 0.2 mmol scale and $73 \%$ yield $(0.9 \mathrm{~g})$ in gram scale (entry 1$)$. The reaction efficiency is significantly affected by the electronic factor of the titanium catalyst. $\mathrm{CpTiCl}_{3}$ $\left[\mathrm{E}_{1 / 2}(\mathrm{IV} / \mathrm{III})=-0.82 \mathrm{~V}\right]$, which is less reducing than $\mathrm{Cp}^{*} \mathrm{TiCl}_{3}\left[\mathrm{E}_{1 / 2}(\mathrm{IV} / \mathrm{III})=-1.12 \mathrm{~V}\right]$, gave 3a in a low yield with large amount of alcohol 1a being recovered (entry 2). The same was also true of $\mathrm{Cp}_{2} \mathrm{TiCl}_{2}$ (entries 3 vs 4 ). The sterically hindered $\mathrm{Cp}^{*}{ }_{2} \mathrm{TiCl}_{2}$ decreased the yield to $63 \%$ (entries 1 vs 4). Large sterically hindered TBSCl resulted in a trace of 3a and recovery of alcohol 1a, whereas the use of TMSCl gave 3a in $66 \%$ yield (entries 5 and 6). The substrate of tertiary alcohols appears to be acid-sensitive; the use of $\mathrm{Et}_{3} \mathrm{~N} \cdot \mathrm{HCl}$ or Coll $\cdot \mathrm{HCl}$ only delivered the dehydration product (entries 7 and 8). The reaction at $30{ }^{\circ} \mathrm{C}$ afforded $\mathbf{3 a}$ in a low yield and no desired product was obtained when Mn was used (entries 9 and 10). The use of $5 \AA$ MS slightly increased the yield from $69 \%$ to $78 \%$ (entries 1 and 11). No or trace of $\mathbf{3 a}$ was observed in the absence of TESCl, Ni, or Zn (entries 12 and 13). 
Table S1. Optimization of the Reaction Conditions ${ }^{a}$

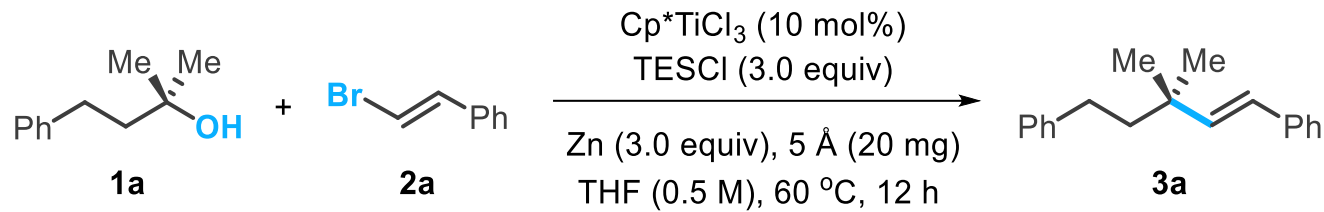

\begin{tabular}{lll}
\hline entry & change of conditions & $\mathbf{3 a}(\%)$ \\
\hline 1 & None & $78 \%(86 \%)^{b}$ \\
2 & $\mathrm{CpTiCl}_{3}$ & 38 \\
3 & $\mathrm{Cp}_{2} \mathrm{TiCl}_{2}$ & 43 \\
4 & $\mathrm{Cp}^{*}{ }_{2} \mathrm{TiCl}_{2}$ & 63 \\
5 & $\mathrm{TBSCl}^{2}$ & Trace \\
6 & $\mathrm{TMSCl}^{2}$ & 66 \\
7 & $\mathrm{Et}{ }_{3} \mathrm{~N} \cdot \mathrm{HCl}$ & 0 \\
8 & $\mathrm{Coll} \cdot \mathrm{HCl}$ & 0 \\
9 & $30{ }^{\circ} \mathrm{C}$ & 9 \\
10 & $\mathrm{Mn}$ & 0 \\
11 & $\mathrm{No} 5 \AA$ molecular sieves & 69 \\
12 & No TESCl & trace \\
13 & No Ti or Zn & 0
\end{tabular}

${ }^{a} \mathbf{1 a}(0.2 \mathrm{mmol})$ and $\mathbf{2 a}$ (1.8 equiv) were used; the yields were determined by GC analysis with hexadecane as internal standard. ${ }^{b}$ Isolated yield was given. 


\section{Preparation of Starting Materials}

\subsection{Synthesis of Tertiary Alcohols}

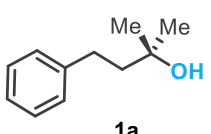

$1 \mathrm{a}$

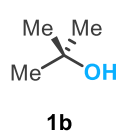

$1 \mathrm{~b}$

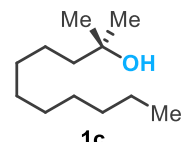

$1 \mathrm{c}$

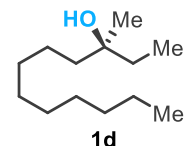

$1 d$

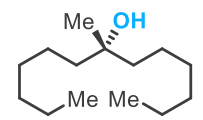

1e<smiles>CC=CC=CCC(C)(C)O</smiles>

$1 f$<smiles>CCOC(=O)CCCCC(C)(C)O</smiles><smiles>CC(C)(O)CCc1ccc(F)cc1</smiles><smiles>CC(C)(O)CCc1ccc2c(c1)OCO2</smiles><smiles>CC(C)(O)CCc1ccc(CCc2ccc([N+](=O)[O-])cc2)cc1</smiles><smiles>CC(C)(O)CCc1ccc(O)cc1</smiles><smiles>CC(C)(O)CCc1ccc(O)cc1</smiles><smiles>CC(C)(O)CCc1ccc(Br)cc1</smiles><smiles>CC(C)(O)CCCl</smiles><smiles>CC(O)c1ccccc1</smiles><smiles>CC1(O)CCOCC1</smiles>

$1 \mathrm{r}$<smiles>CC1(O)CCCCC1</smiles>

1s

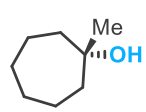

$1 \mathrm{t}$

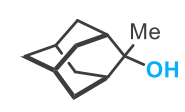

$1 \mathrm{u}$

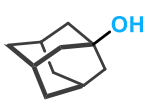

1v
$\underbrace{\mathrm{Me}}_{\mathrm{OH}}$

$1 w \quad 1 x$<smiles>CCCCCC[C@H](O)C/C=C\CCCCCCCCC(C)(C)O</smiles><smiles>CC(O)C(CO)Oc1ccc(CCC(C)(C)O)cc1</smiles>

Compounds 1a, 1b, 1p, 1q, 1r, 1s, 1t, and 1v are commercially available. Compounds 1c, 1d, 1e, 1g, 1h, 1i, 1j, 1k, 1l, 1m, 1n, 1o, 1u, 1w, 1x, 1y, and $1 \mathrm{z}$ are known compounds. ${ }^{2}$ The preparation of new compounds, and their characterization data are provided as follows.

\section{General Procedure B:}

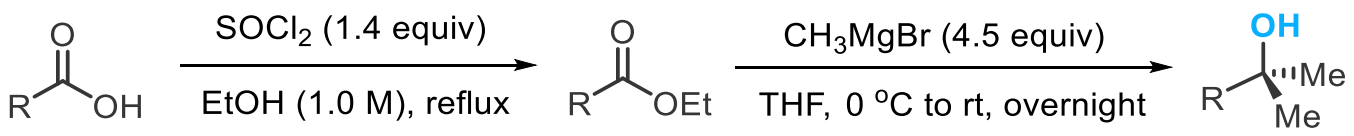

To a solution of acid $(10 \mathrm{mmol})$ in $\mathrm{EtOH}(10 \mathrm{~mL})$ was added $\mathrm{SOCl}_{2}(1.65 \mathrm{~g}, 14 \mathrm{mmol}$, 1.4 equiv) slowly. The reaction mixture was refluxed for $3 \mathrm{~h}$, and the solvent was removed under the reduced pressure. The residue was diluted with ethyl acetate $(30 \mathrm{~mL})$ 
and quenched with water $(20 \mathrm{~mL})$. The saturated aqueous solution of $\mathrm{NaOH}$ was added to adjust the $\mathrm{pH}$ to $8-9$. The organic layer was separated, washed with water and brine, dried over anhydrous $\mathrm{Na}_{2} \mathrm{SO}_{4}$, and concentrated under the reduced pressure. The residue was used directly for next step without further purification.

To a solution of the above residue in anhydrous THF $(20 \mathrm{~mL})$ was dropwise added a solution of $\mathrm{CH}_{3} \mathrm{MgBr}$ in $\mathrm{THF}(15 \mathrm{~mL}, 45 \mathrm{mmol}, 3 \mathrm{M})$ at $0{ }^{\circ} \mathrm{C}$. The reaction mixture was stirred at room temperature for overnight. The reaction was quenched with an aqueous solution of $\mathrm{NH}_{4} \mathrm{Cl}$ at $0{ }^{\circ} \mathrm{C}$, and extracted with ethyl acetate $(30 \mathrm{~mL} \times 2)$. The combined organic layer was washed with brine, dried over anhydrous $\mathrm{Na}_{2} \mathrm{SO}_{4}$, filtered, and concentrated in vacuum. The residue was purified by flash chromatography on silica gel to give the resulting alcohol.

(10Z, 13Z)-2-methylnonadeca-10,13-dien-2-ol (1f)

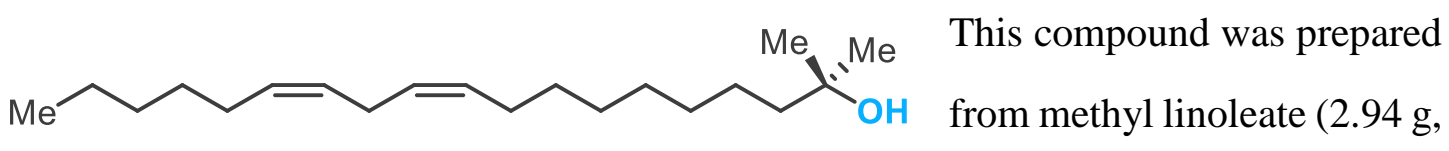

$10.0 \mathrm{mmol}$ ) according to the General procedure B (step 2).

$1.71 \mathrm{~g}(58 \%$ yield $)$, colorless oil.

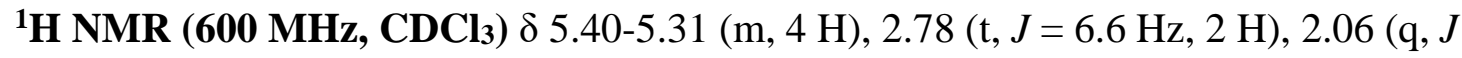
$=7.2 \mathrm{~Hz}, 4 \mathrm{H}), 1.47-1.31(\mathrm{~m}, 19 \mathrm{H}), 1.21(\mathrm{~s}, 6 \mathrm{H}), 0.89(\mathrm{t}, J=7.2 \mathrm{~Hz}, 3 \mathrm{H})$,

${ }^{13}$ C NMR (150 MHz, CDCl3) $\delta$ 130.2, 130.1, 128.0, 127.9, 71.0, 44.0, 31.5, 30.2, 29.7, $29.5,29.4,29.3,29.2,27.3,27.2,25.6,24.3,22.6,14.1$.

IR (neat, $\mathbf{c m}^{-1}$ ): 3015, 2932, 2857, 1655, 1459, 1292, 1248, 945, 716.

HRMS (ESI): $[\mathrm{M}+\mathrm{Na}]^{+}$calcd for $\mathrm{C}_{20} \mathrm{H}_{38} \mathrm{NaO} 317.2815$, found 317.2819.

\section{(R, Z)-2-methylnonadec-10-ene-2,13-diol (1aa)}

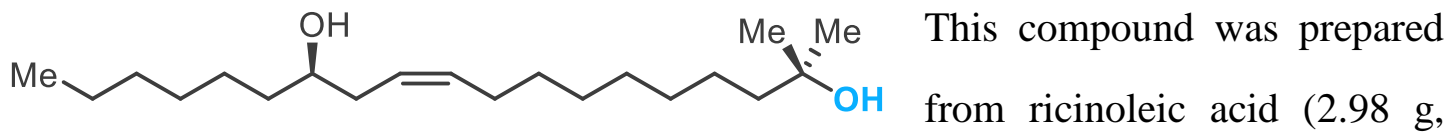

$10.0 \mathrm{mmol}$ ) according to the General procedure B.

$2.09 \mathrm{~g},(67 \%$ yield for two steps), colorless oil.

${ }^{1}$ H NMR (600 MHz, CDCl $) \delta$ 5.60-5.53 (m, 1 H), 5.44-5.37 (m, 1 H), 3.63-3.58 (m, $1 \mathrm{H}), 2.21(\mathrm{t}, J=9.8 \mathrm{~Hz}, 2 \mathrm{H}), 2.08-2.03(\mathrm{~m}, 2 \mathrm{H}), 1.50-1.44(\mathrm{~m}, 7 \mathrm{H}), 1.35-1.26(\mathrm{~m}$, 
$17 \mathrm{H}), 1.21$ (s, $6 \mathrm{H}), 0.89$ (t, $J=10.8 \mathrm{~Hz}, 3 \mathrm{H})$.

${ }^{13}$ C NMR (150 MHz, CDCl $) \delta$ 133.4, 125.2, 71.5, 71.0, 44.0, 36.8, 35.3, 31.8, 30.1, 29.6, 29.5, 29.3, 29.2, 29.1, 27.4, 25.7, 24.3, 22.6, 14.1.

IR (neat, $\mathbf{c m}^{-1}$ ): 2932, 2855, 1467, 1377, 1403, 1148, 1077, 1047, 980, 908, 731.

HRMS (ESI): [M+Na] ${ }^{+}$calcd for $\mathrm{C}_{20} \mathrm{H}_{40} \mathrm{NaO}_{2} 335.2921$, found 335.2920.

2-(4-(3-Hydroxy-3-methylbutyl)phenoxy)propane-1,3-diol (1ab)
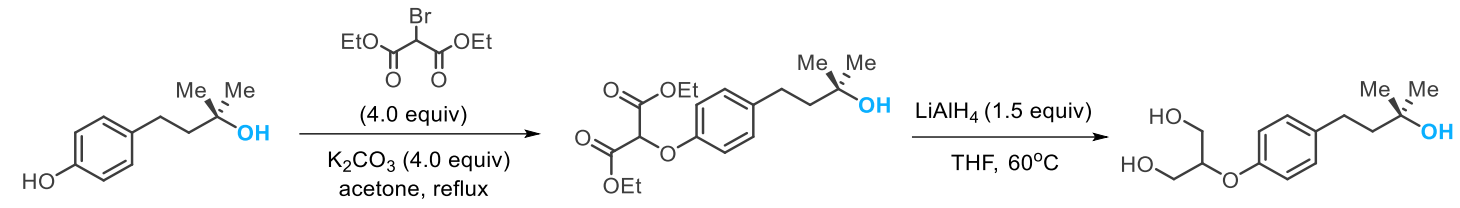

Step 1: To a solution of 4-(3-hydroxy-3-methylbutyl)phenol $(1.80 \mathrm{~g}, 10 \mathrm{mmol})$ and $\mathrm{K}_{2} \mathrm{CO}_{3}(5.52 \mathrm{~g}, 40 \mathrm{mmol})$ in acetone $(60 \mathrm{~mL})$ was added diethyl 2-bromomalonate $(9.52$ $\mathrm{g}, 40 \mathrm{mmol}$ ). The reaction mixture was refluxed for overnight. The reaction was quenched with water $(30 \mathrm{~mL})$, and the mixture was extracted twice with ethyl acetate (30 mL). The combined organic layers were washed with brine, dried over anhydrous $\mathrm{Na}_{2} \mathrm{SO}_{4}$, filtered, and concentrated to afford a crude product.

Step 2: To a stirred solution of the above residue (2.71 g, about $8 \mathrm{mmol})$ in THF (40 $\mathrm{mL})$ at $0{ }^{\circ} \mathrm{C}$ was added $\mathrm{LiAlH}_{4}(0.46 \mathrm{~g}, 12 \mathrm{mmol})$. The reaction mixture was stirred at $60{ }^{\circ} \mathrm{C}$ for overnight. The reaction was quenched with water $(20 \mathrm{~mL})$ and the mixture was extracted twice with EtOAc $(30 \mathrm{~mL})$. The combine organic layers were washed with brine, dried over anhydrous $\mathrm{Na}_{2} \mathrm{SO}_{4}$, filtered, and evaporated to dryness. The residue was purified by flash chromatography on silica gel to give 2-(4-(3-hydroxy-3methylbutyl)phenoxy)propane-1,3-diol.

$1.68 \mathrm{~g},\left(66 \%\right.$ yield for two steps), solid, mp. $78.2-80.1^{\circ} \mathrm{C}$

${ }^{1}$ H NMR (400 MHz, MeOD) $\delta 7.10(\mathrm{~d}, J=8.4 \mathrm{~Hz}, 2 \mathrm{H}), 6.92(\mathrm{~d}, J=8.4 \mathrm{~Hz}, 2 \mathrm{H})$, 4.32-4.27 (m, $1 \mathrm{H}), 3.79-3.71$ (m, $4 \mathrm{H}), 2.63-2.59$ (m, $2 \mathrm{H}), 1.73-1.69$ (m, $2 \mathrm{H}), 1.24$ (s, $6 \mathrm{H})$.

${ }^{13}$ C NMR (100 MHz, MeOD) $\delta$ 158.0, 137.0, 130.4, 117.5, 81.1, 71.4, 62.2, 47.3, 31.1, 29.3

IR (neat, $\mathbf{c m}^{-1}$ ): 2969, 2939, 2361, 2345, 1616, 1511, 1456, 1381, 1236, 1044, 978, 
$928,842$.

HRMS (ESI): [M+Na $]^{+}$calcd for $\mathrm{C}_{14} \mathrm{H}_{22} \mathrm{NaO}_{4}$ 277.1410, found 277.1407.

\section{2-(4-(3-Hydroxy-3-methylbutyl)phenoxy)butane-1,3-diol (1ac)}

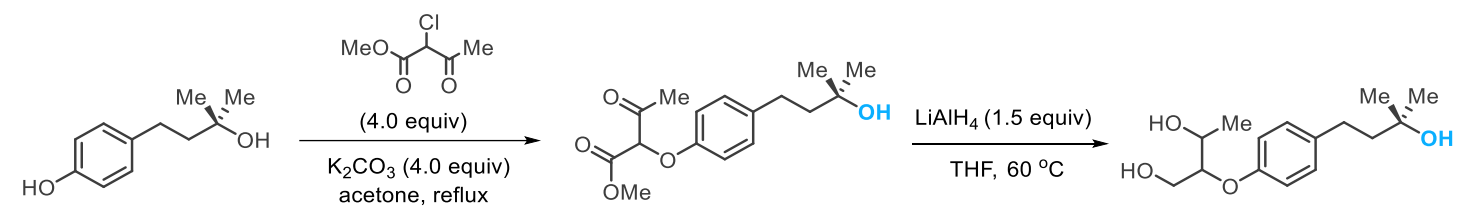

Step 1: To a solution of 4-(3-hydroxy-3-methylbutyl)phenol (1.80 g, $10 \mathrm{mmol})$ and $\mathrm{K}_{2} \mathrm{CO}_{3}(5.52 \mathrm{~g}, 40 \mathrm{mmol})$ in acetone $(60 \mathrm{~mL})$ was added methyl 2-chloro-3oxobutanoate $(6.00 \mathrm{~g}, 40 \mathrm{mmol})$. The reaction mixture was refluxed for overnight. The reaction was quenched with water $(40 \mathrm{~mL})$, and the mixture was extracted twice with ethyl acetate $(40 \mathrm{~mL})$. The combined organic layers were washed with brine, dried over anhydrous $\mathrm{Na}_{2} \mathrm{SO}_{4}$, filtered, and evaporated in vacuum to afford a crude product.

Step 2: To a stirred solution of the above residue (1.47 g, about $5 \mathrm{mmol})$ in THF (25 $\mathrm{mL})$ at $0{ }^{\circ} \mathrm{C}$ was added $\mathrm{LiAlH}_{4}(0.29 \mathrm{~g}, 7.5 \mathrm{mmol})$. The reaction mixture was stirred at $60{ }^{\circ} \mathrm{C}$ for overnight. The reaction was quenched with water $(20 \mathrm{~mL})$, and the mixture was extracted twice with EtOAc $(30 \mathrm{~mL})$. The combine organic layers were washed with brine, dried over anhydrous $\mathrm{Na}_{2} \mathrm{SO}_{4}$, filtered, and evaporated to remove the solvent. The residue was purified by flash chromatography on silica gel to give 2-(4-(3-hydroxy3-methylbutyl)phenoxy)butane-1,3-diol.

$1.10 \mathrm{~g}$, (41\% yield for two steps), colorless oil.

${ }^{1}$ H NMR (400 MHz, MeOD) $\delta 7.08(\mathrm{~d}, J=8.4 \mathrm{~Hz}, 2 \mathrm{H}), 6.94-6-90$ (m, $\left.2 \mathrm{H}\right), 4.11$ (q, $J=4.8 \mathrm{~Hz}, 1 \mathrm{H}), 4.06-3.97$ (m, $1 \mathrm{H}), 3.84-3.69$ (m, $2 \mathrm{H}), 2.62-2.58$ (m, $2 \mathrm{H}), 1.72-1.68$ $(\mathrm{m}, 2 \mathrm{H}), 1.23(\mathrm{~d}, J=13.2 \mathrm{~Hz}, 3 \mathrm{H}), 1.23(\mathrm{~s}, 6 \mathrm{H})$,

${ }^{13}$ C NMR (100 MHz, MeOD) $\delta$ 157.0, 156.9, 135.4, 128.8, 116.0, 82.9, 82.5, 69.9, $66.6,66.3,60.2,45.8,29.5,27.8,17.9,17.7$.

IR (neat, $\mathbf{c m}^{-1}$ ): 3383, 2971, 2932, 1724, 1612, 1463, 1511, 1463, 1379, 1297, 1239, 1177, 1097, 1407, 909, 833.

HRMS (ESI): [M+Na ${ }^{+}$calcd for $\mathrm{C}_{15} \mathrm{H}_{24} \mathrm{NaO}_{4} 291.1567$, found 291.1565 . 


\section{(7S, 8R)-15-methylhexadecane-1,7,8,16-tetraol (1ad)}<smiles>CC(C)(O)CCCCCCC[C@H](O)[C@H](O)CCCCCCO</smiles>

This compound was prepared from aleuritic acid $(3.04 \mathrm{~g}, 10.0 \mathrm{mmol})$ according to the General procedure B.

$2.26 \mathrm{~g}$, (71\% yield for two steps), solid, mp. 47.0-48.0 ${ }^{\circ} \mathrm{C}$.

${ }^{1} \mathbf{H}$ NMR (600 MHz, CDCl$) \delta 3.64(\mathrm{t}, J=10.2 \mathrm{~Hz}, 2 \mathrm{H}), 3.40(\mathrm{~s}, 2 \mathrm{H}), 1.59-1.25(\mathrm{~m}$, $28 \mathrm{H}), 1.21(\mathrm{~s}, 6 \mathrm{H})$.

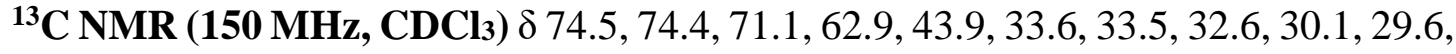
$29.5,29.4,29.3,29.2,25.7,25.6,25.5,24.3$.

IR (neat, $\mathbf{c m}^{-1}$ ): 2935, 2861, 1467, 1377, 1077, 1049, 982, 911, 800, 734.

HRMS (ESI): [M+Na] ${ }^{+}$calcd for $\mathrm{C}_{18} \mathrm{H}_{38} \mathrm{NaO}_{4} 341.2662$, found 341.2660.

\section{4-(4-(Hydroxymethyl)phenyl)-2-methylbutan-2-ol (1ae)}

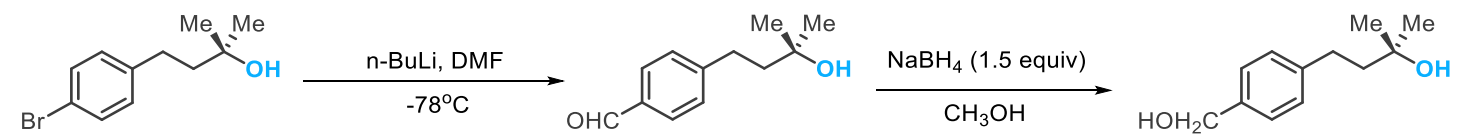

Step 1: To a solution of 4-(4-bromophenyl)-2-methylbutan-2-ol (2.42 g, $10.0 \mathrm{mmol})$ in THF (40 mL) was dropwise added $n$-BuLi (7.2 mL, 18.0 mmol, 2.5 M hexane solution) at $-78{ }^{\circ} \mathrm{C}$. After stirring at $-78{ }^{\circ} \mathrm{C}$ for $60 \mathrm{~min}$, DMF $(7.3 \mathrm{~g}, 100.0 \mathrm{mmol})$ was then dropwise added. After stirring at room temperature for overnight, the reaction was quenched with water $(30 \mathrm{~mL})$ and the mixture was extracted twice with EtOAc $(30 \mathrm{~mL})$. The combine organic layers were washed with brine, dried over anhydrous $\mathrm{Na}_{2} \mathrm{SO}_{4}$, filtered, and concentrated in vacuum to afford an aldehyde.

Step 2: To a stirred solution of the above residue (1.54 g, about $8 \mathrm{mmol})$ in $\mathrm{MeOH}(20$ $\mathrm{mL})$ at $0{ }^{\circ} \mathrm{C}$ was added $\mathrm{NaBH}_{4}(0.46 \mathrm{~g}, 12 \mathrm{mmol})$. The reaction mixture was allowed to warm to room temperature and stirred for $1 \mathrm{~h}$. Water $(50 \mathrm{~mL})$ was added and the mixture was extracted with $\mathrm{CH}_{2} \mathrm{Cl}_{2}(2 \times 30 \mathrm{~mL})$. The combined organic layers were washed with brine, dried over $\mathrm{Na}_{2} \mathrm{SO}_{4}$, filtered, and concentrated in vacuum. The residue was purified by flash chromatography on silica gel to give 4-(4-(hydroxymethyl)phenyl)-2methylbutan-2-ol.

$0.97 \mathrm{~g}$ (50\% yield for two steps), solid, mp. $42.1-43.5^{\circ} \mathrm{C}$. 
${ }^{1} \mathbf{H}$ NMR (600 MHz, CDCl 3$) \delta 7.29(\mathrm{~d}, J=12.0 \mathrm{~Hz}, 2 \mathrm{H}), 7.20(\mathrm{~d}, J=12.0 \mathrm{~Hz}, 2 \mathrm{H})$, 4.65 (s, 2 H), 2.72-2.68 (m, 2 H), 1.80-1.76 (m, 2 H), 1.53 (s, 2 H), 1.29 (s, $6 \mathrm{H})$.

${ }^{13}$ C NMR (150 MHz, CDCl 3$) \delta 142.1,138.4,128.5,127.2,70.9,65.2,45.7,30.4,29.3$.

IR (neat, $\mathbf{c m}^{-1}$ ): 3325, 2974, 2932, 2866, 2361, 1620, 1515, 1457, 1420, 1381, 1211, 1150, 1016, 926, 909, 848, 816.

HRMS (ESI): $[\mathrm{M}+\mathrm{Na}]^{+}$calcd for $\mathrm{C}_{12} \mathrm{H}_{18} \mathrm{NaO}_{2} 217.1199$, found 217.1197.

(3R,5R,6S,8R,9S,10R,12S,13R,14S,17R)-17-((R)-5-hydroxy-5-methylhexan-2-yl)10,13-dimethylhexadecahydro-1H-cyclopenta[a]phenanthrene-3,6,12-triol (1af)

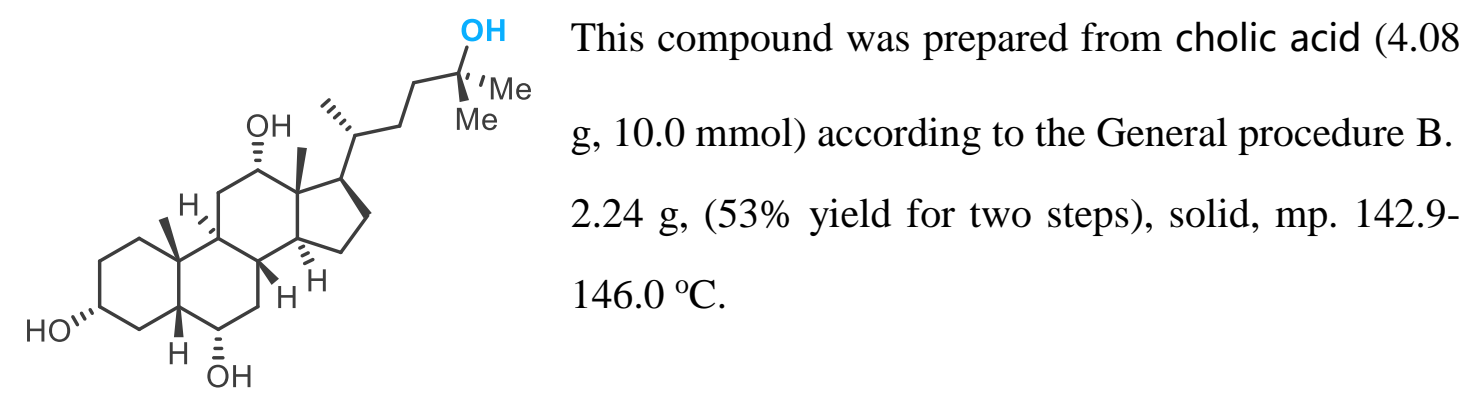

${ }^{1}$ H NMR (600 MHz, CDCl$)$ ) $83.65-3.59$ (m, $\left.1 \mathrm{H}\right)$, 1.98-1.25 (m, $\left.24 \mathrm{H}\right), 1.20-1.00(\mathrm{~m}$, $12 \mathrm{H}), 0.92-0.91(\mathrm{~m}, 6 \mathrm{H}), 0.64(\mathrm{~s}, 3 \mathrm{H})$.

${ }^{13}$ C NMR (150 MHz, CDCl $) \delta$ 71.8, 71.2, 56.5, 55.9, 42.7, 42.1, 40.4, 40.2, 40.0, 36.4, $35.9,35.8,35.3,34.6,30.5,30.1,29.3,29.0,28.2$, 27.2, 26.4, 24.2, 23.4, 20.8, 18.7, 12.0.

IR (neat, $\mathbf{c m}^{-1}$ ): 2391, 2865, 1467, 1448, 1377, 1217, 1156, 1086, 1072, 1047, 960, 945, 913, 800, 738.

HRMS (ESI): $[\mathrm{M}+\mathrm{Na}]^{+}$calcd for $\mathrm{C}_{26} \mathrm{H}_{46} \mathrm{NaO}_{4} 445.3288$, found 445.3283 . 


\subsection{Preparation of Vinyl Halides (ratios of E:Z are provided)}<smiles>Br/C=C/c1ccccc1</smiles>

2a (>20:1)<smiles>Fc1ccc(/C=C/Cl)cc1</smiles>

$2 f(>20: 1)$<smiles>CC(=O)c1cccc(/C=C/Br)c1</smiles>

2k (9:1)<smiles>Br/C=C\c1ccccc1</smiles>

2b (<1: 20)<smiles>I/C=C/c1ccccc1</smiles>

$\mathbf{2 g}(17: 1)$<smiles>COC(=O)c1ccccc1/C=C/Br</smiles>

2I $(11: 1)$<smiles>Br/C=C/c1ccco1</smiles>

$\mathbf{2 q}(2: 1)$

$2 \mathbf{v}(>20: 1)$<smiles>CC(/C=C/c1ccccc1)=C\Br</smiles><smiles>F/C=C/c1ccccc1</smiles>

2c $(1: 3.4)$<smiles>Cc1cccc(C)c1/C=C/Br</smiles>

2h (9:1)<smiles>FC(F)(F)c1ccc(/C=C/Br)cc1</smiles>

$2 \mathrm{~m}(>20: 1)$

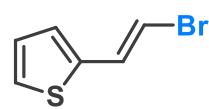

$2 \mathbf{r}(2.5: 1)$<smiles>C/C=C/c1ccc(C)cc1</smiles>

2d (1:4)

${ }^{\mathrm{t}} \mathrm{Bu}$<smiles>CC(C)c1cc(I)cc(/C=C/Br)c1</smiles>

2i (18: 1)<smiles>Fc1ccc(/C=C/Br)cc1F</smiles>

2n (13: 1)<smiles>C/C(=C\Br)c1ccccc1</smiles><smiles>Br/C=C/C=C/c1ccccc1</smiles>

2s $(>20: 1)$

2t $(4.5: 1)$<smiles>Cl/C=C/c1ccccc1</smiles>

$2 e(>20: 1)$<smiles>CSc1ccc(/C=C/Br)cc1</smiles>

2j (0.9: 1)<smiles>N#Cc1ccc(/C=C/Br)cc1</smiles>

$20(9: 1)$ 2u $(2.9: 1)$<smiles>Clc1ccc(/C=C/Br)cc1</smiles>

$\mathbf{2 z}(8: 1)$<smiles>Br/C=C/c1ccc(Br)cc1</smiles>

2aa $(8: 1)$<smiles>CC(C)=CC=CBr</smiles>

$2 \times(1.6: 1)$

$$
\text { 2w (1:1) }
$$<smiles>Br/C=C/c1ccc(I)cc1</smiles>

2ab (10:1)

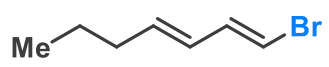

$2 y(1.5: 1)$<smiles>Br/C=C/c1ccc(Oc2ccccc2)cc1</smiles>

2ad (11: 1)<smiles>Oc1ccc(/C=C/Br)cc1</smiles>

2ae $(7: 1)$<smiles>Br/C=C/C1CC1c1ccccc1</smiles><smiles>Br/C=C(/CBr)c1ccccc1</smiles><smiles>C=C(CO[SnH3])c1ccccc1</smiles>

Compounds $2 \mathrm{a}$ is commercially available. Known compound $2 \mathrm{~b},{ }^{3} \mathbf{2 c},{ }^{4} \mathbf{2 d},{ }^{4} \mathbf{2 e},{ }^{\mathbf{5}} \mathbf{2 f},{ }^{\mathbf{5}}$ $2 \mathrm{~g},{ }^{6} 2 \mathrm{~m},{ }^{7} 2 \mathrm{n},{ }^{8} 2 \mathrm{o},{ }^{9} 2 \mathrm{p},{ }^{10} 2 \mathrm{q},{ }^{11} 2 \mathrm{r},{ }^{5} 2 \mathrm{~s},{ }^{12} 2 \mathrm{t},{ }^{13} 2 \mathrm{v},{ }^{14} 2 \mathrm{x},{ }^{15} 2 \mathrm{y},{ }^{16} 2 \mathrm{z},{ }^{7} 2 \mathrm{aa},{ }^{16} 2 \mathrm{ab},{ }^{17}$ and $12^{18}$ were prepared according to the literature procedure. The preparation of new compounds, and their characterization data are provided below. 


\section{General Procedure C}

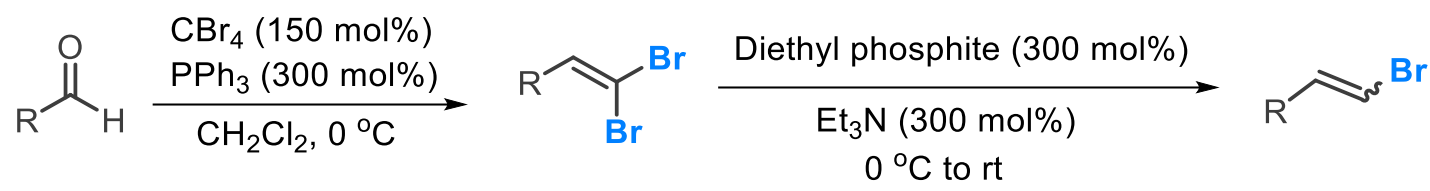

Step 1: To a flame-dried flask was added aldehyde (10.0 mmol), $\mathrm{CBr}_{4}$ (4.98 g, 15.0 mmol), and $\mathrm{CH}_{2} \mathrm{Cl}_{2}(30 \mathrm{~mL})$. The flask was cooled to $0{ }^{\circ} \mathrm{C}$, at which point a solution of $\mathrm{PPh}_{3}(7.87 \mathrm{~g}, 30 \mathrm{mmol})$ in $\mathrm{CH}_{2} \mathrm{Cl}_{2}(20 \mathrm{~mL})$ was added dropwise via addition funnel over $30 \mathrm{~min}$. The reaction mixture was stirred at $0{ }^{\circ} \mathrm{C}$ under $\mathrm{N}_{2}$ for $1 \mathrm{~h}$. After removing half of the volume of $\mathrm{CH}_{2} \mathrm{Cl}_{2}$ under reduced pressure, pentane $(50 \mathrm{~mL})$ was added to precipitate triphenylphosphine oxide (TPPO). The mixture was filtered and the filtrate was concentrated in vacuum. The residue was dissolved in pentane $(30 \mathrm{~mL})$, which led to further precipitation of TPPO. The mixture was filtered and the filtrate was concentrated to afford dibromide which was used directly for the next step.

Step 2: To a solution of the crude dibromide ( 10.0 mmol) and $\mathrm{Et}_{3} \mathrm{~N}(3.04 \mathrm{~g}, 30 \mathrm{mmol})$ in DMF $(15 \mathrm{~mL})$ was added dimethylphosphite $(3.30 \mathrm{~g}, 30.0 \mathrm{mmol})$. The reaction mixture was stirred at room temperature for over night. The reaction was quenched with water $(30 \mathrm{~mL})$ and the mixture was extracted with pentane $(2 \times 30 \mathrm{~mL})$. The combined organic layers were washed with an aqueous solution of $\mathrm{HCl}(1 \mathrm{M}, 30 \mathrm{~mL})$, dried over anhydrous $\mathrm{Na}_{2} \mathrm{SO}_{4}$, filtered, and concentrated. The residue was purified by flash chromatography to afford the desired product.

\section{2-(2-Bromovinyl)-1,3-dimethylbenzene (2h)}<smiles>Cc1cccc(C)c1/C=C/Br</smiles>

This compound was prepared from 2,6-dimethylbenzaldehyde (1.34 $\mathrm{g}, 10.0 \mathrm{mmol}$ ) according to the General procedure C.

$1.34 \mathrm{~g}(64 \%$ yield for 2 steps, $\mathrm{E} / \mathrm{Z}=9$ : 1), colorless oil.

${ }^{1} \mathrm{H}$ NMR (400 MHz, CDCl 3$) \delta[7.49(\mathrm{~d}, J=8.4 \mathrm{~Hz}), 7.27(\mathrm{~d}, J=13.6 \mathrm{~Hz}), 1 \mathrm{H}], 7.20$ $(\mathrm{d}, J=8.4 \mathrm{~Hz}, 1 \mathrm{H}), 6.97(\mathrm{~s}, 2 \mathrm{H}),[6.58(\mathrm{~d}, J=14.0 \mathrm{~Hz}, 0.9 \mathrm{H}), 6.47(\mathrm{~d}, J=7.6 \mathrm{~Hz}, 0.1$ H)], [2.32(s), 2.29 (s), $6 \mathrm{H}]$.

${ }^{13}$ C NMR (100 MHz, CDCl $) \delta 138.1,135.3,135.0,132.3,131.2,127.0,125.7,106.2$, 21.1, 19.7 
IR (neat, $\mathbf{c m}^{-1}$ ): 3071, 3005, 2970, 2946, 1613, 1599, 1562, 1496, 1261, 1192, 935, $780,705$.

HRMS (ESI): $[\mathrm{M}+\mathrm{H}]^{+}$calcd for $\mathrm{C}_{10} \mathrm{H}_{12} \mathrm{Br} 211.0117$, found 211.0117.

\section{1-(2-Bromovinyl)-3,5-di-tert-butylbenzene (2i)}

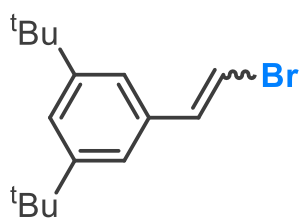

This compound was prepared from 3,5-di-tert-butylbenzaldehyde

$(2.18 \mathrm{~g}, 20.0 \mathrm{mmol})$ according to the General procedure $\mathrm{C}$.

$1.77 \mathrm{~g}(60 \%$ yield for 2 steps, $\mathrm{E} / \mathrm{Z}=18: 1)$, colorless oil.

${ }^{1}$ H NMR (400 MHz, CDCl3) $\delta[7.56(\mathrm{~s}), 7.37(\mathrm{~s}), 1 \mathrm{H}]$, 7.15-7.12 (m,

$3 \mathrm{H}),[6.74(\mathrm{~d}, J=14.0 \mathrm{~Hz}, 0.9 \mathrm{H}), 6.39$ (d, $J=8.0 \mathrm{~Hz}, 0.05 \mathrm{H})], 1.32(\mathrm{~s}, 18 \mathrm{H})$.

${ }^{13}$ C NMR (100 MHz, CDCl3) $\delta$ 151.2, 138.1, 135.2, 122.6, 120.4, 105.7, 34.8, 31.4.

IR (neat, cm$^{-1}$ ): 2964, 2903, 2868, 1591, 1477, 1394, 1363, 1249, 1215, 938, 913, 746.

HRMS (ESI): $[\mathrm{M}+\mathrm{H}]^{+}$calcd for $\mathrm{C}_{16} \mathrm{H}_{24} \mathrm{Br} 295.1056$, found 295.1040.

(4-(2-Bromovinyl)phenyl)(methyl)sulfane (2j)

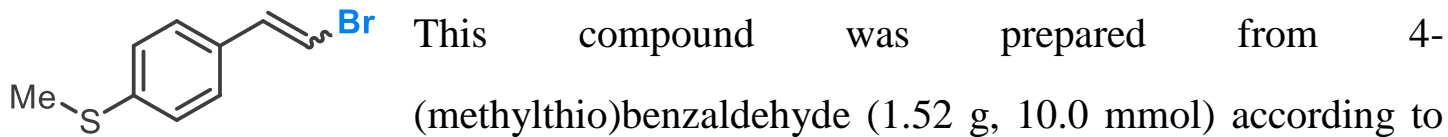
the General procedure $\mathrm{C}$.

$1.41 \mathrm{~g},(62 \%$ yield for 2 steps, $\mathrm{E} / \mathrm{Z}=0.9: 1)$, colorless oil.

${ }^{1}$ H NMR (400 MHz, CDCl3) $\delta 7.63(\mathrm{~d}, J=8.4$ Hz, $1 \mathrm{H})$, 7.26-7.17 (m, 3 H), [7.04 (d, $J=14.0 \mathrm{~Hz}), 7.00(\mathrm{~d}, J=8.4 \mathrm{~Hz}), 1 \mathrm{H}],[6.72(\mathrm{~d}, J=14.0 \mathrm{~Hz}, 0.5 \mathrm{H}), 6.38(\mathrm{~d}, J=8.4 \mathrm{~Hz}$, $0.54 \mathrm{H})],[2.50(\mathrm{~s}), 2.48(\mathrm{~s}), 3 \mathrm{H}]$.

${ }^{13}$ C NMR (100 MHz, CDCl3) $\delta$ 139.0, 138.9, 136.5, 132.6, 131.6, 131.5, 129.3, 126.4, $125.7,105.8,105.7,15.5,15.4$.

IR (neat, $\mathbf{c m}^{-1}$ ): 3072, 3020, 2985, 2954, 2920, 1655, 1593, 1436, 1401, 1120, 1092, $931,722,752,726$.

HRMS (ESI): $[\mathrm{M}+\mathrm{H}]^{+}$calcd for $\mathrm{C}_{9} \mathrm{H}_{10} \mathrm{BrS} 228.9681$, found 228.9677 .

\section{Methyl 3-(2-bromovinyl)benzoate (2k)}

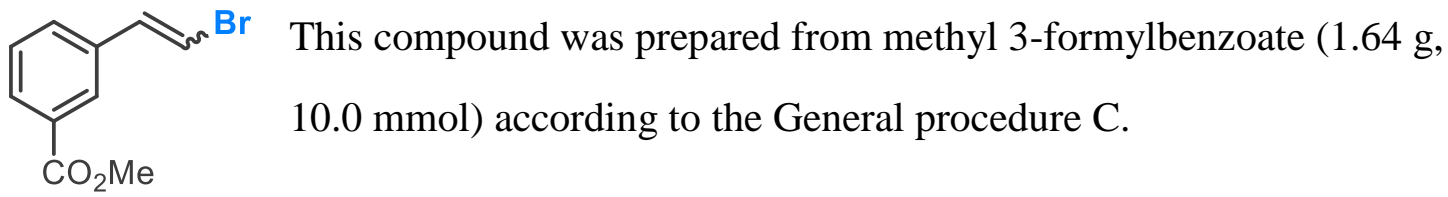


$1.15 \mathrm{~g}$ (48\% yield for 2 steps, $\mathrm{E} / \mathrm{Z}=9: 1)$, colorless oil.

${ }^{1} \mathbf{H}$ NMR (600 MHz, CDCl $) \delta 7.99(\mathrm{~s}, 1 \mathrm{H}),[7.96(\mathrm{~d}, J=7.8 \mathrm{~Hz}), 7.92(\mathrm{~d}, J=7.8 \mathrm{~Hz})$, $1 \mathrm{H}], 7.48(\mathrm{~d}, J=7.8 \mathrm{~Hz}, 1 \mathrm{H}), 7.41(\mathrm{t}, J=7.8 \mathrm{~Hz}, 1 \mathrm{H}), 7.14(\mathrm{~d}, J=13.8 \mathrm{~Hz}, 1 \mathrm{H}),[6.88$ (d, $J=14.4 \mathrm{~Hz}, 0.9 \mathrm{H}), 6.52(\mathrm{~d}, J=8.4 \mathrm{~Hz}, 0.1 \mathrm{H})],[3.94$ (s), 3.93 (s), $3 \mathrm{H}]$.

${ }^{13}$ C NMR (150 MHz, CDCl 3$) \delta 166.6,136.2,130.7,130.3,130.2,129.2,128.9,127.1$, 108.0, 52.3.

IR (neat, $\mathbf{c m}^{-1}$ ): 3075, 3020, 2951, 1724, 1606, 1582, 1483, 1292, 1218, 1172, 1109, $895,743$.

HRMS (ESI): $[\mathrm{M}+\mathrm{Na}]^{+}$calcd for $\mathrm{C}_{10} \mathrm{H}_{9} \mathrm{BrNaO}_{2} 262.9678$, found 262.9680 .

Methyl 2-(2-bromovinyl)benzoate (2l)

Br This compound was prepared from methyl 2-formylbenzoate (1.64 g, $\mathrm{CO}_{2} \mathrm{Me} \quad 10.0 \mathrm{mmol}$ ) according to the General procedure C.

$1.25 \mathrm{~g}(52 \%$ yield for 2 steps, $\mathrm{E} / \mathrm{Z}=11: 1)$, colorless oil.

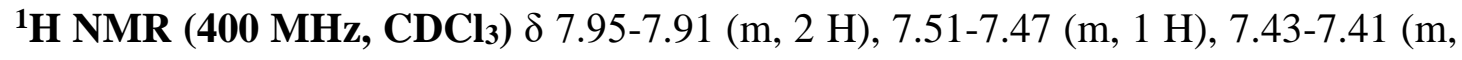
$1 \mathrm{H}), 7.39-7.35(\mathrm{~m}, 1 \mathrm{H}),[6.67(\mathrm{~d}, J=14.0 \mathrm{~Hz}, 1 \mathrm{H}), 6.52(\mathrm{~d}, J=8.0 \mathrm{~Hz}, 0.09 \mathrm{H})]$, [3.91 (s), 3.89 (s), $3 \mathrm{H}]$.

${ }^{13}$ C NMR (100 MHz, CDCl 3$) \delta 167.2,137.5,136.4,132.4,130.8,128.0,127.8,127.5$, 108.4, 52.2.

IR (neat, $\mathbf{c m}^{-1}$ ): 3076, 3951, 1720, 1603, 1565, 1434, 1260, 1166, 1131, 1078, 1049 , 936, 758, 732 .

HRMS (ESI): $[\mathrm{M}+\mathrm{Na}]^{+}$calcd for $\mathrm{C}_{10} \mathrm{H}_{9} \mathrm{BrNaO}_{2} 262.9678$, found 262.9669.

((1E)-4-bromo-3-methylbuta-1,3-dien-1-yl)benzene (2u)<smiles>CC(C=Cc1ccccc1)=CBr</smiles>

This compound was prepared from (E)-4-phenylbut-3-en-2-one (1.46 g, $10.0 \mathrm{mmol})$ according to the General procedure C.

$1.27 \mathrm{~g}(57 \%$ yield for 2 steps, $\mathrm{E} / \mathrm{Z}=2.9: 1)$, colorless oil.

${ }^{1} \mathbf{H}$ NMR (600 MHz, CDCl$) ~ \delta[7.50(\mathrm{~d}, J=7.8 \mathrm{~Hz}), 7.42(\mathrm{~d}, J=7.8 \mathrm{~Hz}), 2 \mathrm{H}], 7.35$ 7.30 (m, 2 H), 7.26-7.25 (m, 1 H), 6.79-6.62 (m, 2 H), [6.41 (s), 6.14 (s), 1H], [2.05 (s, $2.16 \mathrm{H}), 2.01(\mathrm{~s}, 0.73 \mathrm{H})]$.

${ }^{13}$ C NMR (150 MHz, CDCl3) $\delta$ 139.8, 136.9, 136.7, 132.1, 129.0, 128.8, 128.7, 128.1, 
$127.7,126.8,126.4,126.1,110.0,105.9,19.5,15.4$.

IR (neat, cm$^{-1}$ ): 3087, 3029, 2982, 1566, 1493, 1360, 1292, 928, 727, 665.

HRMS (ESI): $[\mathrm{M}+\mathrm{H}]^{+}$calcd for $\mathrm{C}_{11} \mathrm{H}_{12} \mathrm{Br} 223.0117$, found 223.0114.

\section{((2E)-5-bromopenta-2,4-dien-2-yl)benzene (2w)}<smiles>CC(=CC=CBr)c1ccccc1</smiles>

This compound was prepared from (E)-3-phenylbut-2-enal (1.46 $\mathrm{g}, 10.0 \mathrm{mmol}$ ) according to the General procedure C.

$1.18 \mathrm{~g}(53 \%$ yield for 2 steps, $\mathrm{E} / \mathrm{Z}=1: 1)$.

${ }^{1} \mathrm{H}$ NMR $\left(600 \mathrm{MHz}, \mathbf{C D C l}_{3}\right) \delta 7.50(\mathrm{~d}, J=11.0 \mathrm{~Hz}, 1 \mathrm{H}), 7.43(\mathrm{~d}, J=11.0 \mathrm{~Hz}, 1 \mathrm{H})$, 7.37-7.24 (m, $3 \mathrm{H}), 7.12\left(\mathrm{dd}, J_{l}=16.8 \mathrm{~Hz}, J_{2}=19.8 \mathrm{~Hz}, 0.5 \mathrm{H}\right), 7.01\left(\mathrm{dd}, J_{l}=10.8 \mathrm{~Hz}\right.$, $\left.J_{2}=15.6 \mathrm{~Hz}, 0.5 \mathrm{H}\right), 6.76(\mathrm{~d}, J=15.6 \mathrm{~Hz}, 0.5 \mathrm{H}), 6.40(\mathrm{~d}, J=19.8 \mathrm{~Hz}, 0.5 \mathrm{H}), 6.35(\mathrm{~d}$, $J=16.8 \mathrm{~Hz}, 0.5 \mathrm{H}), 6.29$ (d, $J=10.8 \mathrm{~Hz}, 0.5 \mathrm{H}), 2.19$ (s, 1.5H), $2.14(\mathrm{~s}, 1.5 \mathrm{H})$.

${ }^{13}$ C NMR (150 MHz, CDCl3) $\delta$ 142.5, 142.4, 140.6, 137.5, 134.5, 129.0, 128.4, 127.7, $127.5,125.9,125.6,124.1,122.5,109.3,109.2,16.9,16.2$.

IR (neat, cm-1): 3081, 3061, 3028, 2989, 2946, 2860, 1563, 1493, 1444, 1355, 938, 789, 697, 666 .

HRMS (ESI): $[\mathrm{M}+\mathrm{H}]^{+}$calcd for $\mathrm{C}_{11} \mathrm{H}_{12} \mathrm{Br} 223.0117$, found 223.0114.

\section{4-(2-Bromovinyl)phenyl methanesulfonate (2ac)}

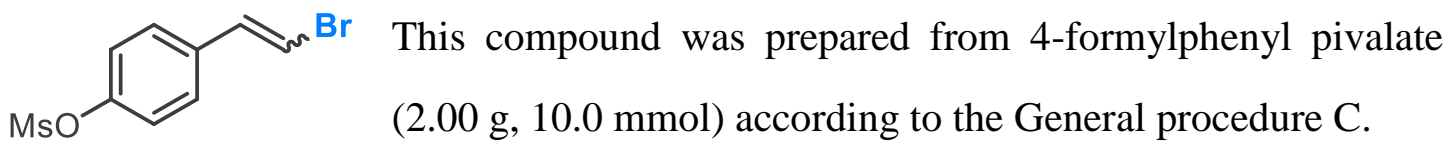
$1.32 \mathrm{~g} \mathrm{(48 \%} \mathrm{yield} \mathrm{for} 2$ steps, $\mathrm{E} / \mathrm{Z}=10: 1)$, colorless oil.

${ }^{1} \mathbf{H}$ NMR (400 MHz, CDCl 3$) \delta 7.35(\mathrm{~d}, J=8.8 \mathrm{~Hz}, 2 \mathrm{H}), 7.25(\mathrm{~d}, J=8.8 \mathrm{~Hz}, 2 \mathrm{H})$, $[7.10(\mathrm{~d}, J=14.0 \mathrm{~Hz}, 0.9 \mathrm{H}), 7.06(\mathrm{~d}, J=8.4 \mathrm{~Hz}, 0.09 \mathrm{H})],[6.79(\mathrm{~d}, J=14.0 \mathrm{~Hz}), 6.50$ $(\mathrm{d}, J=8.4 \mathrm{~Hz}), 1 \mathrm{H}], 3.15(\mathrm{~s}, 3 \mathrm{H})$.

${ }^{13}$ C NMR (100 MHz, CDCl 3$) \delta 148.8,135.7,135.2,127.6,122.4,107.9,37.5$.

IR (neat, $\left.\mathbf{c m}^{-1}\right)$ : 2074, 1498, 1638, 1413, 1362, 1142, 1018, 682.

HRMS (ESI): $[\mathrm{M}+\mathrm{Na}]^{+}$calcd for $\mathrm{C}_{9} \mathrm{H}_{9} \mathrm{BrNaO}_{3} \mathrm{~S} 298.9348$, found 298.9348 . 


\section{4-(2-Bromovinyl)phenyl pivalate (2ad)}

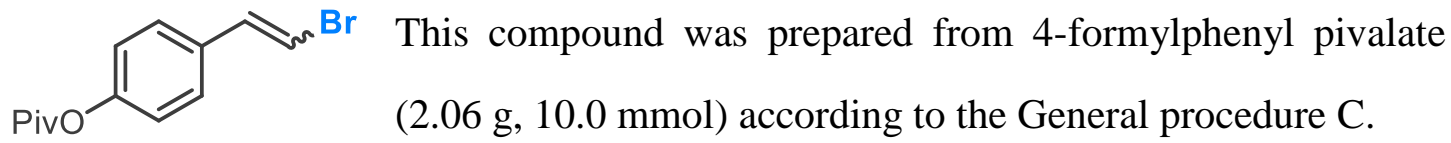

$1.69 \mathrm{~g}(60 \%$ yield for 2 steps, $\mathrm{E} / \mathrm{Z}=11: 1)$, colorless oil.

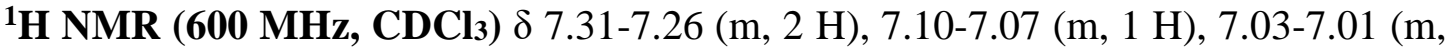
$2 \mathrm{H}),[6.73(\mathrm{~d}, J=21 \mathrm{~Hz}), 6.42(\mathrm{~d}, J=12.6 \mathrm{~Hz}), 1 \mathrm{H}],[1.36(\mathrm{~s}, 0.73 \mathrm{H}), 1.35(\mathrm{~s}, 8.3$ $\mathrm{H})]$.

${ }^{13}$ C NMR (150 MHz, CDCl 3$) \delta 176.9,151.0,136.3,133.4,127.0,121.9,106.5,39.1$, 27.1.

IR (neat, $\mathbf{c m}^{-1}$ ): 3079, 2978, 2939, 2875, 1750, 1601, 1476, 1459, 1366, 1282, 1205, $1169,1132,904,846,773,732$.

HRMS (ESI): $[\mathrm{M}+\mathrm{Na}]^{+}$calcd for $\mathrm{C}_{13} \mathrm{H}_{15} \mathrm{BrNaO}_{2} 305.0148$, found 305.0145 .

\section{4-(2-Bromovinyl)phenyl trifluoromethanesulfonate (2ae)}

Br This compound was prepared from 4-formylphenyl
trifluoromethanesulfonate $(2.54 \mathrm{~g}, 10.0 \mathrm{mmol})$ according to the

General procedure C.

$2.44 \mathrm{~g}$ (74\% yield for 2 steps, $\mathrm{E} / \mathrm{Z}=7: 1)$, colorless oil.

${ }^{1} \mathbf{H}$ NMR $\left(600 \mathrm{MHz}, \mathbf{C D C l}_{3}\right) \delta[7.76(\mathrm{~d}, J=8.4 \mathrm{~Hz}), 7.37(\mathrm{~d}, J=8.4 \mathrm{~Hz}), 2 \mathrm{H}], 7.29$ $7.23(\mathrm{~m}, 2 \mathrm{H}), 7.11-7.06(\mathrm{~m}, 1 \mathrm{H}),[6.83(\mathrm{~d}, J=13.8 \mathrm{~Hz}, 0.88 \mathrm{H}), 6.54(\mathrm{~d}, J=8.4 \mathrm{~Hz}$, $0.12 \mathrm{H})]$.

${ }^{13}$ C NMR (150 MHz, CDCl 3 ) $\delta 149.0,136.3,135.3,127.7,121.8,118.7$ (q, $J=318.0$ $\mathrm{Hz}), 108.8$.

${ }^{19}$ F NMR (376 MHz, CDCl 3$): ~ \delta-72.8$.

IR (neat, $\mathbf{c m}^{-1}$ ): 3077, 1614, 1586, 1502, 1425, 1250, 1215, 1179, 1140, 1017, 950, 718.

HRMS (ESI): $[\mathrm{M}+\mathrm{H}]^{+}$calcd for $\mathrm{C}_{9} \mathrm{H}_{7} \mathrm{BrF}_{3} \mathrm{O}_{3} \mathrm{~S} 330.9246$, found 330.9243 . 


\section{(2-(2-Bromovinyl)cyclopropyl)benzene (4)}

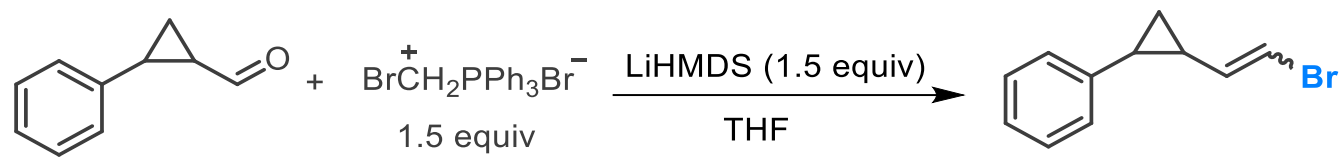

To a stirred solution of $\left[\mathrm{Ph}_{3} \mathrm{P}^{+} \mathrm{CH}_{2} \mathrm{Br}\right] \mathrm{Br}^{-}(3.27 \mathrm{~g}, 7.5 \mathrm{mmol})$ in dry THF $(25 \mathrm{~mL})$ was dropwise added LiHMDS (7.5 mL, 7.5mmol, $1.0 \mathrm{M}$ in THF) at $0{ }^{\circ} \mathrm{C}$ under nitrogen atmosphere. The reaction mixture was stirred for another $30 \mathrm{~min}$ at the same temperature. A solution of 2-phenylcyclopropane-1-carbaldehyde (730 mg, $5.0 \mathrm{mmol}$ ) in dry THF ( $8 \mathrm{~mL})$ was added dropwise, and the resulting solution was stirred at room temperature overnight. The reaction was quenched with water $(30 \mathrm{~mL})$, and the mixture was extracted with EtOAc $(30 \mathrm{~mL} \times 3)$. The combined organic layers were washed with brine $(20 \mathrm{~mL})$, dried over anhydrous $\mathrm{Na}_{2} \mathrm{SO}_{4}$, filtered, and concentrated in vacuum. The residue was purified by flash chromatography on silica gel to give (2-(2bromovinyl)cyclopropyl)benzene.

$0.72 \mathrm{~g}(65 \%$ yield, $\mathrm{E} / \mathrm{Z}=1: 4)$, colorless oil.

${ }^{1}$ H NMR (400 MHz, CDCl 3$) \delta 7.28(\mathrm{t}, J=7.2 \mathrm{~Hz}, 2 \mathrm{H}), 7.19(\mathrm{~d}, J=7.2 \mathrm{~Hz}, 1 \mathrm{H}), 7.16-$ $7.05(\mathrm{~m}, 2 \mathrm{H}),[6.13(\mathrm{~d}, J=7.2 \mathrm{~Hz}), 6.09(\mathrm{~d}, J=13.6 \mathrm{~Hz}), 1 \mathrm{H}],\left[5.88\left(\mathrm{dd}, J_{l}=8.8 \mathrm{~Hz}\right.\right.$, $\left.\left.J_{2}=13.2 \mathrm{~Hz}, 0.2 \mathrm{H}\right), 5.68\left(\mathrm{dd}, J_{1}=7.2 \mathrm{~Hz}, J_{2}=9.2 \mathrm{~Hz}, 0.8 \mathrm{H}\right)\right], 2.17-1.63(\mathrm{~m}, 2 \mathrm{H})$, 1.35-1.30 (m, $1 \mathrm{H}), 1.17-1.12(\mathrm{~m}, 1 \mathrm{H})$.

${ }^{13}$ C NMR (100 MHz, CDCl3) $\delta 141.3,137.1,128.4,126.1,126.0,105.7,25.0,24.3$, 16.7 .

IR (neat, $\mathbf{c m}^{-1}$ ): 3088, 3082, 1625, 1459, 1388, 1288, 1273, 1058, 937, 900, 837, 751, 697.

HRMS (ESI): $[\mathrm{M}+\mathrm{H}]^{+}$calcd for $\mathrm{C}_{11} \mathrm{H}_{12} \mathrm{Br} 223.0117$, found 223.0116 .

\section{(Z)-(3-(benzyloxy)-1-bromoprop-1-en-2-yl)benzene (8)}

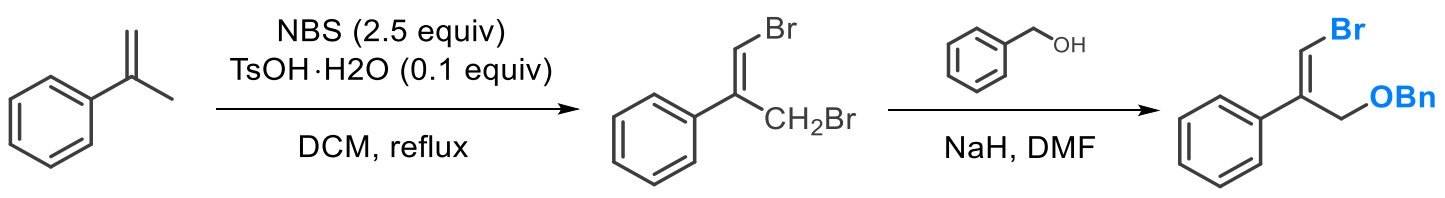

Step 1: To a solution of prop-1-en-2-ylbenzene (1 g, $8.5 \mathrm{mmol}, 1.0$ equiv) and 
$\mathrm{TsOH} \cdot \mathrm{H}_{2} \mathrm{O}(155 \mathrm{mg}, 0.85 \mathrm{mmol}, 0.1$ equiv) in DCM (30 mL) was added NBS (3.8 g, $21.2 \mathrm{mmol}, 2.5$ equiv). After refluxing for overnight, water (30 mL) and DCM (30 mL) were added at room temperature and separated. The water phase was extracted with DCM $(20 \mathrm{~mL} \times 2)$. The combined organic phases were washed with brine $(30 \mathrm{~mL})$, dried over anhydrous $\mathrm{Na}_{2} \mathrm{SO}_{4}$, and concentrated under reduced pressure. The residue was purified by flash chromatography on silica gel to give (Z)-(1,3-dibromoprop-1-en2-yl)benzene as a colorless oil (1.65 g, 71\% yield).

Step 2: To a solution of $\mathrm{NaH}(60 \%, 135 \mathrm{mg}, 3.36 \mathrm{mmol}, 1.5$ equiv) in $\mathrm{DMF}(8 \mathrm{~mL})$ was dropwise added a solution of phenylmethanol (242 mg, $2.24 \mathrm{mmol}, 1.0$ equiv) in DMF (4 mL) at $0{ }^{\circ} \mathrm{C}$ under $\mathrm{N}_{2}$ atmosphere. After stirring at $0{ }^{\circ} \mathrm{C}$ for $1.5 \mathrm{~h},(\mathrm{Z})-(1,3-$ dibromoprop-1-en-2-yl)benzene (0.86 g, $3.14 \mathrm{mmol}, 1.4$ equiv) was added. The reaction mixture was allowed to stir at room temperature for overnight. EtOAc $(20 \mathrm{~mL})$ and water $(20 \mathrm{~mL})$ were added. The mixture solution was separated and the aqueous phase was extracted with EtOAc $(20 \mathrm{~mL} \times 2)$. The combined organic phases were washed with brine $(20 \mathrm{~mL})$, dried over anhydrous $\mathrm{Na}_{2} \mathrm{SO}_{4}$, and concentrated in vacuum. The residue was purified by flash chromatography on silica gel to give (Z)-(1,3dibromoprop-1-en-2-yl)benzene as a colorless oil (0.46 g, 68\% yield).

${ }^{1}$ H NMR (400 MHz, CDCl $)$ ) $7.42-7.39$ (m, 2 H), 7.37-7.25 (m, 8 H), $6.64(\mathrm{~s}, 1 \mathrm{H})$, $4.63(\mathrm{~s}, 2 \mathrm{H}), 4.52$ (s, $2 \mathrm{H})$.

${ }^{13}$ C NMR (100 MHz, CDCl 3$) \delta 142.3,138.6,137.9,128.5,128.3,128.1,127.9,127.7$, $126.7,109.0,72.2,69.0$.

IR (neat, $\mathbf{c m}^{-1}$ ): 2082, 1634, 1495, 1454, 1359, 1312, 1243, 1217, 1075, 942, 904, 808, $734,695$.

HRMS (ESI): $[\mathrm{M}+\mathrm{Na}]^{+}$calcd for $\mathrm{C}_{16} \mathrm{H}_{15} \mathrm{BrNaO} 325.0198$, found 325.0916 . 


\section{Ti-Catalyzed Stereoconvergent Radical Dehydroxylative Vinylation of Tertiary Alcohols with Vinyl Halides}

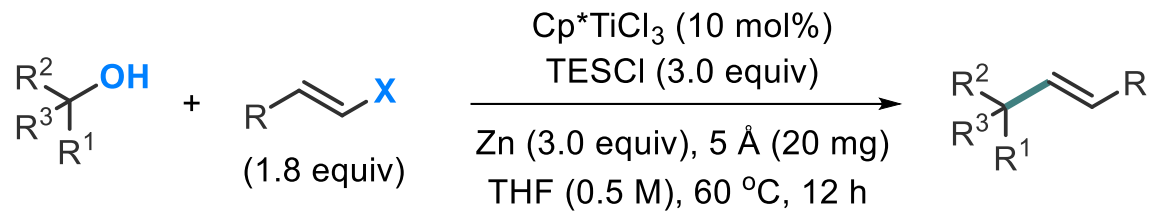

General Procedure D: The procedure was conducted in an argon-filled glove box. To a reaction tube equipped with a magnetic stir bar was charged with $\mathrm{Cp}^{*} \mathrm{TiCl}_{3}(5.90 \mathrm{mg}$, $0.02 \mathrm{mmol}), 5 \AA \mathrm{MS}(20 \mathrm{mg}), \mathrm{Zn}$ (39 mg, $0.6 \mathrm{mmol})$, alcohols 1 (0.2 mmol), vinyl halides 2 (0.36 mmol), THF (0.4 mL), and TESCl (90.6 mg, $0.6 \mathrm{mmol})$. The reaction tube was sealed and removed from the glove box. The reaction mixture was stirred at $60{ }^{\circ} \mathrm{C}$ for $12 \mathrm{~h}$. The solvent was removed under the reduced pressure. The residue was purified by flash chromatography on silica gel to afford the desired product.

\section{Characterization Data of Products}

\section{(E)-(3,3-dimethylpent-1-ene-1,5-diyl)dibenzene (3a, known compound)}

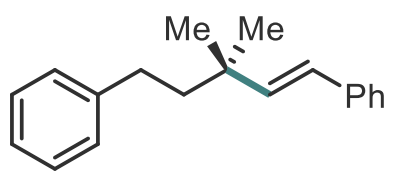

The title compound was prepared according to the General

Procedure D from the reaction of 2-methyl-4-phenylbutan-2ol 1a (32.8 mg, $0.20 \mathrm{mmol})$ with (E)-(2-bromovinyl)benzene 2a (65.5 mg, $0.36 \mathrm{mmol}) .43 .0 \mathrm{mg}, 86 \%$ yield, colorless oil. ${ }^{1} \mathrm{H} \mathrm{NMR}$ and ${ }^{13} \mathrm{C}$ NMR data are consistent with those reported in reference 7.

Gram-scale reaction: The target compound $(0.9 \mathrm{~g}, 73 \%$ yield $)$ was prepared according to the General Procedure D. $\mathrm{Cp}^{*} \mathrm{TiCl}_{3}$ (147.5 mg, $\left.0.5 \mathrm{mmol}\right), 5 \AA \mathrm{MS}(0.5 \mathrm{~g}), \mathrm{Zn}(0.98$ g, 15 mmol), 2-methyl-4-phenylbutan-2-ol $1 \mathbf{a}(0.82$ g, 5 mmol), (E)-(2bromovinyl)benzene 2a (1.64 g, 9 mmol), TESCl (2.27 g, $15 \mathrm{mmol})$, and THF (10 mL) were used.

Reaction of 1a with bromide $2 \mathbf{b}$ : The title compound was prepared according to the General Procedure D. The reaction of $1 \mathbf{a}(32.8 \mathrm{mg}, 0.20 \mathrm{mmol})$ and $(\mathrm{Z})-(2-$ 
bromovinyl)benzene $\mathbf{2 b}(65.5 \mathrm{mg}, 0.36 \mathrm{mmol})$ afforded $\mathbf{3 a}$ in $51 \%$ yield $(25.5 \mathrm{mg})$.

Reaction of 1a with fluoride 2c: The title compound was prepared according to the General Procedure D. The reaction of $1 \mathrm{a}(32.8 \mathrm{mg}, 0.20 \mathrm{mmol})$ and (2fluorovinyl)benzene $2 \mathbf{c}(43.9 \mathrm{mg}, 0.36 \mathrm{mmol}, \mathrm{E}: \mathrm{Z}=1: 3.4)$ afforded 3a in $60 \%$ yield (30.0 mg).

Reaction of 1a with chloride 2e: The title compound was prepared according to the General Procedure D. The reaction of $1 \mathrm{a}(32.8 \mathrm{mg}, 0.20 \mathrm{mmol})$ and (E)-(2chlorovinyl)benzene $\mathbf{2 e}(49.7 \mathrm{mg}, 0.36 \mathrm{mmol})$ afforded 3a in $80 \%$ yield (40.0 $\mathrm{mg})$.

Reaction of 1a with iodide 2g: The title compound was prepared according to the General Procedure D. The reaction of $1 \mathrm{a}(32.8 \mathrm{mg}, 0.20 \mathrm{mmol})$ and (2iodovinyl)benzene $\mathbf{2 g}(82.8 \mathrm{mg}, 0.36 \mathrm{mmol}, \mathrm{E}: \mathrm{Z}=17: 1)$ afforded $3 \mathbf{a}$ in $52 \%$ yield $(26.0$ $\mathrm{mg})$.

${ }^{1}$ H NMR (400 MHz, CDCl 3$) \delta 7.37(\mathrm{~d}, J=7.2 \mathrm{~Hz}, 2 \mathrm{H})$, 7.32-7.24 (m, $\left.4 \mathrm{H}\right)$, 7.21-7.13 (m, $4 \mathrm{H}), 6.34(\mathrm{~d}, J=16.4 \mathrm{~Hz}, 1 \mathrm{H}), 6.23(\mathrm{~d}, J=16.4 \mathrm{~Hz}, 1 \mathrm{H}), 2.59-2.55(\mathrm{~m}, 2 \mathrm{H})$, 1.72-1.68 (m, $2 \mathrm{H}), 1.17$ (s, $6 \mathrm{H})$,.

${ }^{13}$ C NMR (100 MHz, CDCl 3$) \delta 143.2,140.2,138.0,128.6,128.4,128.3,126.9,126.3$, 126.1, 125.6, 45.3, 36.5, 31.4, 27.3.

IR (neat, cm-1): 3083, 3061, 3026, 3000, 2929, 2963, 1647, 1601, 1494, 1453, 1030, $746,695$.

(E)-(3,3-dimethylbut-1-en-1-yl)benzene (3b, known compound)

$\underbrace{\mathrm{Me}}_{\mathrm{Me}}$ The title compound was prepared according to the General $\mathrm{mg}, 0.40 \mathrm{mmol}$ ) with (E)-(2-bromovinyl)benzene 2a (36.4 mg, $0.20 \mathrm{mmol}) .{ }^{1} \mathrm{H}$ NMR and ${ }^{13} \mathrm{C}$ NMR data are consistent with those reported in reference 19.

$26.9 \mathrm{mg}, 84 \%$ yield, colorless oil.

${ }^{1}$ H NMR (400 MHz, CDCl3) $\delta 7.36(\mathrm{~d}, J=7.2 \mathrm{~Hz}, 2 \mathrm{H}), 7.28(\mathrm{t}, J=7.6 \mathrm{~Hz}, 2 \mathrm{H}), 7.18$ (t, $J=7.2 \mathrm{~Hz}, 1 \mathrm{H}), 6.31(\mathrm{~d}, J=16.4 \mathrm{~Hz}, 1 \mathrm{H}), 6.25(\mathrm{~d}, J=16.4 \mathrm{~Hz}, 1 \mathrm{H}), 1.12(\mathrm{~s}, 9 \mathrm{H})$.

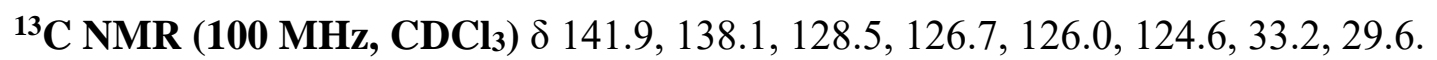
IR (neat, $\mathbf{c m}^{-1}$ ): 3082, 2960, 2095, 2866, 2737, 2702, 1475, 1362, 1161, 746, 670. 


\section{(E)-(3,3-dimethyldodec-1-en-1-yl)benzene (3c)}

$\mathrm{Me}$ The title compound was prepared according to the General
Procedure $\mathrm{D}$ from the reaction of 2-methylundecan-2-ol 1c (37.2 $\mathrm{mg}, 0.20 \mathrm{mmol})$ with (E)-(2-bromovinyl)benzene $2 \mathbf{a}(65.5 \mathrm{mg}, 0.36 \mathrm{mmol})$.

$38.1 \mathrm{mg}, 70 \%$ yield, colorless oil.

${ }^{1}$ H NMR (400 MHz, CDCl $) \delta 7.36(\mathrm{~d}, J=7.2 \mathrm{~Hz}, 2 \mathrm{H}), 7.29(\mathrm{t}, J=7.6 \mathrm{~Hz}, 2 \mathrm{H}), 7.18$ $(\mathrm{t}, J=7.6 \mathrm{~Hz}, 1 \mathrm{H}), 6.26(\mathrm{~d}, J=16.4 \mathrm{~Hz}, 1 \mathrm{H}), 6.18(\mathrm{~d}, J=16.4 \mathrm{~Hz}, 1 \mathrm{H}), 1.54-1.25(\mathrm{~m}$, $16 \mathrm{H}), 1.08(\mathrm{~s}, 6 \mathrm{H}), 0.87(\mathrm{t}, J=6.4 \mathrm{~Hz}, 3 \mathrm{H})$.

${ }^{13}$ C NMR (100 MHz, CDCl $) \delta 141.0,138.1,128.4,126.7,126.0,125.5,43.3,36.2$, $31.9,30.5,29.7,29.6,29.3,27.2,24.7,22.7,14.1$.

IR (neat, $\mathbf{c m}^{-1}$ ): 3082, 3060, 2927, 2855, 1494, 1030, 723, 692.

HRMS (EI): [M] ${ }^{+}$calcd for $\mathrm{C}_{20} \mathrm{H}_{32} 272.2504$, found 272.2498 .

(E)-(3-ethyl-3-methyldodec-1-en-1-yl)benzene (3d)

${ }^{\mathrm{Ph}}$ The title compound was prepared according to the General Procedure D from the reaction of 3-methyldodecan-3-ol 1d (40.0 mg, $0.20 \mathrm{mmol})$ with (E)-(2-bromovinyl)benzene $\mathbf{2 a}(65.5 \mathrm{mg}, 0.36 \mathrm{mmol})$.

$37.2 \mathrm{mg}, 65 \%$ yield, colorless oil.

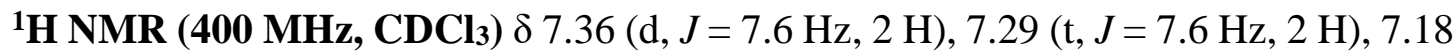
$(\mathrm{t}, J=7.6 \mathrm{~Hz}, 1 \mathrm{H}), 6.24(\mathrm{~d}, J=16.4 \mathrm{~Hz}, 1 \mathrm{H}), 6.09(\mathrm{~d}, J=16.4 \mathrm{~Hz}, 1 \mathrm{H}), 1.43-1.25(\mathrm{~m}$, $18 \mathrm{H}), 1.03(\mathrm{~s}, 3 \mathrm{H}), 0.87(\mathrm{t}, J=6.8 \mathrm{~Hz}, 3 \mathrm{H}), 0.82(\mathrm{t}, J=7.2 \mathrm{~Hz}, 3 \mathrm{H})$.

${ }^{13}$ C NMR (100 MHz, CDCl $) \delta 140.0,138.3,128.4,126.8,126.6,126.0,41.1,39.3$, $33.6,31.9,30.6,29.7,29.3,24.2,22.7,14.1,8.6,6.8,6.4$.

IR (neat, cm$^{-1}$ ): 3083, 3027, 2960, 2854, 1646, 1495, 1378, 1004, 745, 692.

HRMS (EI): $[\mathrm{M}]^{+}$calcd for $\mathrm{C}_{21} \mathrm{H}_{34} 286.2661$, found 286.2660 .

\section{(E)-(3-hexyl-3-methylnon-1-en-1-yl)benzene (3e)}

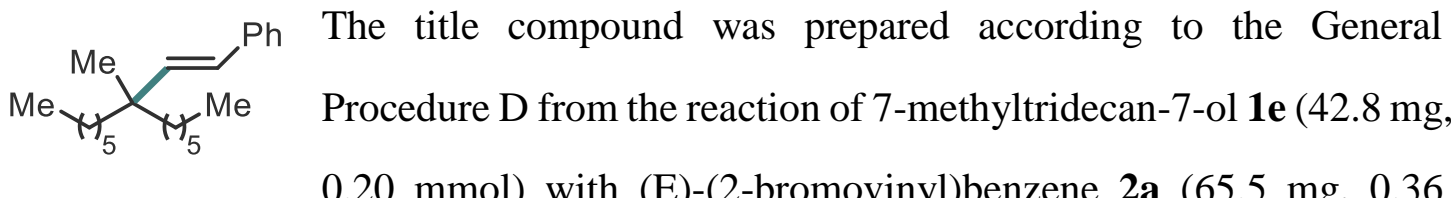
mmol). 
$36.0 \mathrm{mg}, 60 \%$ yield, colorless oil.

${ }^{1} \mathbf{H}$ NMR (400 MHz, CDCl 3$) \delta 7.36(\mathrm{~d}, J=7.2 \mathrm{~Hz}, 2 \mathrm{H}), 7.30(\mathrm{t}, J=7.6 \mathrm{~Hz}, 2 \mathrm{H}), 7.18$ $(\mathrm{t}, J=7.2 \mathrm{~Hz}, 1 \mathrm{H}), 6.23(\mathrm{~d}, J=16.4 \mathrm{~Hz}, 1 \mathrm{H}), 6.11(\mathrm{~d}, J=16.4 \mathrm{~Hz}, 1 \mathrm{H}), 1.37-1.20(\mathrm{~m}$, $20 \mathrm{H}), 1.04(\mathrm{~s}, 3 \mathrm{H}), 0.89-0.85$ (m, $6 \mathrm{H})$.

${ }^{13}$ C NMR (100 MHz, CDCl $) \delta 140.3,138.2,128.4,126.6,126.4,125.9,41.5,39.1$, $31.9,30.2,24.1,23.2,22.7,14.1$.

IR (neat, cm-1): 3082, 3027, 2957, 2929, 2857, 1464, 1377, 969, 746, 692.

HRMS (EI): [M] ${ }^{+}$calcd for $\mathrm{C}_{22} \mathrm{H}_{36} 300.2817$, found 300.2815.

((1E,11Z,14Z)-3,3-dimethylicosa-1,11,14-trien-1-yl)benzene (3f)

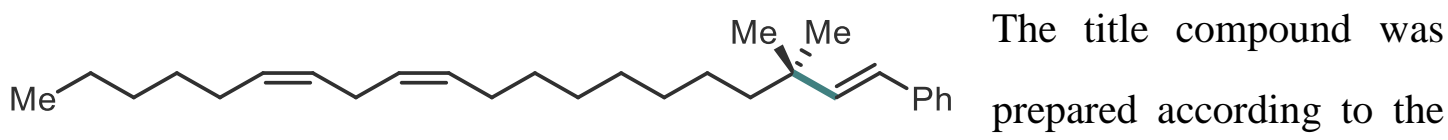

General Procedure D from the reaction of (10Z,13Z)-2-methylnonadeca-10,13-dien-2ol 1 f (58.9 mg, $0.20 \mathrm{mmol})$ with (E)-(2-bromovinyl)benzene 2a (65.5 mg, $0.36 \mathrm{mmol})$. $38.8 \mathrm{mg}, 51 \%$ yield, colorless oil.

${ }^{1}$ H NMR (600 MHz, CDCl3) $\delta 7.36(\mathrm{~d}, J=7.2 \mathrm{~Hz}, 2 \mathrm{H}), 7.29(\mathrm{t}, J=7.2 \mathrm{~Hz}, 2 \mathrm{H}), 7.18$ $(\mathrm{t}, J=7.2 \mathrm{~Hz}, 1 \mathrm{H}), 6.26(\mathrm{~d}, J=16.2 \mathrm{~Hz}, 1 \mathrm{H}), 6.18(\mathrm{~d}, J=16.2 \mathrm{~Hz}, 1 \mathrm{H}), 5.42-5.30(\mathrm{~m}$, 4 H), 2.78-2.70 (m, 2 H), 2.06-1.95 (m, 4 H), 1.36-1.24 (m, 18 H), 1.08 (s, 6 H), 0.90$0.87(\mathrm{~m}, 3 \mathrm{H})$.

${ }^{13}$ C NMR (400 MHz, CDCl3) $\delta$ 141.0, 138.1, 130.8, 130.2, 128.5, 127.9, 127.7, 126.7, $126.0,125.6,43.3,36.2,32.5,31.5,30.4,29.7,29.5,29.3,29.2,28.5,27.2$, 25.6, 24.7, 22.6, 14.1 .

IR (neat, cm-1): 2960, 2928, 2857, 1644, 1463, 987, 746, 693.

HRMS (ESI): [M+H] ${ }^{+}$calcd for $\mathrm{C}_{28} \mathrm{H}_{45} 381.3516$, found 381.3514 .

Ethyl (E)-6,6-dimethyl-8-phenyloct-7-enoate (3g)

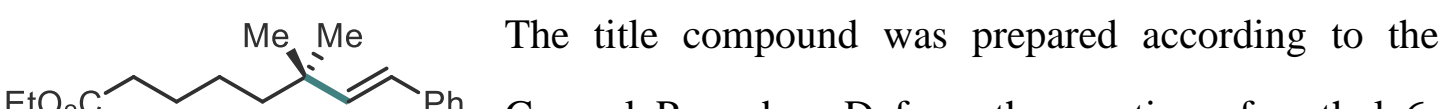

General Procedure D from the reaction of ethyl 6-

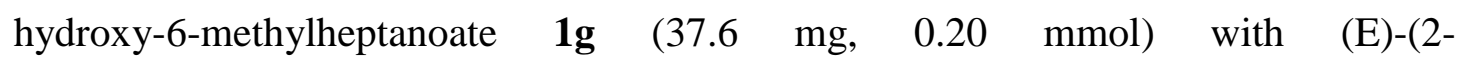
bromovinyl)benzene $2 \mathbf{a}(65.5 \mathrm{mg}, 0.36 \mathrm{mmol})$.

$33.5 \mathrm{mg}, 61 \%$ yield, colorless oil. 
${ }^{1} \mathbf{H}$ NMR (400 MHz, CDCl $) \delta 7.35(\mathrm{~d}, J=7.2 \mathrm{~Hz}, 2 \mathrm{H}), 7.29(\mathrm{t}, J=7.6 \mathrm{~Hz}, 2 \mathrm{H}), 7.18$ $(\mathrm{t}, J=7.2 \mathrm{~Hz}, 1 \mathrm{H}), 6.27(\mathrm{~d}, J=16.0 \mathrm{~Hz}, 1 \mathrm{H}), 6.16(\mathrm{~d}, J=16.0 \mathrm{~Hz}, 1 \mathrm{H}), 4.10(\mathrm{q}, J=$ $7.2 \mathrm{~Hz}, 2 \mathrm{H}), 2.28$ (t, $J=7.6 \mathrm{~Hz}, 2 \mathrm{H}), 1.64-1.56$ (m, $2 \mathrm{H}), 1.40-1.35$ (m, $2 \mathrm{H}), 1.33-$ $1.25(\mathrm{~m}, 2 \mathrm{H}), 1.22(\mathrm{t}, J=7.2 \mathrm{~Hz}, 3 \mathrm{H}), 1.08(\mathrm{~s}, 6 \mathrm{H})$.

${ }^{13}$ C NMR (100 MHz, CDCl 3$) \delta 173.8,140.5,138.0,128.4,126.7,126.0,125.8,60.1$, $42.8,36.2,34.3,27.2,25.7,24.3,14.2$.

IR (neat, cm-1): 2036, 2959, 2867, 1736, 1644, 1181, 1031, 970, 748, 694.

HRMS (ESI): $[\mathrm{M}+\mathrm{H}]^{+}$calcd for $\mathrm{C}_{18} \mathrm{H}_{27} \mathrm{O}_{2} 275.2006$, found 275.1998.

(E)-1-(3,3-dimethyl-5-phenylpent-4-en-1-yl)-4-fluorobenzene (3h)

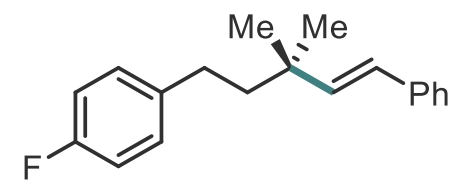

The title compound was prepared according to the General

Procedure D from the reaction of 4-(4-fluorophenyl)-2methylbutan-2-ol $\mathbf{1 h}(36.4 \mathrm{mg}, 0.20 \mathrm{mmol})$ with (E)-(2bromovinyl)benzene $\mathbf{2 a}(65.5 \mathrm{mg}, 0.36 \mathrm{mmol})$.

$40.8 \mathrm{mg}, 76 \%$ yield, colorless oil.

${ }^{1}$ H NMR (400 MHz, CDCl3) $\delta 7.37$ (d, $\left.J=7.6 \mathrm{~Hz}, 2 \mathrm{H}\right), 7.30(\mathrm{t}, J=7.6 \mathrm{~Hz}, 2 \mathrm{H}), 7.20$ (t, $J=7.2 \mathrm{~Hz}, 1 \mathrm{H}), 7.11-7.08(\mathrm{~m}, 2 \mathrm{H}), 6.93(\mathrm{t}, J=8.8 \mathrm{~Hz}, 2 \mathrm{H}), 6.34(\mathrm{~d}, J=16.0 \mathrm{~Hz}$, $1 \mathrm{H}), 6.21(\mathrm{~d}, J=16.0 \mathrm{~Hz}, 1 \mathrm{H}), 2.55-2.51(\mathrm{~m}, 2 \mathrm{H}), 1.68-1.64(\mathrm{~m}, 2 \mathrm{H}), 1.16(\mathrm{~s}, 6 \mathrm{H})$.

${ }^{13}$ C NMR (100 MHz, CDCl $) \delta 161.1(\mathrm{~d}, J=241 \mathrm{~Hz}), 140.0,138.7(\mathrm{~d}, J=3.0 \mathrm{~Hz})$, $137.8,129.5(\mathrm{~d}, J=8.0 \mathrm{~Hz}), 128.5,126.9,126.3,126.0,115.0(\mathrm{~d}, J=21 \mathrm{~Hz}), 45.4,36.4$, $30.5,27.2$.

${ }^{19}$ F NMR (376 MHz, CDCl3): $\delta$-118.1.

IR (neat, $\mathbf{c m}^{-1}$ ): 3082, 3026, 2960, 2866, 1648, 1509, 1471, 1379, 1220, 1157, 1029, 970, 854, 748, 694 .

HRMS (EI): [M] ${ }^{+}$calcd for $\mathrm{C}_{19} \mathrm{H}_{21} \mathrm{~F} 268.1627$, found 268.1621 .

(E)-5-(3,3-dimethyl-5-phenylpent-4-en-1-yl)benzo[d][1,3]dioxole (3i)

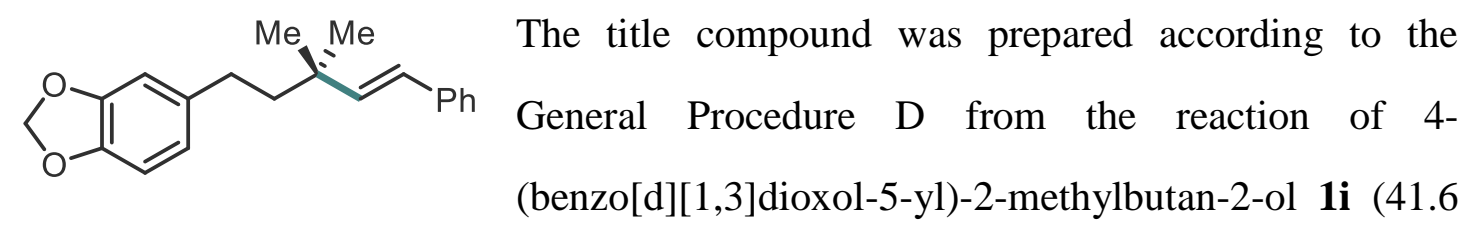
$\mathrm{mg}, 0.20 \mathrm{mmol})$ with (E)-(2-bromovinyl)benzene $\mathbf{2 a}(65.5 \mathrm{mg}, 0.36 \mathrm{mmol})$. 
$45.9 \mathrm{mg}, 78 \%$ yield, colorless oil.

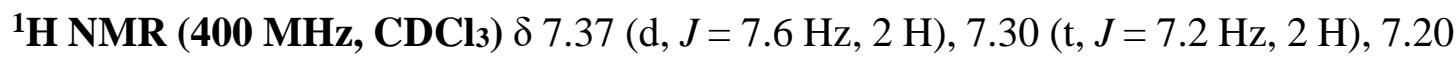
$(\mathrm{t}, J=7.2 \mathrm{~Hz}, 1 \mathrm{H}), 6.71(\mathrm{~d}, J=8.0 \mathrm{~Hz}, 1 \mathrm{H}), 6.66(\mathrm{~s}, 1 \mathrm{H}), 6.61(\mathrm{~d}, J=8.0 \mathrm{~Hz}, 1 \mathrm{H})$, $6.33(\mathrm{~d}, J=16.4 \mathrm{~Hz}, 1 \mathrm{H}), 6.21(\mathrm{~d}, J=16.0 \mathrm{~Hz}, 1 \mathrm{H}), 5.89$ (s, $2 \mathrm{H}), 2.51-2.47$ (m, $2 \mathrm{H}$ ), 1.67-1.63 (m, $2 \mathrm{H}), 1.16$ (s, $6 \mathrm{H})$,.

${ }^{13}$ C NMR (100 MHz, CDCl3) $\delta$ 147.5, 145.4, 140.1, 137.9, 137.0, 128.5, 126.9, 126.2, $126.1,120.9,108.8,108.1,100.7,45.6,36.4,31.1,27.2$.

IR (neat, cm$^{-1}$ ): 3026, 2959, 2776, 1642, 1606, 1442, 1246, 1188, 1072, 970, 938, 859, 748,694

HRMS (ESI): $[\mathrm{M}+\mathrm{H}]^{+}$calcd for $\mathrm{C}_{20} \mathrm{H}_{23} \mathrm{O}_{2}$ 295.1693, found 295.1692.

(E)-4-(3,3-dimethyl-5-phenylpent-4-en-1-yl)phenyl pivalate (3j)<smiles>CC(C)(C)CCc1ccc(Oc2ccccc2)cc1</smiles>
The title compound was prepared according to the General Procedure D from the reaction of 4-(3hydroxy-3-methylbutyl)phenyl pivalate $\mathbf{1 j}$ (52.8 $\mathrm{mg}$, $0.20 \mathrm{mmol})$ with (E)-(2-bromovinyl)benzene $2 \mathbf{a}(65.5 \mathrm{mg}, 0.36 \mathrm{mmol})$.

$53.9 \mathrm{mg}, 77 \%$ yield, colorless oil.

${ }^{1} \mathbf{H}$ NMR $\left(600 \mathrm{MHz}, \mathbf{C D C l}_{3}\right) \delta 7.37(\mathrm{~d}, J=7.2 \mathrm{~Hz}, 2 \mathrm{H}), 7.30(\mathrm{t}, J=7.8 \mathrm{~Hz}, 2 \mathrm{H}), 7.24-$ $7.19(\mathrm{~m}, 1 \mathrm{H}), 7.15(\mathrm{~d}, J=8.4 \mathrm{~Hz}, 2 \mathrm{H}), 6.94(\mathrm{~d}, J=8.4 \mathrm{~Hz}, 2 \mathrm{H}), 6.34$ (d, $J=16.2 \mathrm{~Hz}$, $1 \mathrm{H}), 6.22(\mathrm{~d}, J=16.2 \mathrm{~Hz}, 1 \mathrm{H}), 2.57-2.54(\mathrm{~m}, 2 \mathrm{H}), 1.70-1.67(\mathrm{~m}, 2 \mathrm{H}), 1.34(\mathrm{~s}, 9 \mathrm{H})$, $1.17(\mathrm{~s}, 6 \mathrm{H})$.

${ }^{13}$ C NMR (150 MHz, CDCl 3$) \delta 177.2,149.0,140.4,140.0,137.9,129.1,128.5,126.9$, 126.3, 126.0, 121.2, 45.2, 39.0, 36.4, 30.7, 27.2, 27.1.

IR (neat, $\mathbf{c m}^{-1}$ ): 3062, 3029, 2965, 1751, 1646, 1508, 1366, 1279, 1230, 1167, 1130, $971,747$.

HRMS (ESI): [M+Na] ${ }^{+}$calcd for $\mathrm{C}_{24} \mathrm{H}_{30} \mathrm{NaO}_{2} 373.2138$, found 373.2138.

(E)-(4-(3,3-dimethyl-5-phenylpent-4-en-1-yl)phenyl)trimethylsilane (3k)<smiles>CC(C)(C=Cc1ccccc1)CCc1ccc(S(C)(=O)=O)cc1</smiles>
The title compound was prepared according to the General Procedure D from the reaction of 2-methyl-4(4-(trimethylsilyl)phenyl)butan-2-ol 1k (47.2 mg, 0.20 
mmol) with (E)-(2-bromovinyl)benzene 2a $(65.5 \mathrm{mg}, 0.36 \mathrm{mmol})$.

$45.1 \mathrm{mg}, 70 \%$ yield, colorless oil.

${ }^{1} \mathbf{H}$ NMR (400 MHz, CDCl $) \delta 7.43(\mathrm{~d}, J=8.0 \mathrm{~Hz}, 2 \mathrm{H}), 7.38-7.36(\mathrm{~m}, 2 \mathrm{H}), 7.30(\mathrm{t}, J$ $=7.6 \mathrm{~Hz}, 2 \mathrm{H}), 7.21-7.16(\mathrm{~m}, 3 \mathrm{H}), 6.34(\mathrm{~d}, J=16.0 \mathrm{~Hz}, 1 \mathrm{H}), 6.22(\mathrm{~d}, J=16.0 \mathrm{~Hz}, 1$ H), 2.58-2.54 (m, 2 H), 1.72-1.68 (m, 2 H), 1.17 (s, $6 \mathrm{H}), 0.24$ (s, 9 H),.

${ }^{13}$ C NMR (100 MHz, CDCl $) \delta$ 143.8, 140.1, 137.9, 137.1, 133.4, 128.5, 127.8, 126.9, $126.2,126.1,45.2,36.5,31.3,27.2,-1.1$.

IR (neat, $\mathbf{c m}^{-1}$ ): 3063, 3026, 2957, 2863, 1648, 1553, 1450, 1398, 1308, 1108, 969, $852,838,747,693$.

HRMS (ESI): $[\mathrm{M}+\mathrm{H}]^{+}$calcd for $\mathrm{C}_{22} \mathrm{H}_{31} \mathrm{Si} 323.2190$, found 323.2197.

(E)-4-(3,3-dimethyl-5-phenylpent-4-en-1-yl)phenol (3I)<smiles>CC(C)(/C=C/c1ccccc1)CCc1ccc(O)cc1</smiles>

The title compound was prepared according to the General Procedure D from the reaction of 4-(3-hydroxy3-methylbutyl)phenol 11 (36.0 mg, $0.20 \mathrm{mmol})$ with (E)(2-bromovinyl)benzene $\mathbf{2 a}(65.5 \mathrm{mg}, 0.36 \mathrm{mmol})$.

$35.1 \mathrm{mg}, 66 \%$ yield, colorless oil.

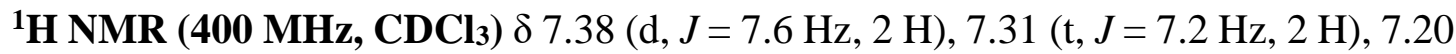
(t, $J=7.2 \mathrm{~Hz}, 1 \mathrm{H}), 7.03(\mathrm{~d}, J=8.4 \mathrm{~Hz}, 2 \mathrm{H}), 6.73(\mathrm{~d}, J=8.4 \mathrm{~Hz}, 2 \mathrm{H}), 6.33$ (d, $J=$ $16.2 \mathrm{~Hz}, 1 \mathrm{H}), 6.22(\mathrm{~d}, J=16.2 \mathrm{~Hz}, 1 \mathrm{H}), 4.68(\mathrm{~s}, 1 \mathrm{H}), 2.52-2.47(\mathrm{~m}, 2 \mathrm{H}), 1.68-1.63(\mathrm{~m}$, $2 \mathrm{H}), 1.16(\mathrm{~s}, 6 \mathrm{H})$.

${ }^{13}$ C NMR (100 MHz, CDCl $)$ ) $\delta 153.4,140.2,137.9,135.4,129.3,128.5,126.8,126.2$, 126.0, 115.1, 45.5, 36.4, 30.4, 27.2.

IR (neat, cm$^{-1}$ ): 3366, 2960, 2932, 1614, 1597, 1448, 1226, 1172, 1094, 969, 828, 794, 744, 714, 693.

HRMS (ESI): $[\mathrm{M}+\mathrm{Na}]^{+}$calcd for $\mathrm{C}_{19} \mathrm{H}_{22} \mathrm{NaO} 289.1563$, found 289.1566. 
(3m)<smiles>CC(C)(/C=C/c1ccccc1)CCc1ccc(O)cc1</smiles>

The title compound was prepared according to the

General Procedure D from the reaction of 4-(3-hydroxy3-methylbutyl)phenyl trifluoromethanesulfonate $\mathbf{1 m}$ (62.4 mg, $0.20 \mathrm{mmol}$ ) with (E)-(2-bromovinyl)benzene $\mathbf{2 a}$ (65.5 mg, $0.36 \mathrm{mmol})$.

$47.8 \mathrm{mg}, 60 \%$ yield, colorless oil.

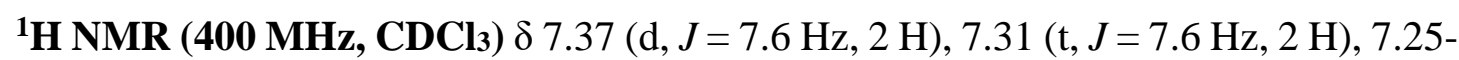
$7.19(\mathrm{~m}, 3 \mathrm{H}), 7.15(\mathrm{~d}, J=8.4 \mathrm{~Hz}, 2 \mathrm{H}), 6.35(\mathrm{~d}, J=16.4 \mathrm{~Hz}, 1 \mathrm{H}), 6.20(\mathrm{~d}, J=16.4$ Hz, 1 H), 2.61-2.57 (m, 2 H), 1.71-1.66 (m, 2 H), 1.18 (s, 6 H).

${ }^{13}$ C NMR (100 MHz, CDCl3) $\delta$ 147.6, 143.7, 139.7, 137.7, 130.0, 128.5, 127.0, 126.6, 126.1, 121.1, 118.7 (q, $J=315 \mathrm{~Hz}), 45.0,36.4,30.8,27.2$.

${ }^{19}$ F NMR (376 MHz, CDCl 3$): ~ \delta-72.9$

IR (neat, $\mathbf{c m}^{-1}$ ): 3028, 2963, 2868, 1646, 1448, 1366, 1265, 1215, 1142, 1180, 1097 , 971, 891, 695.

HRMS (ESI): $[\mathrm{M}+\mathrm{H}]^{+}$calcd for $\mathrm{C}_{20} \mathrm{H}_{22} \mathrm{~F}_{3} \mathrm{O}_{3} \mathrm{~S} 399.1236$, found 399.1239.

\section{(E)-1-bromo-4-(3,3-dimethyl-5-phenylpent-4-en-1-yl)benzene (3n)}

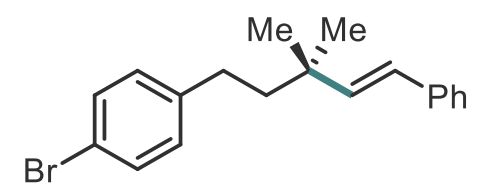

The title compound was prepared according to the

General Procedure D from the reaction of 4-(4bromophenyl)-2-methylbutan-2-ol $\mathbf{1 n}$ (48.4 $\mathrm{mg}, 0.20$ mmol) with (E)-(2-bromovinyl)benzene $2 \mathbf{a}(65.5 \mathrm{mg}, 0.36 \mathrm{mmol})$.

$51.8 \mathrm{mg}, 79 \%$ yield, colorless oil.

${ }^{1} \mathbf{H}$ NMR (400 MHz, CDCl$)$ ) $\delta$ 7.37-7.32 (m, 4 H), 7.31 (t, $J=7.6$ Hz, 2 H), 7.24-7.18 $(\mathrm{m}, 1 \mathrm{H}), 7.03(\mathrm{~d}, J=8.4 \mathrm{~Hz}, 2 \mathrm{H}), 6.33(\mathrm{~d}, J=16.4 \mathrm{~Hz}, 1 \mathrm{H}), 6.20(\mathrm{~d}, J=16.4 \mathrm{~Hz}, 1$ H), 2.54-2.49 (m, 2 H), 1.68-1.63 (m, $2 \mathrm{H}), 1.16$ (s, $6 \mathrm{H})$.

${ }^{13}$ C NMR (100 MHz, CDCl 3$) \delta 142.1,139.9,137.8,131.3,130.1,128.5,126.9,126.4$, 126.0, 119.3, 45.1, 36.4, 30.8, 27.2.

IR (neat, $\mathbf{c m}^{-1}$ ): 3025, 2960, 2864, 1488, 1450, 1095, 1028, 1011, 833, 747, 693.

HRMS (EI): [M] ${ }^{+}$calcd for $\mathrm{C}_{19} \mathrm{H}_{21} \mathrm{Br} 328.0827$, found 328.0827 . 
(E)-(6-chloro-3,3-dimethylhex-1-en-1-yl)benzene (3o)

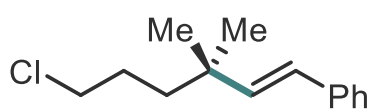

The title compound was prepared according to the General

Procedure D from the reaction of 5-chloro-2-methylpentan-2-

ol 10 (27.2 mg, $0.20 \mathrm{mmol})$ with (E)-(2-bromovinyl)benzene $2 \mathbf{a}(65.5 \mathrm{mg}, 0.36 \mathrm{mmol})$. $29.8 \mathrm{mg}, 67 \%$ yield, colorless oil.

${ }^{1}$ H NMR (400 MHz, CDCl $) \delta 7.35(\mathrm{~d}, J=7.6 \mathrm{~Hz}, 2 \mathrm{H}), 7.29(\mathrm{t}, J=7.2 \mathrm{~Hz}, 2 \mathrm{H}), 7.19$ $(\mathrm{t}, J=7.2 \mathrm{~Hz}, 1 \mathrm{H}), 6.29(\mathrm{~d}, J=16.0 \mathrm{~Hz}, 1 \mathrm{H}), 6.15(\mathrm{~d}, J=16.4 \mathrm{~Hz}, 1 \mathrm{H}), 3.51(\mathrm{t}, J=$ $6.8 \mathrm{~Hz}, 2 \mathrm{H}), 1.78-1.71(\mathrm{~m}, 2 \mathrm{H}), 1.52-1.48(\mathrm{~m}, 2 \mathrm{H}), 1.11$ (s, $6 \mathrm{H})$.

${ }^{13}$ C NMR (100 MHz, CDCl $) \delta 139.8,137.8,128.5,126.9,126.3,126.0,45.8,40.3$, $36.0,28.3,27.2$.

IR (neat, $\mathbf{c m}^{-1}$ ): 3082, 3059, 2959, 2869, 1578, 1546, 1385, 1310, 1073, 1029, 785, $717,694$.

HRMS (EI): [M] ${ }^{+}$calcd for $\mathrm{C}_{14} \mathrm{H}_{19} \mathrm{Cl} 222.1175$, found 222.1188 .

\section{(E)-but-1-ene-1,3-diyldibenzene (3p, known compound)}<smiles>CC(/C=C/c1ccccc1)c1ccccc1</smiles>

The title compound was prepared according to the General Procedure D from the reaction of 1-phenylethan-1-ol 1p (24.4 mg, $0.20 \mathrm{mmol})$ with (E)-(2-bromovinyl)benzene $2 \mathbf{a}(65.5 \mathrm{mg}, 0.36$ mmol). ${ }^{1} \mathrm{H}$ NMR and ${ }^{13} \mathrm{C}$ NMR data are consistent with those reported in reference 20. $14.2 \mathrm{mg}, 34 \%$ yield, colorless oil.

$1 H$ NMR (400 MHz, CDCl3) :7.52-7.25 (m, 10H), 6.52 (d, $J=15.3,1 \mathrm{H}), 6.46(\mathrm{~d}, J=$ $15.3,1 \mathrm{H}), 3.74(\mathrm{~m}, 1 \mathrm{H}), 1.57(\mathrm{~d}, \mathrm{~J}=7.0,3 \mathrm{H})$.

13C NMR (100 MHz, CDCl3) $\delta$ 145.6, 137.5, 135.2, 128.5, 128.4, 127.3, 127.0, 126.2, $126.1,126.0,42.5,21.2$.

(E)-(2-(1-methylcyclopentyl)vinyl)benzene (3q)

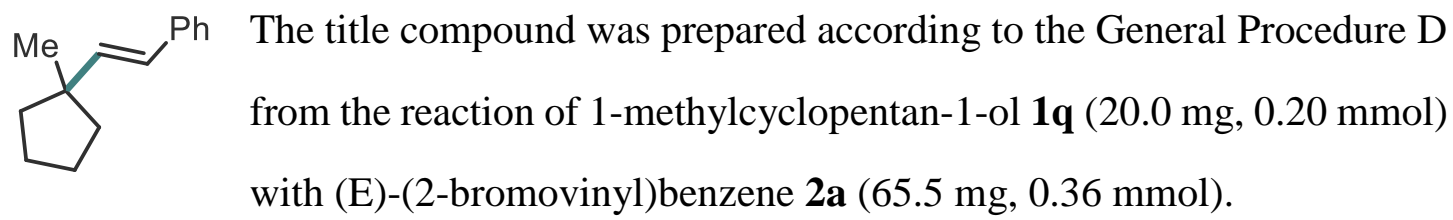

$22.7 \mathrm{mg}, 61 \%$ yield, colorless oil.

${ }^{1}$ H NMR (400 MHz, CDCl 3$) \delta 7.36(\mathrm{~d}, J=7.6 \mathrm{~Hz}, 2 \mathrm{H}), 7.29(\mathrm{t}, J=7.6 \mathrm{~Hz}, 2 \mathrm{H}), 7.18$ 
(t, J= 7.2 Hz, $1 \mathrm{H}), 6.36-6.27$ (m, $2 \mathrm{H}), 1.74-1.65$ (m, $6 \mathrm{H}), 1.49-1.45$ (m, $2 \mathrm{H}), 1.16$ (s, $3 \mathrm{H})$.

${ }^{13}$ C NMR (100 MHz, CDCl3) $\delta$ 140.7, 138.1, 128.5, 126.7, 126.0, 125.0, 44.8, 39.6, 25.8, 24.2 .

IR (neat, $\mathbf{c m}^{-1}$ ): 2952, 2913, 2369, 2343, 1654, 1638, 1250, 1176, 1092, 1034, 731.

HRMS (ESI): $[\mathrm{M}+\mathrm{H}]^{+}$calcd for $\mathrm{C}_{14} \mathrm{H}_{19}$ 187.1481, found 187.1472.

\section{(E)-4-methyl-4-styryltetrahydro-2H-pyran (3r)}

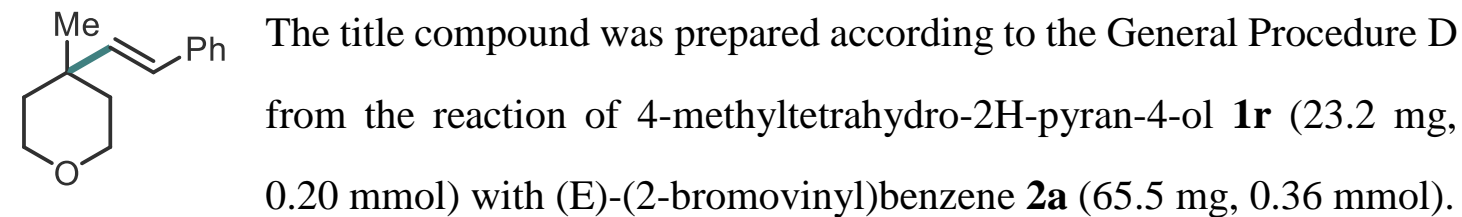
$25.5 \mathrm{mg}, 63 \%$ yield, colorless oil.

${ }^{1} \mathbf{H}$ NMR (600 MHz, CDCl 3$) \delta 7.37(\mathrm{~d}, J=7.8 \mathrm{~Hz}, 2 \mathrm{H}), 7.31(\mathrm{t}, J=7.2 \mathrm{~Hz}, 2 \mathrm{H}), 7.22$ (t, $J=7.2 \mathrm{~Hz}, 1 \mathrm{H}), 6.35(\mathrm{~d}, J=16.8 \mathrm{~Hz}, 1 \mathrm{H}), 6.20(\mathrm{~d}, J=16.2 \mathrm{~Hz}, 1 \mathrm{H}), 3.76-3.68(\mathrm{~m}$, $4 \mathrm{H}), 1.76-1.73$ (m, $2 \mathrm{H}), 1.59-1.55$ (m, $2 \mathrm{H}) 1.17$ (s, $3 \mathrm{H})$.

${ }^{13}$ C NMR (150 MHz, CDCl3) $\delta 139.1,137.7,128.5,127.2,127.1,126.0,64.3,37.8$, 34.0, 27.3.

IR (neat, $\mathbf{c m}^{-1}$ ): 3082, 3026, 2927, 2855, 1643, 1578, 1241, 1168, 1137, 1073, 1035, 1016, 885, 748, 694.

HRMS (ESI): $[\mathrm{M}+\mathrm{H}]^{+}$calcd for $\mathrm{C}_{14} \mathrm{H}_{19} \mathrm{O} 203.1430$, found 203.1433 .

\section{(E)-(2-(1-methylcyclohexyl)vinyl)benzene (3s, known compound)}

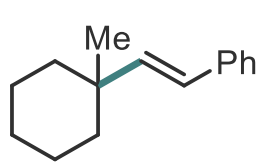

The title compound was prepared according to the General Procedure D from the reaction of 1-methylcyclohexan-1-ol 1s $(22.8 \mathrm{mg}, 0.20$ mmol) with (E)-(2-bromovinyl)benzene 2a (65.5 mg, $0.36 \mathrm{mmol})$.

$24.8 \mathrm{mg}$, 62\% yield, colorless oil. ${ }^{1} \mathrm{H}$ NMR and ${ }^{13} \mathrm{C}$ NMR data are consistent with those reported in reference 21 .

${ }^{1} \mathbf{H}$ NMR (400 MHz, CDCl3) $\delta 7.37(\mathrm{~d}, J=7.2 \mathrm{~Hz}, 2 \mathrm{H}), 7.29(\mathrm{t}, J=7.6 \mathrm{~Hz}, 2 \mathrm{H}), 7.18$ $(\mathrm{t}, J=7.2 \mathrm{~Hz}, 1 \mathrm{H}), 6.32(\mathrm{~d}, J=16.4 \mathrm{~Hz}, 1 \mathrm{H}), 6.21(\mathrm{~d}, J=16.4 \mathrm{~Hz}, 1 \mathrm{H}), 1.64-1.59(\mathrm{~m}$, $2 \mathrm{H}), 1.58-1.48$ (m, $4 \mathrm{H}), 1.43-1.34$ (m, $4 \mathrm{H}), 1.07$ (s, $3 \mathrm{H})$.

${ }^{13}$ C NMR (100 MHz, CDCl3) $\delta 141.0,138.2,128.5,126.7,125.9$ (2C), 38.0, 36.2, 27.6, 
$26.3,22.4$.

IR (neat, $\mathbf{c m}^{-1}$ ): 3082, 2926, 2851, 1492, 1029, 877, 850, 747, 692.

(E)-1-methyl-1-styrylcycloheptane (3t)

$\mathrm{Ph}$ The title compound was prepared according to the General
Procedure $\mathrm{D}$ from the reaction of 1-methylcycloheptan-1-ol 1t (25.6 $\mathrm{mg}, 0.20 \mathrm{mmol}$ ) with (E)-(2-bromovinyl)benzene $2 \mathbf{a}(65.5 \mathrm{mg}, 0.36 \mathrm{mmol})$.

$23.6 \mathrm{mg}$, 55\% yield, colorless oil.

${ }^{1}$ H NMR (400 MHz, CDCl 3$) \delta 7.36(\mathrm{~d}, J=7.2 \mathrm{~Hz}, 2 \mathrm{H}), 7.29(\mathrm{t}, J=7.2 \mathrm{~Hz}, 2 \mathrm{H}), 7.18$ $(\mathrm{t}, J=7.2 \mathrm{~Hz}, 1 \mathrm{H}), 6.31(\mathrm{~d}, J=16.4 \mathrm{~Hz}, 1 \mathrm{H}), 6.25(\mathrm{~d}, J=16.4 \mathrm{~Hz}, 1 \mathrm{H}), 1.79-1.69(\mathrm{~m}$, $2 \mathrm{H}), 1.55-1.46(\mathrm{~m}, 10 \mathrm{H}), 1.07$ (s, $3 \mathrm{H})$.

${ }^{13}$ C NMR (100 MHz, CDCl3) $\delta 142.0,138.3,128.5,126.6,126.0,124.7,40.5,39.3$, $30.4,29.0,23.1$.

IR (neat, cm$^{-1}$ ): 3082, 3060, 3026, 2921, 2853, 1802, 1748, 1643, 1029, 908, 807, 746, 692.

HRMS (ESI): $[\mathrm{M}+\mathrm{H}]^{+}$calcd for $\mathrm{C}_{16} \mathrm{H}_{23} 215.1794$, found 215.1781 .

(1R,3S,5r,7r)-2-methyl-2-((E)-styryl)adamantane (3u)

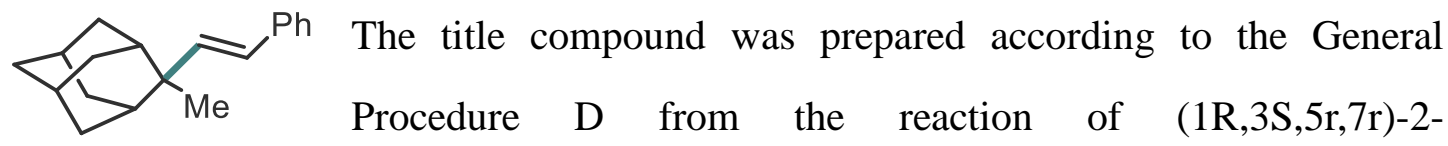
methyladamantan-2-ol 1u (33.2 mg, $0.20 \mathrm{mmol})$ with (E)-(2-bromovinyl)benzene 2a (65.5 mg, $0.36 \mathrm{mmol})$.

$25.7 \mathrm{mg}, 51 \%$ yield, colorless oil.

${ }^{1} \mathbf{H}$ NMR (600 MHz, CDCl$) \delta 7.37(\mathrm{~d}, J=7.2 \mathrm{~Hz}, 2 \mathrm{H}), 7.30(\mathrm{t}, J=7.8 \mathrm{~Hz}, 2 \mathrm{H}), 7.18$ $(\mathrm{t}, J=7.2 \mathrm{~Hz}, 1 \mathrm{H}), 6.32(\mathrm{~d}, J=16.8 \mathrm{~Hz}, 1 \mathrm{H}), 6.28(\mathrm{~d}, J=16.8 \mathrm{~Hz}, 1 \mathrm{H}), 2.15-2.09(\mathrm{~m}$, $4 \mathrm{H}), 1.87-1.54(\mathrm{~m}, 10 \mathrm{H}), 1.18(\mathrm{~s}, 3 \mathrm{H})$.

${ }^{13}$ C NMR (100 MHz, CDCl $) \delta 142.4,138.4,128.5,126.6,126.1,125.7,53.4,41.3$, $38.9,36.5,34.1,32.9,28.0,27.9,27.0$.

IR (neat, $\mathbf{c m}^{-1}$ ): 3083, 3060, 3027, 2986, 2909, 2863, 1601, 1493, 1448, 1377, 1108, 1071, 964, 930, 803, 747, 691.

HRMS (EI): [M] ${ }^{+}$calcd for $\mathrm{C}_{19} \mathrm{H}_{24} 252.1878$, found 252.1866 . 


\section{(3r,5r,7r)-1-((E)-styryl)adamantane (3v, known compound)}

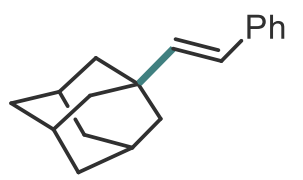

The title compound was prepared according to the General Procedure D from the reaction of $(3 \mathrm{~s}, 5 \mathrm{~s}, 7 \mathrm{~s})$-adamantan-1-ol $\mathbf{1 v}$ (30.4 mg, $0.20 \mathrm{mmol}$ ) with (E)-(2-bromovinyl)benzene 2a (65.5 mg, $0.36 \mathrm{mmol}) .{ }^{1} \mathrm{H} \mathrm{NMR}$ and ${ }^{13} \mathrm{C} \mathrm{NMR}$ data are consistent with those reported in reference 21.

$11.0 \mathrm{mg}, 23 \%$ yield, colorless oil.

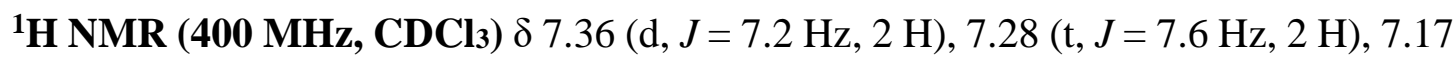
$(\mathrm{t}, J=7.2 \mathrm{~Hz}, 1 \mathrm{H}), 6.24(\mathrm{~d}, J=16.2 \mathrm{~Hz}, 1 \mathrm{H}), 6.10(\mathrm{~d}, J=16.2 \mathrm{~Hz}, 1 \mathrm{H}), 2.03-1.68(\mathrm{~m}$, $15 \mathrm{H})$.

${ }^{13}$ C NMR (100 MHz, CDCl $) \delta 142.1,138.2,128.4,126.7,126.0,124.5,42.2,36.9$, $35.2,28.5$.

(E)-1-(3,3-dimethyl-5-phenylpent-1-en-1-yl)-4-methylbenzene (3w)<smiles>Cc1ccc(/C=C/C(C)(C)CCc2ccccc2)cc1</smiles>
The title compound was prepared according to the General Procedure D from the reaction of 2-methyl4-phenylbutan-2-ol 1a (32.8 mg, $0.20 \mathrm{mmol})$ with 1(2-fluorovinyl)-4-methylbenzene $2 \mathbf{d}(E / Z=1: 4,49.0 \mathrm{mg}, 0.36 \mathrm{mmol})$.

$37.5 \mathrm{mg}, 71 \%$ yield, colorless oil.

${ }^{1}$ H NMR (400 MHz, CDCl3) $\delta$ 7.28-7.23 (m, 4 H), $7.16(\mathrm{~d}, J=7.2 \mathrm{~Hz}, 3 \mathrm{H}), 7.11(\mathrm{~d}$, $J=7.6 \mathrm{~Hz}, 2 \mathrm{H}), 6.31(\mathrm{~d}, J=16.4 \mathrm{~Hz}, 1 \mathrm{H}), 6.17(\mathrm{~d}, J=16.4 \mathrm{~Hz}, 1 \mathrm{H}), 2.58-2.54(\mathrm{~m}, 2$ H), $2.32(\mathrm{~s}, 3 \mathrm{H}), 1.71-1.66(\mathrm{~m}, 2 \mathrm{H}), 1.16(\mathrm{~s}, 6 \mathrm{H})$.

${ }^{13}$ C NMR (100 MHz, CDCl3) $\delta$ 143.2, 139.2, 136.5, 135.2, 129.2, 128.3 (2C), 126.1, 126.0, 125.5, 45.4, 36.4, 31.4, 27.3, 21.1.

IR (neat, $\mathbf{c m}^{-1}$ ): 3029, 2961, 2868, 1899, 1802, 1735, 1701, 1686, 1649, 1605, 1515, 1455, 1384, 1364, 1205, 1183, 1108, 1073, 1030, 971, 857, 800, 760, 740, 699.

HRMS (ESI): $[\mathrm{M}+\mathrm{H}]^{+}$calcd for $\mathrm{C}_{20} \mathrm{H}_{25}$ 265.1951, found 265.1953 . 

compound)<smiles>CC(C)(/C=C/c1ccc(F)cc1)CCc1ccccc1</smiles>

The title compound was prepared according to the General Procedure D from the reaction of 2-methyl-4phenylbutan-2-ol 1a (32.8 mg, $0.20 \mathrm{mmol})$ with (E)-1(2-chlorovinyl)-4-fluorobenzene $2 \mathbf{2 f}(56.2 \mathrm{mg}, 0.36 \mathrm{mmol}) .{ }^{1} \mathrm{H}$ NMR and ${ }^{13} \mathrm{C}$ NMR data are consistent with those reported in reference 7.

$45.1 \mathrm{mg}, 84 \%$ yield, colorless oil.

${ }^{1}$ H NMR (400 MHz, CDCl 3 ) $\delta$ 7.34-7.23 (m, 4 H), 7.18-7.16 (m, 3 H), 6.99 (t, $J=8.8$ $\mathrm{Hz}, 2 \mathrm{H}), 6.30(\mathrm{~d}, J=16.4 \mathrm{~Hz}, 1 \mathrm{H}), 6.14(\mathrm{~d}, J=16.0 \mathrm{~Hz}, 1 \mathrm{H}), 2.58-2.54(\mathrm{~m}, 2 \mathrm{H})$, 1.71-1.67 (m, $2 \mathrm{H}), 1.16$ (s, $6 \mathrm{H})$.

${ }^{13}$ C NMR (100 MHz, CDCl $) \delta 161.9(\mathrm{~d}, J=244 \mathrm{~Hz}), 143.1,139.9,134.0(\mathrm{~d}, J=3 \mathrm{~Hz})$, 128.3, 128.2, $127.4(\mathrm{~d}, J=8 \mathrm{~Hz}), 125.6,125.1,115.3(\mathrm{~d}, J=21 \mathrm{~Hz}), 45.2,36.4,31.3$, 27.2.

${ }^{19}$ F NMR (376 MHz, CDCl3): $\delta-115.8$

\section{(E)-2-(3,3-dimethyl-5-phenylpent-1-en-1-yl)-1,3-dimethylbenzene (3y)}

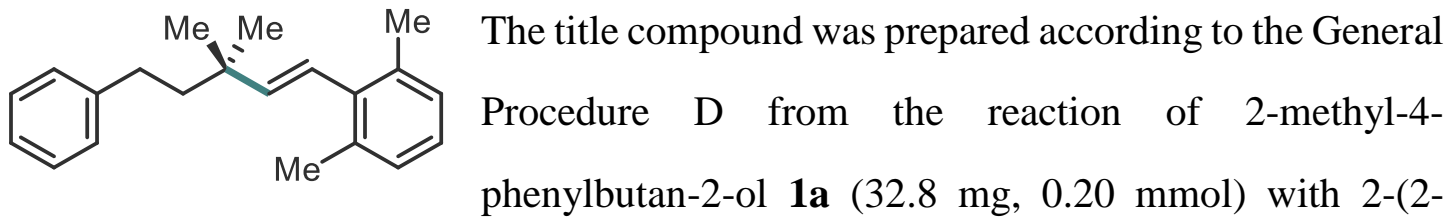
bromovinyl)-1,3-dimethylbenzene $2 \mathrm{~h}(\mathrm{E} / \mathrm{Z}=9: 1,75.6,0.36 \mathrm{mmol})$.

$42.8 \mathrm{mg}, 77 \%$ yield, colorless oil.

${ }^{1}$ H NMR $(600$ MHz, CDCl 3$) \delta 7.31(\mathrm{~d}, J=7.2 \mathrm{~Hz}, 1 \mathrm{H}), 7.26(\mathrm{t}, J=7.8 \mathrm{~Hz}, 2 \mathrm{H}), 7.17-$ $7.15(\mathrm{~m}, 3 \mathrm{H}), 6.97(\mathrm{~d}, J=9.6 \mathrm{~Hz}, 2 \mathrm{H}), 6.50(\mathrm{~d}, J=16.0 \mathrm{~Hz}, 1 \mathrm{H}), 6.04(\mathrm{~d}, J=16.0 \mathrm{~Hz}$, $1 \mathrm{H}), 2.59-2.56$ (m, $2 \mathrm{H}), 2.32$ (s, $3 \mathrm{H}), 2.30$ (s, $3 \mathrm{H}), 1.71-1.68(\mathrm{~m}, 2 \mathrm{H}), 1.17$ (s, $6 \mathrm{H})$. ${ }^{13}$ C NMR (150 MHz, CDCl 3$) \delta 143.2,140.9,136.4,134.9,134.4,130.9,128.3(2 \mathrm{C})$, $126.7,125.5,125.4,124.0,45.4,36.6,31.4,27.4,21.0,19.8$.

IR (neat, cm$^{-1}$ ): 3026, 2959, 2922, 2863, 1560, 1496, 1455, 1382, 1031, 935, 804, 741, 698.

HRMS (EI): $[\mathrm{M}]^{+}$calcd for $\mathrm{C}_{21} \mathrm{H}_{26} 278.2035$, found 278.2039 . 


\section{(E)-1,3-di-tert-butyl-5-(3,3-dimethyl-5-phenylpent-1-en-1-yl)benzene (3z)}<smiles>CC(C)(/C=C/c1cc(C(C)(C)C)cc(C(C)(C)C)c1)CCc1ccccc1</smiles>

The title compound was prepared according to the General Procedure D from the reaction of 2-methyl-4phenylbutan-2-ol 1a $(32.8 \mathrm{mg}, 0.20 \mathrm{mmol})$ with 1-(2bromovinyl)-3,5-di-tert-butylbenzene $2 \mathbf{i}(\mathrm{E} / \mathrm{Z}=18: 1,105.9,0.36 \mathrm{mmol})$. $60.1 \mathrm{mg}, 83 \%$ yield, colorless oil.

${ }^{1}$ H NMR (400 MHz, CDCl $) \delta$ 7.30-7.24 (m, 5 H), 7.21-7.14 (m, 3 H), $6.37(\mathrm{~d}, J=$ $16.0 \mathrm{~Hz}, 1 \mathrm{H}), 6.21$ (d, $J=16.0 \mathrm{~Hz}, 1 \mathrm{H}), 2.61-2.56$ (m, $2 \mathrm{H}), 1.73-1.69$ (m, $2 \mathrm{H}), 1.34$ (s, $18 \mathrm{H}), 1.18(\mathrm{~s}, 6 \mathrm{H})$.

${ }^{13}$ C NMR (100 MHz, CDCl 3$) \delta 150.9,143.3,139.3,137.0,128.3$ (2C), 127.2, 125.5, $121.3,120.3,45.3,36.5,34.8,31.5,31.3,27.4$.

IR (neat, cm$^{-1}$ ): 3026, 2962, 2906, 2866, 1649, 1427, 1248, 1202, 970, 923, 876, 764, $743,699$.

HRMS (EI): $[\mathrm{M}]^{+}$calcd for $\mathrm{C}_{27} \mathrm{H}_{38} 362.2974$, found 362.2963.

(E)-(4-(3,3-dimethyl-5-phenylpent-1-en-1-yl)phenyl)(methyl)sulfane (3aa)

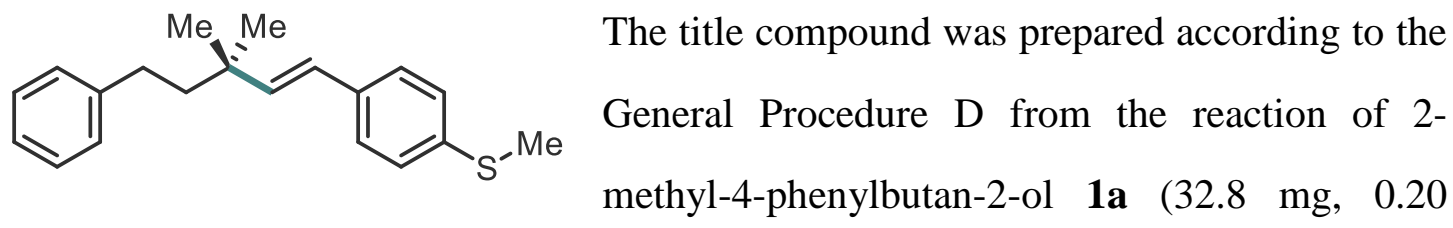
mmol) with 4-(2-bromovinyl)phenyl(methyl)sulfane 2j (E/Z = 0.9 : 1, $82.1 \mathrm{mg}, 0.36$ mmol).

$30.8 \mathrm{mg}, 52 \%$ yield, colorless oil.

${ }^{1}$ H NMR (400 MHz, CDCl 3$) \delta 7.30(\mathrm{~d}, J=8.4 \mathrm{~Hz}, 2 \mathrm{H}), 7.25(\mathrm{~d}, J=6.8 \mathrm{~Hz}, 2 \mathrm{H}), 7.21$ $(\mathrm{d}, J=8.4 \mathrm{~Hz}, 2 \mathrm{H}), 7.17-7.14(\mathrm{~m}, 3 \mathrm{H}), 6.29(\mathrm{~d}, J=16.4 \mathrm{~Hz}, 1 \mathrm{H}), 6.19$ (d, $J=16.4$ Hz, 1 H), 2.58-2.54 (m, 2 H), 2.48 (s, 3 H), 1.71-1.67 (m, 2 H), 1.16 (s, 6 H).

${ }^{13}$ C NMR (100 MHz, $\left.\mathbf{C D C l}_{3}\right) \delta 143.1,139.8,136.6,135.1,128.3,128.2,127.0,126.5$, $125.6(2 \mathrm{C}), 45.3,36.5,31.3,27.2,16.1$.

IR (neat, $\mathbf{c m}^{-1}$ ): 3025, 2958, 2922, 2862, 1603, 1545, 1263, 1157, 1072, 971, 856, 765, 699.

HRMS (ESI): $[\mathrm{M}+\mathrm{H}]^{+}$calcd for $\mathrm{C}_{20} \mathrm{H}_{25} \mathrm{~S} 297.1671$, found 297.1674. 


\section{Methyl (E)-3-(3,3-dimethyl-5-phenylpent-1-en-1-yl)benzoate (3ab)}<smiles>COC(=O)c1cccc(/C=C/C(C)(C)CCc2ccccc2)c1</smiles>

The title compound was prepared according to the General Procedure D from the reaction of 2-methyl-4phenylbutan-2-ol 1a (32.8 $\mathrm{mg}, 0.20 \mathrm{mmol})$ with methyl-3-(2-bromovinyl)benzoate $2 \mathbf{k}((\mathrm{E} / \mathrm{Z}=9: 1$,

$86.4 \mathrm{mg}, 0.36 \mathrm{mmol})$.

$43.1 \mathrm{mg}, 70 \%$ yield, colorless oil.

${ }^{1}$ H NMR (400 MHz, CDCl $) \delta 8.06(\mathrm{~s}, 1 \mathrm{H}), 7.87(\mathrm{~d}, J=8.0 \mathrm{~Hz}, 1 \mathrm{H}), 7.54(\mathrm{~d}, J=7.6$ $\mathrm{Hz}, 1 \mathrm{H}), 7.37$ (t, $J=7.6 \mathrm{~Hz}, 1 \mathrm{H}), 7.28-7.24(\mathrm{~m}, 2 \mathrm{H}), 7.18-7.14(\mathrm{~m}, 3 \mathrm{H}), 6.38(\mathrm{~d}, J=$ $16.4 \mathrm{~Hz}, 1 \mathrm{H}), 6.31$ (d, $J=16.4 \mathrm{~Hz}, 1 \mathrm{H}), 3.93$ (s, $3 \mathrm{H}), 2.59-2.55$ (m, $2 \mathrm{H}), 1.73-1.60$ (m, $2 \mathrm{H}), 1.18$ (s, $6 \mathrm{H})$.

${ }^{13}$ C NMR (100 MHz, CDCl3) $\delta$ 167.2, 143.0, 141.5, 138.2, 130.5, 130.4, 128.5, 128.3, $128.2,127.8,127.0,125.6,125.4,52.1,45.1,36.6,31.3,27.1$.

IR (neat, cm$^{-1}$ ): 3062, 3027, 2955, 2864, 1724, 1439, 1292, 1023, 1107, 971, 915, 750, 699.

HRMS (ESI): $[\mathrm{M}+\mathrm{H}]^{+}$calcd for $\mathrm{C}_{21} \mathrm{H}_{25} \mathrm{O}_{2} 309.1849$, found 309.1843 .

\section{Methyl (E)-2-(3,3-dimethyl-5-phenylpent-1-en-1-yl)benzoate (3ac)}<smiles>COC(=O)c1ccccc1/C=C/C(C)(C)CCc1ccccc1</smiles>

The title compound was prepared according to the General Procedure D from the reaction of 2-methyl-4phenylbutan-2-ol 1a(32.8 mg, $0.20 \mathrm{mmol})$ with methyl-2-

(2-bromovinyl)benzoate $2 \mathbf{l}((\mathrm{E} / \mathrm{Z}=11: 1,86.4 \mathrm{mg}, 0.36 \mathrm{mmol})$.

$47.5 \mathrm{mg}, 77 \%$ yield, colorless oil.

${ }^{1}$ H NMR (400 MHz, CDCl 3$) \delta 7.87(\mathrm{~d}, J=8.0 \mathrm{~Hz}, 1 \mathrm{H}), 7.52(\mathrm{~d}, J=7.2 \mathrm{~Hz}, 1 \mathrm{H}), 7.47-$ 7.43 (m, 1 H), 7.28-7.24 (m, 4 H), 7.19-7.16 (m, 2 H), 7.13 (d, J = 16.4 Hz, 1 H), 6.09 $(\mathrm{d}, J=16.4 \mathrm{~Hz}, 1 \mathrm{H}), 3.89$ (s, $3 \mathrm{H}), 2.63-2.59(\mathrm{~m}, 2 \mathrm{H}), 1.74-1.69(\mathrm{~m}, 2 \mathrm{H}), 1.20(\mathrm{~s}, 6$ $\mathrm{H})$.

${ }^{13}$ C NMR (100 MHz, CDCl 3$) \delta 168.0,143.2,142.8,140.0,132.0,130.4,128.3$ (2C), $128.2,127.3,126.5,125.5(2 \mathrm{C}), 52.0,45.2,36.8,31.3,27.2$.

IR (neat, $\mathbf{c m}^{-1}$ ): 3063, 3026, 2954, 2860, 1722, 1640, 1568, 1495, 1470, 1453, 1385, 
$1251,1078,1048,971,747,700$.

HRMS (ESI): $[\mathrm{M}+\mathrm{H}]^{+}$calcd for $\mathrm{C}_{21} \mathrm{H}_{25} \mathrm{O}_{2} 309.1849$, found 309.1842 .

(E)-1-(3,3-dimethyl-5-phenylpent-1-en-1-yl)-4-(trifluoromethyl)benzene (3ad, known compound)<smiles>CC(C)(C=Cc1ccc(C(F)(F)F)cc1)CCc1ccccc1</smiles>
The title compound was prepared according to the General Procedure D from the reaction of 2-methyl4-phenylbutan-2-ol 1a (32.8 mg, $0.20 \mathrm{mmol})$ with (E)-1-(2-bromovinyl)-4-(trifluoromethyl)benzene $2 \mathrm{~m}(90.0 \mathrm{mg}, 0.36 \mathrm{mmol}) . .{ }^{1} \mathrm{H}$ NMR and ${ }^{13} \mathrm{C}$ NMR data are consistent with those reported in reference 7.

$49.6 \mathrm{mg}, 78 \%$ yield, colorless oil.

${ }^{1}$ H NMR (400 MHz, CDCl3) $\delta 7.54(\mathrm{~d}, J=8.0 \mathrm{~Hz}, 2 \mathrm{H}), 7.44(\mathrm{~d}, J=8.0 \mathrm{~Hz}, 2 \mathrm{H})$, 7.28-7.23 (m, 2 H), 7.17-7.15 (m, 3 H), 6.37 (d, $J=16.4 \mathrm{~Hz}, 1 \mathrm{H}), 6.32(\mathrm{~d}, J=16.4 \mathrm{~Hz}$, $1 \mathrm{H}), 2.59-2.55$ (m, $2 \mathrm{H}), 1.74-1.70$ (m, $2 \mathrm{H}), 1.18$ (s, $6 \mathrm{H})$.

${ }^{13}$ C NMR (100 MHz, CDCl $) \delta$ 143.0, 142.9, 141.5, 128.7 (q, $J=32$ Hz), 128.4, 128.3, 126.2, 125.7, $125.4(\mathrm{q}, J=4 \mathrm{~Hz}), 125.2,123.0,45.1,36.7,31.3,27.1$.

${ }^{19}$ F NMR (376 MHz, CDCl 3$): ~ \delta-62.4$.

IR (neat, cm$^{-1}$ ): 3063, 3027, 2962, 2865, 1616, 1495, 1455, 1385, 1326, 1165, 1109, 1068, 1016, 972, 900, 866, 757, 699.

(E)-4-(3,3-dimethyl-5-phenylpent-1-en-1-yl)-1,2-difluorobenzene (3ae)<smiles>CC(C)(/C=C/c1ccc(F)c(F)c1)CCc1ccccc1</smiles>

The title compound was prepared according to the General Procedure D from the reaction of 2-methyl-4phenylbutan-2-ol 1a $(32.8 \mathrm{mg}, 0.20 \mathrm{mmol})$ with 4-(2bromovinyl)-1,2-difluorobenzene $2 n(E / Z=13: 1$,

$78.5 \mathrm{mg}, 0.36 \mathrm{mmol})$.

$48.6 \mathrm{mg}, 85 \%$ yield, colorless oil.

${ }^{1}$ H NMR (400 MHz, CDCl3) $\delta$ 7.28-7.25 (m, 2 H), 7.19-7.14 (m, 4 H), 7.11-7.01 (m, $2 \mathrm{H}), 6.24(\mathrm{~d}, J=16.4 \mathrm{~Hz}, 1 \mathrm{H}), 6.13(\mathrm{~d}, J=16.4 \mathrm{~Hz}, 1 \mathrm{H}), 2.58-2.53(\mathrm{~m}, 2 \mathrm{H}), 1.72-$ $1.67(\mathrm{~m}, 2 \mathrm{H}) 1.16(\mathrm{~s}, 6 \mathrm{H})$.

${ }^{13}$ C NMR (100 MHz, CDCl3) $\delta 151.7(\mathrm{~d}, J=12.0 \mathrm{~Hz}), 150.6(\mathrm{~d}, J=13.0 \mathrm{~Hz}), 149.2$ 
(d, $J=13.0 \mathrm{~Hz}), 148.1(\mathrm{~d}, J=13.0 \mathrm{~Hz}), 142.9,141.4(\mathrm{~d}, J=2.0 \mathrm{~Hz}), 135.2(\mathrm{q}, J=4.0$ $\mathrm{Hz}), 128.3(\mathrm{~d}, J=7.0 \mathrm{~Hz}), 125.7,124.4,122.1(\mathrm{q}, J=4.0 \mathrm{~Hz}), 117.1(\mathrm{~d}, J=17.0 \mathrm{~Hz})$, $114.3(\mathrm{~d}, J=18.0 \mathrm{~Hz}), 45.1,36.5,31.3,27.1$.

${ }^{19}$ F NMR (376 MHz, CDCl3): $\delta$-138.3, -138.4, -140.4, -140.5.

IR (neat, cm$^{-1}$ ): 3063, 3028, 2961, 2864, 1650, 1517, 1293, 1274, 1211, 1113, 970, 808, $777,699$.

HRMS (ESI): $[\mathrm{M}+\mathrm{H}]^{+}$calcd for $\mathrm{C}_{19} \mathrm{H}_{21} \mathrm{~F}_{2} 287.1606$, found 287.1605.

(E)-4-(3,3-dimethylbut-1-en-1-yl)benzonitrile (3af)

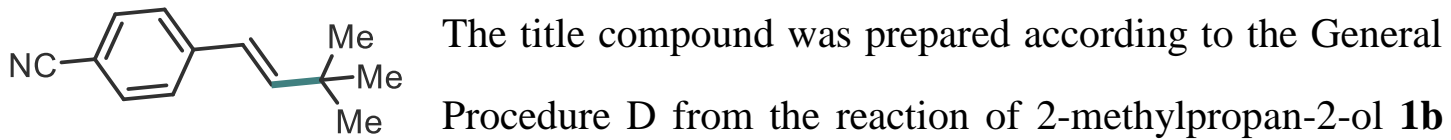
(29.2 mg, $0.40 \mathrm{mmol}$ ) with 4-(2-bromovinyl)benzonitrile 2o (E/Z = 9: 1, $41.4 \mathrm{mg}, 0.20$ mmol).

$24.4 \mathrm{mg}, 66 \%$ yield, colorless oil.

${ }^{1}$ H NMR (400 MHz, CDCl 3$) \delta 7.57(\mathrm{~d}, J=8.0 \mathrm{~Hz}, 2 \mathrm{H}), 7.43(\mathrm{~d}, J=8.0 \mathrm{~Hz}, 2 \mathrm{H})$, $6.40(\mathrm{~d}, J=16.0 \mathrm{~Hz}, 1 \mathrm{H}), 6.30(\mathrm{~d}, J=16.0 \mathrm{~Hz}, 1 \mathrm{H}), 1.13(\mathrm{~s}, 9 \mathrm{H})$.

${ }^{13}$ C NMR (100 MHz, CDCl 3$) \delta 145.9,142.6,132.3,126.5,123.4,119.2,109.9,33.7$, 29.3 .

IR (neat, $\mathbf{c m}^{-1}$ ): 3035, 2961, 2905, 2867, 2226, 1646, 1605, 1504, 1475, 1391, 1364 , $1205,1108,972,867,816$.

HRMS (ESI): $[\mathrm{M}+\mathrm{Na}]^{+}$calcd for $\mathrm{C}_{13} \mathrm{H}_{15} \mathrm{NNa} 208.1097$, found 208.1098.

(E)-2-(4-(3,3-dimethyl-5-phenylpent-1-en-1-yl)phenyl)-4,4,5,5-tetramethyl-1,3,2dioxaborolane (3ag)<smiles>CC(C)(C=Cc1ccc(Br)cc1)CCc1ccccc1</smiles>
The title compound was prepared according to the General Procedure D from the reaction of 2-methyl4-phenylbutan-2-ol 1a (32.8 mg, $0.20 \mathrm{mmol})$ with (E)-2-(4-(2-bromovinyl)phenyl)-4,4,5,5-tetramethyl-1,3,2-dioxaborolane $\quad \mathbf{2 p} \quad$ (110.9 $\mathrm{mg}, 0.36 \mathrm{mmol})$.

$62.8 \mathrm{mg}, 83 \%$ yield, colorless oil.

${ }^{1}$ H NMR (400 MHz, CDCl3) $\delta 7.75(\mathrm{~d}, J=7.6 \mathrm{~Hz}, 2 \mathrm{H}), 7.37(\mathrm{~d}, J=7.6 \mathrm{~Hz}, 2 \mathrm{H})$, 
7.28-7.23 (m, 2 H), 7.17-7.14 (m, 3 H), 6.36 (d, J=16.4 Hz, 1 H), 6.29 (d, J=16.4 Hz, $1 \mathrm{H}), 2.58-2.54$ (m, $2 \mathrm{H}), 1.72-1.68$ (m, $2 \mathrm{H}), 1.34(\mathrm{~s}, 12 \mathrm{H}), 1.17$ (s, $6 \mathrm{H})$.

${ }^{13}$ C NMR (100 MHz, CDCl3) $\delta 143.1,141.3,140.7,135.0,128.3,128.2,126.3,125.6$, $125.4(2 \mathrm{C}), 83.7,45.2,36.6,31.3,27.1,24.8$.

IR (neat, $\mathbf{c m}^{-1}$ ): 3027, 2976, 2931, 2865, 1645, 1456, 1361, 1291, 1213, 1145, 1019 , $963,861,765,740$.

HRMS (ESI): [M+Na $]^{+}$calcd for $\mathrm{C}_{25} \mathrm{H}_{33} \mathrm{BNaO}_{2} 399.2466$, found 399.2471.

(E)-2-(3,3-dimethylbut-1-en-1-yl)furan (3ah, known compound)

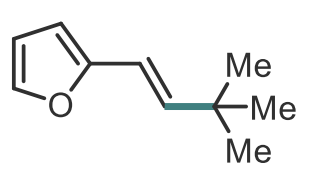

The title compound was prepared according to the General Procedure D from the reaction of 2-methylpropan-2-ol 1b $(29.2 \mathrm{mg}$ $0.20 \mathrm{mmol})$ with 2-(2-bromovinyl)furan $\mathbf{2 q}(\mathrm{E} / \mathrm{Z}=2: 1,34.4 \mathrm{mg}$, $0.20 \mathrm{mmol}) .{ }^{1} \mathrm{H} \mathrm{NMR}$ and ${ }^{13} \mathrm{C} \mathrm{NMR}$ data are consistent with those reported in reference 22.

$18.6 \mathrm{mg}, 62 \%$ yield, colorless oil.

${ }^{1} \mathbf{H}$ NMR $(400 \mathrm{MHz}, \mathbf{C D C l}) \delta 7.31(\mathrm{~d}, J=1.6 \mathrm{~Hz}, 1 \mathrm{H}), 6.34\left(\mathrm{dd}, J_{1}=2 \mathrm{~Hz}, J_{2}=3.2\right.$ $\mathrm{Hz}, 1 \mathrm{H}), 6.23$ (d, $J=16.0 \mathrm{~Hz}, 1 \mathrm{H}), 6.15-6.11(\mathrm{~m}, 2 \mathrm{H}), 1.10(\mathrm{~s}, 9 \mathrm{H})$.

${ }^{13}$ C NMR (100 MHz, CDCl 3$) \delta 153.5,141.1,140.8,113.7,111.1,106.1,33.2,29.5$.

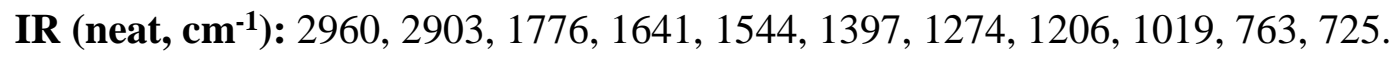

\section{(E)-2-(3,3-dimethylbut-1-en-1-yl)thiophene (3ai, known compound)}

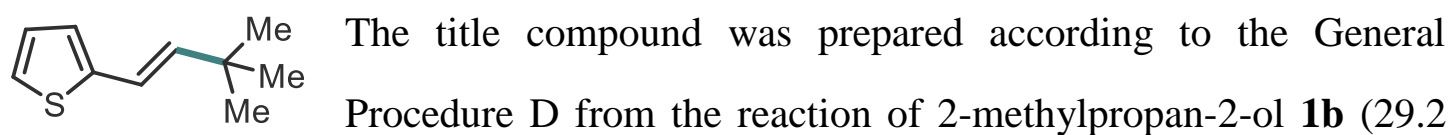
$\mathrm{mg}, 0.40 \mathrm{mmol})$ with 2-(2-bromovinyl)thiophene $2 \mathbf{r}(\mathrm{E} / \mathrm{Z}=2.5: 1,37.6 \mathrm{mg}, 0.20 \mathrm{mmol})$. ${ }^{1} \mathrm{H}$ NMR and ${ }^{13} \mathrm{C}$ NMR data are consistent with those reported in reference 23.

$19.3 \mathrm{mg}, 58 \%$ yield, colorless oil.

${ }^{1} \mathbf{H}$ NMR (400 MHz, CDCl 3$) \delta 7.08(\mathrm{~d}, J=5.2 \mathrm{~Hz}, 1 \mathrm{H}), 6.93\left(\mathrm{dd}, J_{l}=3.6 \mathrm{~Hz}, J_{2}=5.2\right.$ $\mathrm{Hz}, 1 \mathrm{H}), 6.88(\mathrm{~d}, J=3.6 \mathrm{~Hz}, 1 \mathrm{H}), 6.43(\mathrm{~d}, J=16.0 \mathrm{~Hz}, 1 \mathrm{H}), 6.09$ (d, $J=16.0 \mathrm{~Hz}, 1$ $\mathrm{H}), 1.10(\mathrm{~s}, 9 \mathrm{H})$.

${ }^{13}$ C NMR (100 MHz, CDCl 3$) \delta 143.4,141.8,127.2,124.3,123.0,118.2,33.4,29.5$.

IR (neat, cm$^{-1}$ ): 2960, 2903, 1776, 1545, 1461, 1226, 1223, 1078, 958, 854, 808, 691. 


\section{(E)-(3,3-dimethylhex-4-ene-1,5-diyl)dibenzene (3aj)}

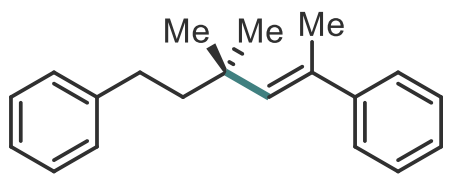

The title compound was prepared according to the General

Procedure D from the reaction of 2-methyl-4phenylbutan-2-ol 1a $(32.8 \mathrm{mg}, 0.20 \mathrm{mmol})$ with (E)-(1bromoprop-1-en-2-yl)benzene $2 \mathrm{~s}$ (70.6 mg, $0.36 \mathrm{mmol}$ ).

$26.4 \mathrm{mg}, 50 \%$ yield, colorless oil.

${ }^{1} \mathbf{H}$ NMR (400 MHz, CDCl 3$) \delta$ 7.37-7.35 (m, 2 H), 7.32-7.26 (m, 4 H), 7.24-7.15 (m, $4 \mathrm{H}), 5.70(\mathrm{~d}, J=1.2 \mathrm{~Hz}, 1 \mathrm{H}), 2.66-2.62(\mathrm{~m}, 2 \mathrm{H}), 2.17(\mathrm{~d}, J=1.2 \mathrm{~Hz}, 3 \mathrm{H}), 1.82-1.78$ (m, $2 \mathrm{H}), 1.27$ (s, $6 \mathrm{H})$.

${ }^{13}$ C NMR (100 MHz, CDCl3) $\delta$ 146.2, 143.4, 137.7, 135.2, 128.3, 128.2, 128.1, 126.4, 126.0, 125.6, 46.1, 36.1, 31.7, 29.1, 17.4.

IR (neat, $\mathbf{c m}^{-1}$ ): 2959, 2863, 1639, 1493, 1454, 1364, 1073, 1029, 797, 698.

HRMS (ESI): $[\mathrm{M}+\mathrm{H}]^{+}$calcd for $\mathrm{C}_{20} \mathrm{H}_{25}$ 265.1951, found 265.1954 .

((1E,3E)-5,5-dimethylhepta-1,3-diene-1,7-diyl)dibenzene (3ak)

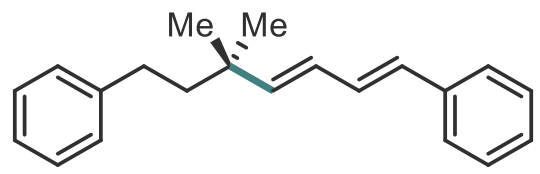

The title compound was prepared according to the General Procedure D from the reaction of 2-methyl4-phenylbutan-2-ol 1a $(32.8 \mathrm{mg}, 0.20 \mathrm{mmol})$ with ((1E)-4-bromobuta-1,3-dien-1-yl)benzene $2 \mathrm{t}(\mathrm{E} / \mathrm{Z}=4.5: 1,74.9 \mathrm{mg}, 0.36 \mathrm{mmol})$. $34.2 \mathrm{mg}, 62 \%$ yield, colorless oil.

${ }^{1}$ H NMR (400 MHz, CDCl 3$) \delta 7.39(\mathrm{~d}, J=7.6 \mathrm{~Hz}, 2 \mathrm{H})$, 7.32-7.24 (m, $\left.4 \mathrm{H}\right)$, 7.21-7.14 $(\mathrm{m}, 4 \mathrm{H}), 6.79\left(\mathrm{dd}, J_{l}=10.4 \mathrm{~Hz}, J_{2}=15.6 \mathrm{~Hz}, 1 \mathrm{H}\right), 6.50(\mathrm{~d}, J=15.6 \mathrm{~Hz}, 1 \mathrm{H}), 6.19$ $\left(\mathrm{dd}, J_{1}=10.4 \mathrm{~Hz}, J_{2}=15.6 \mathrm{~Hz}, 1 \mathrm{H}\right), 5.84(\mathrm{~d}, J=15.6 \mathrm{~Hz}, 1 \mathrm{H}), 2.57-2.52(\mathrm{~m}, 2 \mathrm{H})$, $1.67-1.51(\mathrm{~m}, 2 \mathrm{H}), 1.12(\mathrm{~s}, 6 \mathrm{H})$.

${ }^{13}$ C NMR (100 MHz, CDCl 3$) \delta 145.0,143.2,137.7,130.3,129.8,128.6,128.3,128.2$, 127.1, 127.0, 126.1, 125.6, 45.3, 36.5, 31.3, 27.2.

IR (neat, $\mathbf{c m}^{-1}$ ): 3082, 3061, 2960, 2863, 1640, 1599, 1543, 1494, 1156, 1072, 746, 695.

HRMS (ESI): $[\mathrm{M}+\mathrm{H}]^{+}$calcd for $\mathrm{C}_{21} \mathrm{H}_{25} 277.1951$, found 277.1946. 
((1E,3E)-3,5,5-trimethylhepta-1,3-diene-1,7-diyl)dibenzene (3al)

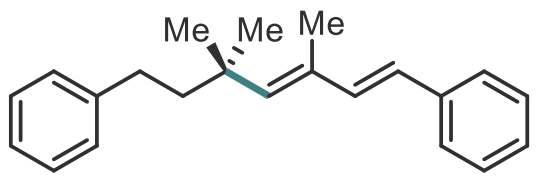

The title compound was prepared according to the General Procedure D from the reaction of 2-methyl4-phenylbutan-2-ol 1a (32.8 mg, $0.20 \mathrm{mmol})$ with ((1E)-4-bromo-3-methylbuta-1,3-dien-1-yl)benzene 2u (E/Z = $3: 1,79.9 \mathrm{mg}, 0.36$ mmol).

$30.8 \mathrm{mg}, 53 \%$ yield, colorless oil.

${ }^{1}$ H NMR (400 MHz, CDCl 3$) \delta 7.41(\mathrm{~d}, J=8.0 \mathrm{~Hz}, 2 \mathrm{H})$, 7.32-7.23 (m, 4 H), 7.20-7.15 (m, $4 \mathrm{H}), 6.79(\mathrm{~d}, J=16.0 \mathrm{~Hz}, 1 \mathrm{H}), 6.47(\mathrm{~d}, J=16.0 \mathrm{~Hz}, 1 \mathrm{H}), 5.66(\mathrm{~s}, 1 \mathrm{H}), 2.61-2.57$ (m, $2 \mathrm{H}), 2.00(\mathrm{~s}, 3 \mathrm{H}), 1.80-1.76(\mathrm{~m}, 2 \mathrm{H}), 1.24(\mathrm{~s}, 6 \mathrm{H})$.

${ }^{13}$ C NMR (100 MHz, CDCl3) $\delta$ 143.2, 143.1, 137.9, 135.9, 133.6, 128.5, 128.3 (2C), $126.8,126.2,125.6,125.1,46.2,36.2,31.6,29.2,13.5$.

IR (neat, cm$^{-1}$ ): 3081, 3061, 3026, 2958, 2864, 1800, 1600, 1494, 1451, 1384, 1092 , $746,695$.

HRMS (ESI): $[\mathrm{M}+\mathrm{H}]^{+}$calcd for $\mathrm{C}_{22} \mathrm{H}_{27}$ 291.2107, found 291.2105.

((1E,3E)-2,5,5-trimethylhepta-1,3-diene-1,7-diyl)dibenzene (3am)<smiles>CC(C=CC(C)(C)CCc1ccccc1)=Cc1ccccc1</smiles>
The title compound was prepared according to the General Procedure D from the reaction of 2-methyl4-phenylbutan-2-ol 1a $(32.8 \mathrm{mg}, 0.20 \mathrm{mmol})$ with ((1E,3E)-4-bromo-2-methylbuta-1,3-dien-1-yl)benzene 2v (79.9 mg, $0.36 \mathrm{mmol}$ ). $25.0 \mathrm{mg}, 43 \%$ yield (4:1 mixture of stereoisomers), colorless oil.

${ }^{1}$ H NMR (400 MHz, CDCl 3$) \delta$ 7.35-7.26 (m, 6 H), 7.24-7.13 (m, 4 H), 6.62 (d, $J=$ $16.0 \mathrm{~Hz}, 0.18 \mathrm{H}),[6.49(\mathrm{~s}, 0.82 \mathrm{H}), 6.39(\mathrm{~s}, 0.21 \mathrm{H})], 6.21(\mathrm{~d}, J=16.0 \mathrm{~Hz}, 0.82 \mathrm{H})$, [5.84 (d, $J=16.0 \mathrm{~Hz}, 0.2 \mathrm{H}), 5.78(\mathrm{~d}, J=16.0 \mathrm{~Hz}, 0.8 \mathrm{H})], 2.58-2.54(\mathrm{~m}, 2 \mathrm{H}),[2.01$ $(\mathrm{d}, J=1.2 \mathrm{~Hz}, 0.63 \mathrm{H}), 2.00(\mathrm{~d}, J=1.2 \mathrm{~Hz}, 2.35 \mathrm{H})], 1.69-1.65(\mathrm{~m}, 2 \mathrm{H}),[1.14(\mathrm{~s}, 4.8$ H), 1.08 (s, $1.2 \mathrm{H})]$.

${ }^{13}$ C NMR (100 MHz, CDCl3) $\delta$ 143.2, 141.8, 139.5, 138.1, 135.9, 131.5, 129.6, 129.3, $129.1,128.3,128.1,127.9,127.8,126.2,125.5,124.8,45.4,45.3,36.5,36.3,31.3,27.4$, 14.0 
IR (neat, cm$^{-1}$ ): 3061, 3025, 2959, 2862, 1637, 1494, 1453, 1074, 1030, 746, 698.

HRMS (ESI): $[\mathrm{M}+\mathrm{H}]^{+}$calcd for $\mathrm{C}_{22} \mathrm{H}_{27} 291.2107$, found 291.2101.

((4E,6E)-3,3-dimethylocta-4,6-diene-1,7-diyl)dibenzene (3an)<smiles>CC(=CC=CC(C)(C)CCc1ccccc1)c1ccccc1</smiles>

The title compound was prepared according to the General Procedure D from the reaction of 2-methyl4-phenylbutan-2-ol 1a $(32.8 \mathrm{mg}, 0.20 \mathrm{mmol})$ with ((2E)-5-bromopenta-2,4-dien-2-yl)benzene 2w (E/Z = 1 : 1, $79.9 \mathrm{mg}, 0.36 \mathrm{mmol})$. $43.5 \mathrm{mg}, 75 \%$ yield, colorless oil.

${ }^{1}$ H NMR (400 MHz, CDCl 3$) \delta 7.45(\mathrm{~d}, J=8.0 \mathrm{~Hz}, 2 \mathrm{H})$, 7.33-7.30 (m, $\left.2 \mathrm{H}\right)$, 7.28-7.20 (m, 3 H), 7.18-7.14 (m, 3 H), 6.49-6.37 (m, 2 H), 5.84 (d, $J=14.8$ Hz, 1 H), 2.57-2.53 (m, $2 \mathrm{H}), 2.18(\mathrm{~s}, 3 \mathrm{H}), 1.68-1.63(\mathrm{~m}, 2 \mathrm{H}), 1.14(\mathrm{~s}, 6 \mathrm{H})$.

${ }^{13}$ C NMR (100 MHz, CDCl 3$) \delta$ 145.2, 143.4, 143.2, 134.0, 128.3 (2C), 128.2, 127.6, $126.7,125.6,125.5,123.4,45.3,36.7,31.4,27.3,16.0$.

IR (neat, cm$^{-1}$ ): 3083, 3059, 3027, 2959, 2863, 1687, 1494, 1448, 1387, 1363, 1308, 1073, 970, 758, 697.

HRMS (ESI): $[\mathrm{M}+\mathrm{H}]^{+}$calcd for $\mathrm{C}_{22} \mathrm{H}_{27}$ 291.2107, found 291.2101.

(E)-(3,3,7-trimethylocta-4,6-dien-1-yl)benzene (3ao)

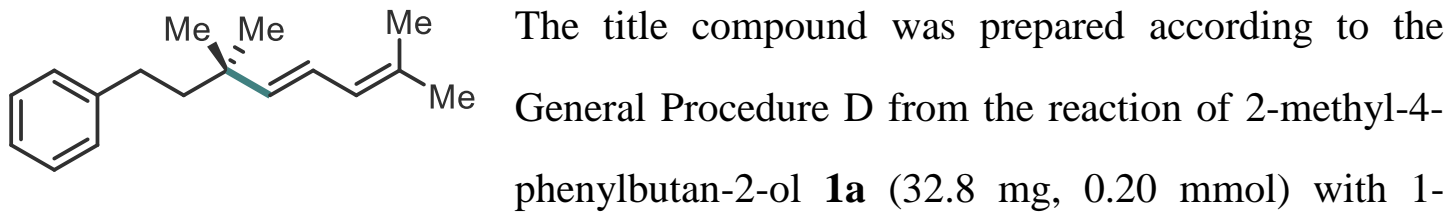
bromo-4-methylpenta-1,3-diene $2 \mathbf{x}(\mathrm{E} / \mathrm{Z}=1.6: 1,57.6 \mathrm{mg}, 0.36 \mathrm{mmol})$.

$20.6 \mathrm{mg}, 45 \%$ yield, colorless oil.

${ }^{1}$ H NMR (400 MHz, CDCl $) \delta$ 7.28-7.24 (m, 2 H), 7.17-7.14 (m, 3 H), $6.20\left(\mathrm{dd}, J_{l}=\right.$ $\left.10.8 \mathrm{~Hz}, J_{2}=15.6 \mathrm{~Hz}, 1 \mathrm{H}\right), 5.84(\mathrm{~d}, J=10.8 \mathrm{~Hz}, 1 \mathrm{H}), 5.58(\mathrm{~d}, J=15.6 \mathrm{~Hz}, 1 \mathrm{H}), 2.54-$ $2.50(\mathrm{~m}, 2 \mathrm{H}), 1.78(\mathrm{~s}, 6 \mathrm{H}), 1.63-1.58(\mathrm{~m}, 2 \mathrm{H}), 1.09$ (s, $6 \mathrm{H})$.

${ }^{13}$ C NMR (100 MHz, CDCl3) $\delta$ 143.4, 141.4, 133.1, 128.3, 128.2, 125.5, 125.4, 122.9, $45.4,36.3,31.4,27.4,26.0,18.3$.

IR (neat, cm$^{-1}$ ): 3063, 2960, 2930, 1640, 1561, 1510, 1178, 1110, 764, 742, 698, 648. 
HRMS (ESI): $[\mathrm{M}]^{+}$calcd for $\mathrm{C}_{17} \mathrm{H}_{24} 228.1878$, found 228.1875 .

((4E,6E)-3,3-dimethyldeca-4,6-dien-1-yl)benzene (3ap)

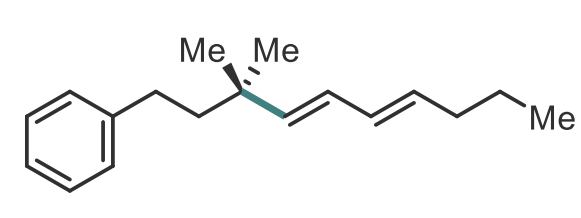

The title compound was prepared according to the

General Procedure D from the reaction of 2methyl-4-phenylbutan-2-ol $\mathbf{1 a}(32.8 \mathrm{mg}, \quad 0.20$

mmol) with (3E)-1-bromohepta-1,3-diene 2y (E/Z = 1.5 : 1, $62.6 \mathrm{mg}, 0.36 \mathrm{mmol})$.

$29.1 \mathrm{mg}, 60 \%$ yield, colorless oil.

${ }^{1} \mathbf{H}$ NMR (400 MHz, CDCl 3$) \delta$ 7.28-7.24 (m, 2 H), $7.16(\mathrm{~d}, J=6.4 \mathrm{~Hz}, 3 \mathrm{H}), 6.08-5.95$ (m, 2 H), 5.66-5.57 (m, 2 H), 2.54-2.49 (m, 2 H), 2.06 (q, J = 7.2 Hz, 2 H), 1.62-1.58 (m, $2 \mathrm{H}), 1.46-1.37$ (m, $2 \mathrm{H}), 1.07$ (s, $6 \mathrm{H}), 0.91$ (t, $J=7.2 \mathrm{~Hz}, 3 \mathrm{H})$.

${ }^{13}$ C NMR (100 MHz, CDCl3) $\delta$ 143.3, 141.6, 132.6, 130.8, 128.3, 128.2, 126.8, 125.5, $45.3,36.1,34.8,31.3,27.3,22.6,13.8$.

IR (neat, cm-1): 3063, 2960, 2930, 2871, 1694, 1542, 1496, 991, 743, 699.

HRMS (ESI): [M+H] ${ }^{+}$calcd for $\mathrm{C}_{18} \mathrm{H}_{27} 243.2107$, found 243.2104.

(E)-1-chloro-4-(3,3-dimethyl-5-phenylpent-1-en-1-yl)benzene (3aq, known compound)<smiles>CC(C)(C=Cc1ccc(Cl)cc1)CCc1ccccc1</smiles>

The title compound was prepared according to the General Procedure D from the reaction of 2-methyl-4phenylbutan-2-ol 1a (32.8 mg, $0.20 \mathrm{mmol})$ with 1-(2bromovinyl)-4-chlorobenzene $\mathbf{2 z}(\mathrm{E} / \mathrm{Z}=8: 1,77.7 \mathrm{mg}, 0.36 \mathrm{mmol}) .{ }^{1} \mathrm{H} \mathrm{NMR}$ and ${ }^{13} \mathrm{C}$ NMR data are consistent with those reported in reference 7.

$44.3 \mathrm{mg}, 78 \%$ yield, colorless oil.

${ }^{1}$ H NMR (400 MHz, CDCl3) $\delta$ 7.30-7.24 (m, 6 H), 7.17-7.14 (m, 3 H), 6.29 (d, $J=$ $16.4 \mathrm{~Hz}, 1 \mathrm{H}), 6.20$ (d, $J=16.4 \mathrm{~Hz}, 1 \mathrm{H}), 2.58-2.54$ (m, 2 H), 1.72-1.68 (m, 2 H) 1.17 (s, $6 \mathrm{H})$.

${ }^{13}$ C NMR (100 MHz, CDCl3) $\delta$ 143.0, 141.0, 136.5, 132.4, 128.6, 128.3, 128.2, 127.3, 125.6, 125.1, 45.2, 36.5, 31.3, 27.2.

IR (neat, cm-1): 3084, 2960, 2929, 2862, 1646, 1604, 1492, 1469, 1403, 1384, 1364 , 1097, 971, 807, 744, 699. 


\section{(E)-1-bromo-4-(3,3-dimethyl-5-phenylpent-1-en-1-yl)benzene (3ar)}<smiles>CC(C)(C=Cc1ccc(Br)cc1)CCc1ccccc1</smiles>

The title compound was prepared according to the General Procedure D from the reaction of 2-methyl-4phenylbutan-2-ol 1a (32.8 mg, $0.20 \mathrm{mmol})$ with 1bromo-4-(2-bromovinyl)benzene 2aa (E/Z = $8: 1,93.6 \mathrm{mg}, 0.36 \mathrm{mmol})$.

$45.9 \mathrm{mg}, 70 \%$ yield, colorless oil.

${ }^{1} \mathbf{H}$ NMR (400 MHz, CDCl 3$) \delta 7.40(\mathrm{~d}, J=8.4 \mathrm{~Hz}, 2 \mathrm{H})$, 7.27-7.20 (m, 4 H), 7.16-7.14 $(\mathrm{m}, 3 \mathrm{H}), 6.27(\mathrm{~d}, J=16.4 \mathrm{~Hz}, 1 \mathrm{H}), 6.20(\mathrm{~d}, J=16.4 \mathrm{~Hz}, 1 \mathrm{H}), 2.57-2.53(\mathrm{~m}, 2 \mathrm{H})$, 1.71-1.67 (m, $2 \mathrm{H}), 1.16$ (s, $6 \mathrm{H})$.

${ }^{13}$ C NMR (100 MHz, CDCl3) $\delta$ 143.0, 141.0, 136.9, 131.5, 128.3, 128.2, 127.6, 125.6, 125.2, 120.5, 45.1, 36.5, 31.3, 27.1.

IR (neat, $\mathbf{c m}^{-1}$ ): 3086, 3066, 3029, 2963, 2868, 1737, 1605, 1590, 1489, 1470, 1384, $1364,1100,1074,1010,971,859,699$.

HRMS (EI): [M] ${ }^{+}$calcd for $\mathrm{C}_{19} \mathrm{H}_{21} \mathrm{Br} 328.0827$, found 328.0819.

(E)-1-(3,3-dimethyl-5-phenylpent-1-en-1-yl)-4-iodobenzene (3as)<smiles>CC(C)(/C=C/c1ccc(I)cc1)CCc1ccccc1</smiles>

The title compound was prepared according to the General Procedure D from the reaction of 2-methyl-4phenylbutan-2-ol 1a (32.8 mg, $0.20 \mathrm{mmol})$ with 1-(2bromovinyl)-4-iodobenzene 2ab (E/Z = $10: 1,110.8 \mathrm{mg}, 0.36 \mathrm{mmol})$. $54.9 \mathrm{mg}, 73 \%$ yield, colorless oil.

${ }^{1}$ H NMR (400 MHz, CDCl $) \delta 7.62(\mathrm{~d}, J=8.4 \mathrm{~Hz}, 2 \mathrm{H}), 7.28-7.25(\mathrm{~m}, 2 \mathrm{H})$, 7.18-7.15 $(\mathrm{m}, 3 \mathrm{H}), 7.10(\mathrm{~d}, J=8.4 \mathrm{~Hz}, 2 \mathrm{H}), 6.26(\mathrm{~d}, J=16.4 \mathrm{~Hz}, 1 \mathrm{H}), 6.22(\mathrm{~d}, J=16.4 \mathrm{~Hz}, 1$ H), 2.57-2.53 (m, 2 H), 1.71-1.67 (m, $2 \mathrm{H}), 1.16$ (s, $6 \mathrm{H})$.

${ }^{13}$ C NMR (100 MHz, CDCl 3$) \delta 143.0,141.2,137.5,137.4,128.3,128.2,127.9,125.6$, 125.2, 91.8, 45.1, 36.6, 31.3, 27.1.

IR (neat, cm-1): 3025, 2956, 2923, 2866, 1605, 1485, 1396, 1384, 1060, 1031, 971, $859,801,742$.

HRMS (EI): [M] ${ }^{+}$calcd for $\mathrm{C}_{19} \mathrm{H}_{21} \mathrm{I} 376.0688$, found 376.0689 . 


\section{(E)-4-(3,3-dimethylbut-1-en-1-yl)phenyl methanesulfonate (3at)}

The title compound was prepared according to the General
$(29.2 \mathrm{mg}, \quad 0.40 \mathrm{mmol})$ with 4-(2-bromovinyl)phenyl methanesulfonate 2ac (E/Z $=10: 1,55.2 \mathrm{mg}, 0.20 \mathrm{mmol})$.

$36.1 \mathrm{mg}, 71 \%$ yield, colorless oil.

${ }^{1} \mathbf{H}$ NMR (600 MHz, CDCl 3$) \delta 7.38(\mathrm{~d}, J=8.4 \mathrm{~Hz}, 2 \mathrm{H}), 7.21(\mathrm{~d}, J=8.4 \mathrm{~Hz}, 2 \mathrm{H}), 6.29$ $(\mathrm{d}, J=16.2 \mathrm{~Hz}, 1 \mathrm{H}), 6.24(\mathrm{~d}, J=16.2 \mathrm{~Hz}, 1 \mathrm{H}), 3.11(\mathrm{~s}, 3 \mathrm{H}), 1.12(\mathrm{~s}, 9 \mathrm{H})$.

${ }^{13}$ C NMR (150 MHz, CDCl $) \delta 147.9,143.3,137.5,127.3,123.2,122.0,37.1,33.4$, 29.4 .

IR (neat, $\mathbf{c m}^{-1}$ ): 3038, 2961, 2868, 1646, 1504, 1478, 1416, 1370, 1200, 1176, 1151, 1103, 971, 844, 695 .

HRMS (ESI): [M+Na] ${ }^{+}$calcd for $\mathrm{C}_{13} \mathrm{H}_{18} \mathrm{NaO}_{3} \mathrm{~S} 277.0869$, found 277.0868 .

\section{(E)-4-(3,3-dimethyl-5-phenylpent-1-en-1-yl)phenyl pivalate (3au)}

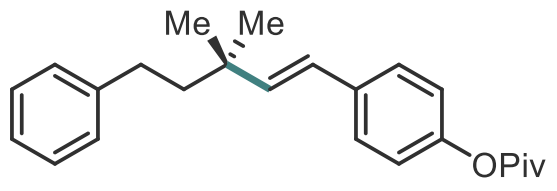

The title compound was prepared according to the General Procedure D from the reaction of 2-methyl4-phenylbutan-2-ol 1a $(32.8 \mathrm{mg}, 0.20 \mathrm{mmol})$ with 4-(2-bromovinyl)phenyl pivalate 2ad (E/Z = $11: 1,101.5 \mathrm{mg}, 0.36 \mathrm{mmol})$. $45.5 \mathrm{mg}, 65 \%$ yield, colorless oil.

${ }^{1} \mathbf{H}$ NMR (600 MHz, CDCl 3$) \delta 7.36(\mathrm{~d}, J=8.4 \mathrm{~Hz}, 2 \mathrm{H})$, 7.27-7.24 (m, $\left.2 \mathrm{H}\right)$, 7.17-7.15 $(\mathrm{m}, 3 \mathrm{H}), 6.99(\mathrm{~d}, J=8.4 \mathrm{~Hz}, 2 \mathrm{H}), 6.32(\mathrm{~d}, J=16.2 \mathrm{~Hz}, 1 \mathrm{H}), 6.18(\mathrm{~d}, J=16.2 \mathrm{~Hz}, 1$ H), 2.58-2.55 (m, $2 \mathrm{H}), 1.71-1.68(\mathrm{~m}, 2 \mathrm{H}), 1.35(\mathrm{~s}, 9 \mathrm{H}), 1.17(\mathrm{~s}, 6 \mathrm{H})$.

${ }^{13}$ C NMR (150 MHz, CDCl 3$) \delta 177.1,150.0,143.1,140.3,135.5,128.3,128.2,126.9$, $125.6,125.4,121.5,45.3,39.1,36.5,31.3,27.2,27.1$.

IR (neat, cm$^{-1}$ ): 3029, 2963, 2865, 1752, 1605, 1508, 1480, 1457, 1396, 1278, 1200 , 1167, 1116, 1031, 971, 742, 699.

HRMS (ESI): [M+Na] ${ }^{+}$calcd for $\mathrm{C}_{24} \mathrm{H}_{30} \mathrm{NaO}_{2} 373.2138$, found 373.2137. 
(3av)<smiles>CCOc1ccc(/C=C/C(C)(C)CCc2ccccc2)cc1</smiles>

The title compound was prepared according to the General Procedure D from the reaction of 2-methyl4-phenylbutan-2-ol 1a (32.8 mg, $0.20 \mathrm{mmol})$ with 4(2-bromovinyl)phenyl trifluoromethanesulfonate 2ae $(\mathrm{E} / \mathrm{Z}=7: 1,118.8 \mathrm{mg}, 0.36$ mmol).

$45.4 \mathrm{mg}, 57 \%$ yield, colorless oil.

${ }^{1} \mathbf{H}$ NMR (600 MHz, CDCl$) \delta 7.41(\mathrm{~d}, J=9.0 \mathrm{~Hz}, 2 \mathrm{H}), 7.28-7.25(\mathrm{~m}, 2 \mathrm{H}), 7.20(\mathrm{~d}$, $J=9.0 \mathrm{~Hz}, 2 \mathrm{H}), 7.17-7.16(\mathrm{~m}, 3 \mathrm{H}), 6.33(\mathrm{~d}, J=16.2 \mathrm{~Hz}, 1 \mathrm{H}), 6.24(\mathrm{~d}, J=16.2 \mathrm{~Hz}, 1$ H), 2.57-2.55 (m, $2 \mathrm{H}), 1.72-1.70(\mathrm{~m}, 2 \mathrm{H}), 1.17$ (s, $6 \mathrm{H})$.

${ }^{13}$ C NMR (150 MHz, CDCl 3$) \delta 148.2,142.9,142.5,138.4,128.4,128.3,127.6,125.7$, $124.5,121.4,118.8(\mathrm{q}, J=318.6 \mathrm{~Hz}), 45.1,36.7,31.3,27.1$.

${ }^{19}$ F NMR (376 MHz, CDCl3): $\delta-72.8$.

IR (neat, $\mathbf{c m}^{-1}$ ): 3066, 3032, 2965, 2868, 1649, 1605, 1500, 1456, 1426, 1251, 1213, $1180,1015,973,889,818,746,698,692$.

HRMS (ESI): $[\mathrm{M}+\mathrm{Na}]^{+}$calcd for $\mathrm{C}_{20} \mathrm{H}_{21} \mathrm{~F}_{3} \mathrm{NaO}_{3} \mathrm{~S} 421.1056$, found 421.1047 .

(E)-4,4-dimethyl-6-phenylhex-5-en-1-ol (3aw)

The title compound was prepared according to the General
Procedure D from the reaction of 4-methylpentane-1,4-diol $\mathbf{1 w}(23.6 \mathrm{mg}, 0.20 \mathrm{mmol})$ with (E)-(2-bromovinyl)benzene $\mathbf{2 a}(65.5 \mathrm{mg}, 0.36 \mathrm{mmol})$. $29.8 \mathrm{mg}, 73 \%$ yield, colorless oil.

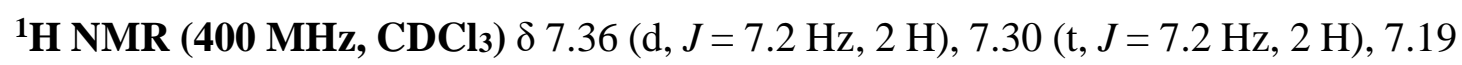
(t, $J=7.2 \mathrm{~Hz}, 1 \mathrm{H}), 6.29(\mathrm{~d}, J=16.4 \mathrm{~Hz}, 1 \mathrm{H}), 6.17(\mathrm{~d}, J=16.4 \mathrm{~Hz}, 1 \mathrm{H}), 3.62(\mathrm{t}, J=$ $6.4 \mathrm{~Hz}, 2 \mathrm{H}), 1.59-1.51$ (m, $2 \mathrm{H}), 1.45-1.41$ (m, $2 \mathrm{H}), 1.25$ (s, $1 \mathrm{H}), 1.11$ (s, $6 \mathrm{H})$.

${ }^{13}$ C NMR (100 MHz, CDCl3) $\delta 140.3,137.9,128.5,126.8,126.1,126.0,63.6,39.1$, $36.0,28.2,27.2$.

IR (neat, cm$^{-1}$ ): 3343, 3027, 2957, 2869, 1494, 1469, 1059, 1023, 747, 694.

HRMS (ESI): $[\mathrm{M}+\mathrm{Na}]^{+}$calcd for $\mathrm{C}_{14} \mathrm{H}_{20} \mathrm{NaO} 227.1406$, found 227.1396. 


\section{(E)-4,4-dimethyl-6-phenylhex-5-en-2-ol (3ax)}<smiles>CC(O)CC(C)(C)C=Cc1ccccc1</smiles>

The title compound was prepared according to the General Procedure D from the reaction of 2-methylpentane-2,4-diol $\mathbf{1 x}$ (23.6 mg, $0.20 \mathrm{mmol})$ with (E)-(2-bromovinyl)benzene 2a (65.5 mg, $0.36 \mathrm{mmol})$.

$18.4 \mathrm{mg}, 45 \%$ yield, colorless oil.

${ }^{1}$ H NMR (400 MHz, CDCl 3$) \delta 7.36(\mathrm{~d}, J=7.2 \mathrm{~Hz}, 2 \mathrm{H}), 7.29(\mathrm{t}, J=7.6 \mathrm{~Hz}, 2 \mathrm{H}), 7.20$ $(\mathrm{t}, J=7.6 \mathrm{~Hz}, 1 \mathrm{H}), 6.36(\mathrm{~d}, J=16.4 \mathrm{~Hz}, 1 \mathrm{H}), 6.30(\mathrm{~d}, J=16.4 \mathrm{~Hz}, 1 \mathrm{H}), 4.00-3.92(\mathrm{~m}$, $1 \mathrm{H}), 1.65\left(\mathrm{dd}, J_{1}=8.8 \mathrm{~Hz}, J_{2}=14.4 \mathrm{~Hz}, 1 \mathrm{H}\right), 1.60(\mathrm{~s}, 1 \mathrm{H}), 1.52\left(\mathrm{dd}, J_{1}=2.8 \mathrm{~Hz}, J_{2}\right.$ $=14.4 \mathrm{~Hz}, 1 \mathrm{H}), 1.19-1.16(\mathrm{~m}, 9 \mathrm{H})$.

${ }^{13}$ C NMR (100 MHz, CDCl $) \delta 140.5,137.5,128.5,127.1,126.3,126.1,65.7,52.5$, $36.0,29.1,26.5,24.9$.

IR (neat, $\mathbf{c m}^{-1}$ ): 3382, 2962, 2925, 1123, 1029, 937, 748, 694.

HRMS (ESI): [M+Na] ${ }^{+}$calcd for $\mathrm{C}_{14} \mathrm{H}_{20} \mathrm{NaO} 227.1406$, found 227.1398.

\section{(E)-4-(1-styrylcyclopentyl)butan-1-ol (3ay)}

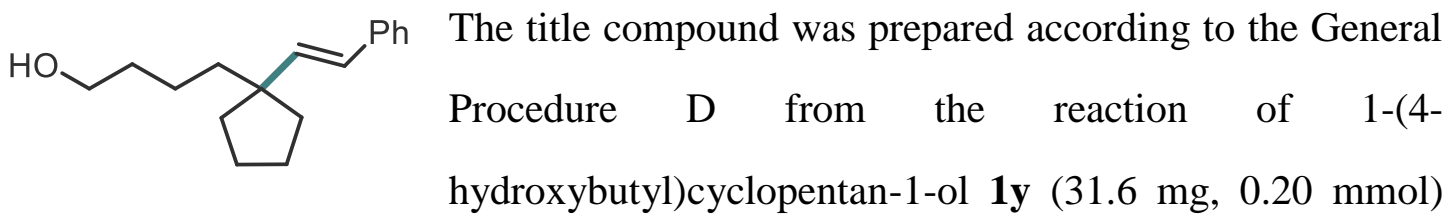

with (E)-(2-bromovinyl)benzene $\mathbf{2 a}(65.5 \mathrm{mg}, 0.36 \mathrm{mmol})$.

$25.4 \mathrm{mg}, 52 \%$ yield, colorless oil.

${ }^{1} \mathbf{H}$ NMR (600 MHz, CDCl 3$) \delta 7.36(\mathrm{~d}, J=7.2 \mathrm{~Hz}, 2 \mathrm{H}), 7.29(\mathrm{t}, J=7.2 \mathrm{~Hz}, 2 \mathrm{H}), 7.19$ $(\mathrm{t}, J=7.8 \mathrm{~Hz}, 1 \mathrm{H}), 6.27(\mathrm{~d}, J=16.2 \mathrm{~Hz}, 1 \mathrm{H}), 6.18(\mathrm{~d}, J=16.2 \mathrm{~Hz}, 1 \mathrm{H}), 3.62(\mathrm{t}, J=$ $6.6 \mathrm{~Hz}, 2 \mathrm{H}), 1.77-1.67$ (m, $2 \mathrm{H}), 1.66-1.63$ (m, 4 H), 1.57-1.46 (m, $6 \mathrm{H}), 1.35-1.30$ (m, $2 \mathrm{H}), 1.22(\mathrm{~s}, 1 \mathrm{H})$.

${ }^{13}$ C NMR (150 MHz, CDCl $) \delta 138.6,138.0,128.5,126.8,126.7,126.0,63.0,49.0$, $41.0,37.2,33.6,23.7,21.9$.

IR (neat, cm$^{-1}$ ): 3310, 3029, 2948, 2868, 1601, 1497, 1448, 1073, 1031, 969, 746, 693. HRMS (ESI): $[\mathrm{M}+\mathrm{Na}]^{+}$calcd for $\mathrm{C}_{17} \mathrm{H}_{24} \mathrm{NaO} 267.1719$, found 267.1718. 


\section{(E)-4-methyl-4-styrylcyclohexan-1-ol (3az)}

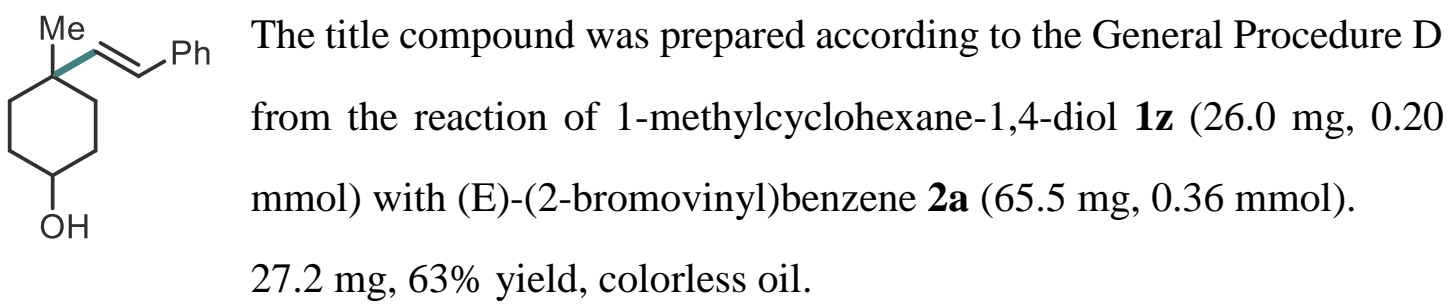

${ }^{1}$ H NMR (600 MHz, CDCl 3$) \delta$ 7.37-7.35 (m, 2 H), 7.31-7.28 (m, 2 H), 7.21-7.18 (m, $1 \mathrm{H}), 6.40-6.32$ (m, $1 \mathrm{H}), 6.20-6.17$ (m, $1 \mathrm{H}), 3.73-3.64$ (m, $1 \mathrm{H})$, 1.85-1.80 (m, $2 \mathrm{H})$, 1.63-1.35 (m, $7 \mathrm{H}), 1.12(\mathrm{~s}, 1.8 \mathrm{H}), 1.07(\mathrm{~s}, 1.2 \mathrm{H})$.

${ }^{13}$ C NMR (150 MHz, CDCl 3$) \delta 140.9,138.5,137.9,128.5,127.4,126.9,126.8,126.0$, $125.9,125.8,70.4,69.5,35.7,35.6,35.4,34.3,31.6,30.7$

IR (neat, $\mathbf{c m}^{-1}$ ): 3029, 2933, 2865, 1655, 1493, 1249, 1070, 1057, 967, 746, 693.

HRMS (ESI): [M+Na] ${ }^{+}$calcd for $\mathrm{C}_{15} \mathrm{H}_{20} \mathrm{NaO} 239.1406$, found 239.1409.

\section{(R,9E,19E)-18,18-dimethyl-20-phenylicosa-9,19-dien-7-ol (3ba)}

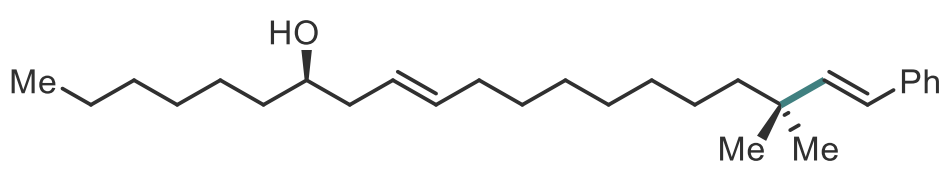

The title compound was prepared according to the General Procedure D from the reaction of (R,E)-2-methylnonadec-10-ene-2,13-diol 1aa $(62.5 \mathrm{mg}, 0.20 \mathrm{mmol})$ with (E)-(2-bromovinyl)benzene $\mathbf{2 a}(65.5 \mathrm{mg}, 0.36 \mathrm{mmol})$.

$46.2 \mathrm{mg}, 58 \%$ yield, colorless oil.

${ }^{1}$ H NMR (400 MHz, CDCl 3$) \delta 7.36(\mathrm{~d}, J=7.2 \mathrm{~Hz}, 2 \mathrm{H}), 7.30(\mathrm{t}, J=7.6 \mathrm{~Hz}, 2 \mathrm{H}), 7.18$ $(\mathrm{t}, J=7.2 \mathrm{~Hz}, 1 \mathrm{H}), 6.26(\mathrm{~d}, J=16.4 \mathrm{~Hz}, 1 \mathrm{H}), 6.18(\mathrm{~d}, J=16.4 \mathrm{~Hz}, 1 \mathrm{H}), 5.58-5.50(\mathrm{~m}$, $1 \mathrm{H})$, 5.42-5.29 (m, $1 \mathrm{H}), 3.61-3.57$ (m, $1 \mathrm{H}), 2.21-2.18(\mathrm{~m}, 2 \mathrm{H}), 2.06-1.99$ (m, $2 \mathrm{H})$, 1.47-1.25 (m, $23 \mathrm{H}), 1.08(\mathrm{~s}, 6 \mathrm{H}), 0.88$ (t, $J=6.8 \mathrm{~Hz}, 3 \mathrm{H})$.

${ }^{13}$ C NMR (100 MHz, CDCl3) $\delta$ 140.9, 138.1, 133.5, 128.4, 126.7, 126.0, 125.5, 125.1, $71.5,43.3,36.8,36.2,35.3,31.8,30.4,29.7,29.5,29.4,29.3,27.4,27.2,25.7,24.7$, 22.6, 14.1 .

IR (neat, $\mathbf{c m}^{-1}$ ): 3025, 2932, 2857, 1495, 1467, 1383, 1364, 1074, 1049, 969, 910 , 747, 725, 693.

HRMS (ESI): $[\mathrm{M}+\mathrm{Na}]^{+}$calcd for $\mathrm{C}_{28} \mathrm{H}_{46} \mathrm{NaO} 421.3441$, found 421.3440 . 


\section{(E)-2-(4-(3,3-dimethyl-5-phenylpent-4-en-1-yl)phenoxy)propane-1,3-diol (3bb)}<smiles>CC(C)(/C=C/c1ccccc1)CCc1ccc(OC(CO)CO)cc1</smiles>

The title compound was prepared according to the General Procedure D from the reaction of 2(4-(3-hydroxy-3-methylbutyl)phenoxy)propane1,3-diol 1ab (50.8 mg, $0.20 \mathrm{mmol}$ ) with (E)-(2-bromovinyl)benzene 2a (65.5 mg, 0.36 mmol).

$42.9 \mathrm{mg}, 63 \%$ yield, colorless oil.

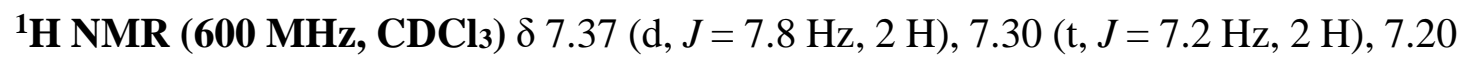
(t, $J=7.2 \mathrm{~Hz}, 1 \mathrm{H}), 7.08(\mathrm{~d}, J=8.4 \mathrm{~Hz}, 2 \mathrm{H}), 6.88(\mathrm{~d}, J=8.4 \mathrm{~Hz}, 2 \mathrm{H}), 6.33$ (d, $J=$ 16.2 Hz, $1 \mathrm{H}), 6.22$ (d, $J=16.2 \mathrm{~Hz}, 1 \mathrm{H}), 4.37-4.34$ (m, $1 \mathrm{H}), 3.90-3.84$ (m, 4 H), 2.532.50 (m, 2 H), 2.15-2.12 (m, 2 H), 1.67-1.65 (m, 2 H), 1.16 (s, $6 \mathrm{H})$.

${ }^{13}$ C NMR (150 MHz, CDCl 3$) \delta 155.6,140.1,137.9,136.6,129.4,128.5,126.8,126.2$, 126.0, 116.3, 78.6, 62.1, 45.4, 36.4, 30.4, 27.2.

IR (neat, $\mathbf{c m}^{-1}$ ): 3027, 2958, 2868, 1612, 1511, 1449, 1364, 1239, 1177, 1046, 971 , 937, 831, 747, 695 .

HRMS (ESI): [M+Na $]^{+}$calcd for $\mathrm{C}_{22} \mathrm{H}_{28} \mathrm{NaO}_{3} 363.1931$, found 363.1929.

(E)-2-(4-(3,3-dimethyl-5-phenylpent-4-en-1-yl)phenoxy)butane-1,3-diol (3bc)<smiles>CC(O)C(CO)Oc1ccc(CCC(C)(C)/C=C/c2ccccc2)cc1</smiles>

The title compound was prepared according to the General Procedure D from the reaction of 2(4-(3-hydroxy-3-methylbutyl)phenoxy)butane-

1,3-diol 1ac (53.6 mg, $0.20 \mathrm{mmol}$ ) with (E)-(2-bromovinyl)benzene 2a (65.5 mg, 0.36 mmol).

$43.2 \mathrm{mg}, 61 \%$ yield, colorless oil.

${ }^{1} \mathbf{H}$ NMR (600 MHz, CDCl 3$) \delta 7.37(\mathrm{~d}, J=7.2 \mathrm{~Hz}, 2 \mathrm{H}), 7.31(\mathrm{t}, J=7.8 \mathrm{~Hz}, 2 \mathrm{H}), 7.20$ (t, $J=7.2 \mathrm{~Hz}, 1 \mathrm{H}), 7.08(\mathrm{~d}, J=8.4 \mathrm{~Hz}, 2 \mathrm{H}), 6.90(\mathrm{~d}, J=8.4 \mathrm{~Hz}, 2 \mathrm{H}), 6.33(\mathrm{~d}, J=$ 16.2 Hz, $1 \mathrm{H}), 6.22$ (d, $J=16.2 \mathrm{~Hz}, 1 \mathrm{H}), 4.14-4.10$ (m, 2 H), 3.94-3.91 (m, $1 \mathrm{H}), 3.81$ 3.78 (m, 1 H), 2.53-2.50 (m, 2 H), 2.35 (s, 1 H), 1.97 (s, 1 H), 1.68-1.60 (m, 2 H), 1.29 $(\mathrm{d}, J=6.0 \mathrm{~Hz}, 3 \mathrm{H}), 1.16(\mathrm{~s}, 6 \mathrm{H})$.

${ }^{13}$ C NMR (150 MHz, CDCl 3$) \delta 156.2,140.1,137.9,136.7,129.4,128.5,126.9,126.2$, 
$126.0,116.4,82.6,67.8,61.7,45.4,36.4,30.4,27.2,19.0$.

IR (neat, $\mathbf{c m}^{-1}$ ): 2932, 2858, 1610, 1510, 1448, 1405, 1237, 1177, 1139, 1406, 971, $910,731,695$.

HRMS (ESI): [M+Na] ${ }^{+}$calcd for $\mathrm{C}_{23} \mathrm{H}_{30} \mathrm{NaO}_{3}$ 377.2087, found 377.2086.

(7S,8R,E)-16,16-dimethyl-18-phenyloctadec-17-ene-1,7,8-triol (3bd)<smiles>C[C@@](/C=C/c1ccccc1)(CCCO)CCCCCCCC(O)[C@H](O)CCCCCCO</smiles>

The title compound was prepared according to the General Procedure D from the reaction of (7S,8R)-16-methylheptadecane-1,7,8,16-tetraol 1ad (63.7 mg, $0.20 \mathrm{mmol})$ with (E)-(2-bromovinyl)benzene $2 \mathbf{a}(65.5 \mathrm{mg}, 0.36 \mathrm{mmol})$.

$56.6 \mathrm{mg}, 70 \%$ yield, colorless oil.

${ }^{1}$ H NMR (600 MHz, MeOD) $\delta 7.33(\mathrm{~d}, J=7.2 \mathrm{~Hz}, 2 \mathrm{H}), 7.26(\mathrm{t}, J=7.2 \mathrm{~Hz}, 2 \mathrm{H}), 7.15$ $(\mathrm{t}, J=7.2 \mathrm{~Hz}, 1 \mathrm{H}), 6.28(\mathrm{~d}, J=16.2 \mathrm{~Hz}, 1 \mathrm{H}), 6.19(\mathrm{~d}, J=16.2 \mathrm{~Hz}, 1 \mathrm{H}), 3.54(\mathrm{t}, J=$ $6.6 \mathrm{~Hz}, 2 \mathrm{H}), 3.36-3.35$ (m, $2 \mathrm{H}), 1.55-1.48$ (m, $6 \mathrm{H}), 1.40-1.28$ (m, $21 \mathrm{H}), 1.09$ (s, 6 $\mathrm{H})$.

${ }^{13}$ C NMR (100 MHz, MeOD) $\delta$ 140.1, 138.0, 128.1, 126.3, 125.7, 125.5, 73.9, 73.8, 61.6, 43.0, 35.6, 32.5, 32.4, 32.2, 30.1, 29.4, 29.3, 29.2, 26.3, 25.7, 25.6, 25.5, 24.4.

IR (neat, cm$^{-1}$ ): 2961, 2857, 2540, 1640, 1450, 1366, 1200, 1178, 969, 693.

HRMS (ESI): [M+Na] ${ }^{+}$calcd for $\mathrm{C}_{26} \mathrm{H}_{44} \mathrm{NaO}_{3} 427.3183$, found 427.3184 .

(E)-(4-(3,3-dimethyl-5-phenylpent-4-en-1-yl)phenyl)methanol (3be)

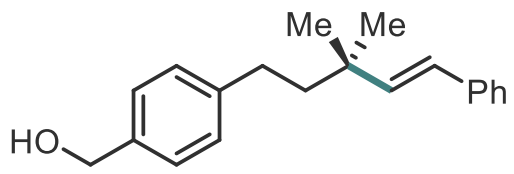

The title compound was prepared according to the

General Procedure D from the reaction of 4-(4(hydroxymethyl)phenyl)-2-methylbutan-2-ol 1ae (38.8 mg, $0.20 \mathrm{mmol}$ ) with (E)-(2-bromovinyl)benzene 2a (65.5 mg, $0.36 \mathrm{mmol}$ ).

$28.0 \mathrm{mg}, 50 \%$ yield, colorless oil.

${ }^{1} \mathbf{H}$ NMR (600 MHz, CDCl 3$) \delta 7.37(\mathrm{~d}, J=7.2 \mathrm{~Hz}, 2 \mathrm{H}), 7.31(\mathrm{t}, J=7.8 \mathrm{~Hz}, 2 \mathrm{H}), 7.26$ $(\mathrm{d}, J=8.4 \mathrm{~Hz}, 2 \mathrm{H}), 7.20(\mathrm{t}, J=7.8 \mathrm{~Hz}, 1 \mathrm{H}), 7.17(\mathrm{~d}, J=7.8 \mathrm{~Hz}, 2 \mathrm{H}), 6.33(\mathrm{~d}, J=$ $16.2 \mathrm{~Hz}, 1 \mathrm{H}), 6.22(\mathrm{~d}, J=16.2 \mathrm{~Hz}, 1 \mathrm{H}), 4.64(\mathrm{~s}, 2 \mathrm{H}), 2.59-2.56(\mathrm{~m}, 2 \mathrm{H}), 1.70-1.68(\mathrm{~m}$, $2 \mathrm{H}), 1.58(\mathrm{~s}, 1 \mathrm{H}), 1.17$ (s, $6 \mathrm{H})$. 
${ }^{13}$ C NMR (150 MHz, CDCl3) $\delta$ 142.7, 140.1, 138.1, 137.9, 128.5, 128.4, 127.2, 126.9, 126.2, 126.0, 65.3, 45.3, 36.5, 31.1, 27.2 .

IR (neat, cm-1): 3027, 2960, 2865, 1515, 1494, 1385, 1364, 1204, 1031, 1016, 971, $816,746,693$,

HRMS (ESI): $[\mathrm{M}+\mathrm{Na}]^{+}$calcd for $\mathrm{C}_{20} \mathrm{H}_{24} \mathrm{NaO} 303.1719$, found303.1717.

(3R,5R,6S,8R,9S,10R,12S,13R,14S,17R)-17-((R,E)-5,5-dimethyl-7-phenylhept-6en-2-yl)-10,13-dimethylhexadecahydro-1H-cyclopenta[a]phenanthrene-3,6,12triol (3bf)

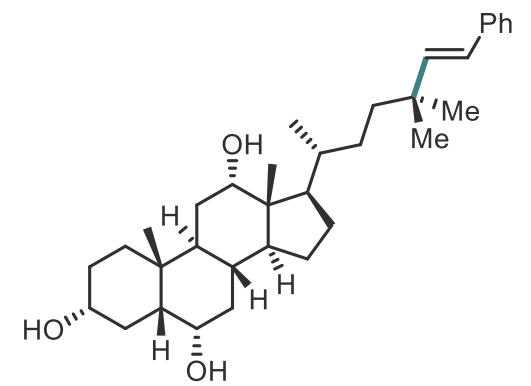

The title compound was prepared according to the General Procedure D from the reaction of (3R,5R,6S,8R,9S, 10R,12S,13R,14S,17R)-17-((R)-5hydroxy-5-methylhexan-2-yl)-10,13dimethylhexadecahydro-1Hcyclopenta[a]phenanthrene-3,6,12-triol 1af $(84.5 \mathrm{mg}, 0.20 \mathrm{mmol})$ with (E)-(2bromovinyl)benzene $2 \mathbf{a}(65.5 \mathrm{mg}, 0.36 \mathrm{mmol})$.

$63.0 \mathrm{mg}, 62 \%$ yield.

${ }^{1}$ H NMR (600 MHz, MeOD) $\delta 7.33(\mathrm{~d}, J=7.2 \mathrm{~Hz}, 2 \mathrm{H}), 7.26(\mathrm{t}, J=7.8 \mathrm{~Hz}, 2 \mathrm{H}), 7.15$ (t, $J=7.2 \mathrm{~Hz}, 1 \mathrm{H}), 6.27$ (d, $J=16.2 \mathrm{~Hz}, 1 \mathrm{H}), 6.17$ (d, $J=16.2 \mathrm{~Hz}, 1 \mathrm{H}), 3.93(\mathrm{~s}, 1 \mathrm{H})$, 3.78-3.77 (m, 1 H), 3.39-3.34 (m, 1 H), 2.30-2.21 (m, 2 H), 1.98-1.78 (m, 6 H), 1.71$1.50(\mathrm{~m}, 9 \mathrm{H}), 1.43-1.21(\mathrm{~m}, 7 \mathrm{H}), 1.08(\mathrm{~s}, 6 \mathrm{H}), 1.00(\mathrm{~d}, J=6.6 \mathrm{~Hz}, 3 \mathrm{H}), 0.90(\mathrm{~s}, 3 \mathrm{H})$, $0.68(\mathrm{~s}, 3 \mathrm{H})$.

${ }^{13}$ C NMR (150 MHz, MeOD) $\delta$ 140.3, 138.1, 128.1, 126.4, 125.8, 125.6, 72.7, 71.5, 67.7, 46.6, 46.0, 41.8, 41.6, 39.6, 39.1, 39.0, 36.2, 35.7, 35.1, 34.5, 34.4, 30.4, 29.8, $28.2,27.4,26.5,26.3,22.8,21.8,16.8,11.6$.

IR (neat, $\mathbf{c m}^{-1}$ ): 2939, 2865, 1740, 1610, 1478, 1329, 1298, 1280, 1230, 1121, 1069, 927, 744, 687.

HRMS (ESI): $[\mathrm{M}+\mathrm{Na}]^{+}$calcd for $\mathrm{C}_{34} \mathrm{H}_{52} \mathrm{NaO}_{3} 531.3809$, found 531.3803. 


\section{Mechanistic Investigation}

\subsection{Experiments that support a radical addition process}

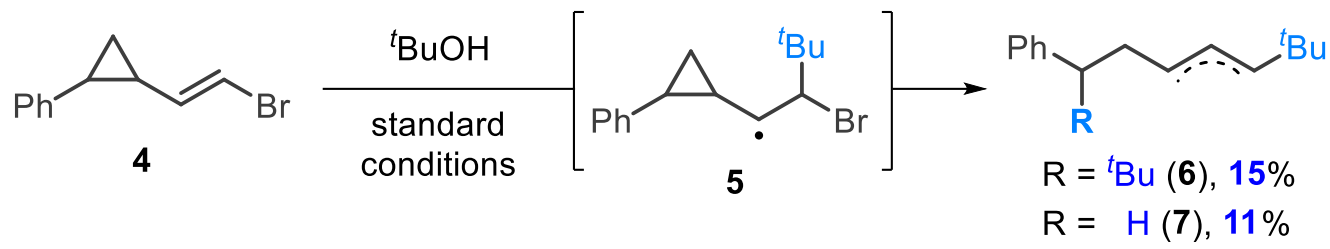

The reaction was performed according to the general procedure $\mathrm{D}$ for the Ti-catalyzed coupling reactions. 2-Methylpropan-2-ol 1 b $(29.2 \mathrm{mg}, 0.40 \mathrm{mmol})$ and (2-(2bromovinyl)cyclopropyl)benzene 4 (44.4 mg, $0.20 \mathrm{mmol}$ ) were used. Compounds 6 (mixture of alkene isomers) and 7 (mixture of alkene isomers) were obtained in 15\% $(7.8 \mathrm{mg}$ ) and $11 \%$ (4.5 mg) yields, respectively. The characterization data of the major isomer of $\mathbf{6}$ and 7 were provided, which were purified by preparative HPLC.

(E)-(2,2,8,8-tetramethylnon-5-en-3-yl)benzene (the major isomer of 6)<smiles>CC(C)(C)CC=CCC(Br)c1ccccc1</smiles>

${ }^{1}$ H NMR (400 MHz, CDCl 3$) \delta 7.23(\mathrm{t}, J=7.2 \mathrm{~Hz}, 2 \mathrm{H}), 7.16-7.10(\mathrm{~m}, 3 \mathrm{H})$, 5.32-5.24 (m, $1 \mathrm{H})$, 5.09-5.02 (m, $1 \mathrm{H}), 2.53-2.34(\mathrm{~m}, 3 \mathrm{H}), 1.71-1.60$ (m, $2 \mathrm{H}), 0.88$ (s, $9 \mathrm{H}), 0.64$ (s, $9 \mathrm{H})$.

${ }^{13}$ C NMR (100 MHz, CDCl $) \delta 142.6,131.7,129.8,128.3,127.4,125.7,57.2,47.0$, $33.8,32.9,30.6,29.0,28.3$.

IR (neat, $\mathbf{c m}^{-1}$ ): 2960, 2361, 1636, 1364, 969, 747, 696, 642.

HRMS (ESI): [M] ${ }^{+}$calcd for $\mathrm{C}_{19} \mathrm{H}_{30} 258.2348$, found 258.2351 .

(E)-(6,6-dimethylhept-3-en-1-yl)benzene (the major isomer of 7, known compound)

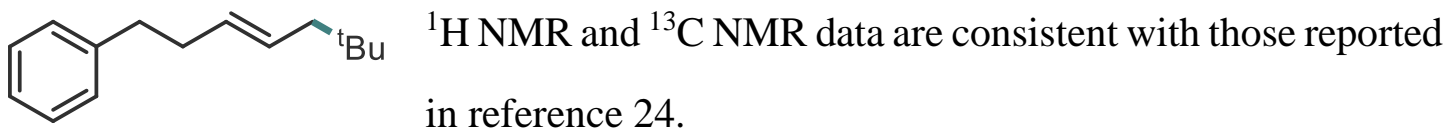
NMR (600 MHz, CDCl $) \delta 7.27$ (t, $J=7.8$ Hz, 2 H), 7.19-7.16 (m, 3 H), 5.48-5.39 (m, $2 \mathrm{H}), 2.68(\mathrm{t}, J=7.8 \mathrm{~Hz}, 2 \mathrm{H}), 2.35-2.31(\mathrm{~m}, 2 \mathrm{H}), 1.85(\mathrm{~d}, J=6.6 \mathrm{~Hz}, 2 \mathrm{H}), 0.83$ $(\mathrm{s}, 9 \mathrm{H})$. 
${ }^{13}$ C NMR (150 MHz, CDCl $) \delta 142.1,131.6,128.5,128.2,128.1,125.7,47.1,36.2$, $34.5,30.8,29.2$.

\subsection{Experiments to probe debromination process}

\subsubsection{Reaction of alcohol 1a with vinyl bromide 8}

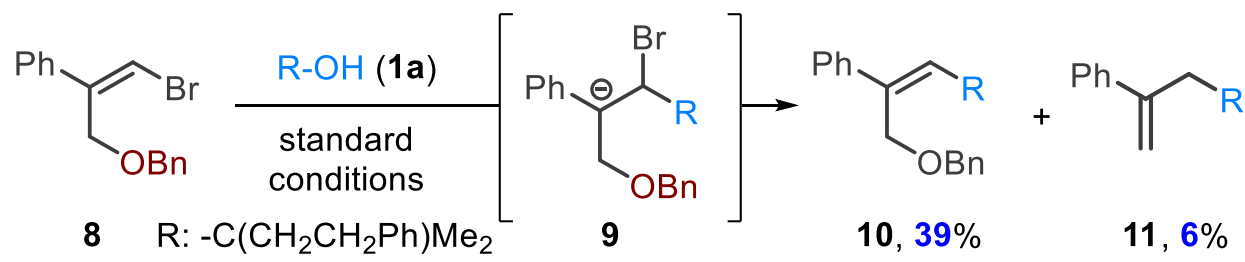

The reaction was performed according to the general procedure $\mathrm{D}$ for the Ti-catalyzed coupling reactions. 2-Methyl-4-phenylbutan-2-ol 1a (32.8 mg, $0.20 \mathrm{mmol})$ and (Z)-(3(benzyloxy)-1-bromoprop-1-en-2-yl)benzene 8 (108.7 mg, $0.36 \mathrm{mmol})$ were used. The reaction afforded compound $\mathbf{1 0}$ in $39 \%$ yield $(28.9 \mathrm{mg})$ and compound $\mathbf{1 1}$ in $6 \%$ yield (3.2 mg). They are colorless oil.

(Z)-(6-(benzyloxy)-3,3-dimethylhex-4-ene-1,5-diyl)dibenzene (10)

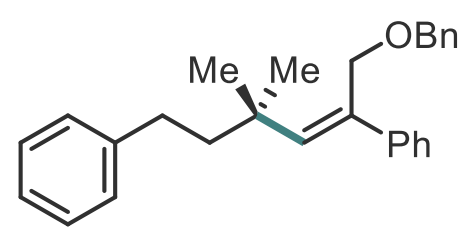

${ }^{1}$ H NMR (400 MHz, CDCl3) $\delta$ 7.41-7.39 (m, 2 H), 7.31-7.28 (m, 3 H), 7.26-7.21 (m, $7 \mathrm{H})$, 7.17-7.13 (m, $3 \mathrm{H}), 5.89$ (s, $1 \mathrm{H}), 4.50$ (s, $2 \mathrm{H}), 4.49$ (s, $2 \mathrm{H})$, 2.66-2.61 (m, $2 \mathrm{H})$, 1.78-1.74 (m, $2 \mathrm{H}), 1.26$ (s, $6 \mathrm{H})$.

${ }^{13}$ C NMR (100 MHz, CDCl3) $\delta$ 143.5, 143.0, 142.9, 138.1, 136.8, 128.3 (2C), 128.2, $128.1,127.9,127.5,126.7,126.6,125.6,72.5,67.0,46.9,36.7,31.6,29.3$.

IR (neat, $\mathbf{c m}^{-1}$ ): 3030, 2928, 2955, 2864, 1949, 1867, 1601, 1455, 1362, 1265, 1217 , 1075, 1029, 736, 697.

HRMS (ESI): $[\mathrm{M}+\mathrm{Na}]^{+}$calcd for $\mathrm{C}_{27} \mathrm{H}_{30} \mathrm{NaO} 393.2189$, found 393.2189. 


\section{(3,3-Dimethylhex-5-ene-1,5-diyl)dibenzene (11)}

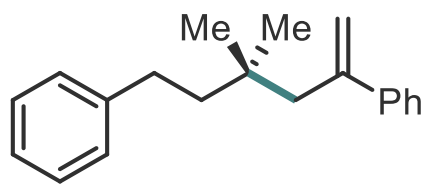

${ }^{1}$ H NMR (400 MHz, CDCl $) \delta 7.39(\mathrm{~d}, J=6.8 \mathrm{~Hz}, 2 \mathrm{H}), 7.31(\mathrm{t}, J=6.8 \mathrm{~Hz}, 2 \mathrm{H}), 7.23$ $(\mathrm{t}, J=7.6 \mathrm{~Hz}, 3 \mathrm{H}), 7.14(\mathrm{t}, J=7.6 \mathrm{~Hz}, 1 \mathrm{H}), 7.01(\mathrm{~d}, J=6.8 \mathrm{~Hz}, 2 \mathrm{H}), 5.25(\mathrm{~d}, J=2.0$ $\mathrm{Hz}, 1 \mathrm{H}), 5.06(\mathrm{~d}, J=2.0 \mathrm{~Hz}, 1 \mathrm{H}), 2.54(\mathrm{~s}, 2 \mathrm{H}), 2.53-2.48(\mathrm{~m}, 2 \mathrm{H}), 1.46-1.42(\mathrm{~m}, 2$ $\mathrm{H}), 0.83(\mathrm{~s}, 6 \mathrm{H})$.

${ }^{13}$ C NMR (100 MHz, CDCl3) $\delta$ 147.3, 143.9, 143.3, 128.3, 128.2, 128.1, 127.0, 126.6, $125.5,116.9,46.7,44.6,34.4,30.8,27.7$.

IR (neat, $\mathbf{c m}^{-1}$ ): 3060, 3030, 2963, 2873, 1625, 1496, 1470, 1386, 1366, 1075, 1030, $902,779,698$.

HRMS (EI): $[\mathrm{M}]^{+}$calcd for $\mathrm{C}_{20} \mathrm{H}_{24} 264.1878$, found 264.1879 .

\subsubsection{Reaction of alcohol 1a with alkene 12}<smiles>C=C(COc1ccccc1)c1ccccc1</smiles>

12

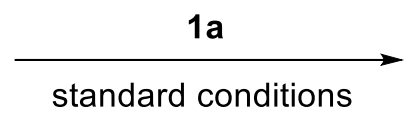

standard conditions

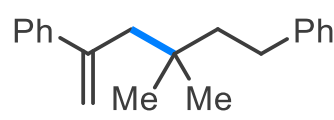

$11,43 \%$

The reaction was performed according to the general procedure $\mathrm{D}$ for the Ti-catalyzed coupling reactions. 2-Methyl-4-phenylbutan-2-ol 1a $(32.8 \mathrm{mg}, 0.20 \mathrm{mmol})$ and (3(benzyloxy)prop-1-en-2-yl)benzene 12 (80.7 $\mathrm{mg}, 0.36 \mathrm{mmol})$ were used. the reaction afforded compound 11 in $43 \%$ yield $(22.7 \mathrm{mg})$ as a colorless oil. 


\section{References}

(1) Armarego, W. L. F.; Chai, C. C. L. Purification of laboratory chemicals, 5th ed., Butterworth- Heinemann, 2003, 61-68.

(2) Xie, H.; Guo, J.; Wang, Y.-Q.; Wang, K.; Guo, P.; Su, P.-F.; Wang, X.; Shu, X.-Z. Radical dehydroxylative alkylation of tertiary alcohols by Ti Catalysis. J. Am. Chem. Soc. 2020, 142, 16787-16794.

(3) Saranya, S.; Chand, S. S.; Gopalan, G.; Jijitha, V.; Radhakrishnan, K. V. A Facile Access to trans-3-Styryl-4-hydrazinocyclopentenes via Palladium-Catalyzed Ring Opening of Diazanorbornenes with (Z)- $\beta$-Bromostyrenes/2,3 Dibromohydrocinnamic Acids. Synthesis. 2018, 50, 184-192.

(4) Li, C.-T.; Yuan, X.; Tang, Z.-Y. Transition metal free decarboxylative fluorination of cinnamic acids with selectfluor ${ }^{\circledR}$. Tetrahedron Letters. 2016, 57, 5624-5627.

(5) You, H.-W.; Lee, K.-J. Halodecarboxylation of $\alpha, \beta$-Unsaturated Carboxylic Acids bearing Aryl and Styrenyl Group at $\beta$-Carbon with Oxone ${ }^{\circledR}$ and Sodium Halide. Synlett. 2001, 1, 105-107.

(6) Bull, J. A.; Mousseau, J. J.; Charette, A. B. Convenient One-Pot Synthesis of (E)$\beta$-Aryl Vinyl Halides from Benzyl Bromides and Dihalomethanes. Org. Lett. 2008, $10,5485-5488$.

(7) Yu, W.; Chen, L.; Tao, J.; Wang, T.; Fu, J. Dual nickel- and photoredox-catalyzed reductive cross-coupling of aryl vinyl halides and unactivated tertiary alkyl bromides. Chem. Commun. 2019, 55, 5918-5921.

(8) Cheung, C. W.; Buchwald, S. L. Room Temperature Copper(II)-Catalyzed Oxidative Cyclization of Enamides to 2,5-Disubstituted Oxazoles via Vinylic $\mathrm{C}-\mathrm{H}$ Functionalization. J. Org. Chem. 2012, 77, 7526-7537.

(9) Lukas, N.; Sigrid, L.; Pascal, O.; Richard, G.; Cornella, J. A. Highly Reduced NiLi-Olefin Complex for Catalytic Kumada-Corriu Cross-Couplings. J. Am. Chem. Soc. 2018, 140, 13628-13633.

(10)Liu, J.; Gong, H.; Zhu, S. Nickel-catalyzed, regio- and enantioselective benzylic 
alkenylation of olefins with alkenyl bromide. Angew.Chem. Int.Ed. 2021, 60, 40604064.

(11)Fiandanese, V.; Bottalico, D.; Marchese, G.; Punzi, A. Synthesis of naturally occurring polyacetylenes via a bis-silylated diyne. Tetrahedron, 2006, 62, 51265132.

(12)Molloy, J. J.; Metternich, J. B.; Daniliuc, C. G.; Watson, A. J. B.; Gilmour, R. Contra-Thermodynamic, Photocatalytic $\mathrm{E} \rightarrow \mathrm{Z}$ Isomerization of Styrenyl Boron Species: Vectors to Facilitate Exploration of Two- Dimensional Chemical Space. Angew. Chem. Int. Ed. 2018, 57, 3168-3172.

(13)Qian, M.; Huang, Z.; Negishi, E. Use of $\mathrm{InCl}_{3}$ as a Cocatalyst and a $\mathrm{Cl}_{2} \mathrm{Pd}$ (DPEphos)-P(2-Furyl) ${ }_{3}$ Catalyst System for One-Pot Hydrometalation-CrossCoupling and Carbometalation-Cross-Coupling Tandem Processes. Org. Lett. 2004, $6,1531-1534$.

(14)Pintu, M.; Debasish, K.; Rajdip, R.; Brindaban, R. A Direct Synthesis of Selenophenes by Cu-Catalyzed One-Pot Addition of a Selenium Moiety to (E,E)1,3-Dienyl Bromides and Subsequent Nucleophilic Cyclization. Org. Lett. 2014, 16, $4122-4125$.

(15)Daniel, M.; Alexandre, A. Formation of Quaternary Stereogenic Centers by Copper-Catalyzed Asymmetric Conjugate Addition Reactions of Alkenylaluminums to Trisubstituted Enones. Chem. Eur. J. 2013, 19, 15226-15239.

(16) Yeshwant, R. B.; Chang, S.-H.; Chang, C.-T.; Yan, T.-H. A new entry of highly nucleophilic $\mathrm{CHBr}_{3}-\mathrm{TiCl}_{4}-\mathrm{Mg}$ system for the stereoselective synthesis of 1-alkenyl bromides. Tetrahedron. 2012, 68, 4846-4851.

(17) Ye, Y.; Chen, H.; Yao, K.; Gong, H. Iron-Catalyzed Reductive Vinylation of Tertiary Alkyl Oxalates with Activated Vinyl Halides. Org. Lett. 2020, 22, 20702075.

(18)Huang, J.; Hu, G.; An, S.; Chen, D.; Li, M.; Li, P. Synthesis of N-Alkylpyridin-4ones and Thiazolo[3,2-a]pyridin-5-ones through Pummerer-Type Reactions. J. Org. Chem. 2019, 84, 9758-9769. 
(19)Luo, X.; Chen, X.; Chen, L.; Zhang, K.; Li, Y. Xanthate-Mediated Synthesis of (E)Alkenes by Semi-Hydrogenation of Alkynes Using Water as the Hydrogen Donor. Chem. Commun. 2019, 55, 2170-2713.

(20)Cabrero-Antonino, R.; Tejeda-Serrano, M.; Quesada, M.; Vidal-Moya, J. A.; Leyva-Perez, A.; Corma, A. Bimetallic nanosized solids with acid and redox properties for catalytic activation of $\mathrm{C}-\mathrm{C}$ and $\mathrm{C}-\mathrm{H}$ bonds. Chem. Sci. 2017, 8, 689-696.

(21)Xu, K.; Tan, Z.; Zhang, H.; Liu, J.; Zhang, S.; Wang, Z. Photoredox catalysis enabled alkylation of alkenyl carboxylic acids with $\mathrm{N}$-(acyloxy)phthalimide via dual decarboxylation. Chem. Commun. 2017, 53, 10719-10722.

(22)Liu, J.-T.; Jang, Y.-J.; Shih, Y.-K.; Hu, S.-R.; Chu, C.-M.; Yao, C.-F. Novel Synthesis of Alkenes via Triethylborane-Induced Free-Radical Reactions of Alkyl Iodides and â-Nitrostyrenes. J. Org. Chem. 2001, 66, 6021-6028.

(23)Jang, Y.-G.; Yan, M.-C.; Lin, Y.-F.; Yao, C.-F. A Simple Radical Addition Elimination Route to Geometrically Pure (E)-Alkene and Chromanone Derivatives via â-Nitrostyrene. J. Org. Chem. 2004, 69, 3961-3963.

(24)Chen, X.; Cheng, Z.; Guo, J.; Lu, Z. Asymmetric remote C-H borylation of internal alkenes via alkene isomerization. Nat. Commun.2018, 9, 3939-3046. 


\section{Copies of NMR Spectra}

1f; ${ }^{1} \mathrm{H}$ NMR (600 MHz, $\left.\mathrm{CDCl}_{3}\right) ;{ }^{13} \mathrm{C}$ NMR (150 MHz, $\left.\mathrm{CDCl}_{3}\right)$
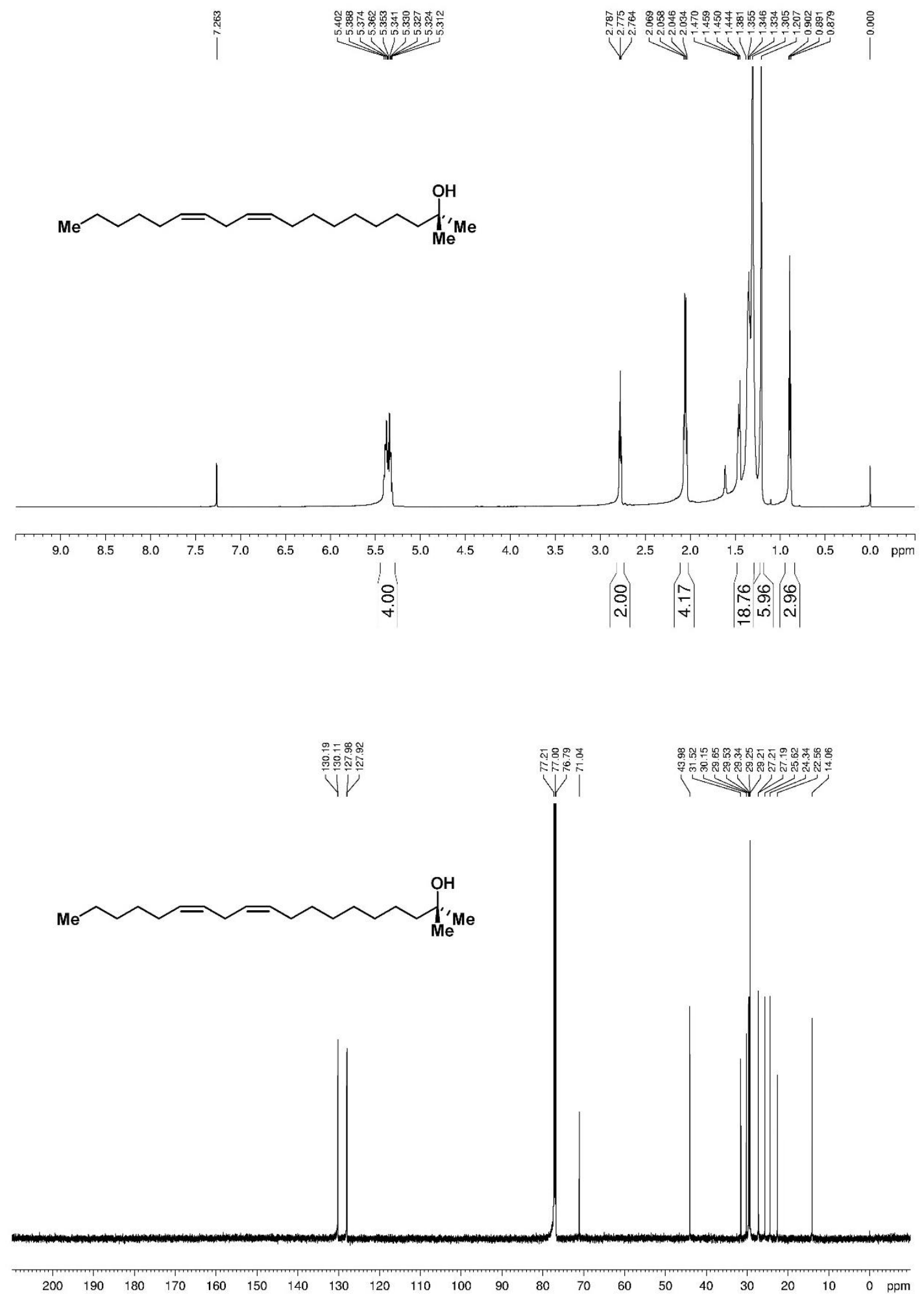
1aa; ${ }^{1} \mathrm{H}$ NMR (600 MHz, CDCl 3$) ;{ }^{13} \mathrm{C}$ NMR (150 MHz, CDCl $)$
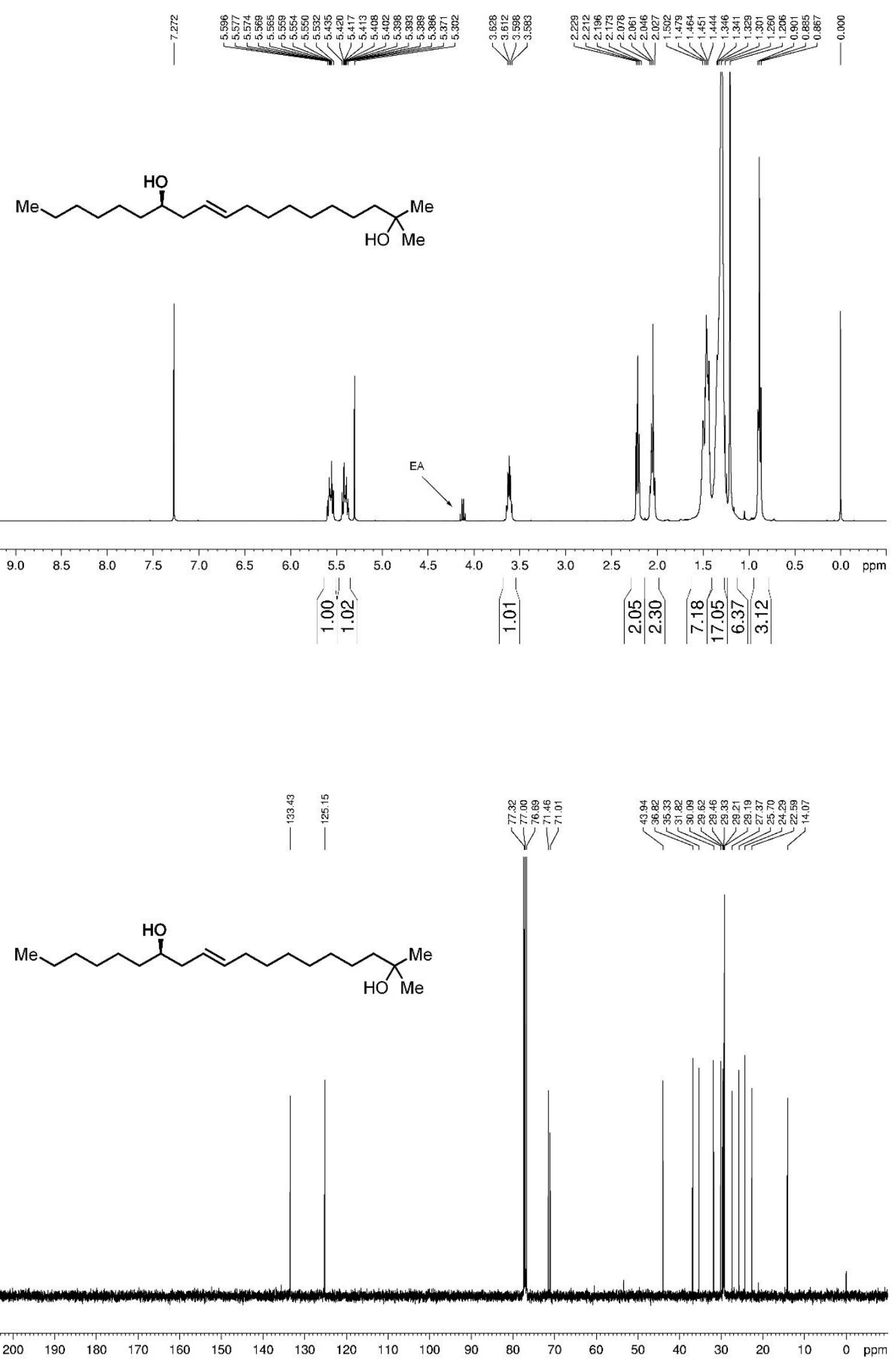
1ab; ${ }^{1} \mathrm{H}$ NMR (400 MHz, MeOD); ${ }^{13} \mathrm{C}$ NMR (100 MHz, MeOD)

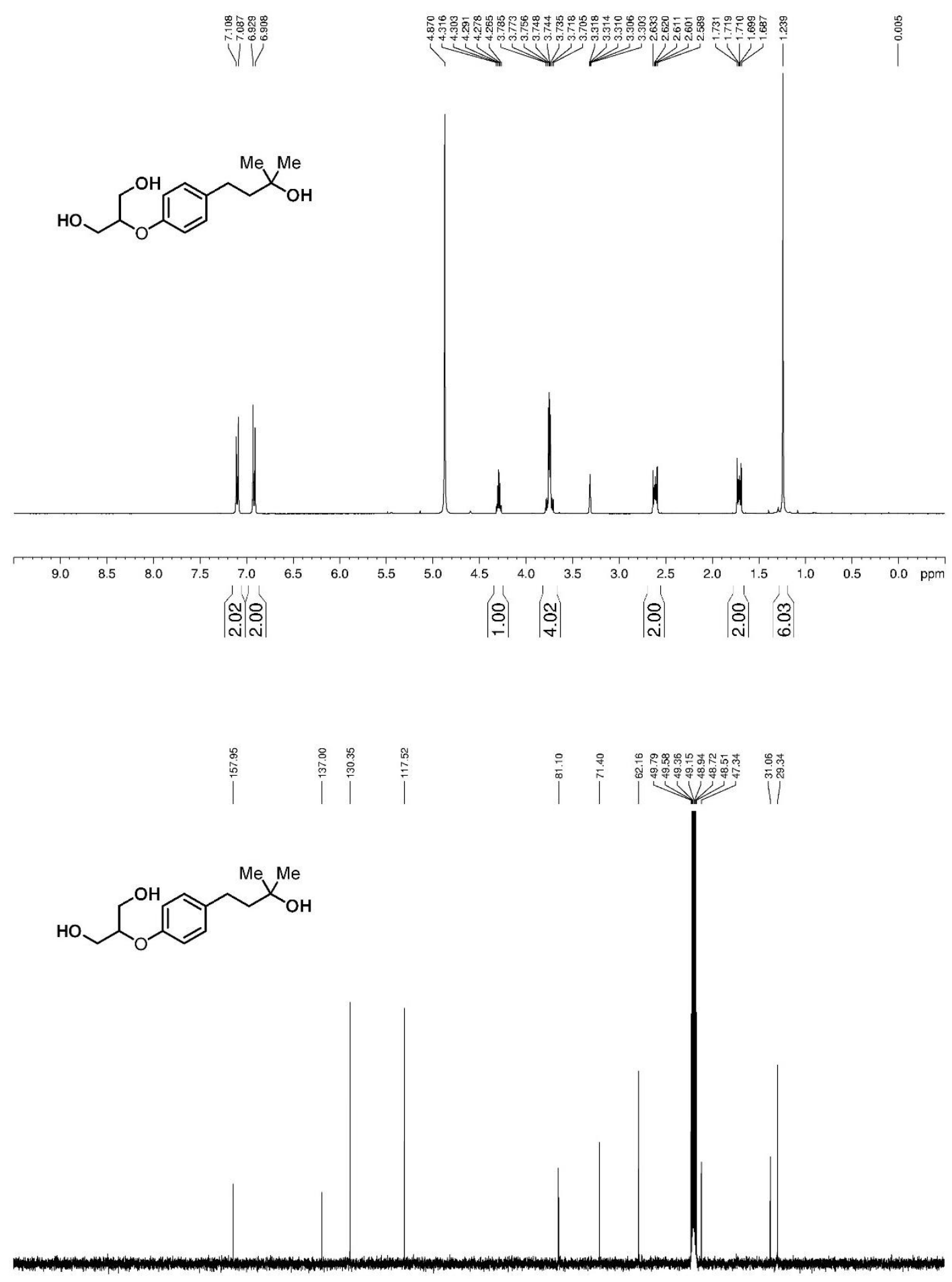

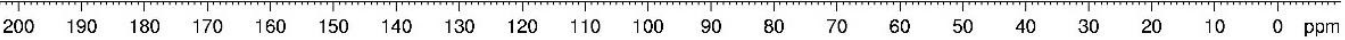


1ac; ${ }^{1} \mathrm{H}$ NMR (400 MHz, MeOD); ${ }^{13} \mathrm{C}$ NMR (100 MHz, MeOD)
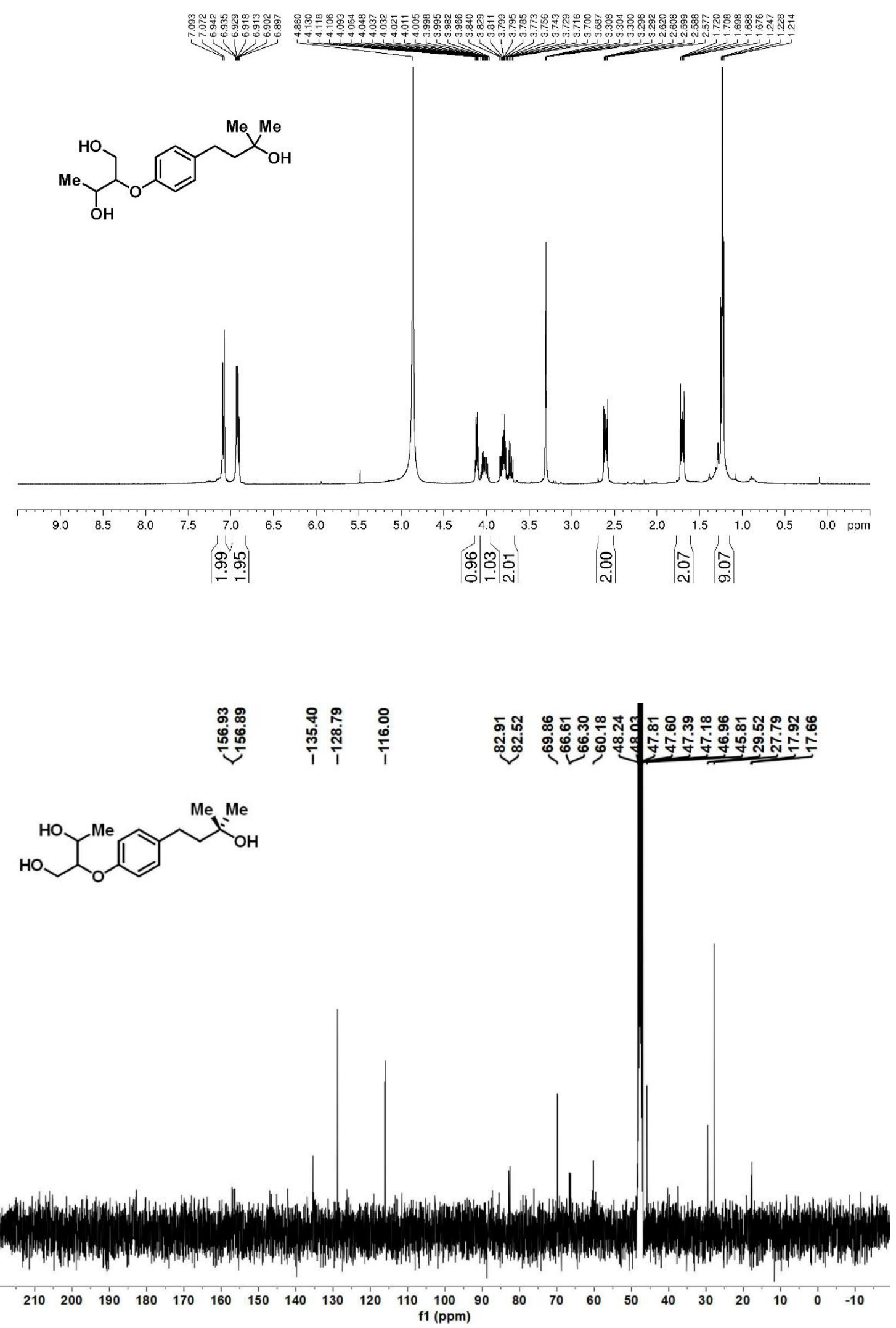
1ad; ${ }^{1} \mathrm{H}$ NMR (600 MHz, CDCl 3$) ;{ }^{13} \mathrm{C}$ NMR (150 MHz, CDCl $)$

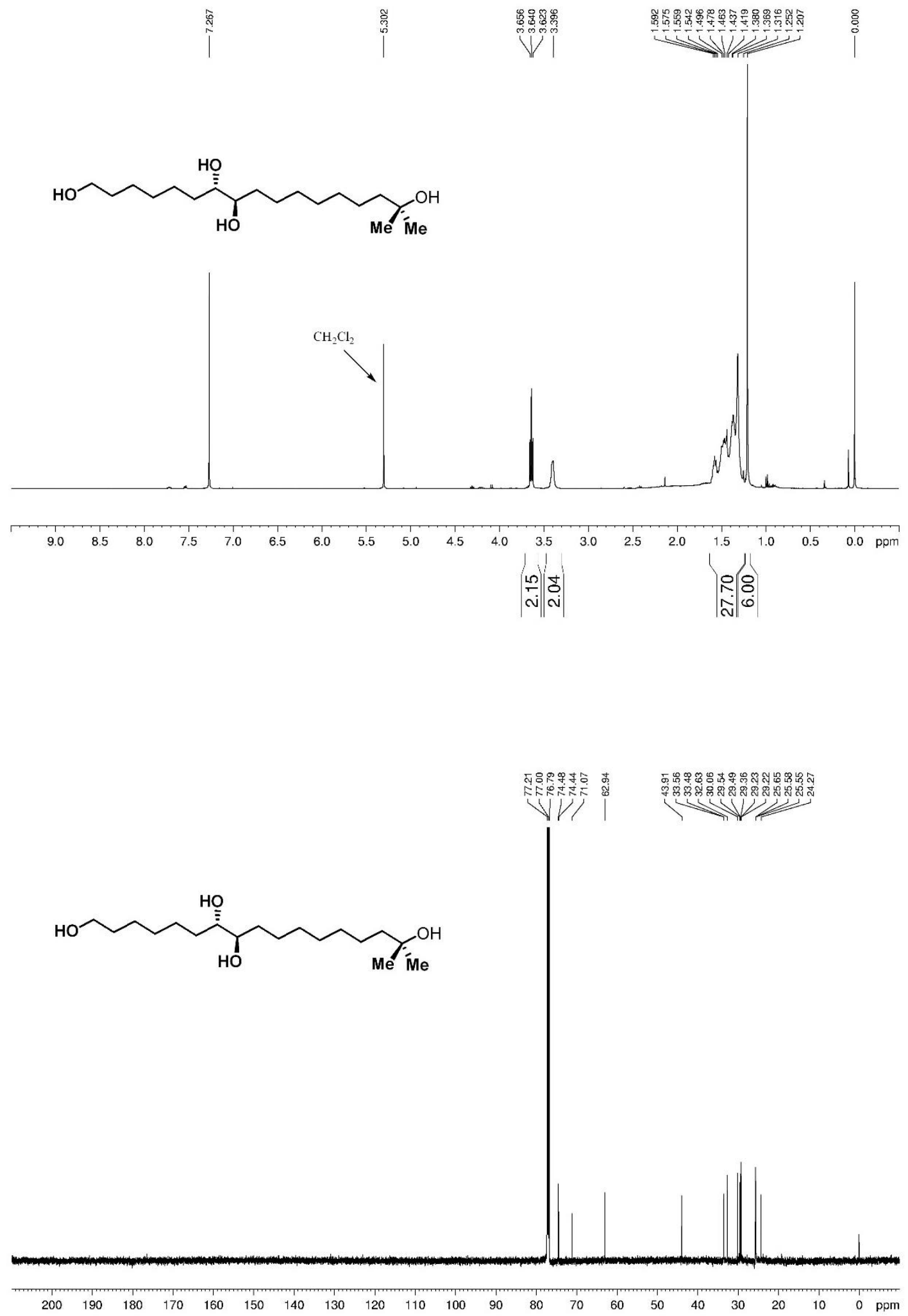


1ae; ${ }^{1} \mathrm{H}$ NMR (600 MHz, CDCl $) ;{ }^{13} \mathrm{C}$ NMR (150 MHz, CDCl $)$
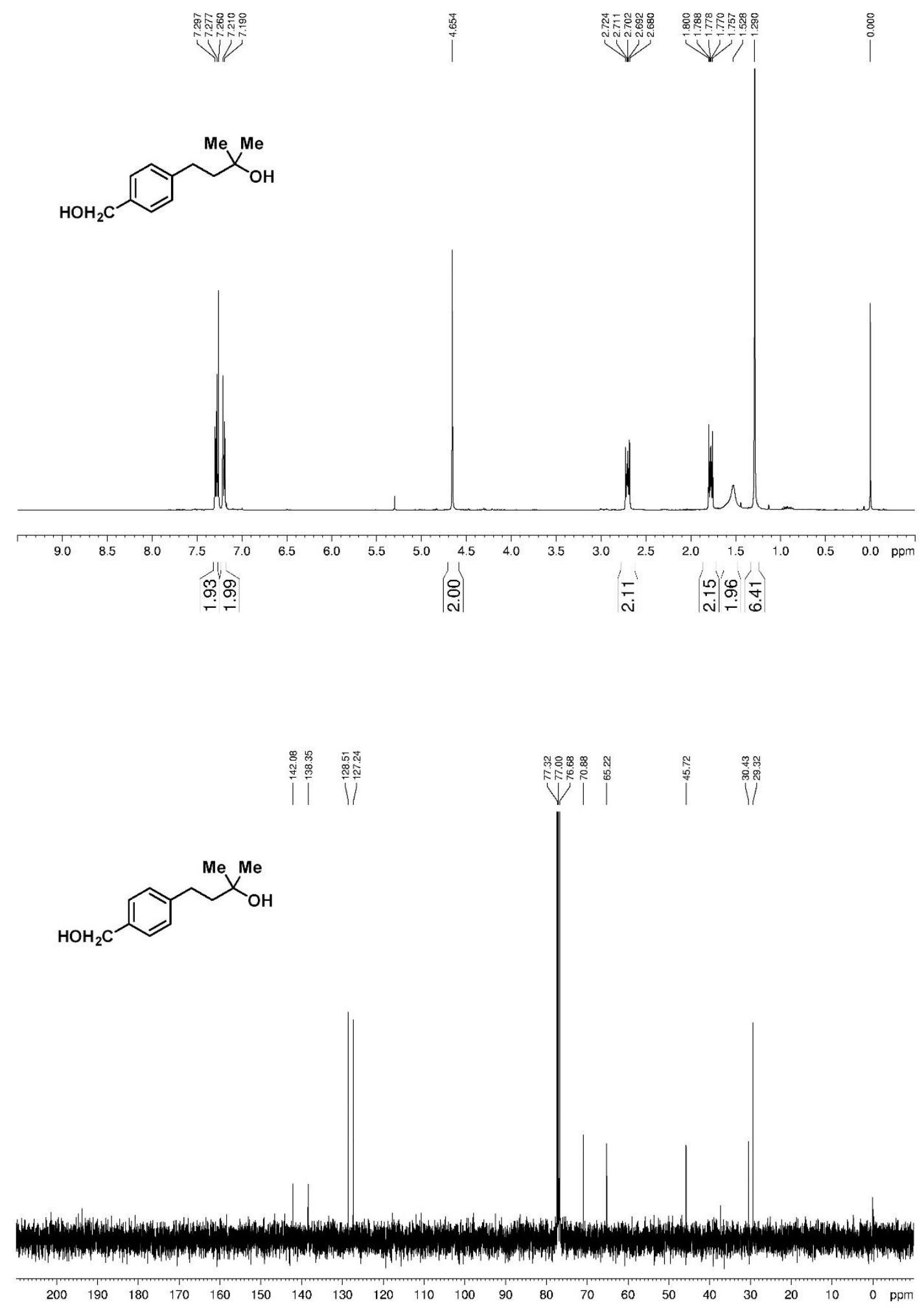
1af; ${ }^{1} \mathrm{H}$ NMR (600 MHz, CDCl$\left.) ;{ }^{13} \mathrm{C} \mathrm{NMR} \mathrm{(150} \mathrm{MHz,} \mathrm{CDCl} 3\right)$

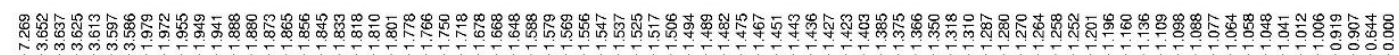

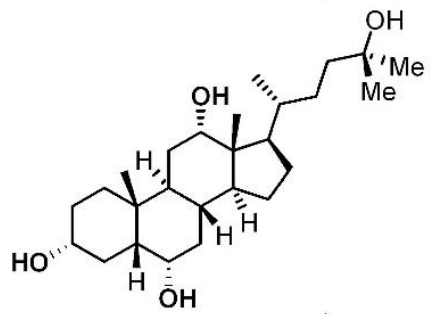

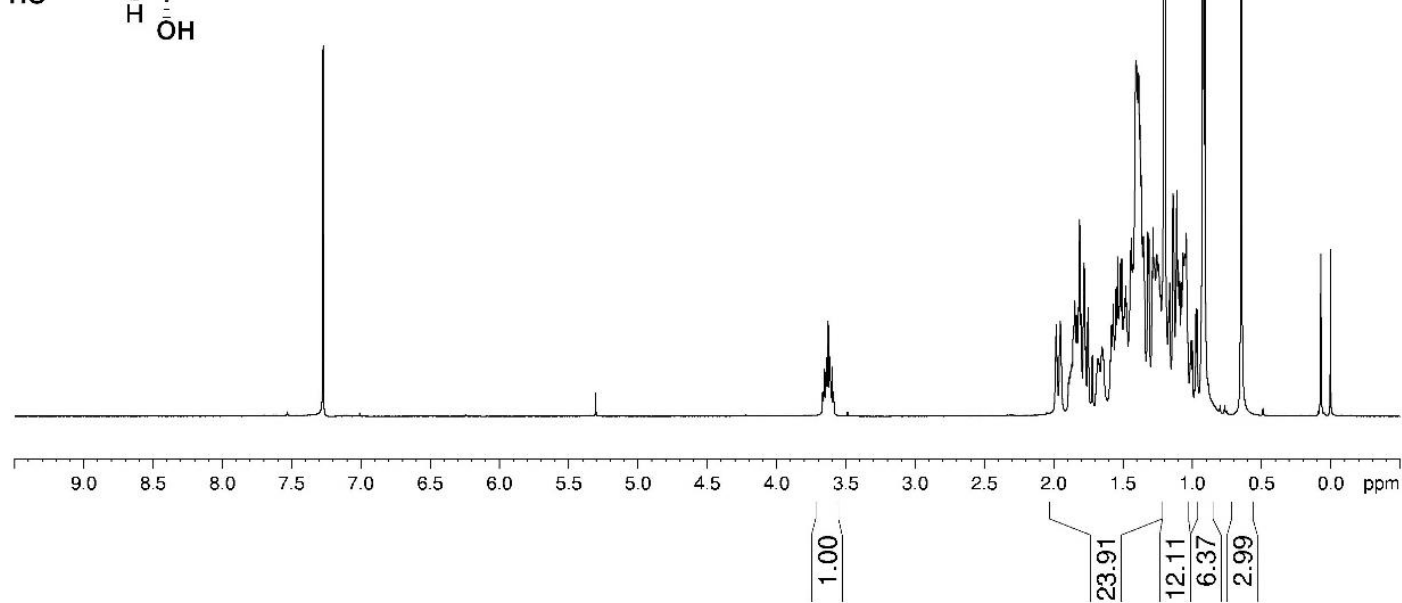

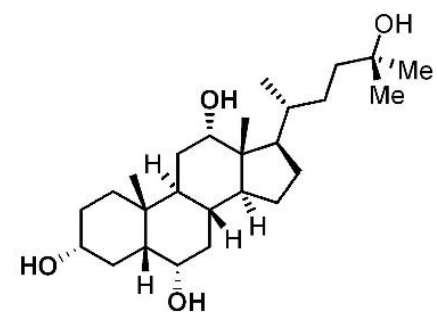

우요요

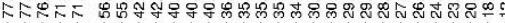
$V$ W

${ }^{\mathrm{H}} \mathrm{O} \mathrm{H}$ 
2h; ${ }^{1} \mathrm{H}$ NMR (400 MHz, CDCl$\left.) ;{ }^{13} \mathrm{C} \mathrm{NMR} \mathrm{(100} \mathrm{MHz,} \mathrm{CDCl}\right)$

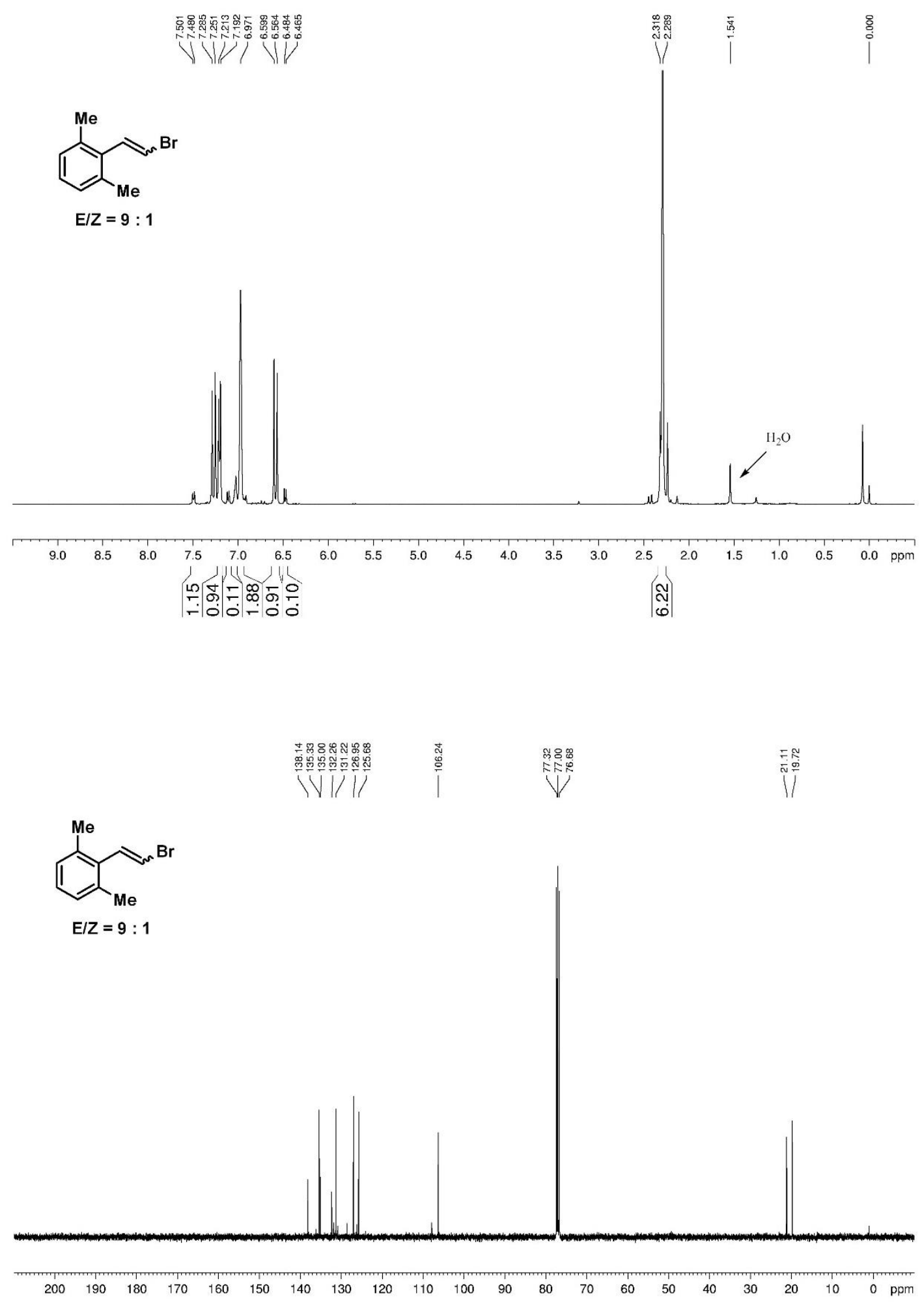


2i; ${ }^{1} \mathrm{H}$ NMR (400 MHz, $\left.\mathrm{CDCl}_{3}\right) ;{ }^{13} \mathrm{C}$ NMR (100 $\left.\mathrm{MHz}, \mathrm{CDCl}_{3}\right)$
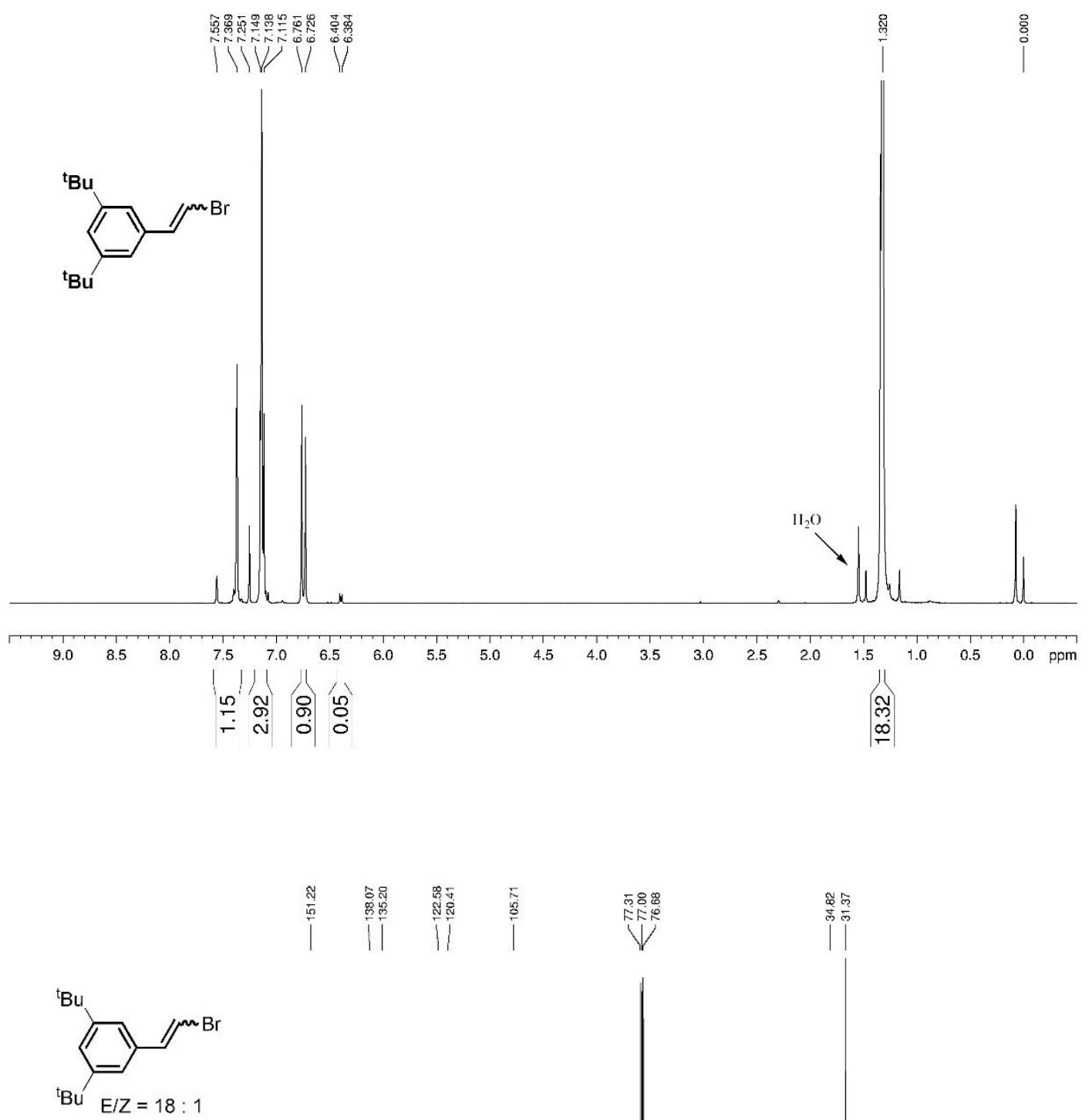
2j; ${ }^{1} \mathrm{H}$ NMR (400 MHz, $\left.\mathrm{CDCl}_{3}\right) ;{ }^{13} \mathrm{C}$ NMR (100 MHz, $\left.\mathrm{CDCl}_{3}\right)$
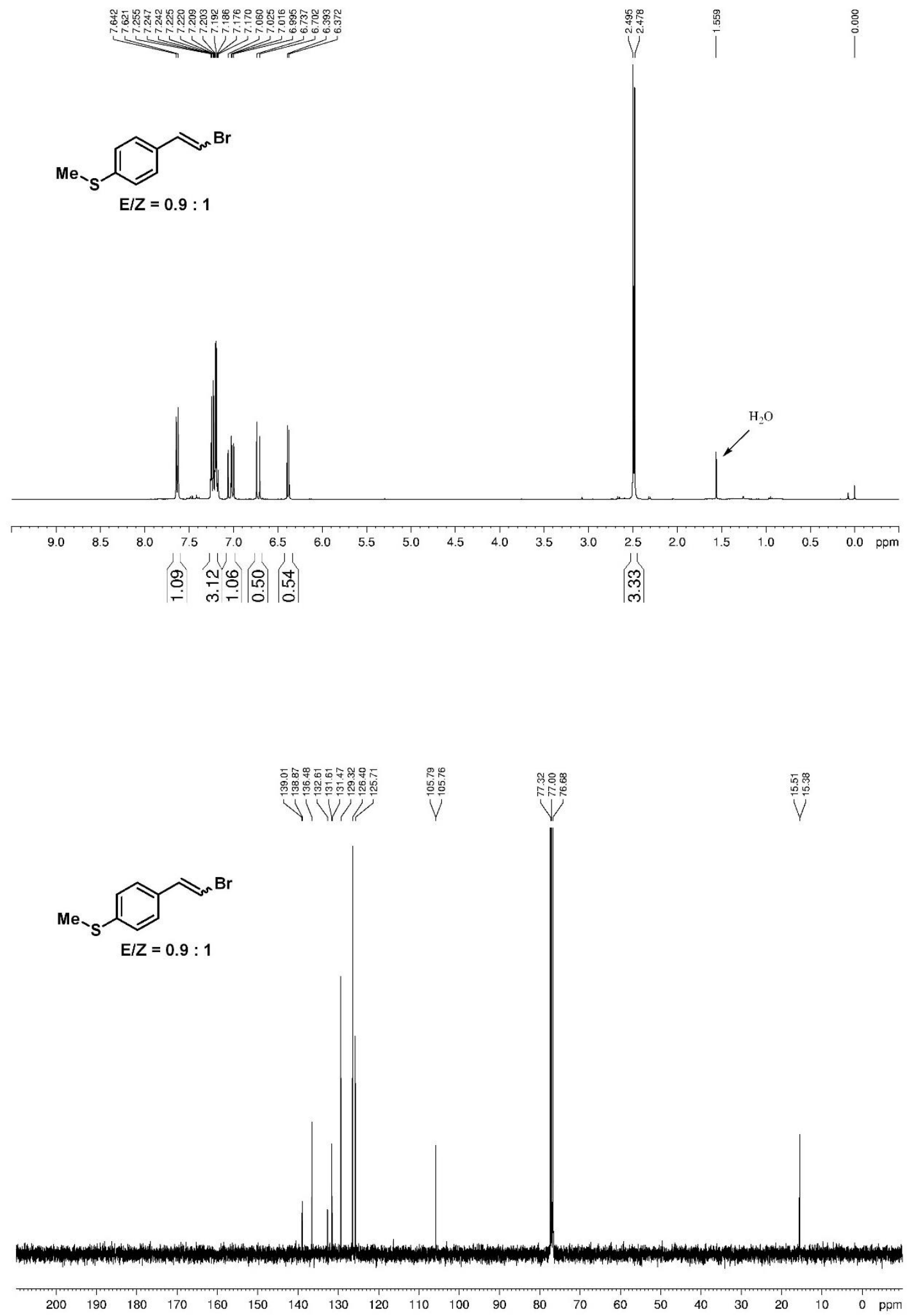
2k; ${ }^{1} \mathrm{H}$ NMR (600 MHz, CDCl $\left.) ;{ }^{13} \mathrm{C} \mathrm{NMR} \mathrm{(150} \mathrm{MHz,} \mathrm{CDCl}\right)$

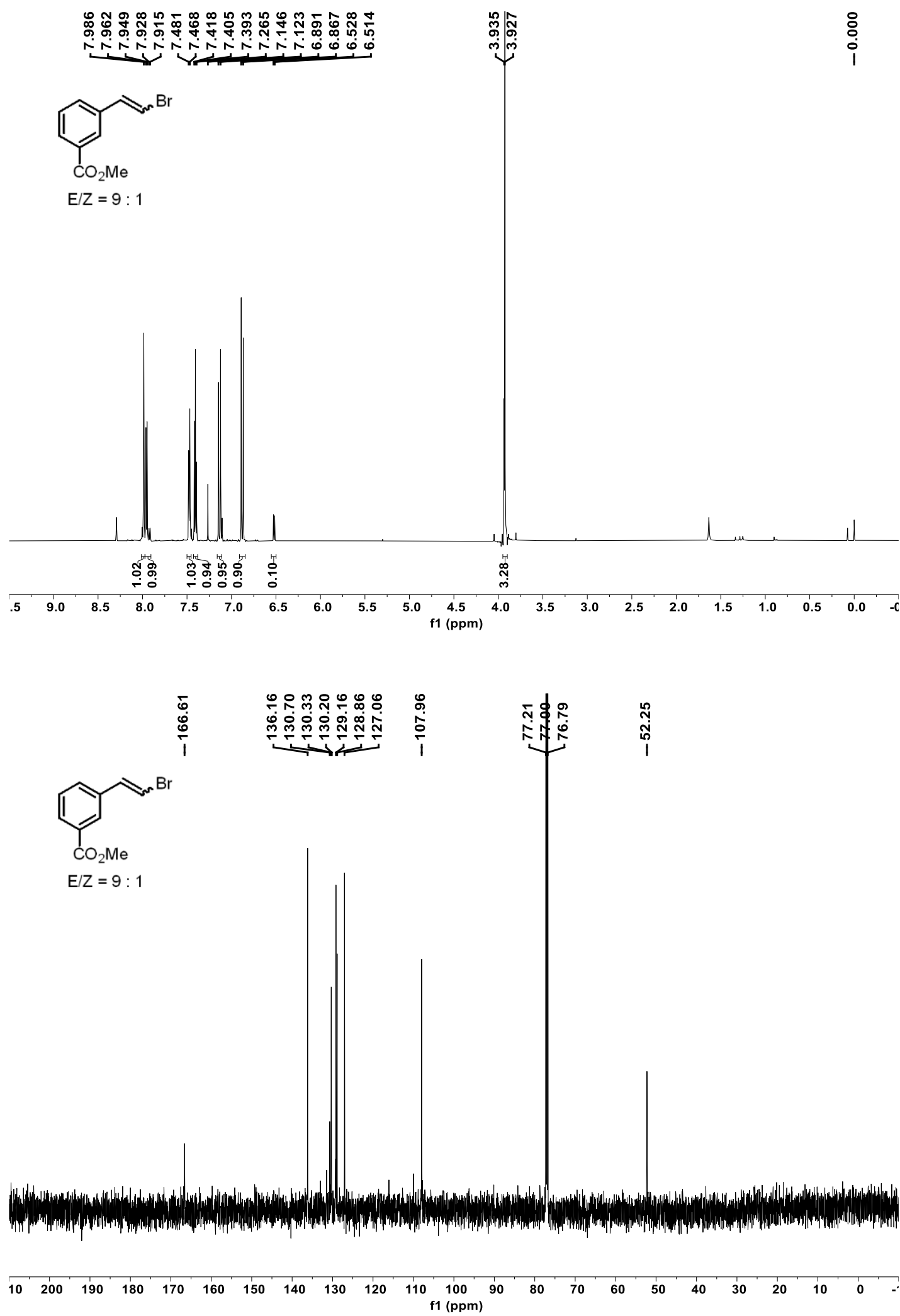


2I; ${ }^{1} \mathrm{H}$ NMR (400 MHz, $\left.\mathrm{CDCl}_{3}\right) ;{ }^{13} \mathrm{C}$ NMR (100 $\left.\mathrm{MHz}, \mathrm{CDCl}_{3}\right)$

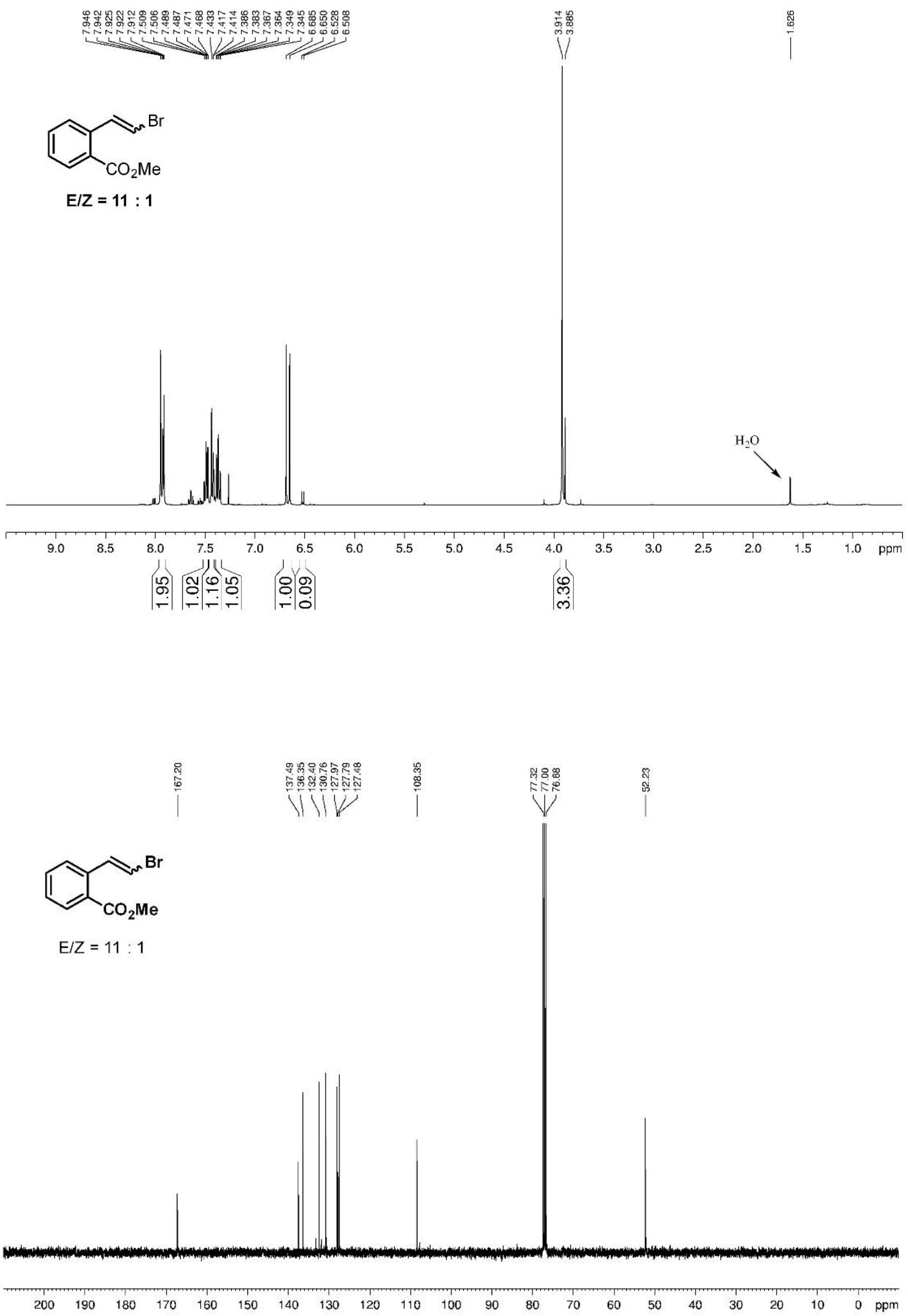


2u; ${ }^{1} \mathrm{H}$ NMR (600 MHz, CDCl $\left.) ;{ }^{13} \mathrm{C} \mathrm{NMR} \mathrm{(150} \mathrm{MHz,} \mathrm{CDCl}\right)$
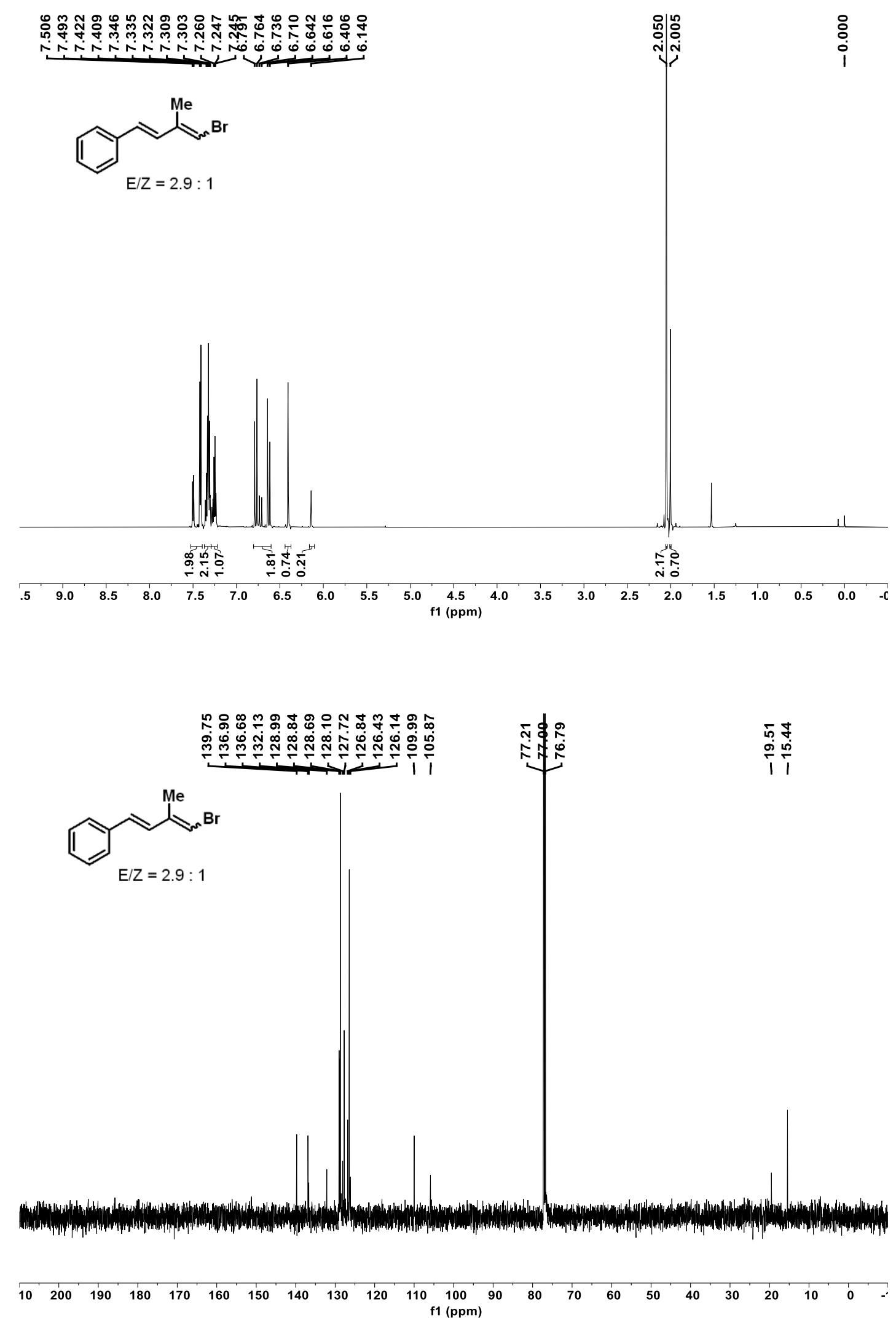
2w; ${ }^{1} \mathrm{H}$ NMR (600 MHz, CDCl $) ;{ }^{13} \mathrm{C}$ NMR (150 MHz, CDCl $)$

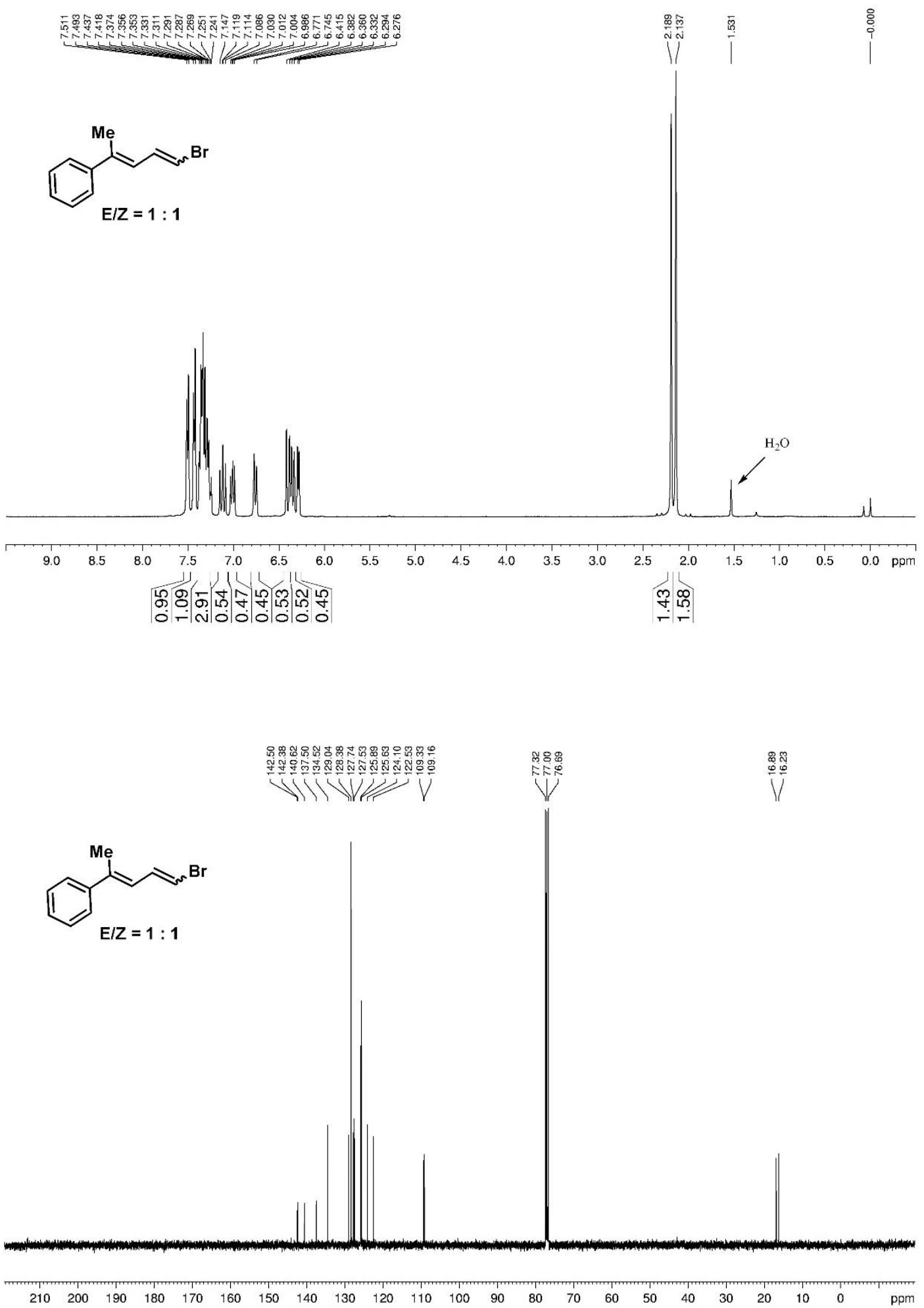


2ac; ${ }^{1} \mathrm{H}$ NMR (400 MHz, CDCl $) ;{ }^{13} \mathrm{C}$ NMR (100 MHz, CDCl $)$

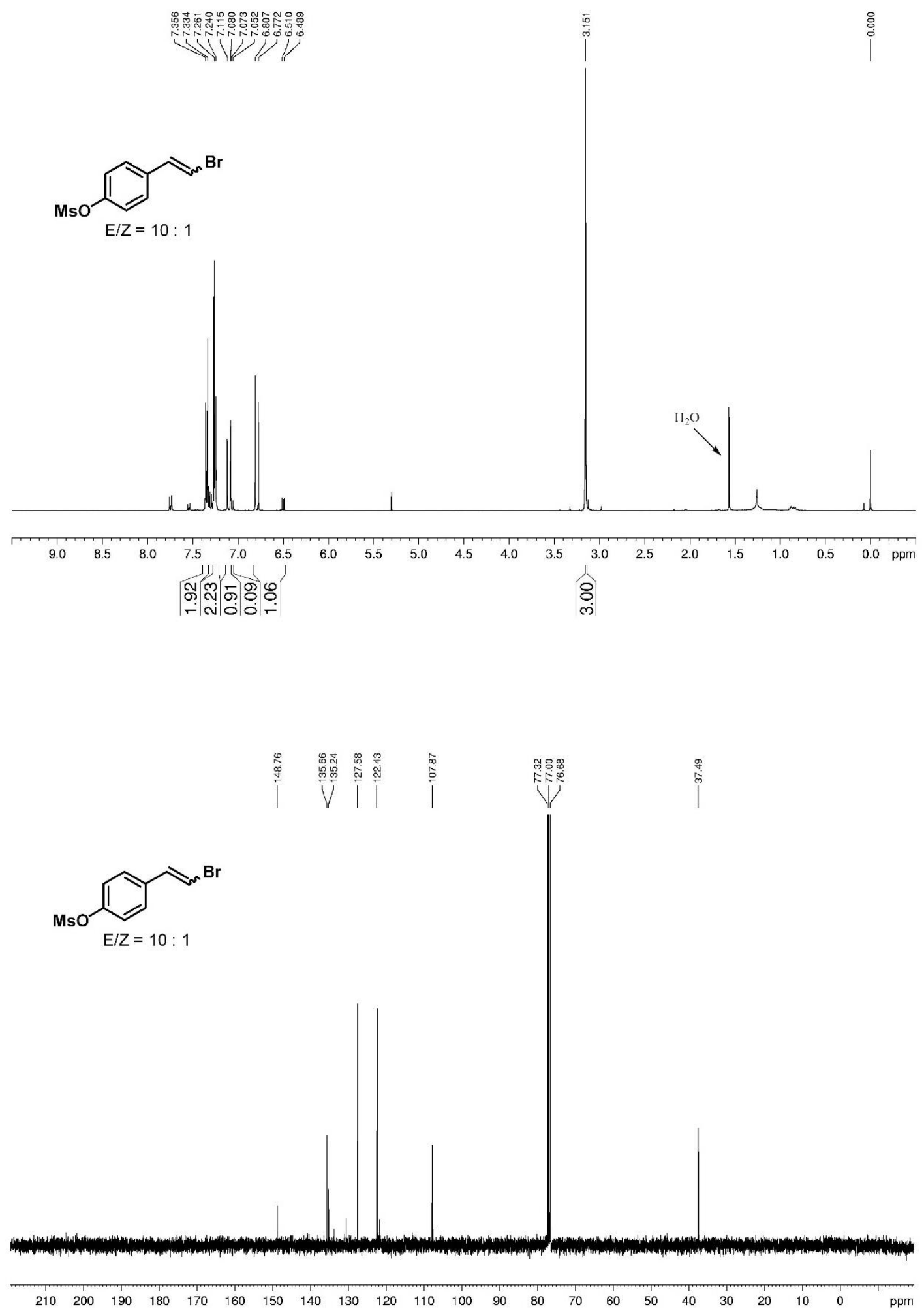


2ad; ${ }^{1} \mathrm{H}$ NMR (600 MHz, $\left.\mathrm{CDCl}_{3}\right) ;{ }^{13} \mathrm{C}$ NMR (150 MHz, $\left.\mathrm{CDCl}_{3}\right)$
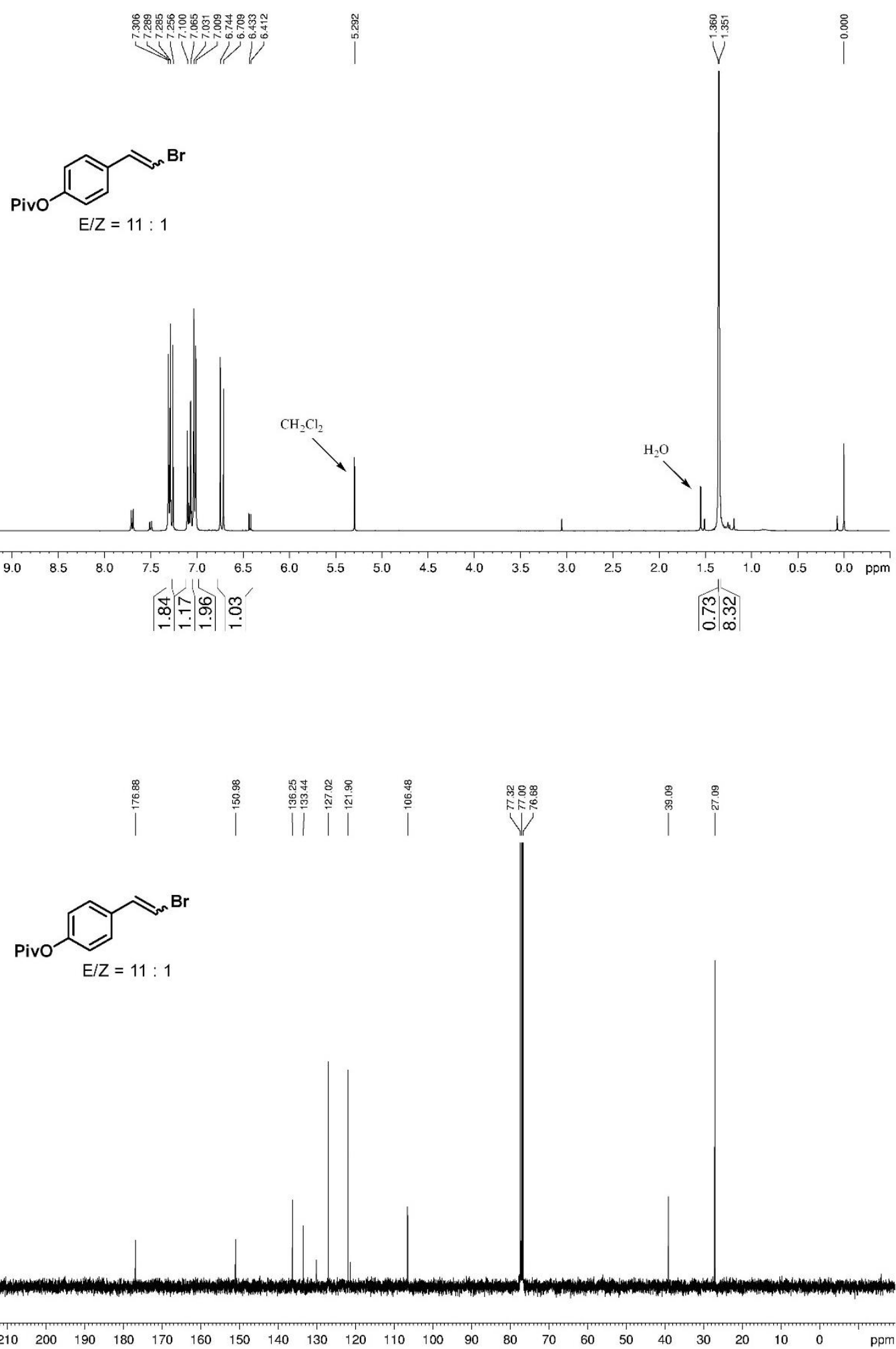
2ae; ${ }^{1} \mathrm{H}$ NMR (600 MHz, CDCl $) ;{ }^{13} \mathrm{C}$ NMR (150 MHz, CDCl $)$

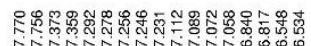

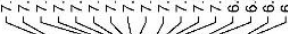
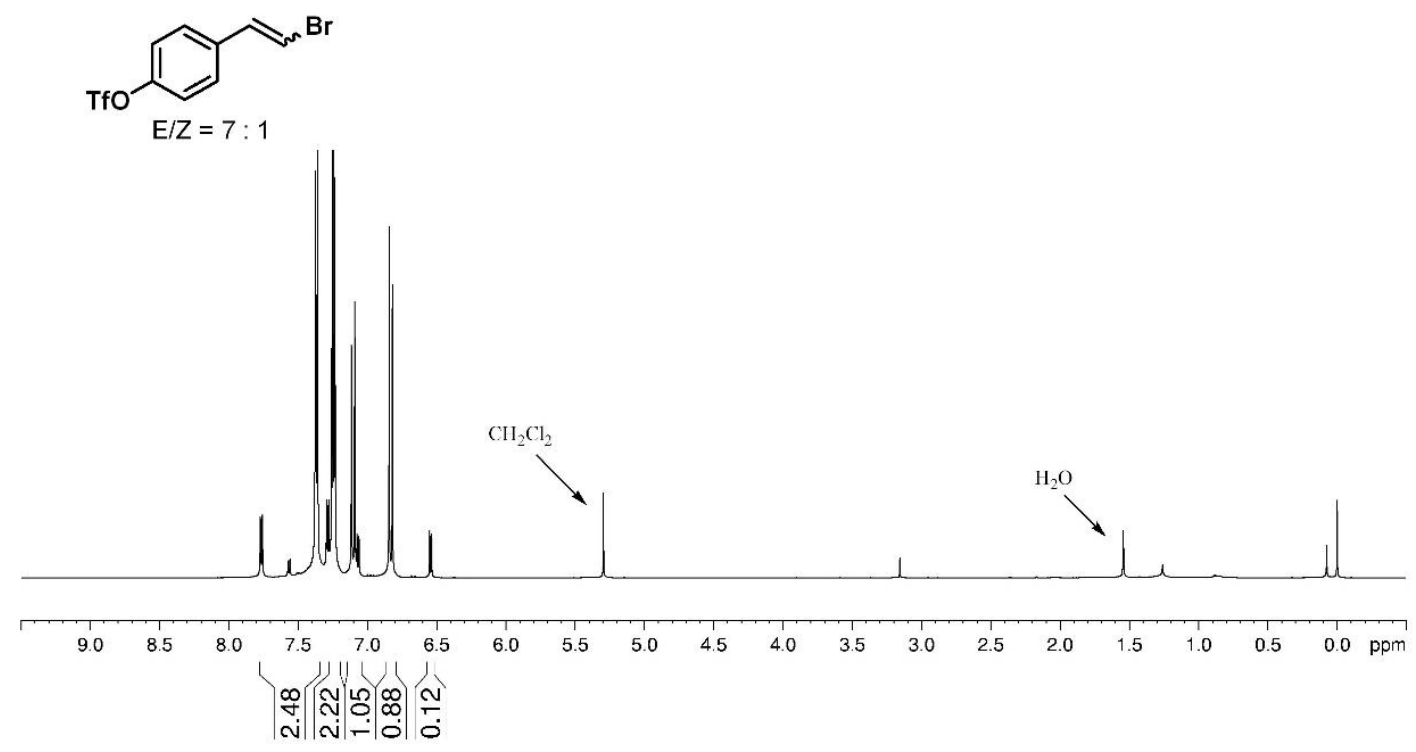

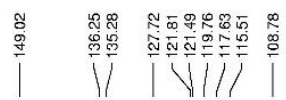

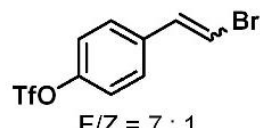

$E / Z=7: 1$

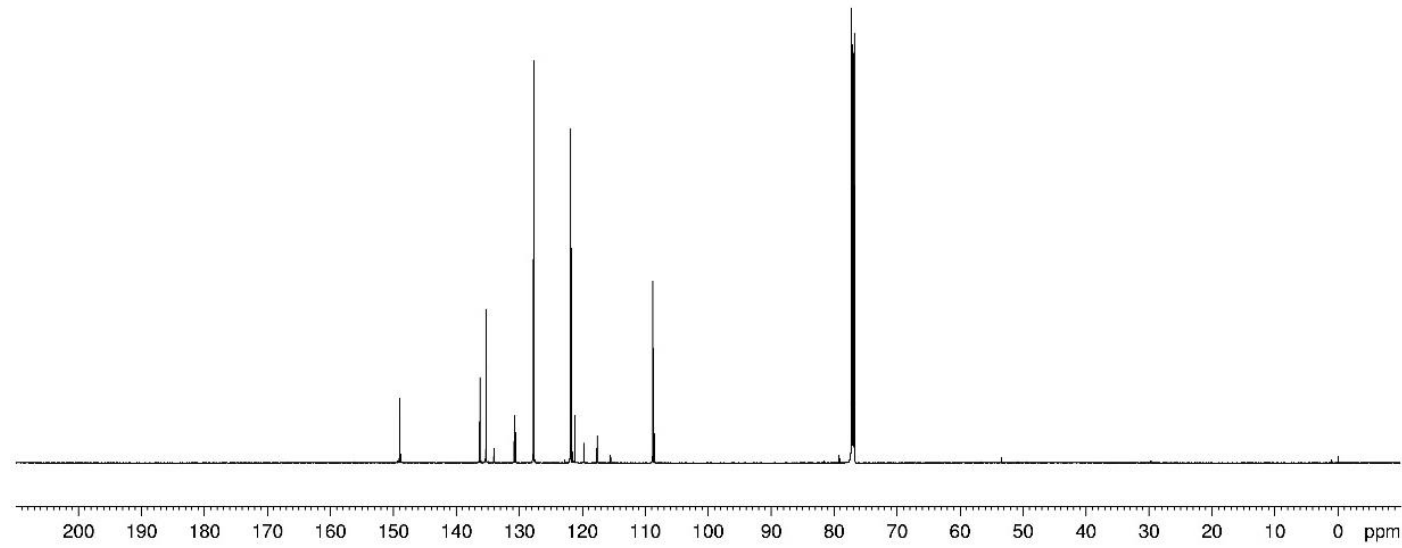


2ae; ${ }^{19}$ F NMR (376 MHz, CDCl3)

ฟั<smiles>Oc1ccc(C=CBr)cc1</smiles>

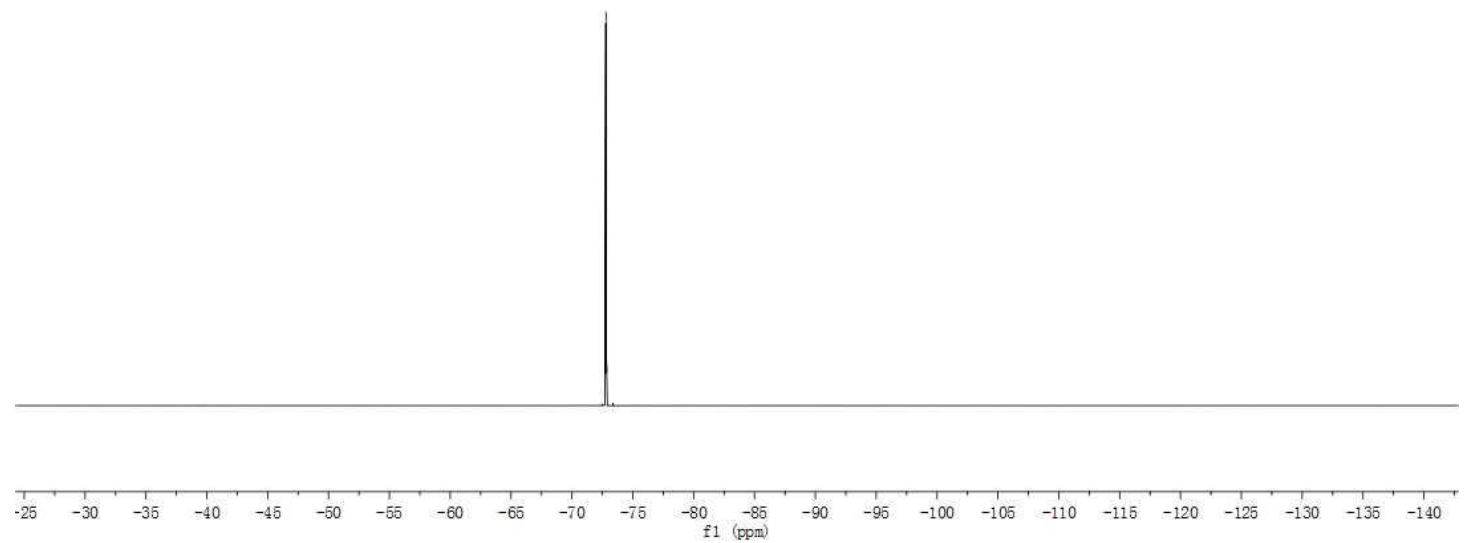


4; ${ }^{1} \mathrm{H}$ NMR (400 MHz, CDCl3); ${ }^{13} \mathrm{C}$ NMR (100 MHz, CDCl $)$

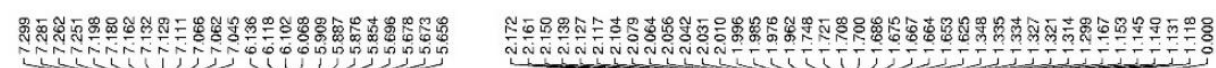

$10 m_{B r}$

$E / Z=1: 4$

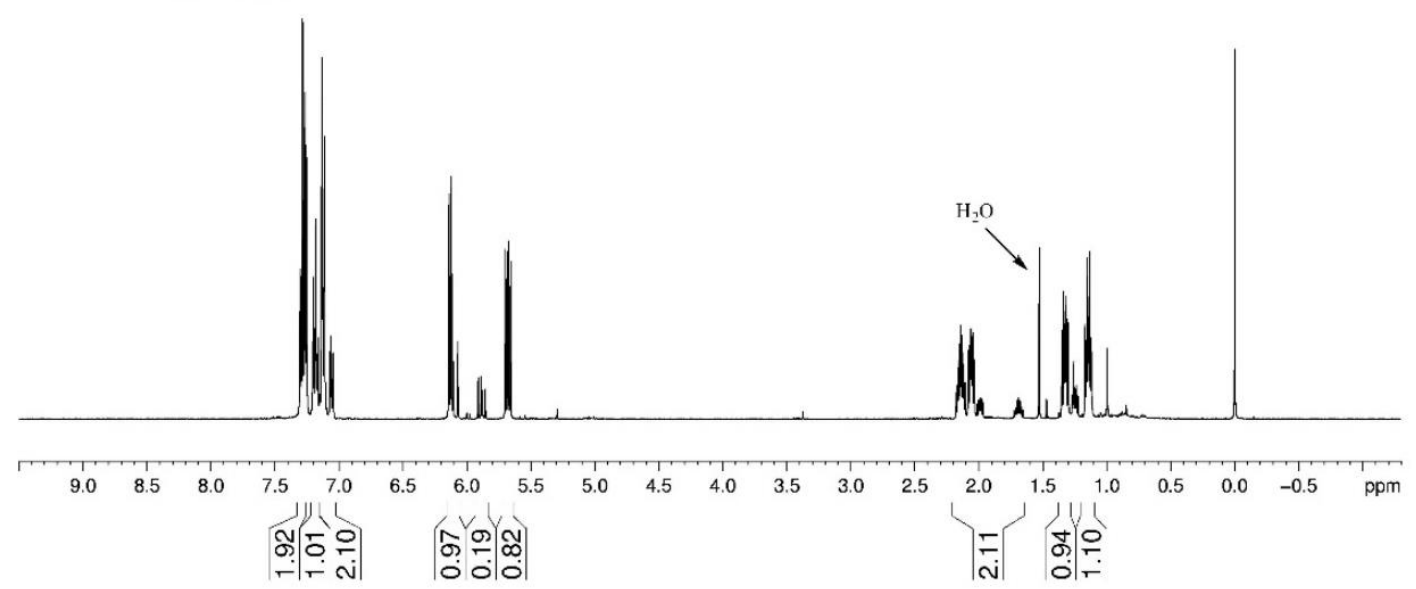

||<smiles>BrC=CC1CC1c1ccccc1</smiles>

$E / Z=1: 4$

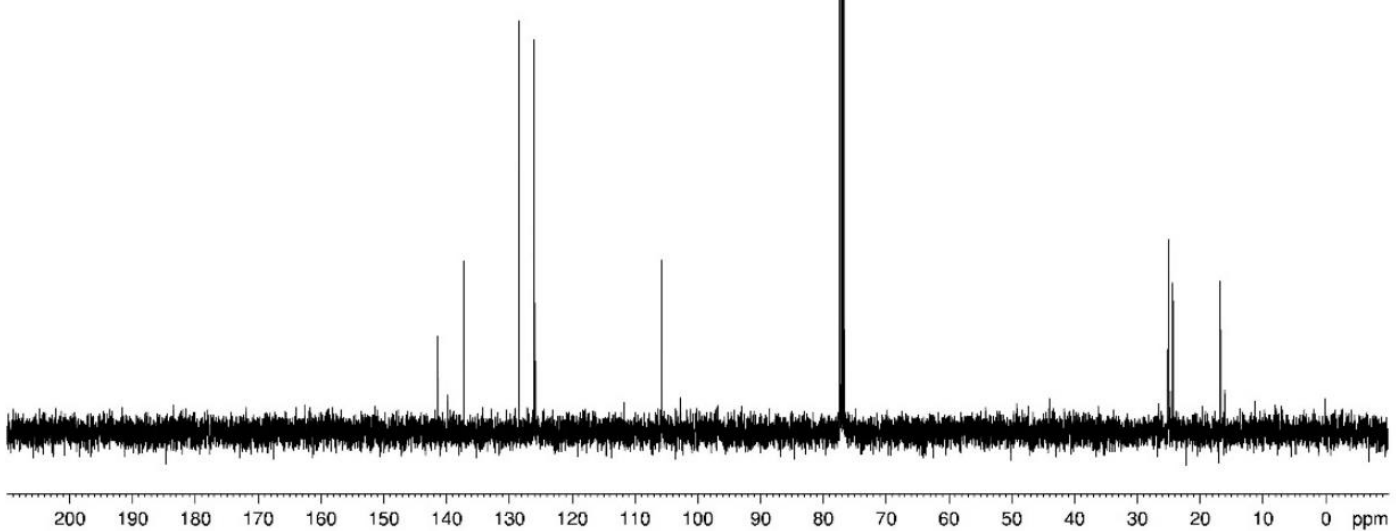


8; ${ }^{1} \mathrm{H}$ NMR (400 MHz, $\left.\mathrm{CDCl}_{3}\right) ;{ }^{13} \mathrm{C}$ NMR (100 $\left.\mathrm{MHz}, \mathrm{CDCl}_{3}\right)$

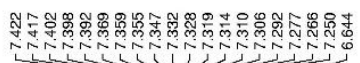

$\mid$

i<smiles>BrC=C(CBr)c1ccccc1</smiles>
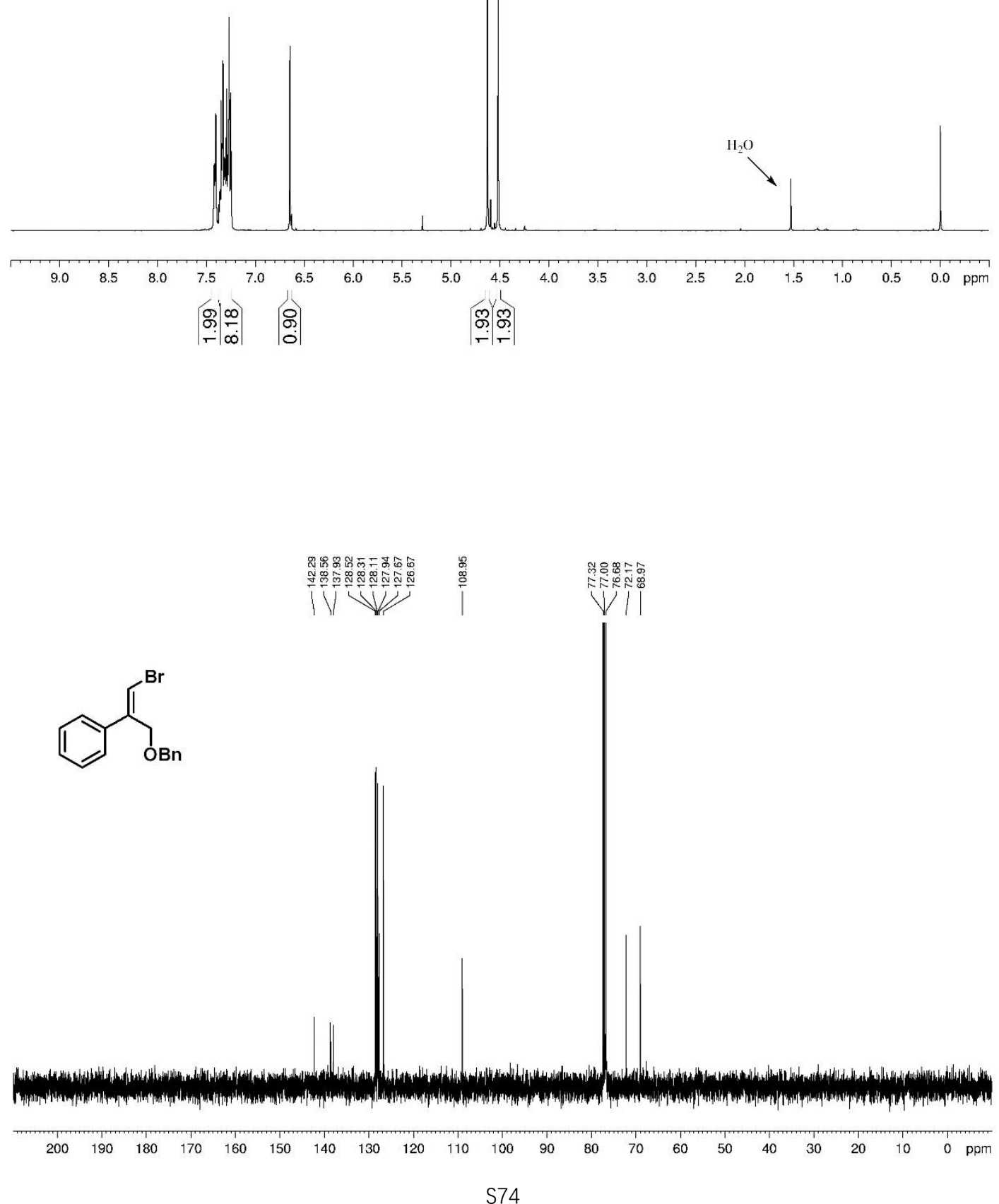
3a; ${ }^{1} \mathrm{H}$ NMR (400 MHz, CDCl3); ${ }^{13} \mathrm{C}$ NMR (100 MHz, CDCl $)$

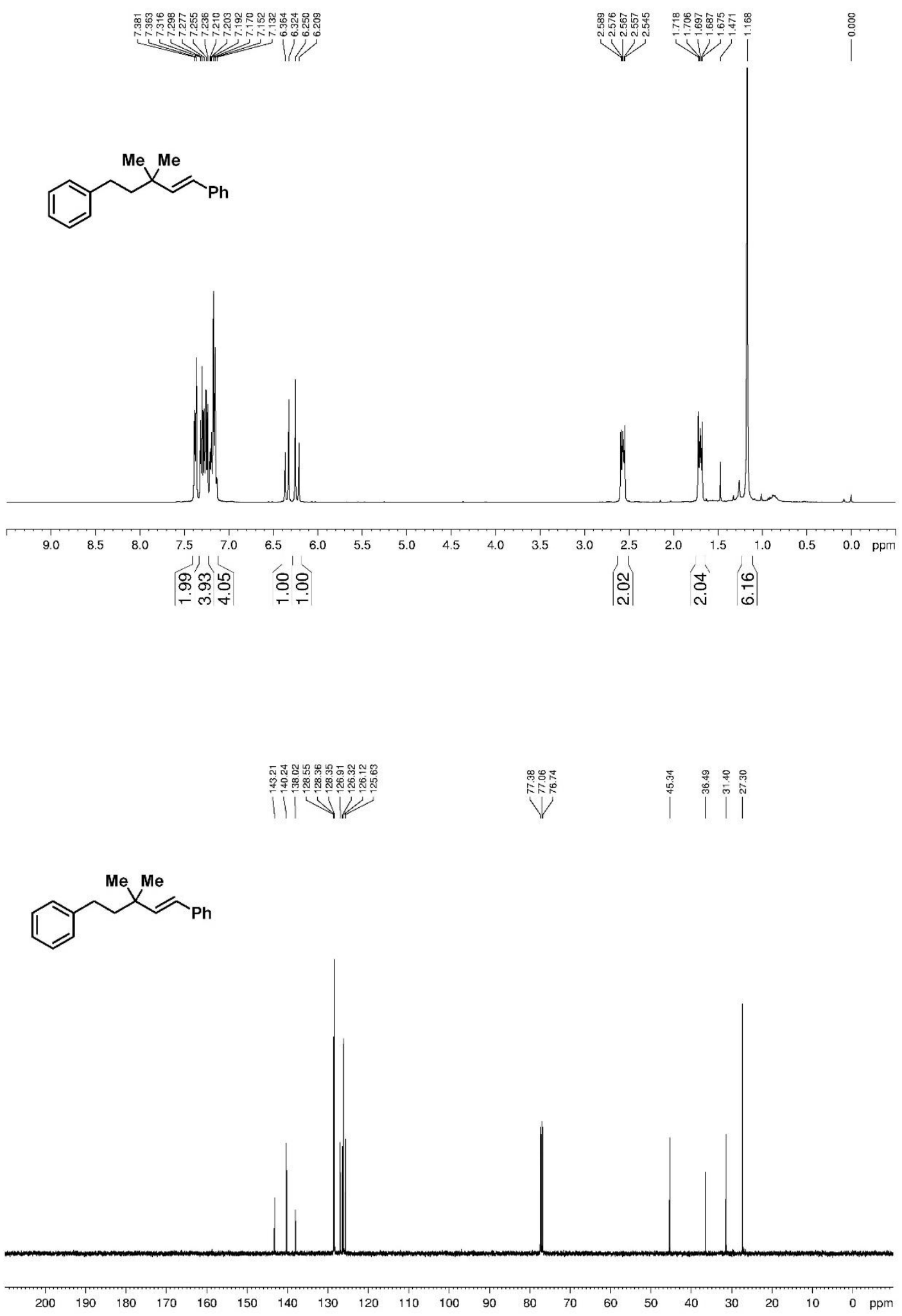


3b; ${ }^{1} \mathrm{H}$ NMR (400 MHz, CDCl$\left.) ;{ }^{13} \mathrm{C} \mathrm{NMR} \mathrm{(100} \mathrm{MHz,} \mathrm{CDCl}\right)$
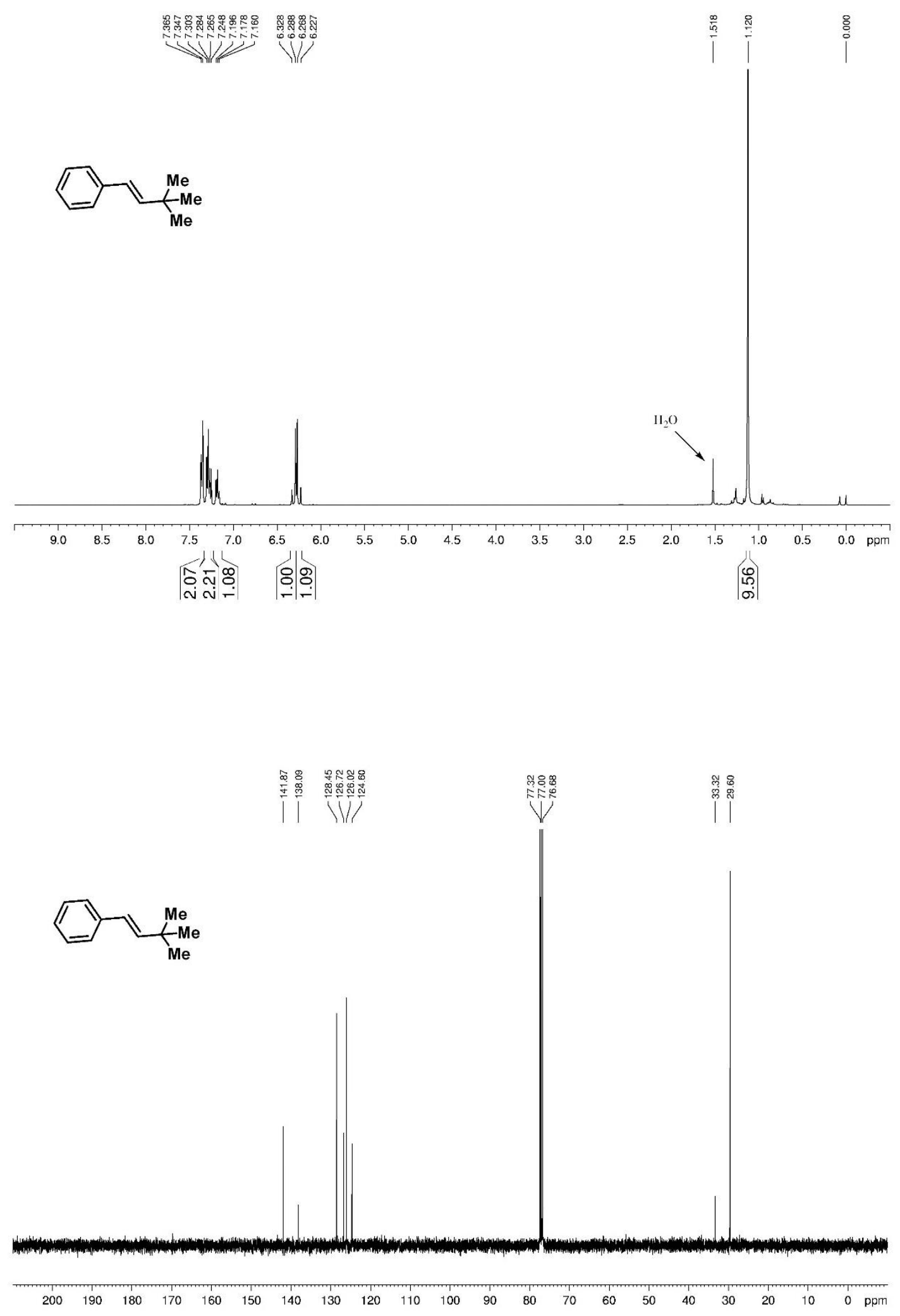
3c; ${ }^{1} \mathrm{H}$ NMR (400 MHz, $\left.\mathrm{CDCl}_{3}\right) ;{ }^{13} \mathrm{C}$ NMR (100 MHz, $\left.\mathrm{CDCl}_{3}\right)$

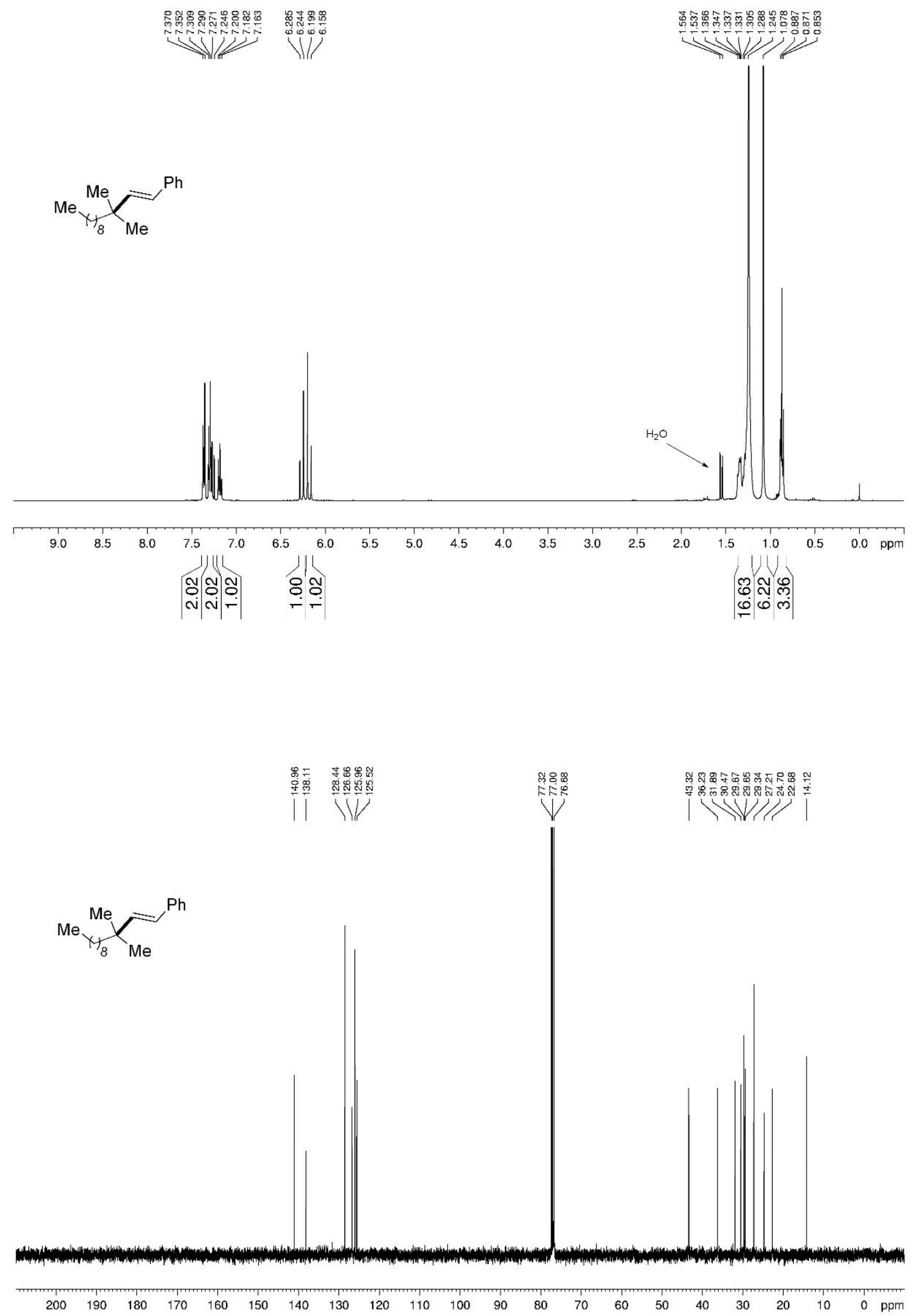


3d; ${ }^{1} \mathrm{H}$ NMR (400 MHz, CDCl $\left.) ;{ }^{13} \mathrm{C} \mathrm{NMR} \mathrm{(100} \mathrm{MHz}^{\mathrm{CDCl}} 3\right)$

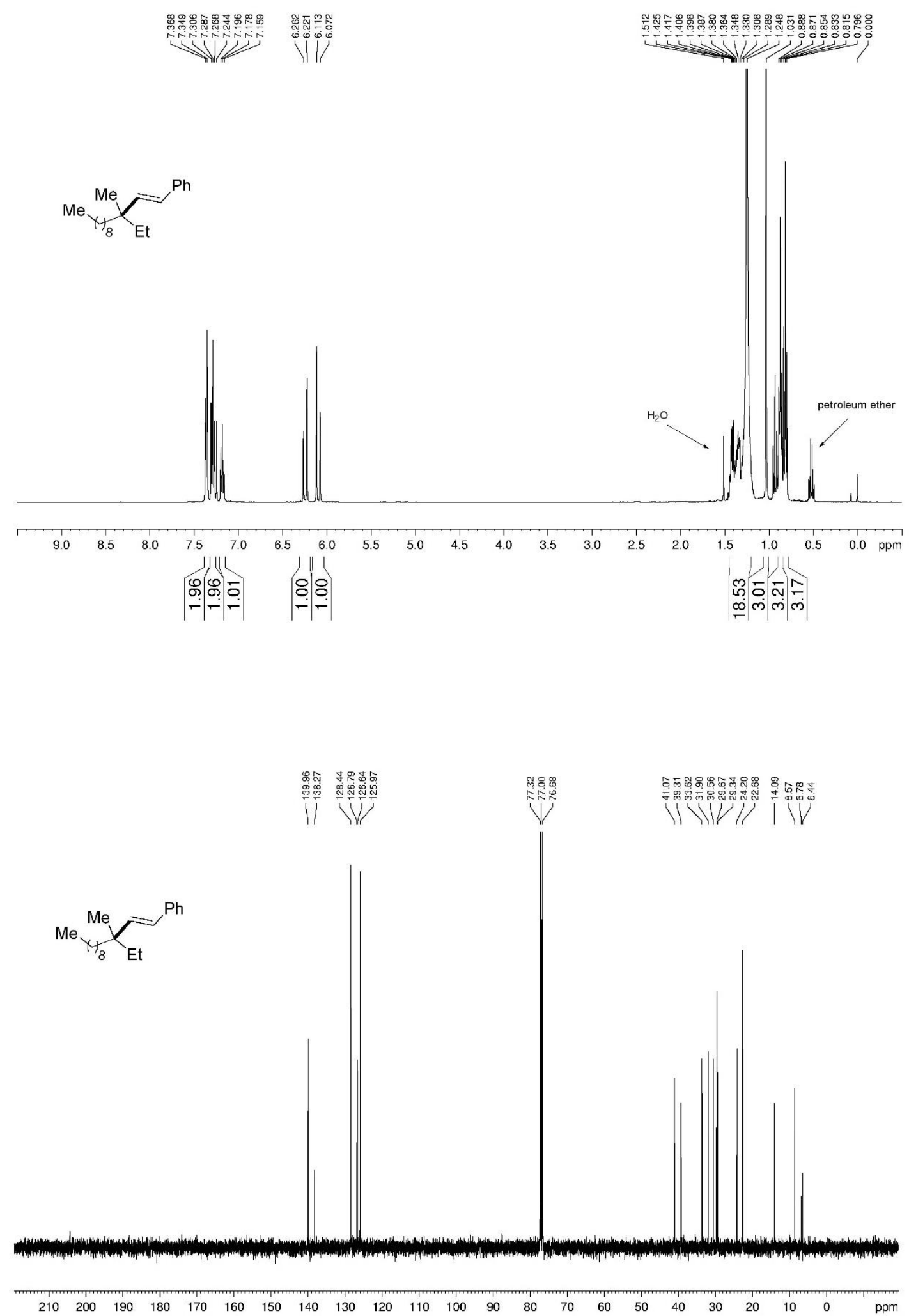


3e; ${ }^{1} \mathrm{H}$ NMR (400 MHz, $\left.\mathrm{CDCl}_{3}\right) ;{ }^{13} \mathrm{C}$ NMR (100 MHz, $\left.\mathrm{CDCl}_{3}\right)$
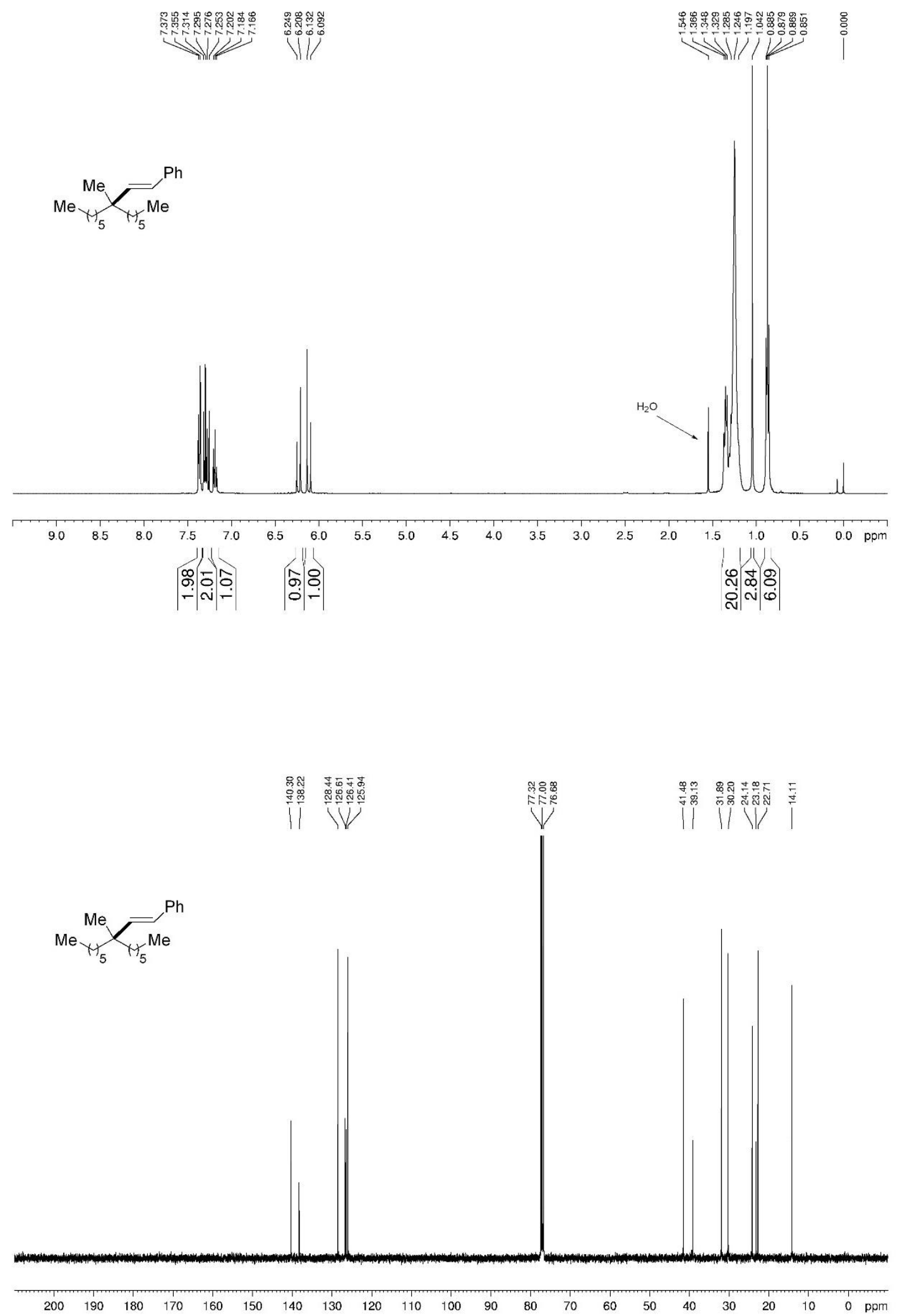
3f; ${ }^{1} \mathrm{H}$ NMR (600 MHz, CDCl 3$) ;{ }^{13} \mathrm{C}$ NMR (150 MHz, CDCl $)$
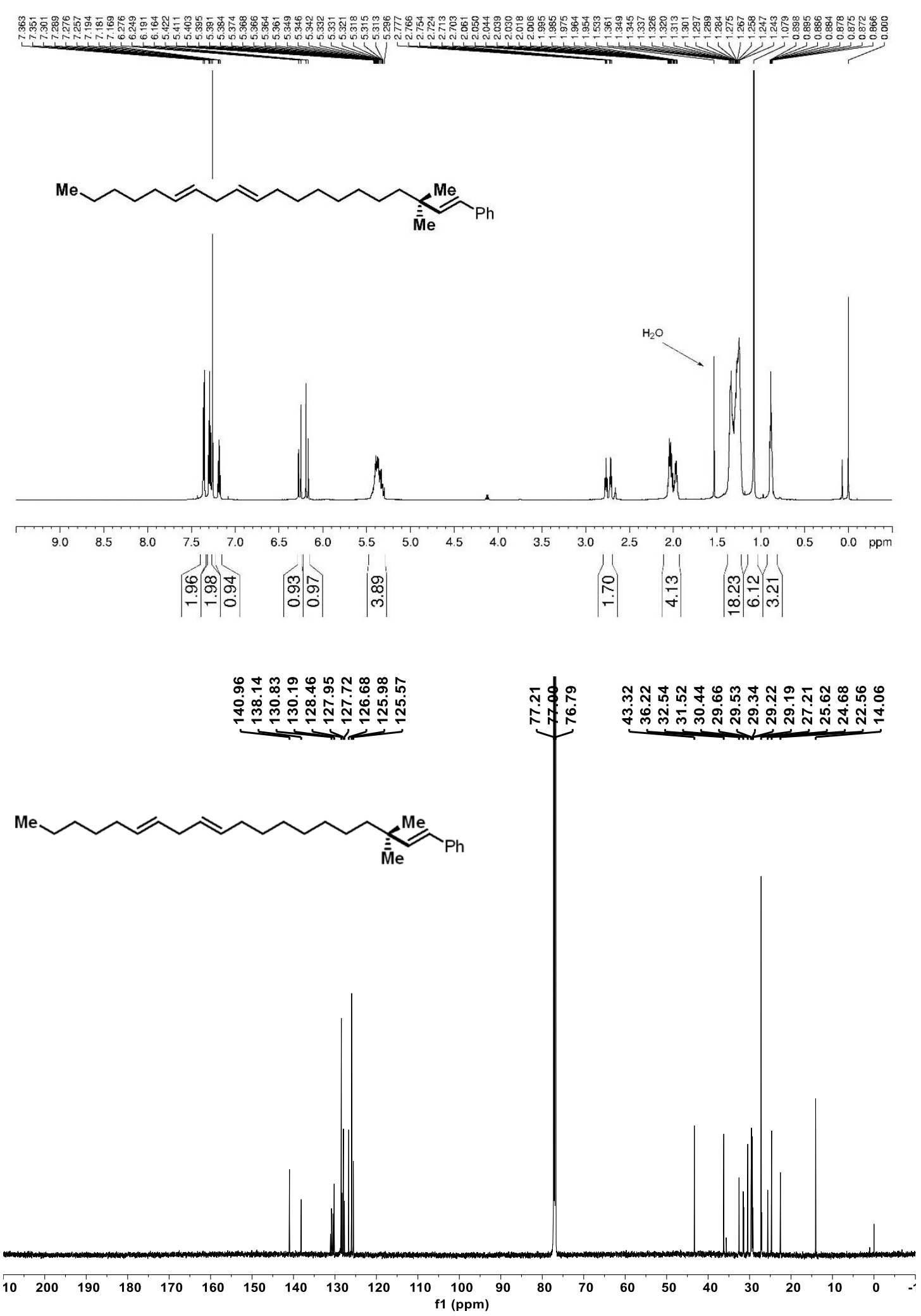
3g; ${ }^{1} \mathrm{H}$ NMR (400 MHz, $\left.\mathrm{CDCl}_{3}\right) ;{ }^{13} \mathrm{C}$ NMR (100 MHz, $\left.\mathrm{CDCl}_{3}\right)$

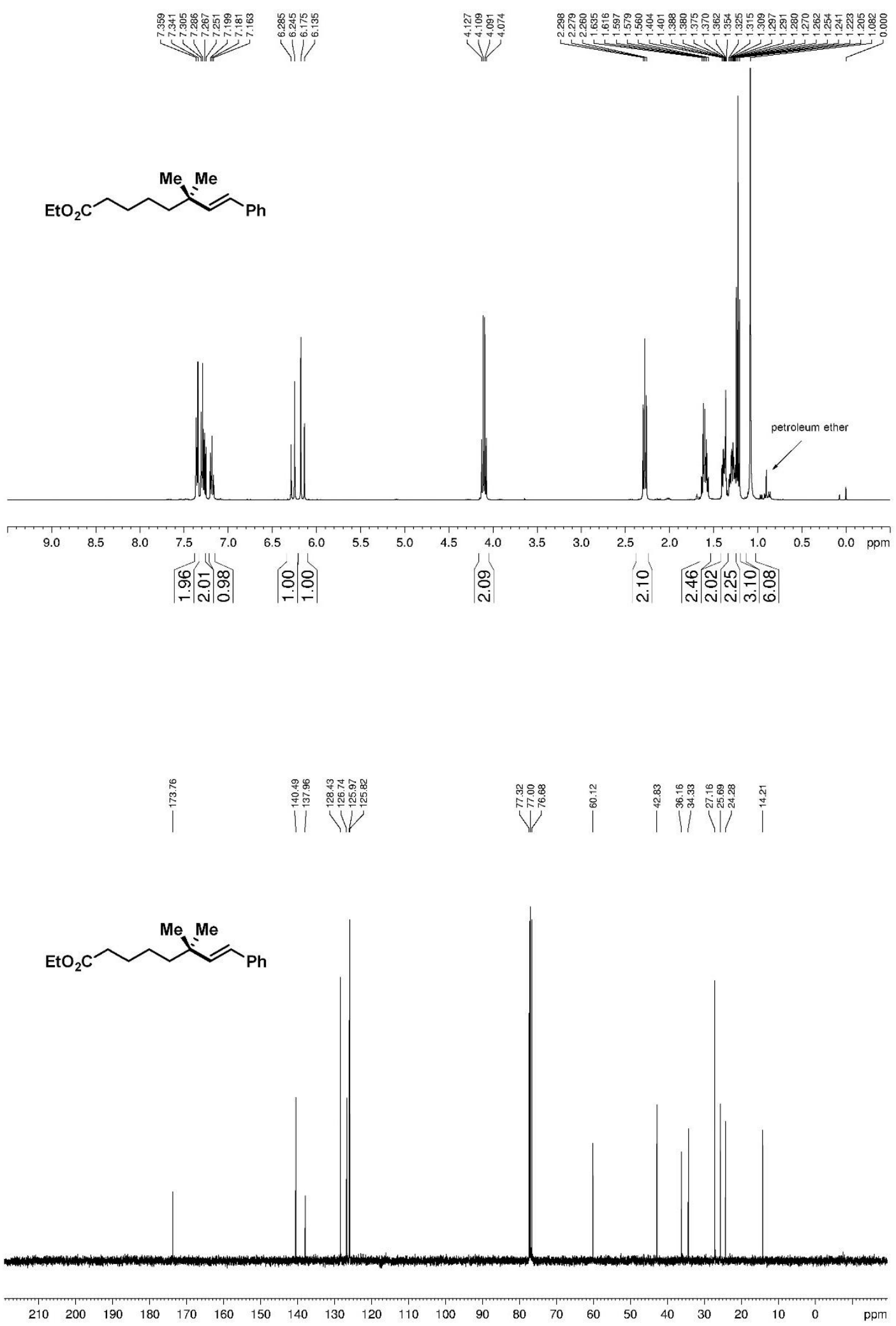


3h; ${ }^{1} \mathrm{H}$ NMR (400 MHz, CDCl $) ;{ }^{13} \mathrm{C}$ NMR (100 MHz, CDCl 3 )
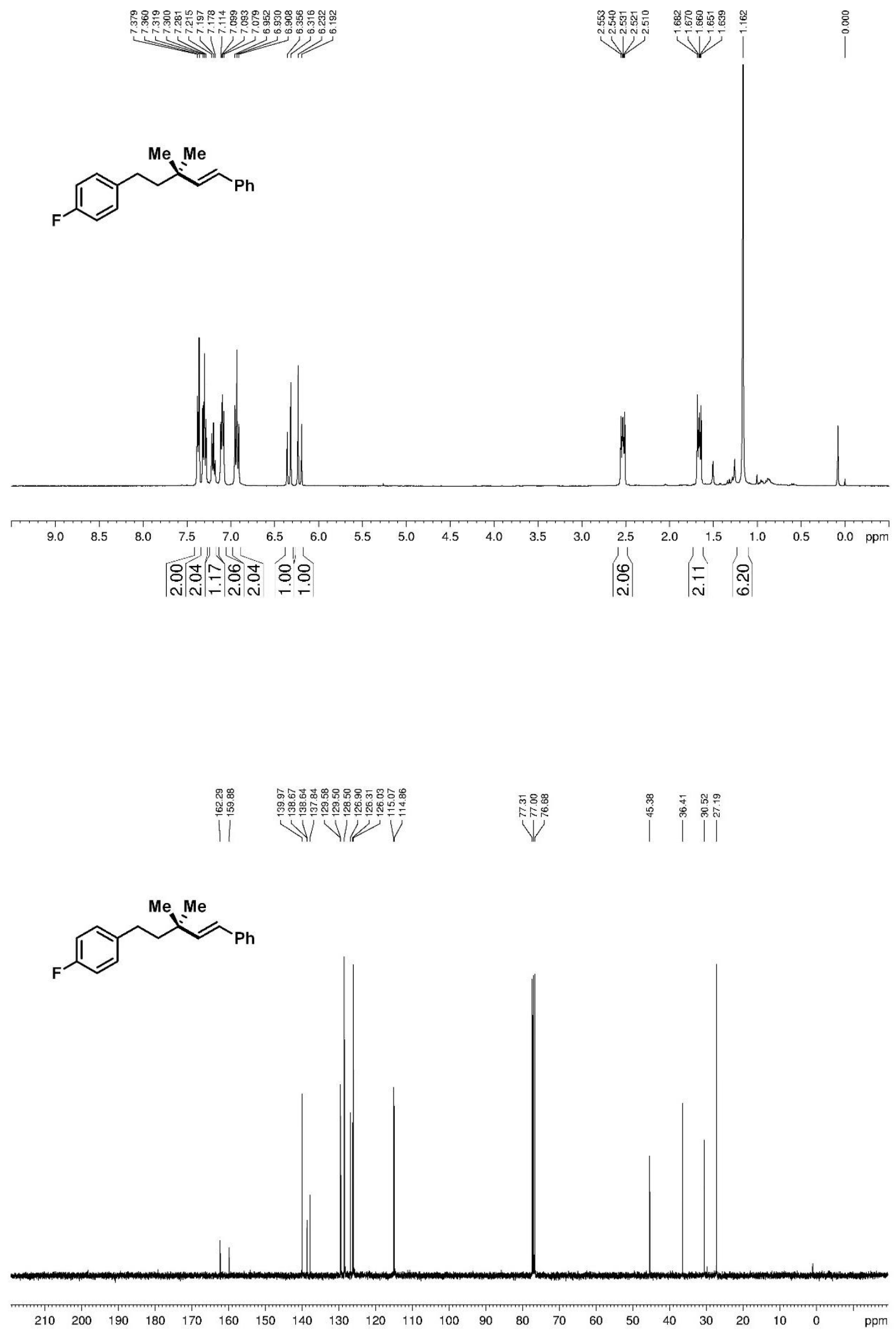

S82 
3h; ${ }^{19}$ F NMR (376 MHz, $\left.\mathrm{CDCl}_{3}\right)$

$$
\frac{\mathfrak{\infty}}{\frac{\infty}{i}}
$$<smiles>CC(C)(/C=C/c1ccccc1)CCc1ccc(F)cc1</smiles>

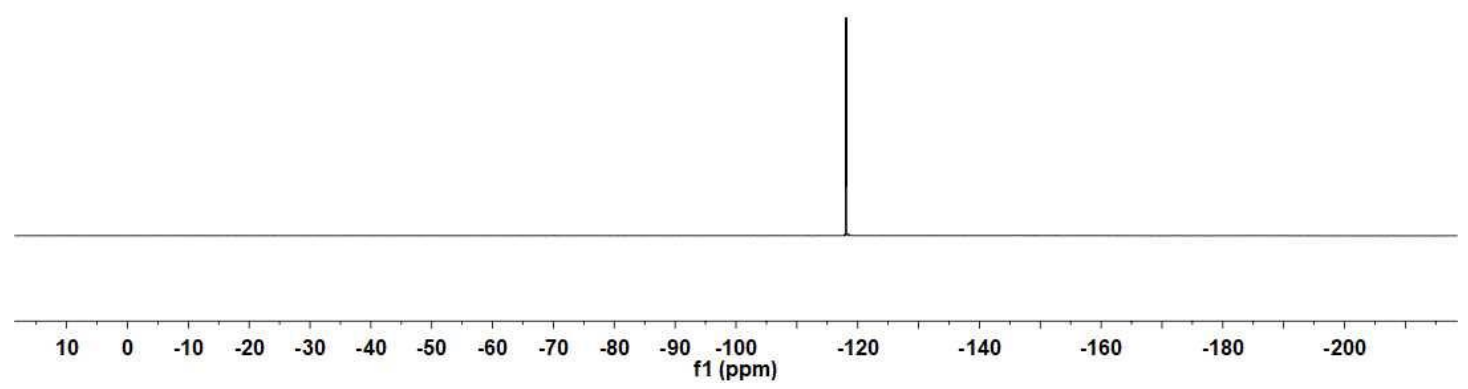


3i; ${ }^{1} \mathrm{H}$ NMR (400 MHz, $\left.\mathrm{CDCl}_{3}\right) ;{ }^{13} \mathrm{C}$ NMR (100 MHz, $\left.\mathrm{CDCl}_{3}\right)$
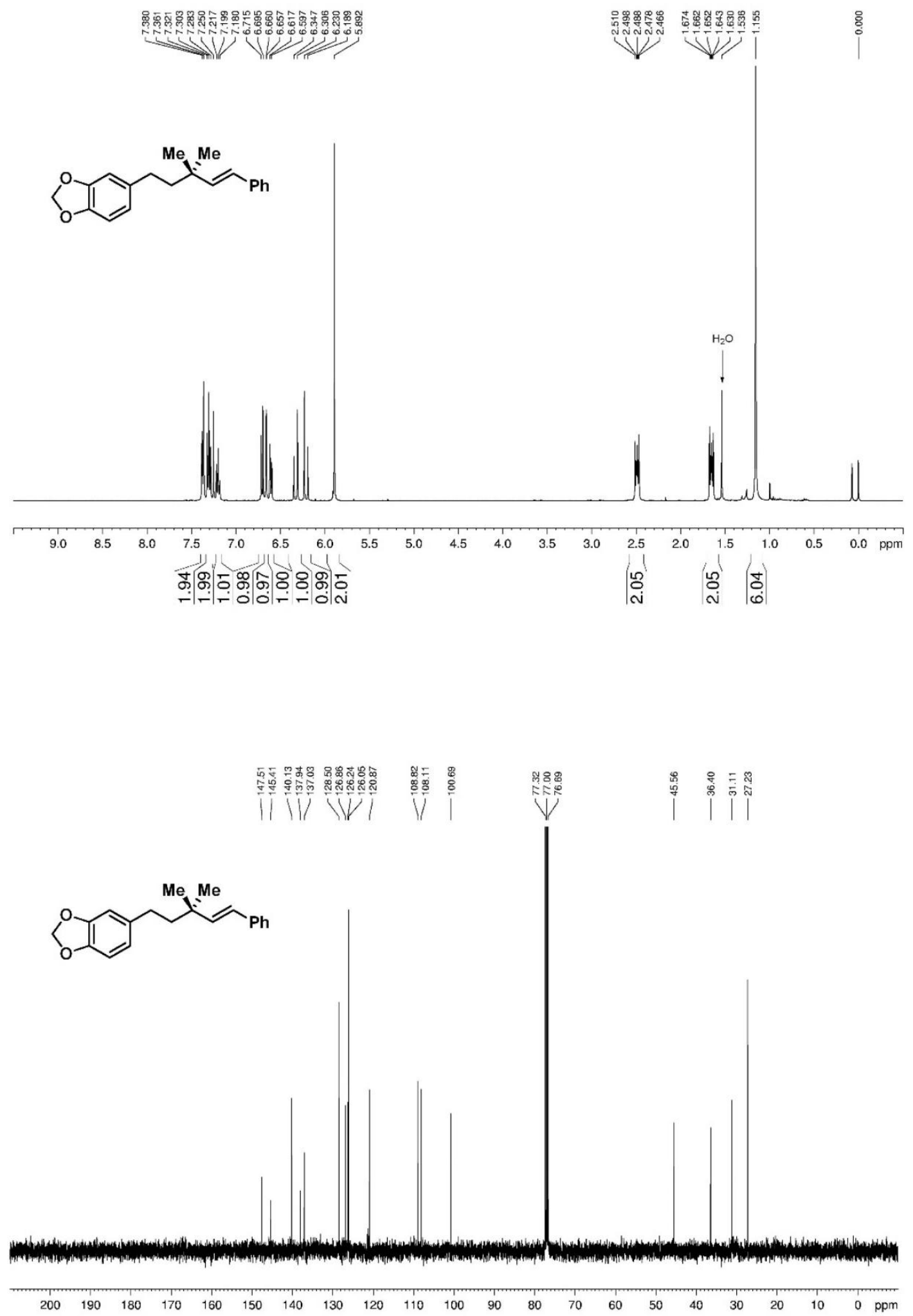
3j; ${ }^{1} \mathrm{H}$ NMR (600 MHz, $\left.\mathrm{CDCl}_{3}\right) ;{ }^{13} \mathrm{C}$ NMR (150 MHz, $\left.\mathrm{CDCl}_{3}\right)$

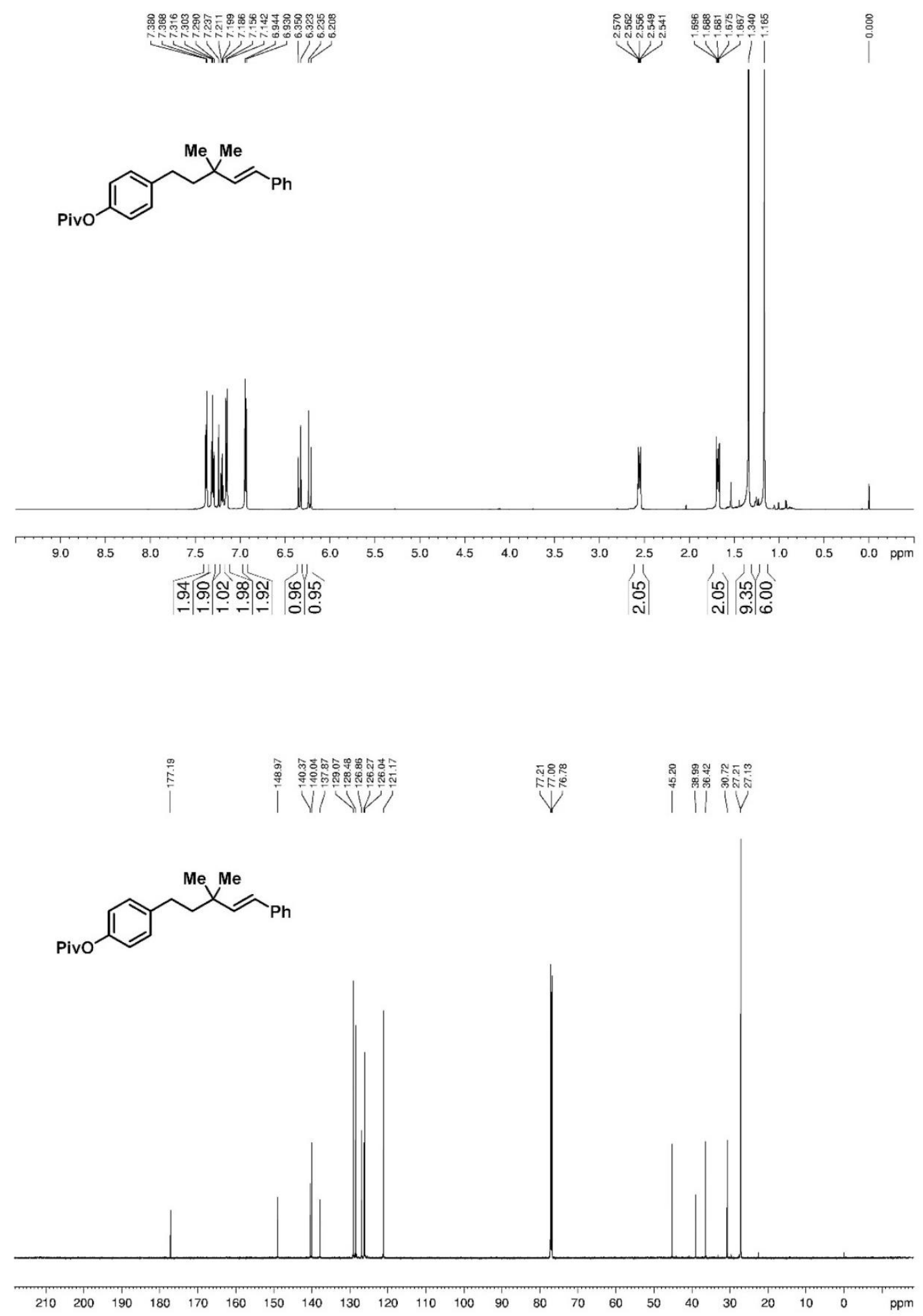


3k; ${ }^{1} \mathrm{H}$ NMR (400 MHz, CDCl3); $\left.{ }^{13} \mathrm{C} \mathrm{NMR} \mathrm{(100} \mathrm{MHz,} \mathrm{CDCl}\right)$

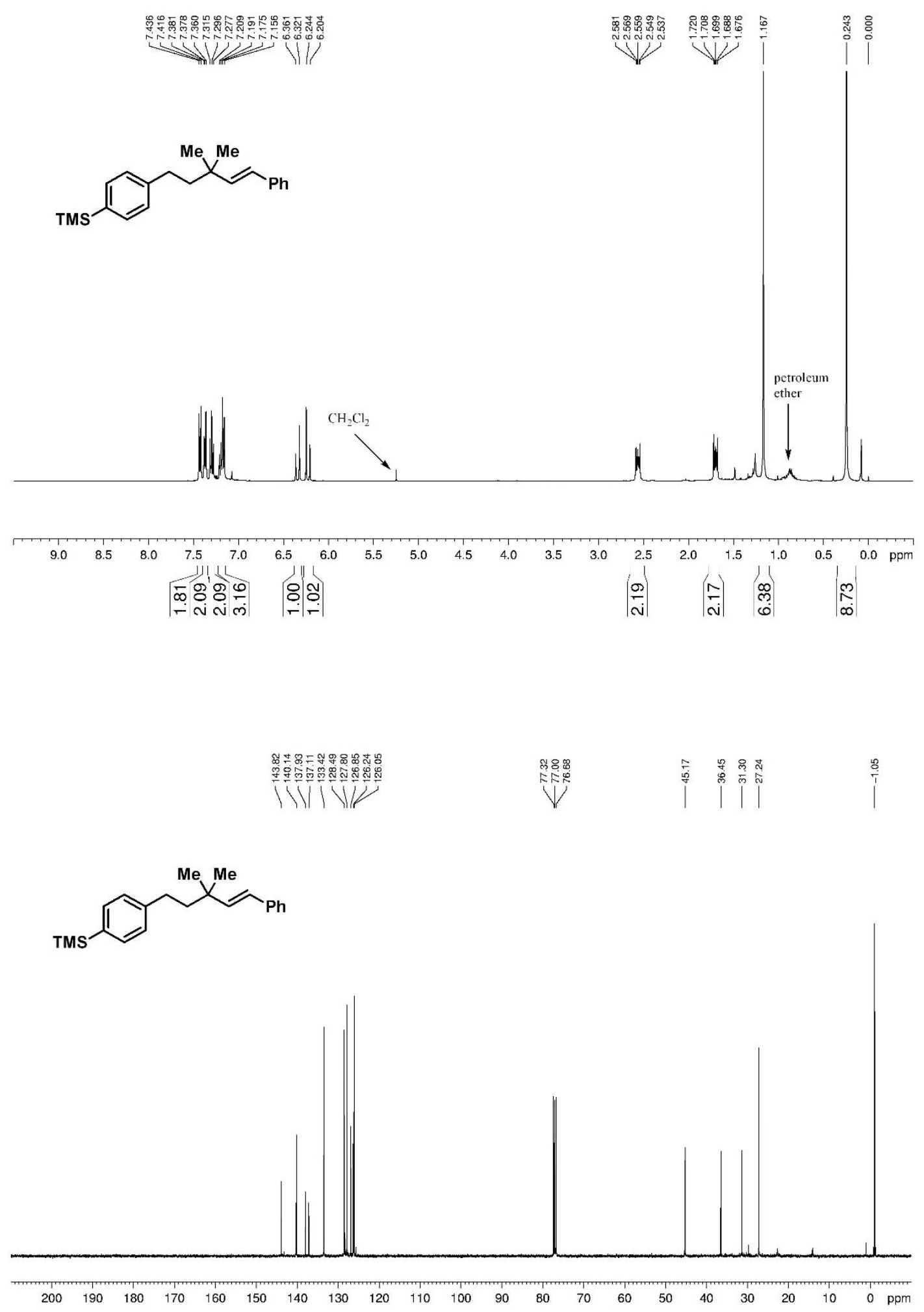


3I; ${ }^{1} \mathrm{H}$ NMR (400 MHz, $\left.\mathrm{CDCl}_{3}\right) ;{ }^{13} \mathrm{C}$ NMR (100 $\left.\mathrm{MHz}, \mathrm{CDCl}_{3}\right)$

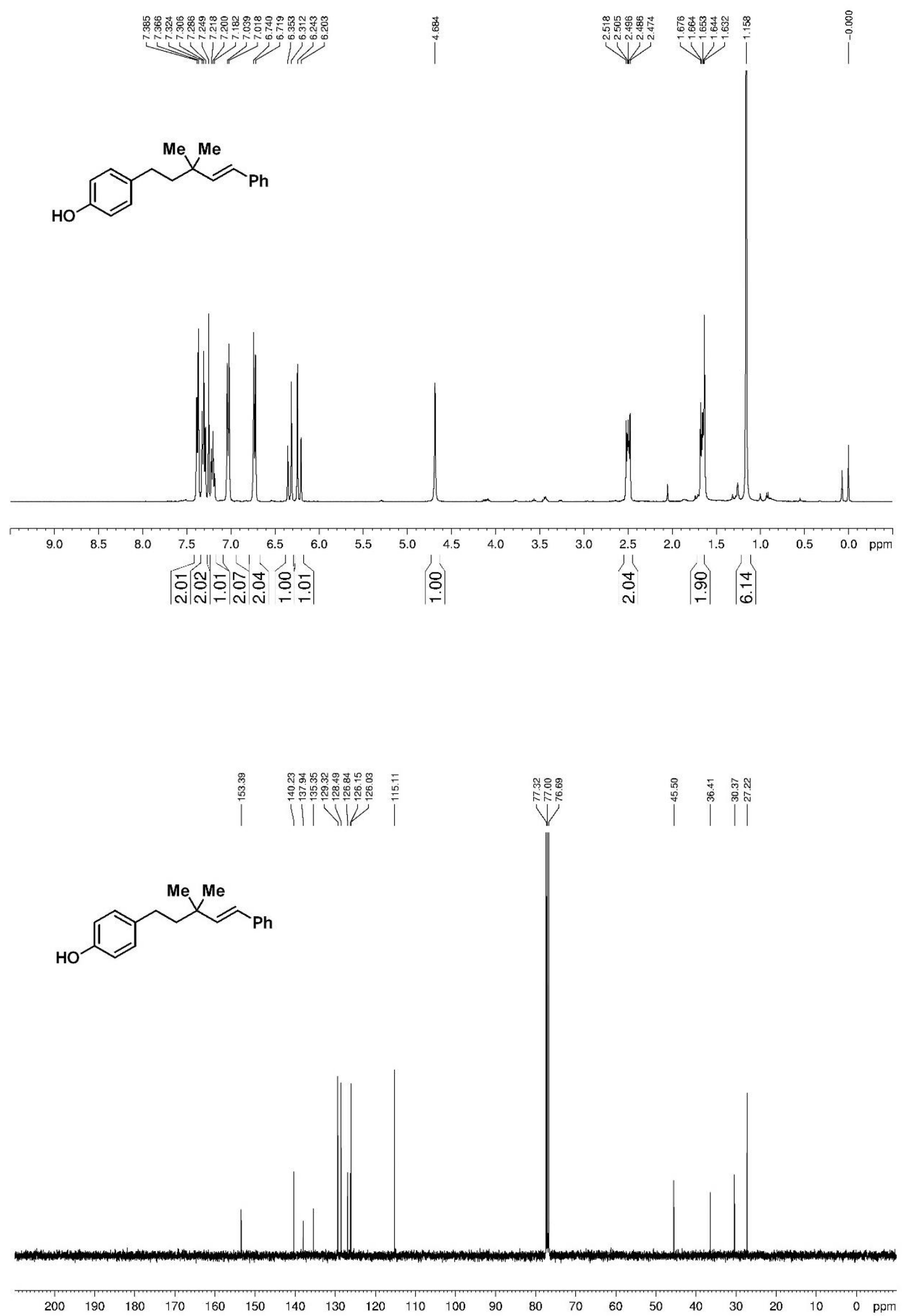


3m; ${ }^{1} \mathrm{H}$ NMR (400 MHz, CDCl $) ;{ }^{13} \mathrm{C}$ NMR (100 MHz, CDCl3)
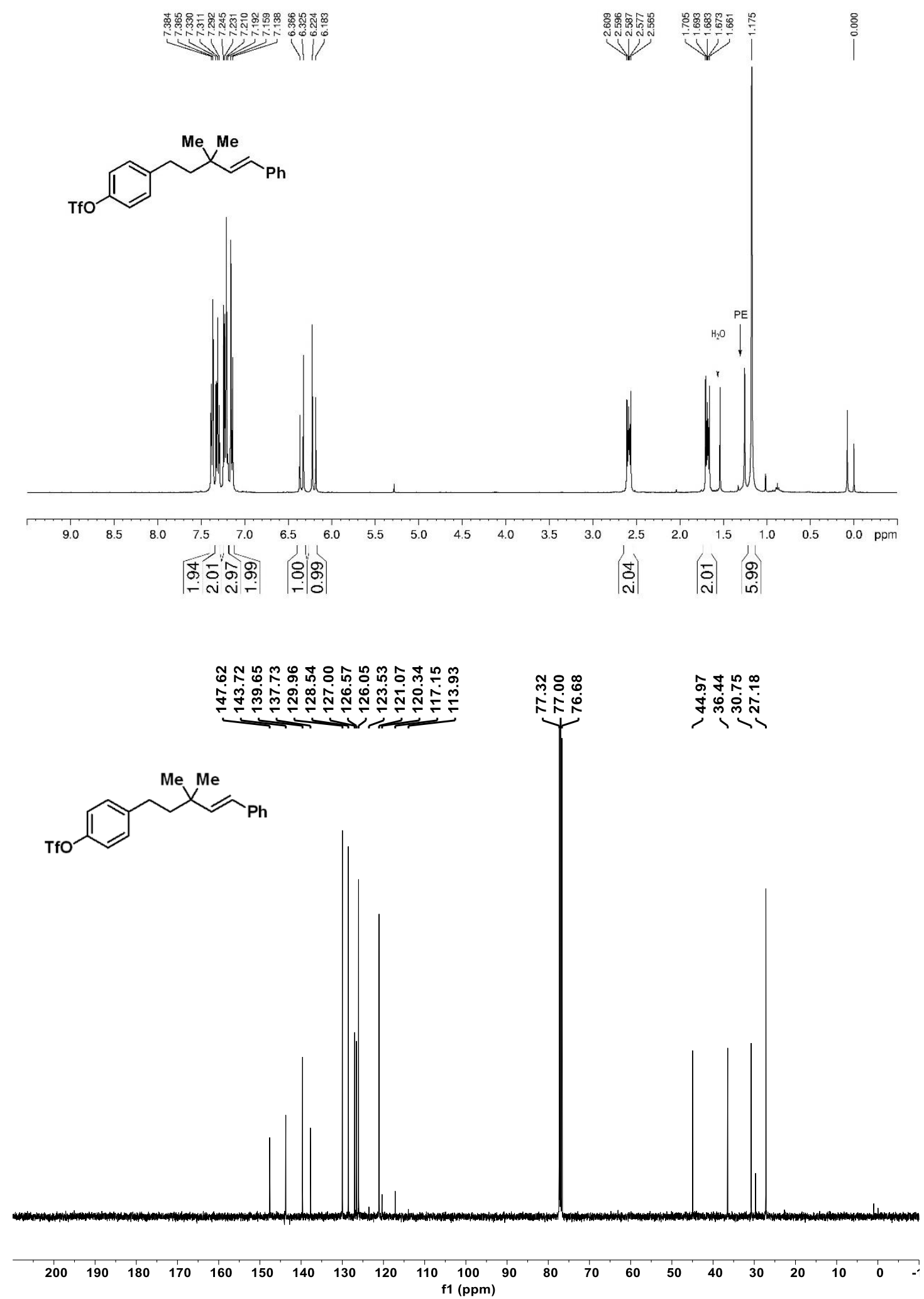
3m; ${ }^{19} \mathrm{~F}$ NMR (376 MHz, CDCl 3 )

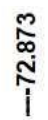<smiles>CC(C)(C=Cc1ccccc1)CCc1ccc(O)cc1</smiles>

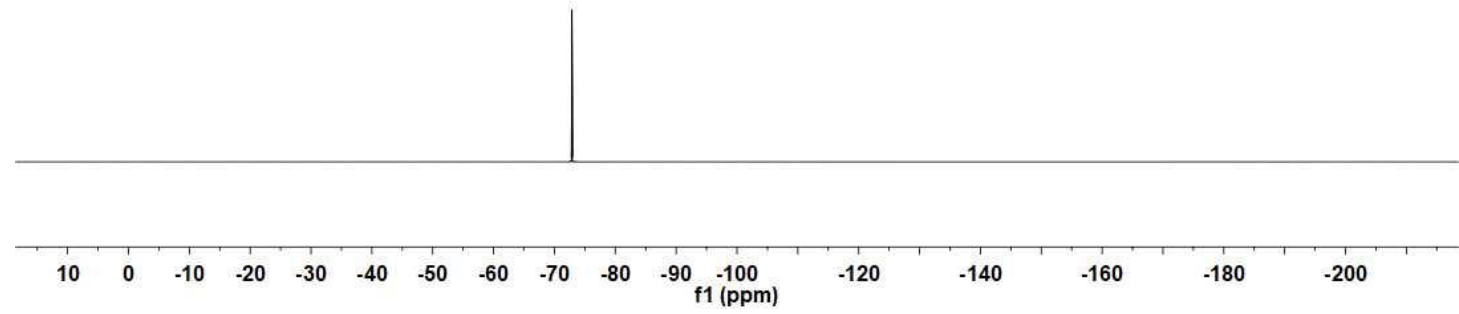


3n; ${ }^{1} \mathrm{H}$ NMR (400 MHz, CDCl$\left.) ;{ }^{13} \mathrm{C} \mathrm{NMR} \mathrm{(100} \mathrm{MHz}, \mathrm{CDCl}_{3}\right)$

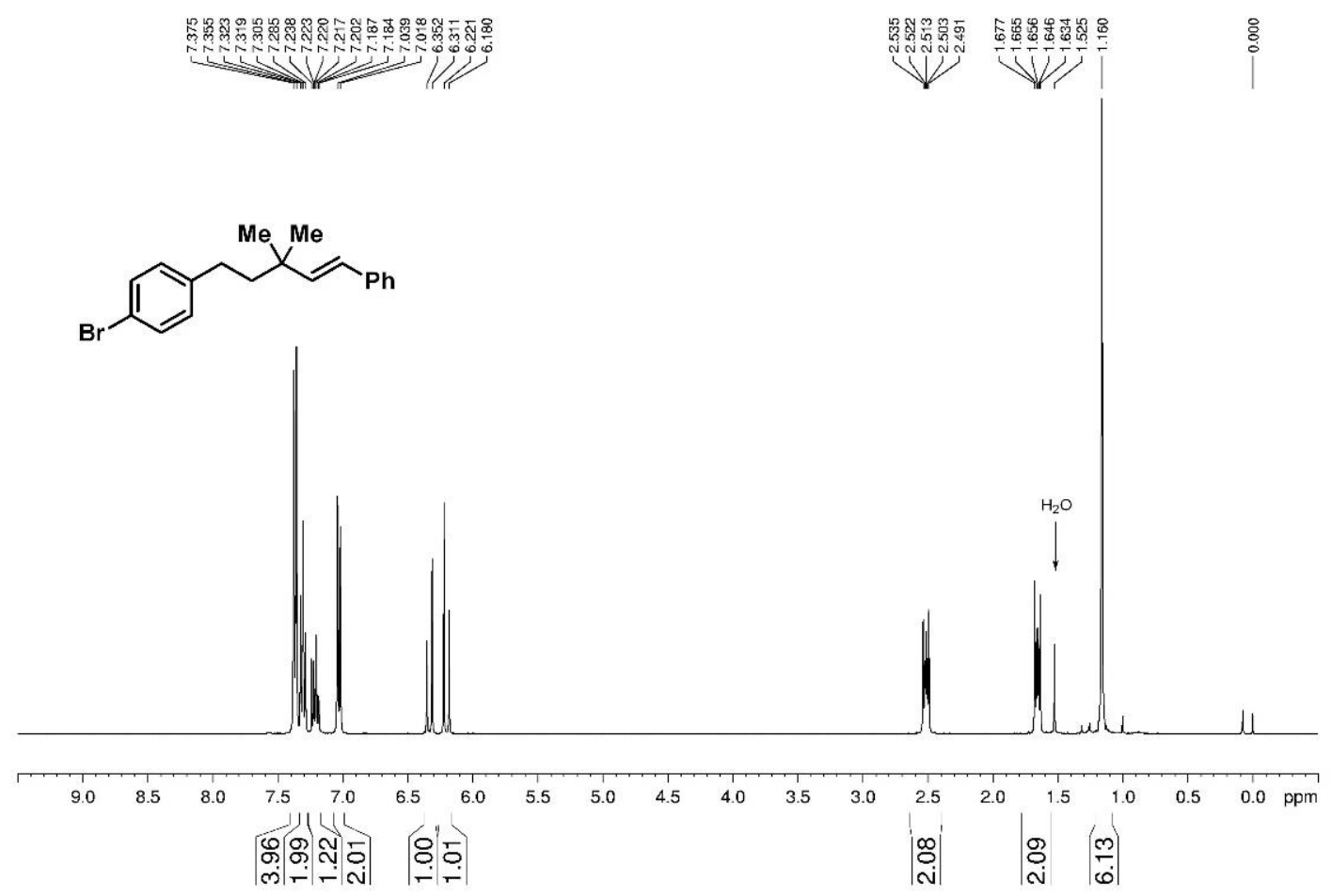

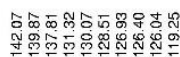

II vivi

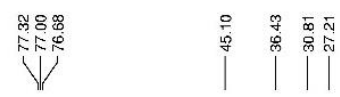

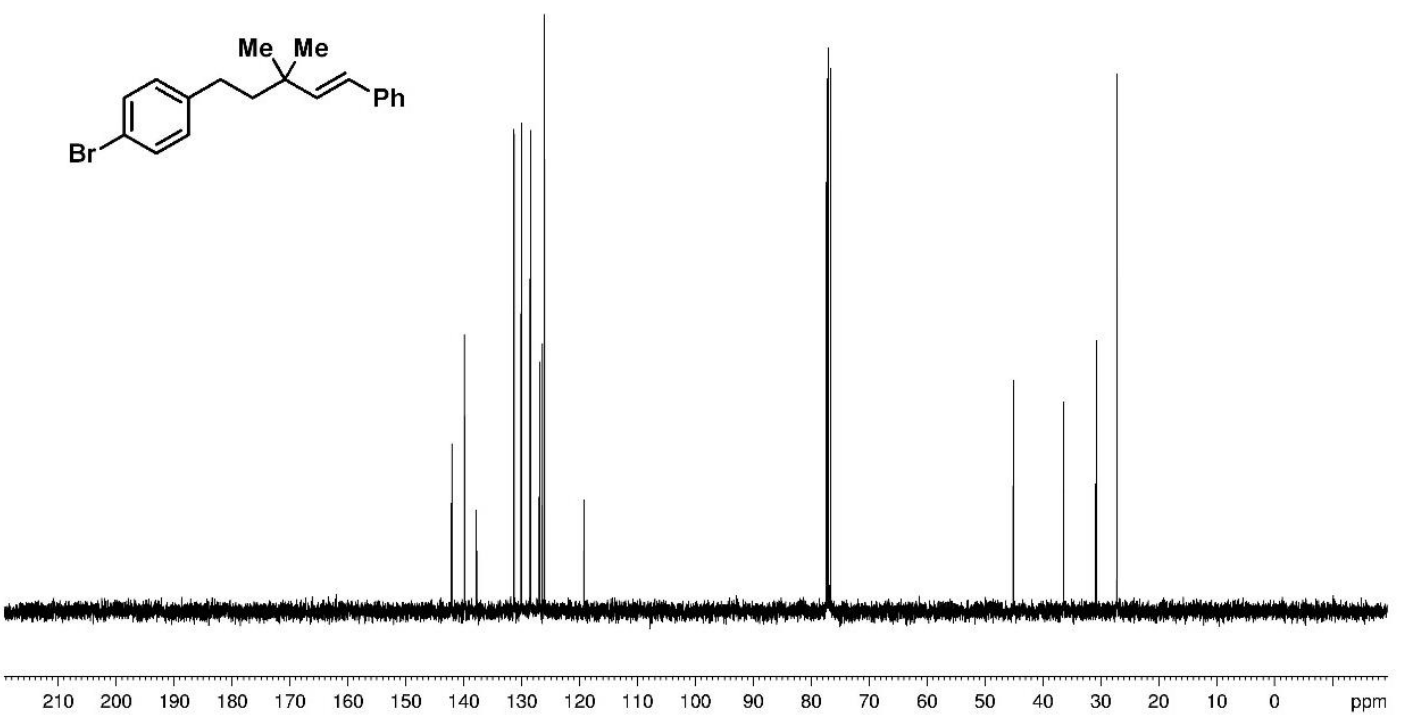




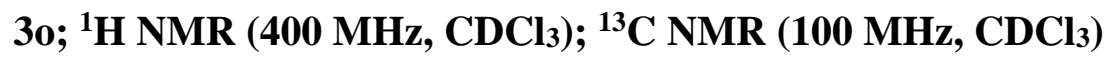
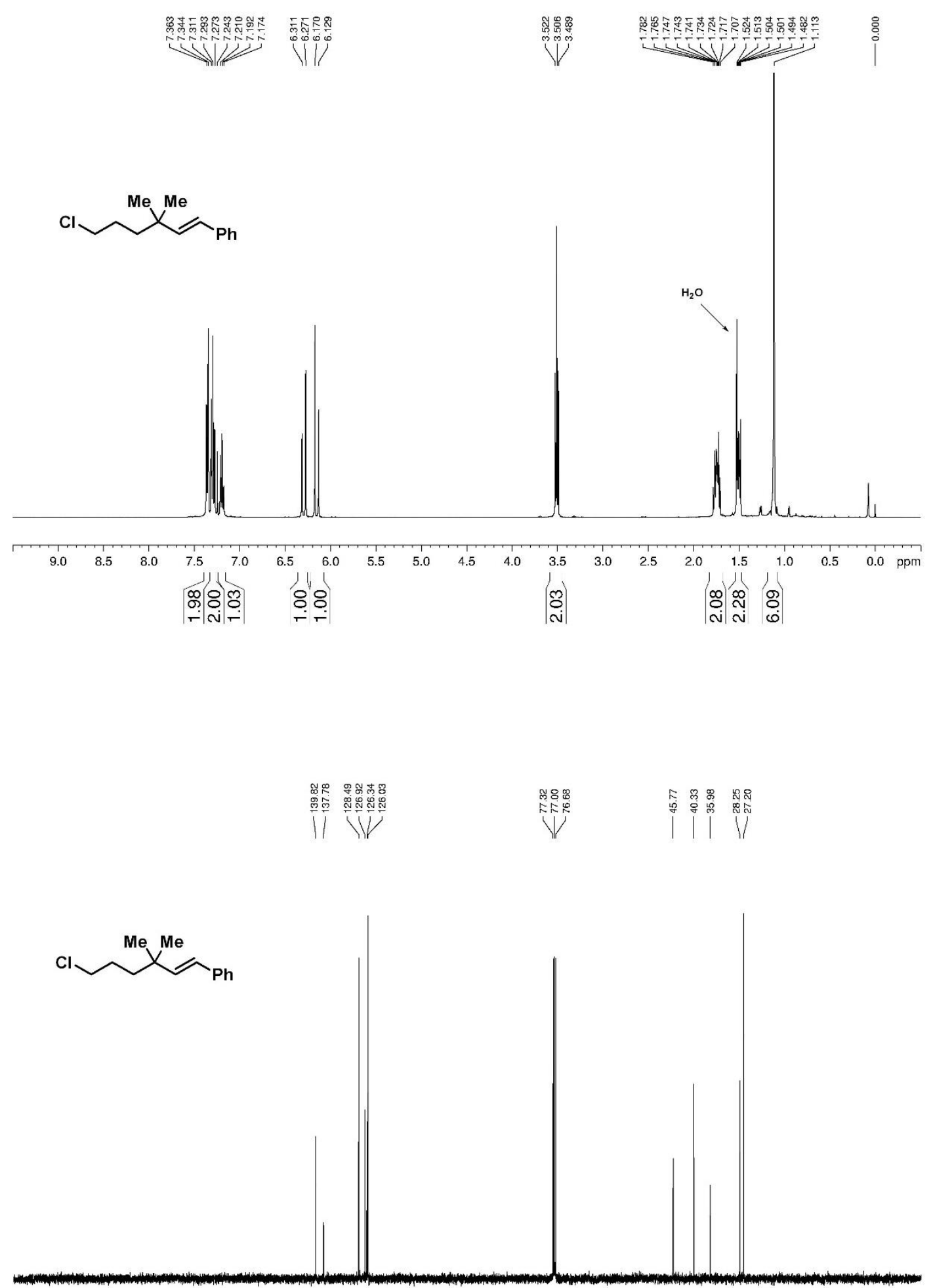

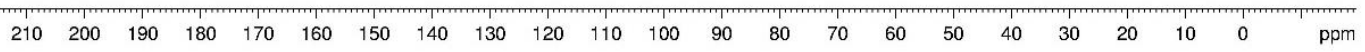


3q; ${ }^{1} \mathrm{H}$ NMR (400 MHz, CDCl$\left.) ;{ }^{13} \mathrm{C} \mathrm{NMR} \mathrm{(100} \mathrm{MHz}, \mathrm{CDCl}_{3}\right)$
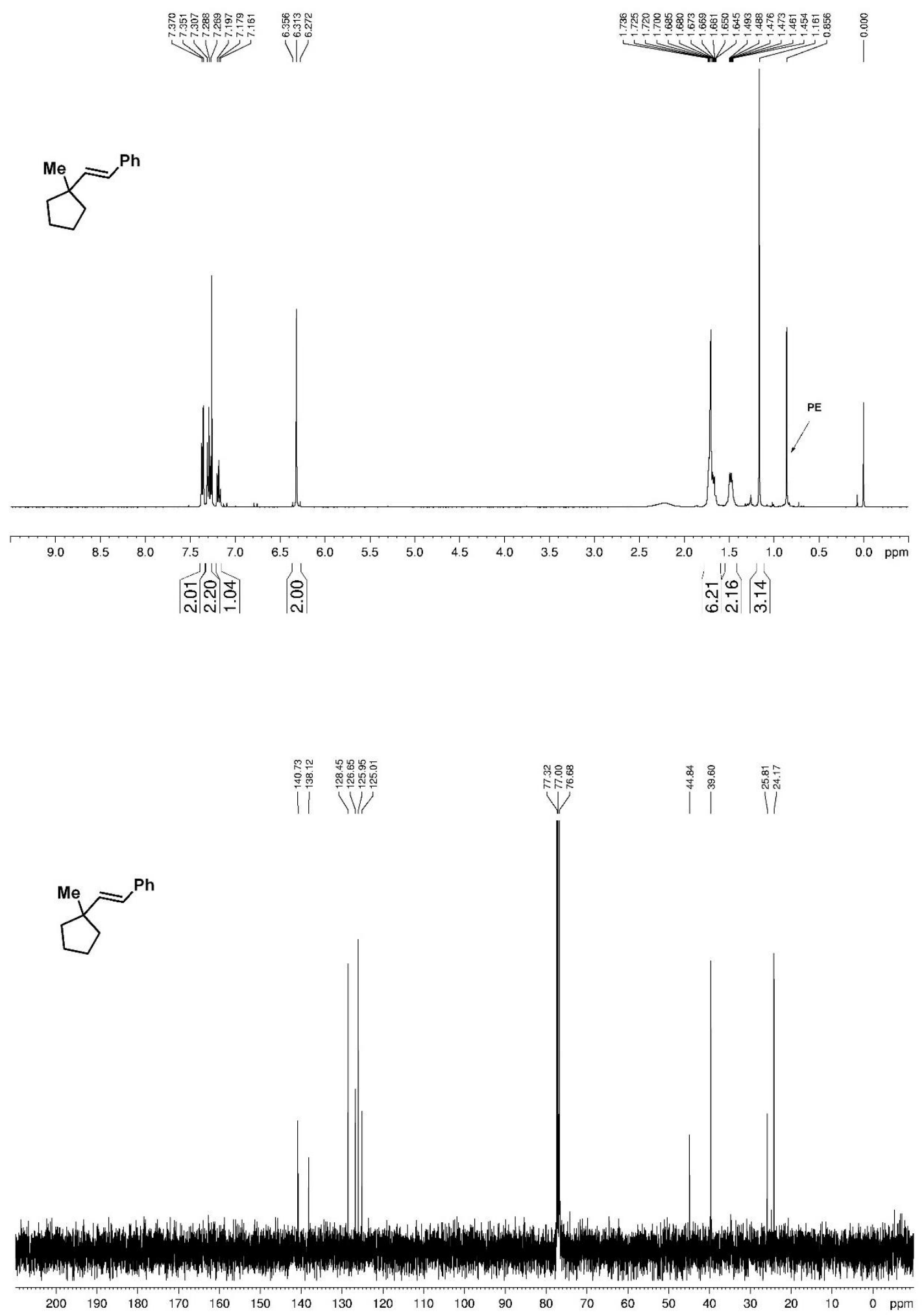
3r; ${ }^{1} \mathrm{H}$ NMR (600 MHz, CDCl 3$\left.) ;{ }^{13} \mathrm{C} \mathrm{NMR} \mathrm{(150} \mathrm{MHz,} \mathrm{CDCl} 3\right)$

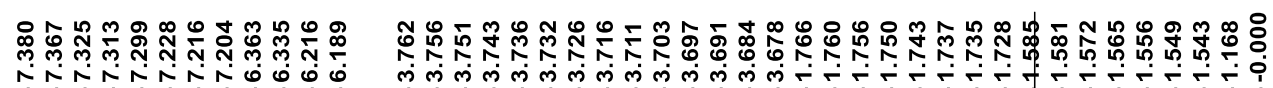
个iN
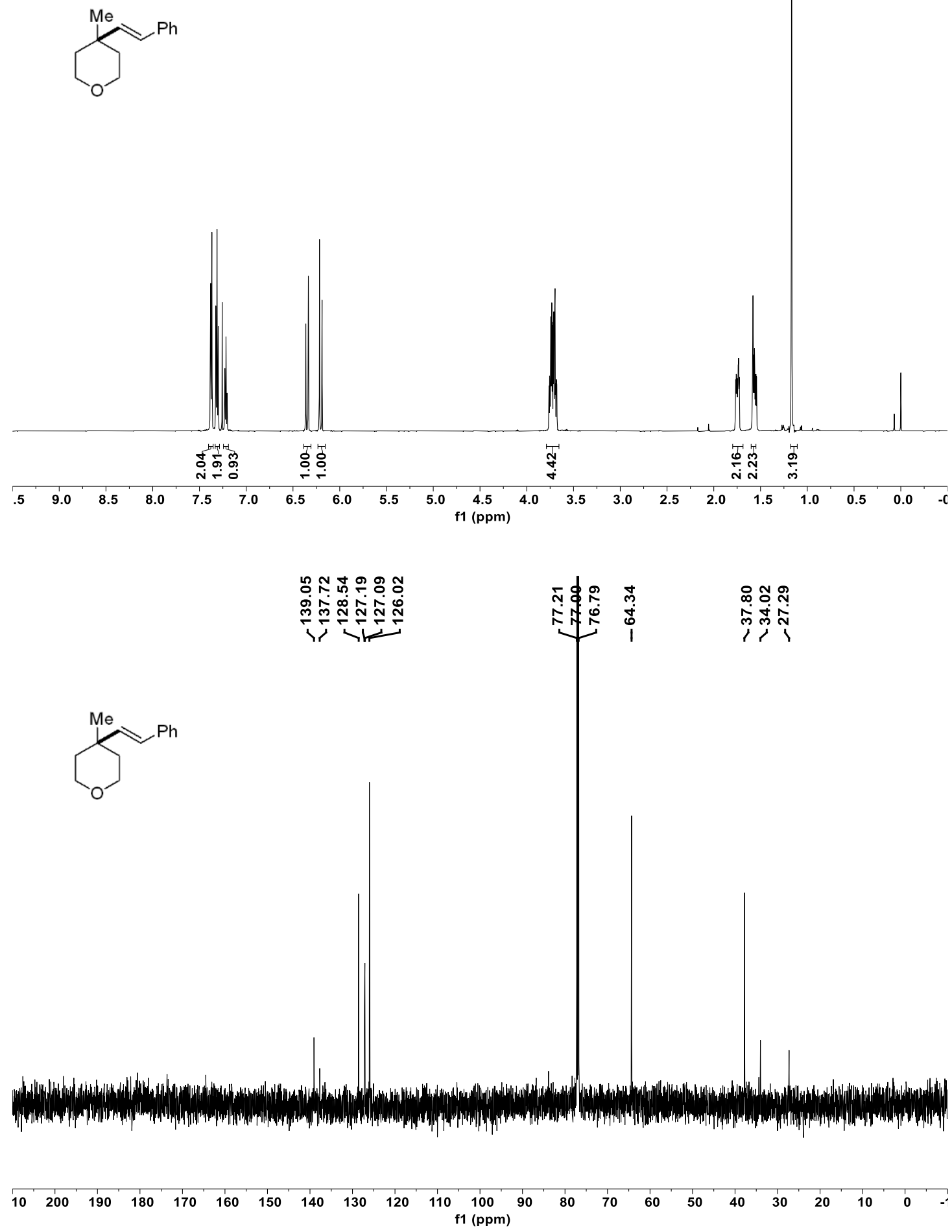
3s; ${ }^{1} \mathrm{H}$ NMR (400 MHz, $\left.\mathrm{CDCl}_{3}\right) ;{ }^{13} \mathrm{C}$ NMR (100 MHz, $\left.\mathrm{CDCl}_{3}\right)$
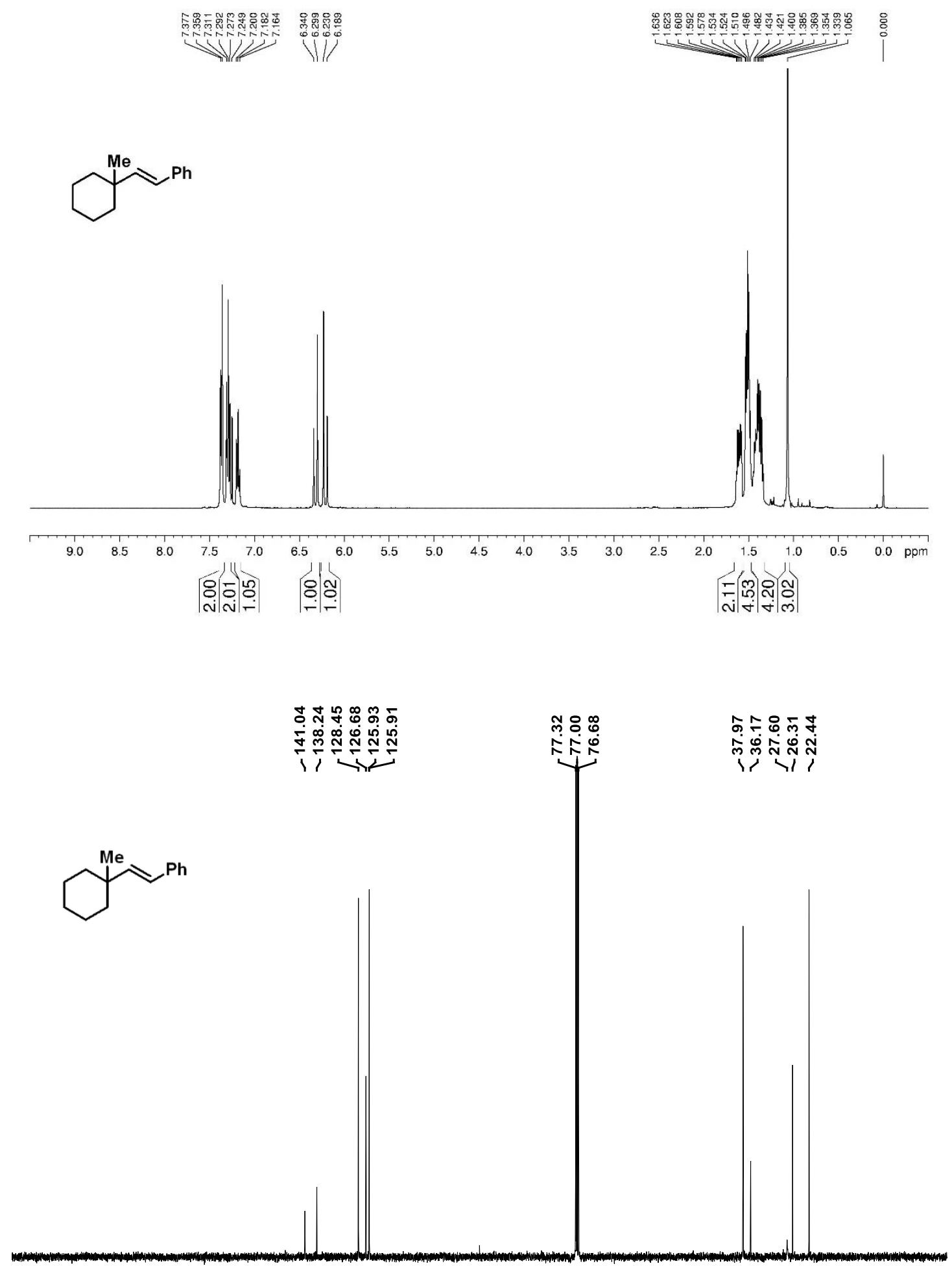

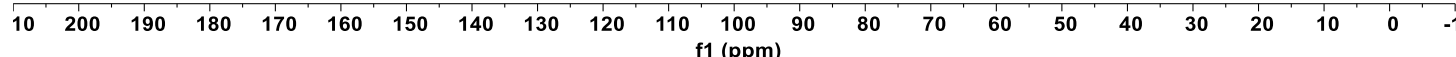


3t; ${ }^{1} \mathrm{H}$ NMR (400 MHz, CDCl $) ;{ }^{13} \mathrm{C}$ NMR (100 MHz, CDCl $)$
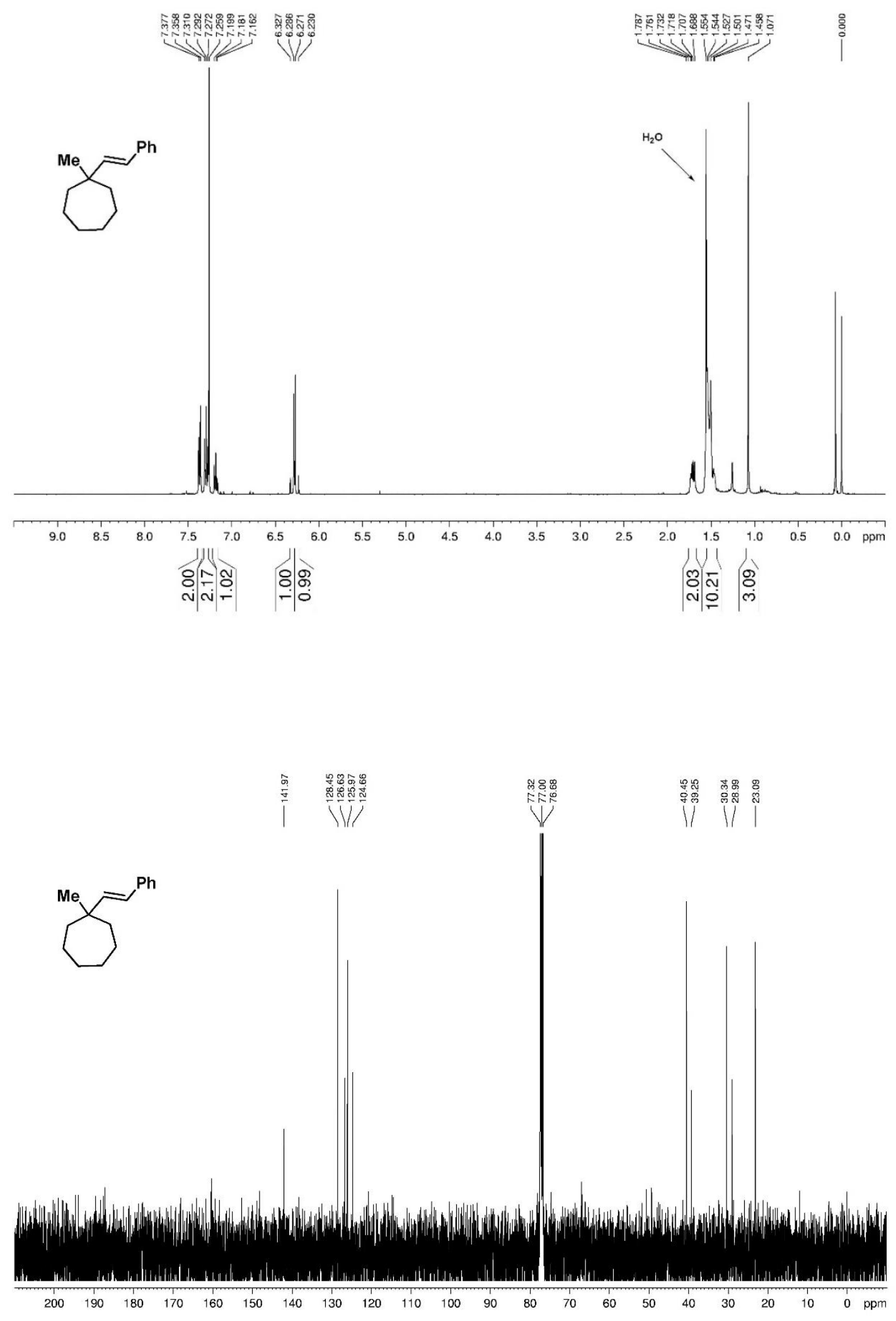
3u; ${ }^{1} \mathrm{H}$ NMR (400 MHz, CDCl $)$; ${ }^{13} \mathrm{C}$ NMR (100 MHz, CDCl $)$
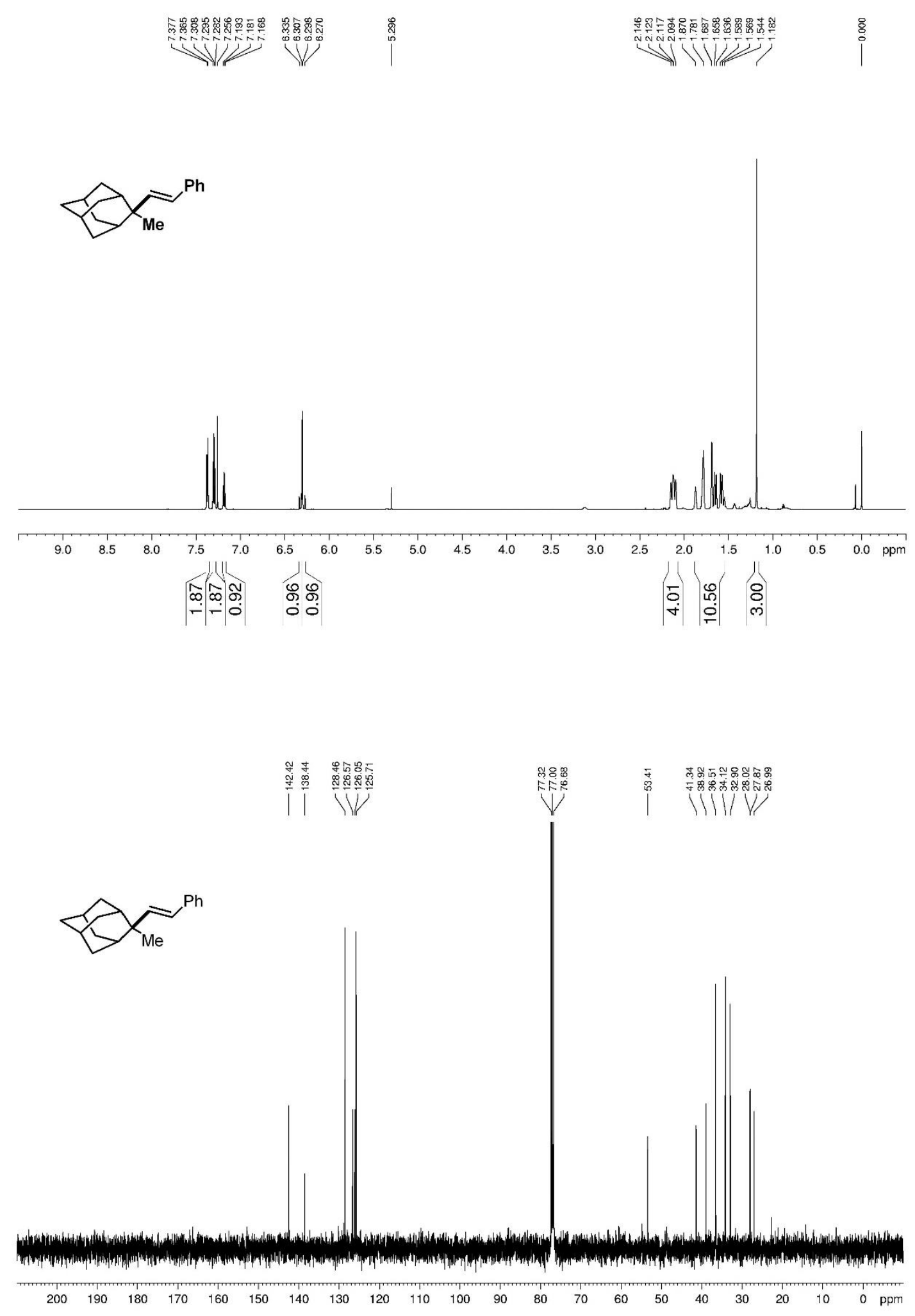
3v; ${ }^{1} \mathrm{H}$ NMR (400 MHz, $\left.\mathrm{CDCl}_{3}\right) ;{ }^{13} \mathrm{C}$ NMR (100 MHz, $\left.\mathrm{CDCl}_{3}\right)$
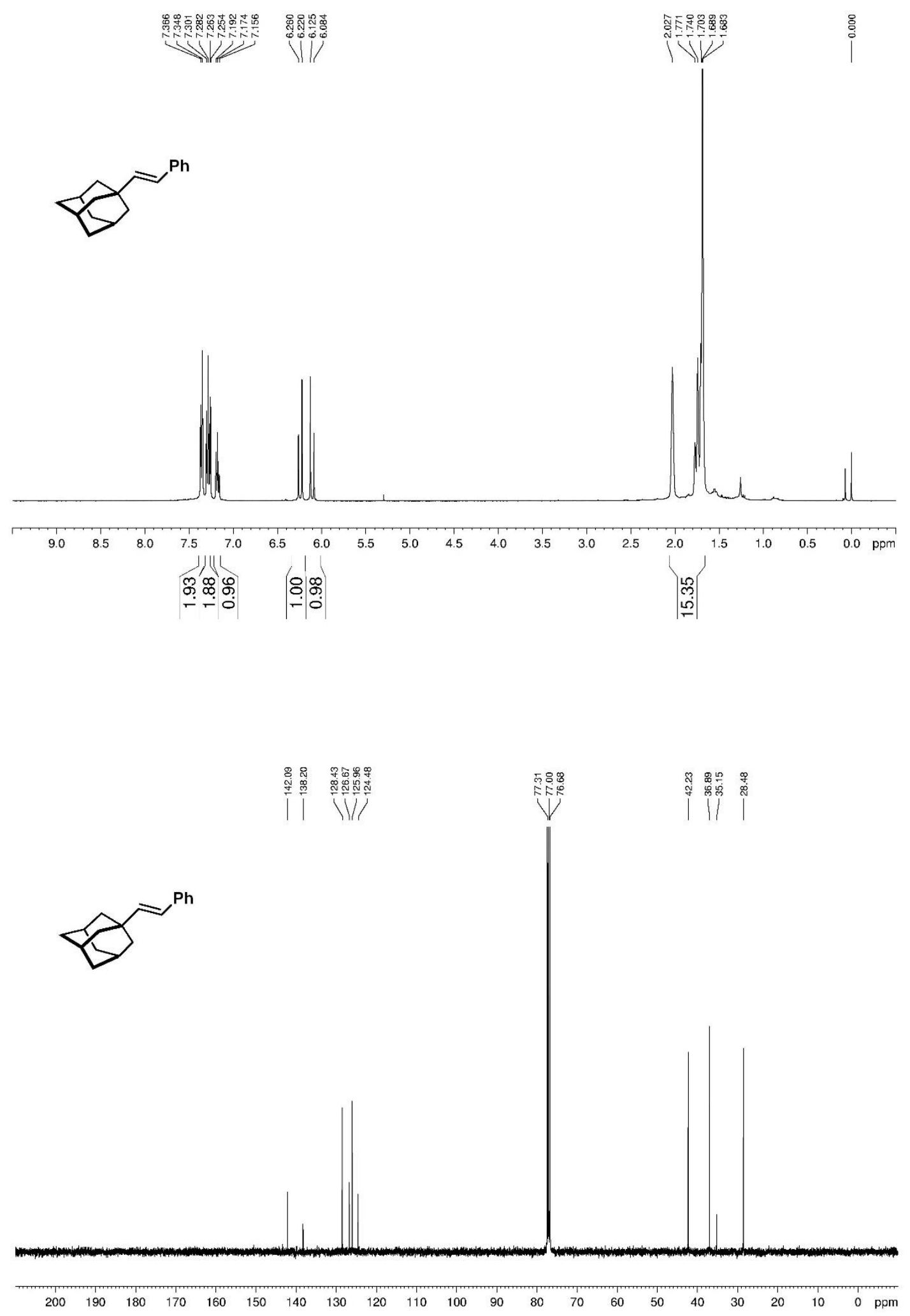
3w; ${ }^{1} \mathrm{H}$ NMR (400 $\left.\mathrm{MHz}, \mathrm{CDCl}_{3}\right) ;{ }^{13} \mathrm{C} \mathrm{NMR}\left(100 \mathrm{MHz}, \mathrm{CDCl}_{3}\right)$
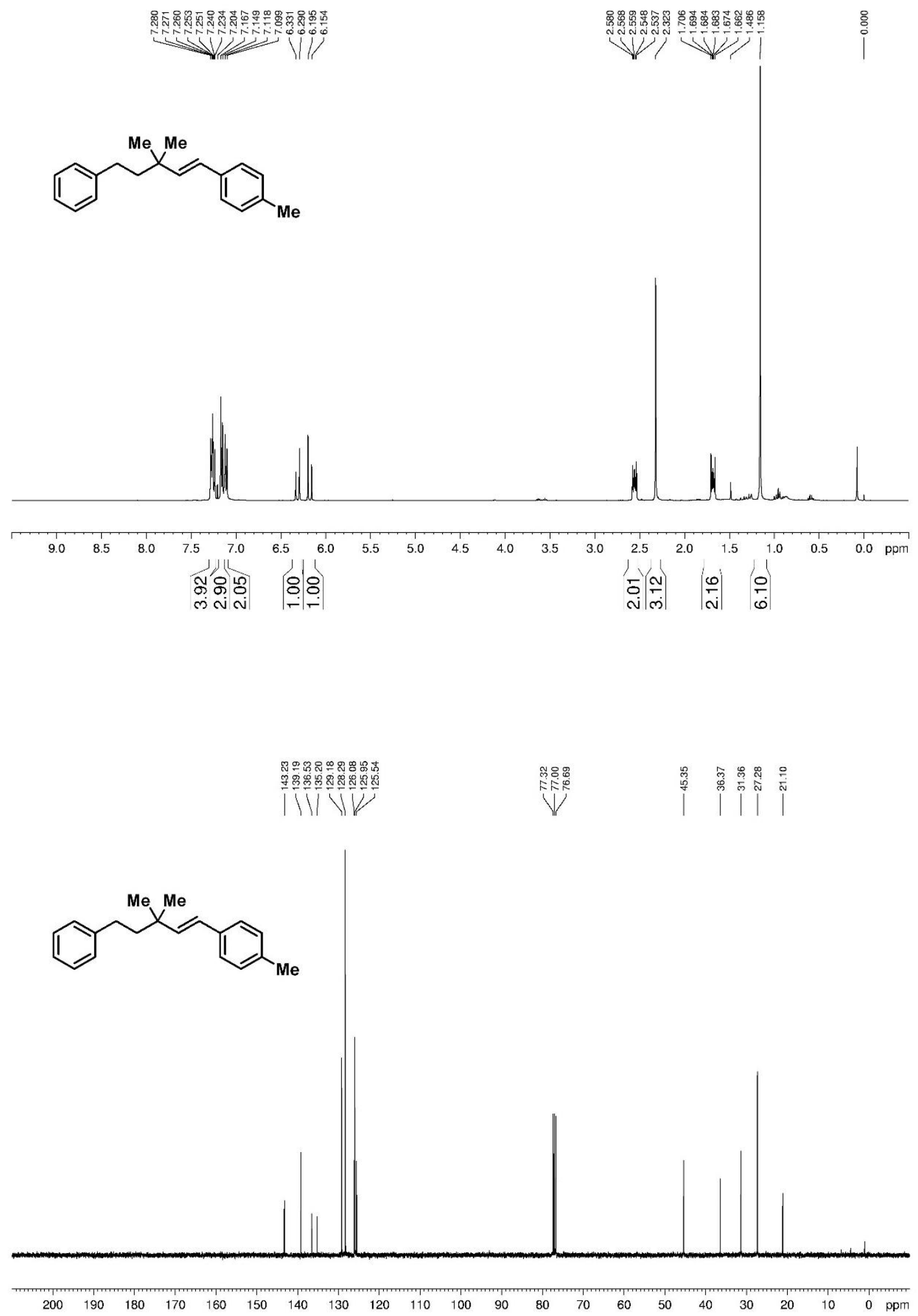

S98 
3x; ${ }^{1} \mathrm{H}$ NMR (400 MHz, CDCl 3$) ;{ }^{13} \mathrm{C}$ NMR (100 MHz, CDCl $)$
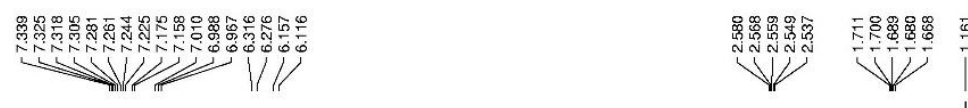

$\stackrel{\circ}{i}$
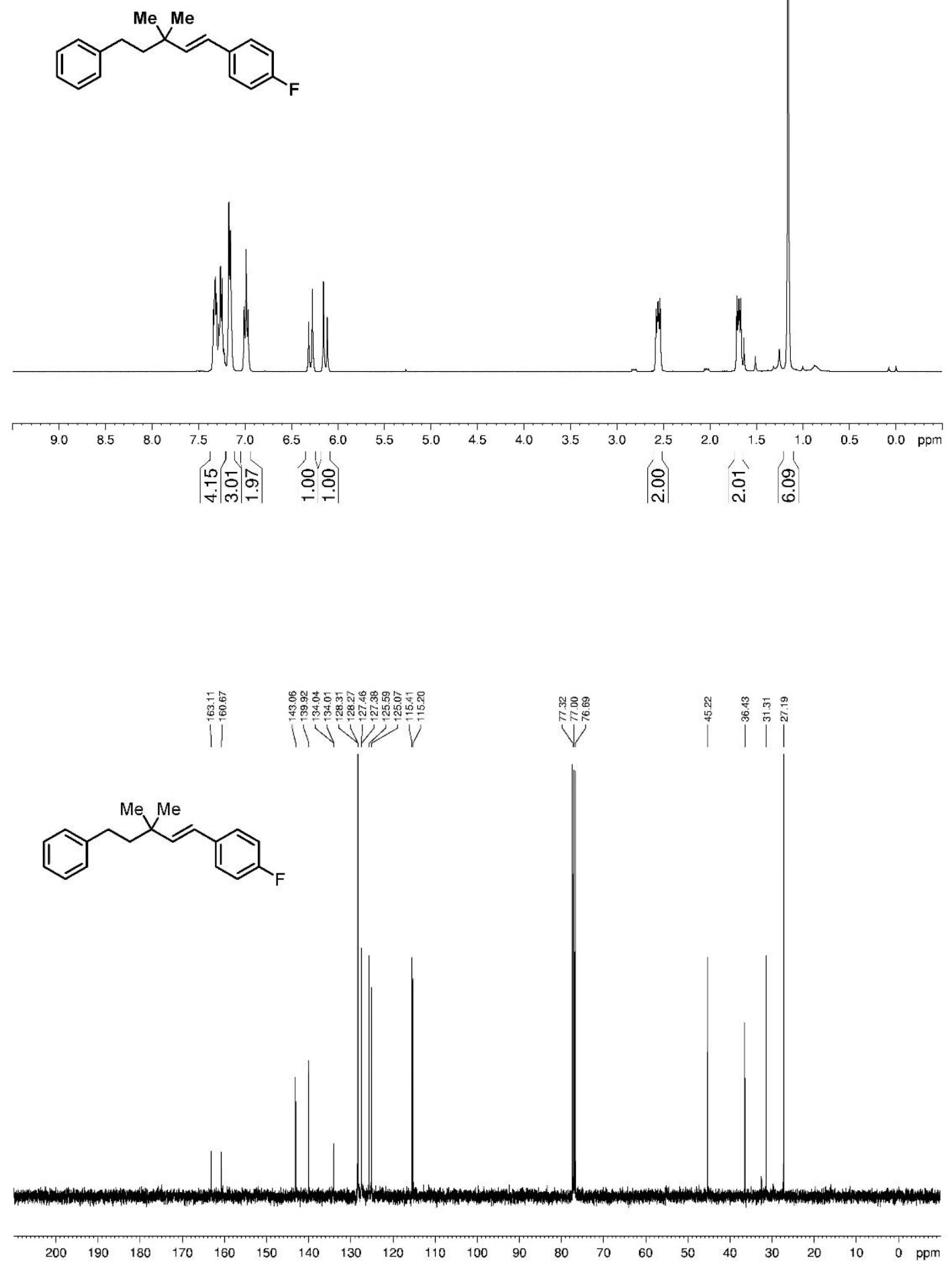

S99 
3x; ${ }^{19}$ F NMR (376 MHz, CDCl 3 )

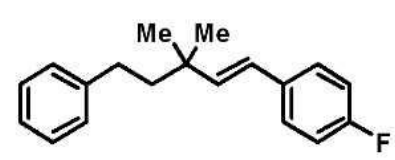

$\stackrel{\substack{0 \\ \stackrel{6}{i}}}{i}$

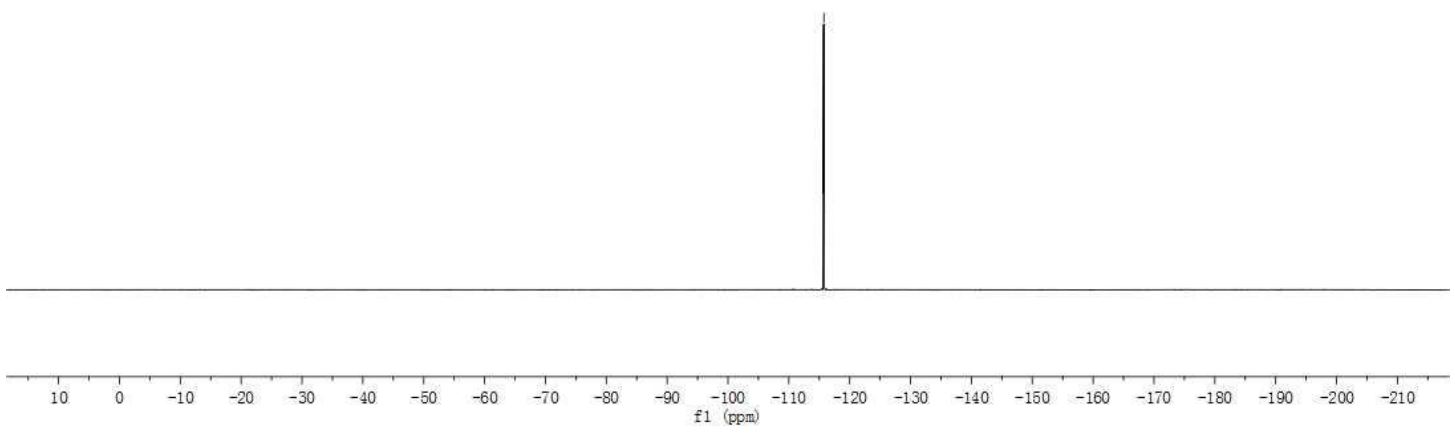

S100 


\section{3y; ${ }^{1} \mathrm{H}$ NMR (600 MHz, $\left.\mathrm{CDCl}_{3}\right) ;{ }^{13} \mathrm{C}$ NMR $\left(150 \mathrm{MHz}, \mathrm{CDCl}_{3}\right)$}
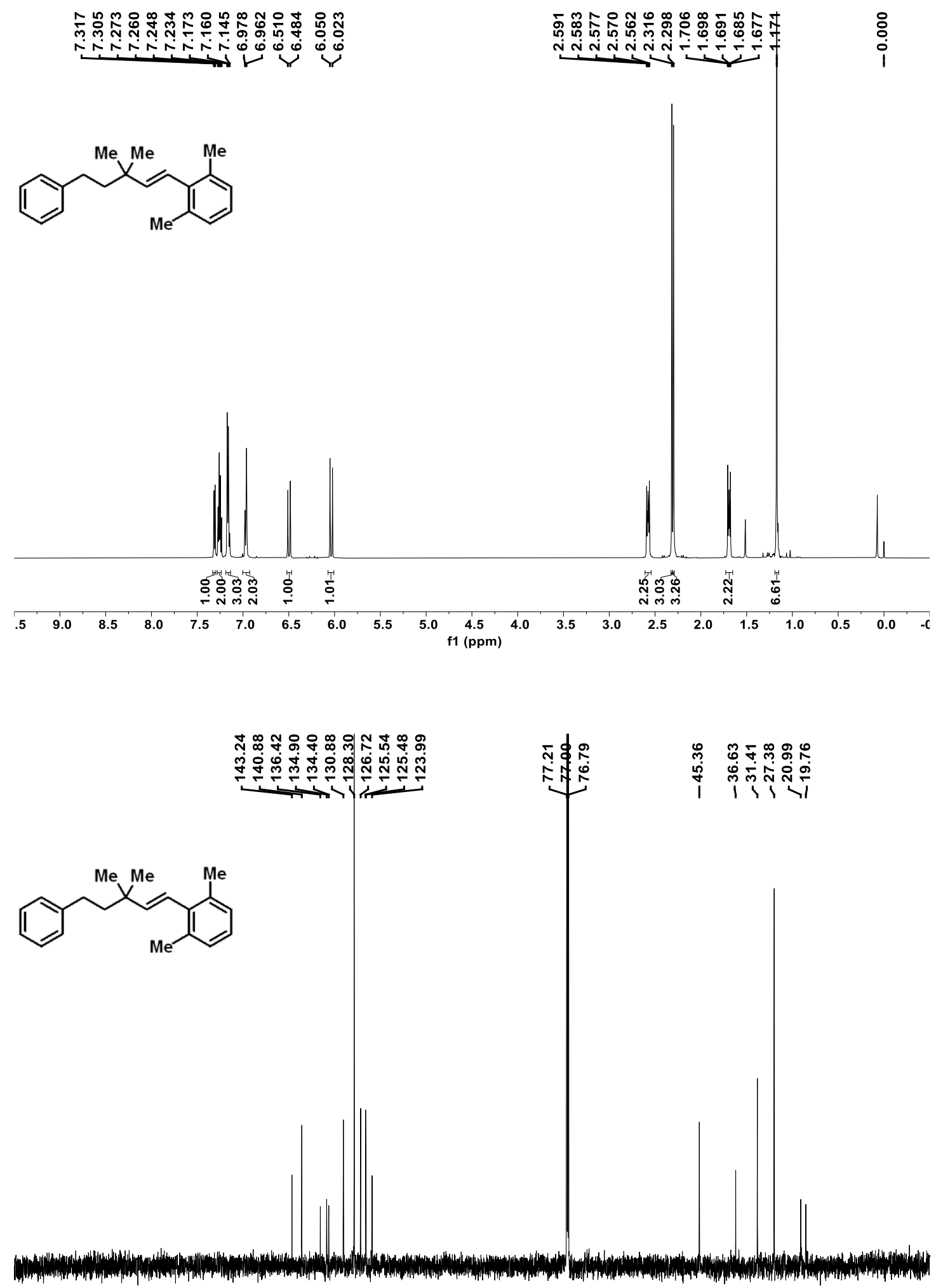

\begin{tabular}{|llllllllllllllllllllll}
10 & 200 & 190 & 180 & 170 & 160 & 150 & 140 & 130 & 120 & $110 \begin{array}{c}100 \\
\mathrm{f} 1(\mathrm{ppm})\end{array}$ & 90 & 80 & 70 & 60 & 50 & 40 & 30 & 20 & 10 & 0 & -
\end{tabular} 
3z; ${ }^{1} \mathrm{H}$ NMR (400 MHz, $\left.\mathrm{CDCl}_{3}\right) ;{ }^{13} \mathrm{C}$ NMR (100 MHz, $\left.\mathrm{CDCl}_{3}\right)$

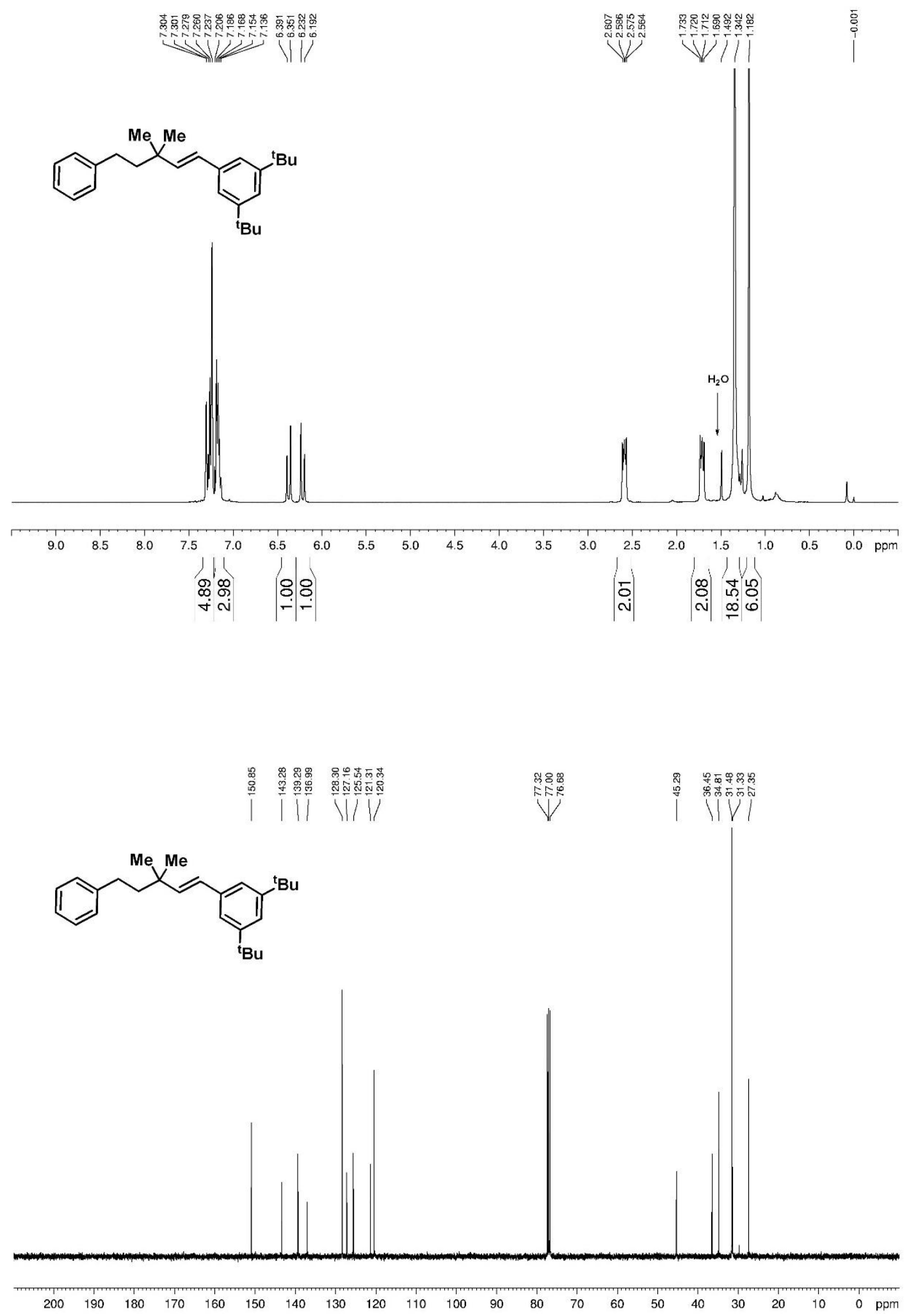


3aa; ${ }^{1} \mathrm{H}$ NMR (400 MHz, $\left.\mathrm{CDCl}_{3}\right) ;{ }^{13} \mathrm{C}$ NMR (100 MHz, $\left.\mathrm{CDCl}_{3}\right)$

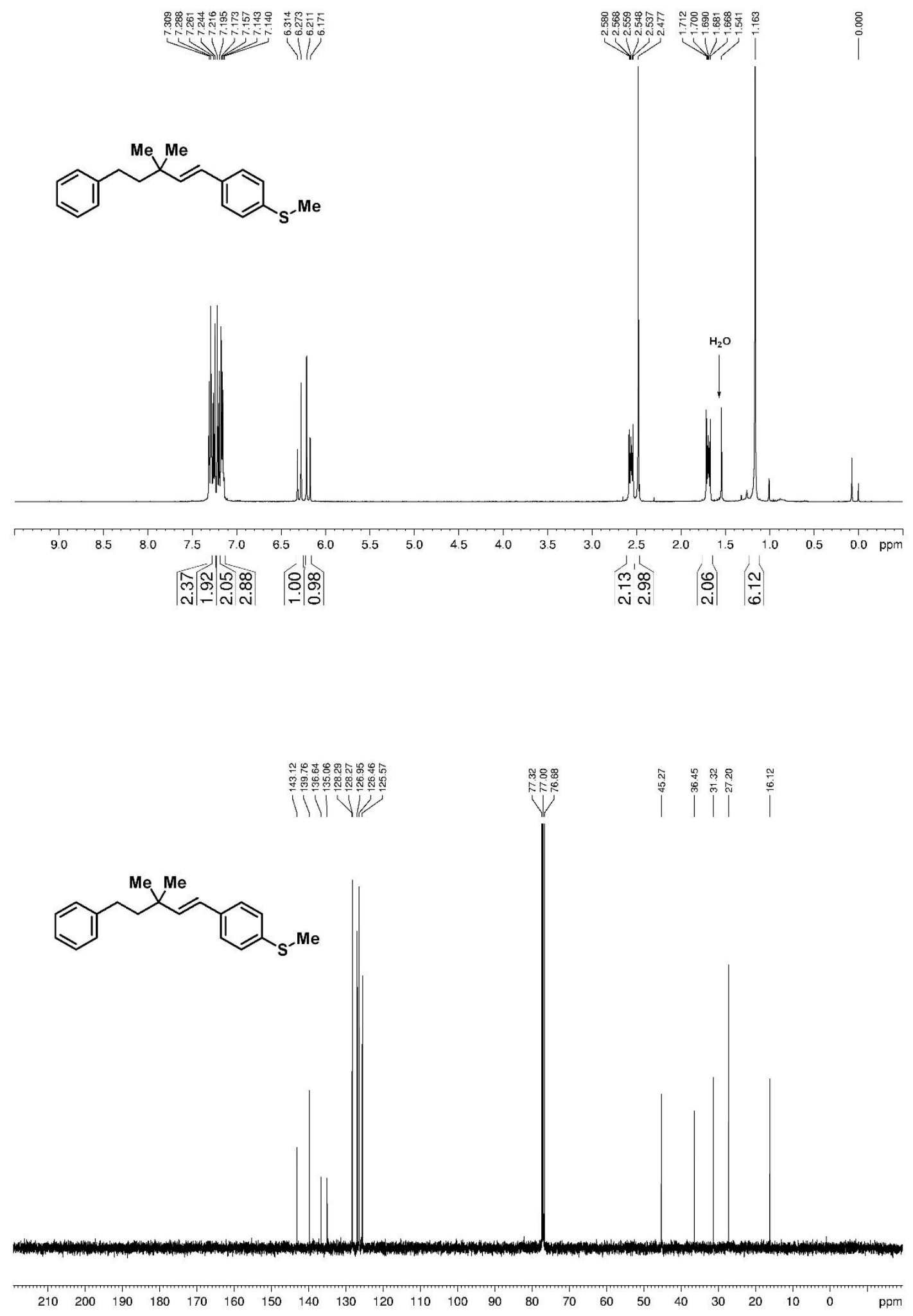


3ab; ${ }^{1} \mathrm{H}$ NMR (400 MHz, $\left.\mathrm{CDCl}_{3}\right) ;{ }^{13} \mathrm{C}$ NMR (100 MHz, $\left.\mathrm{CDCl}_{3}\right)$

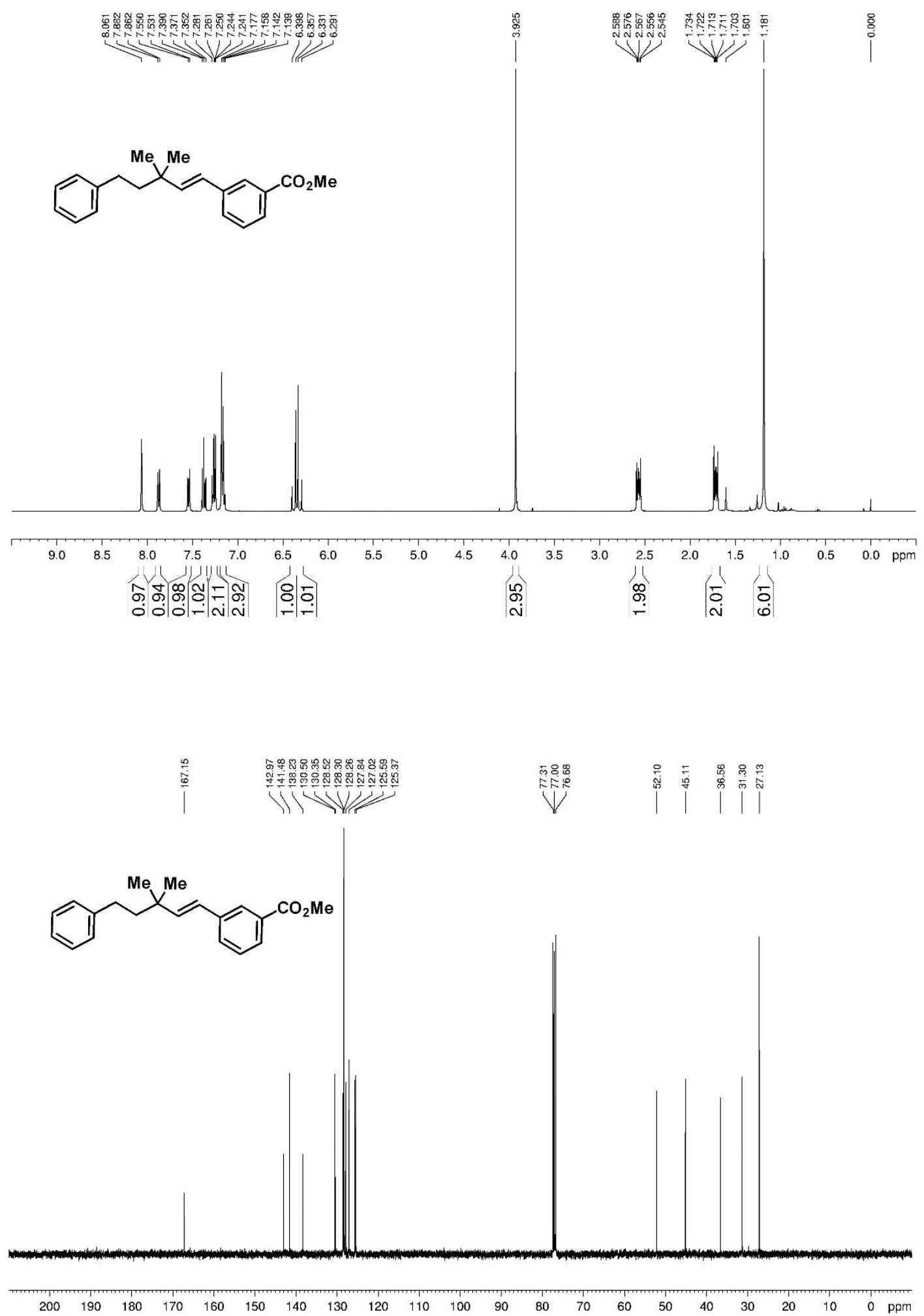


3ac; ${ }^{1} \mathrm{H}$ NMR (400 MHz, $\left.\mathrm{CDCl}_{3}\right) ;{ }^{13} \mathrm{C}$ NMR (100 $\left.\mathrm{MHz}, \mathrm{CDCl}_{3}\right)$

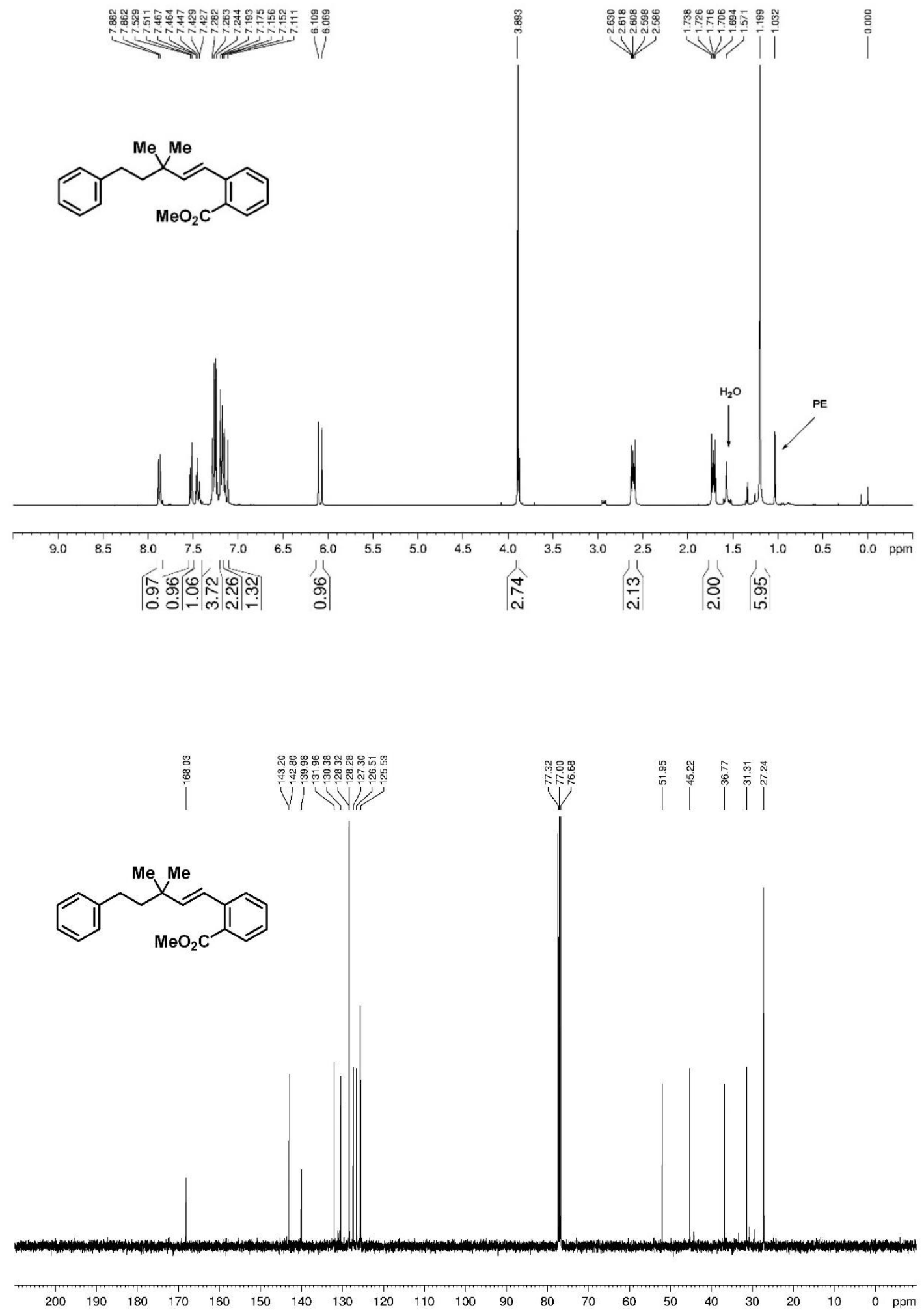


3ad; ${ }^{1} \mathrm{H}$ NMR (400 MHz, $\left.\mathrm{CDCl}_{3}\right) ;{ }^{13} \mathrm{C}$ NMR (100 MHz, $\left.\mathrm{CDCl}_{3}\right)$
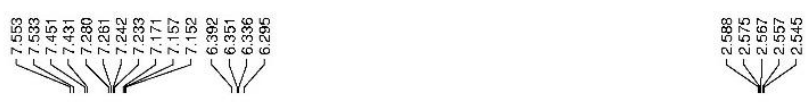

I
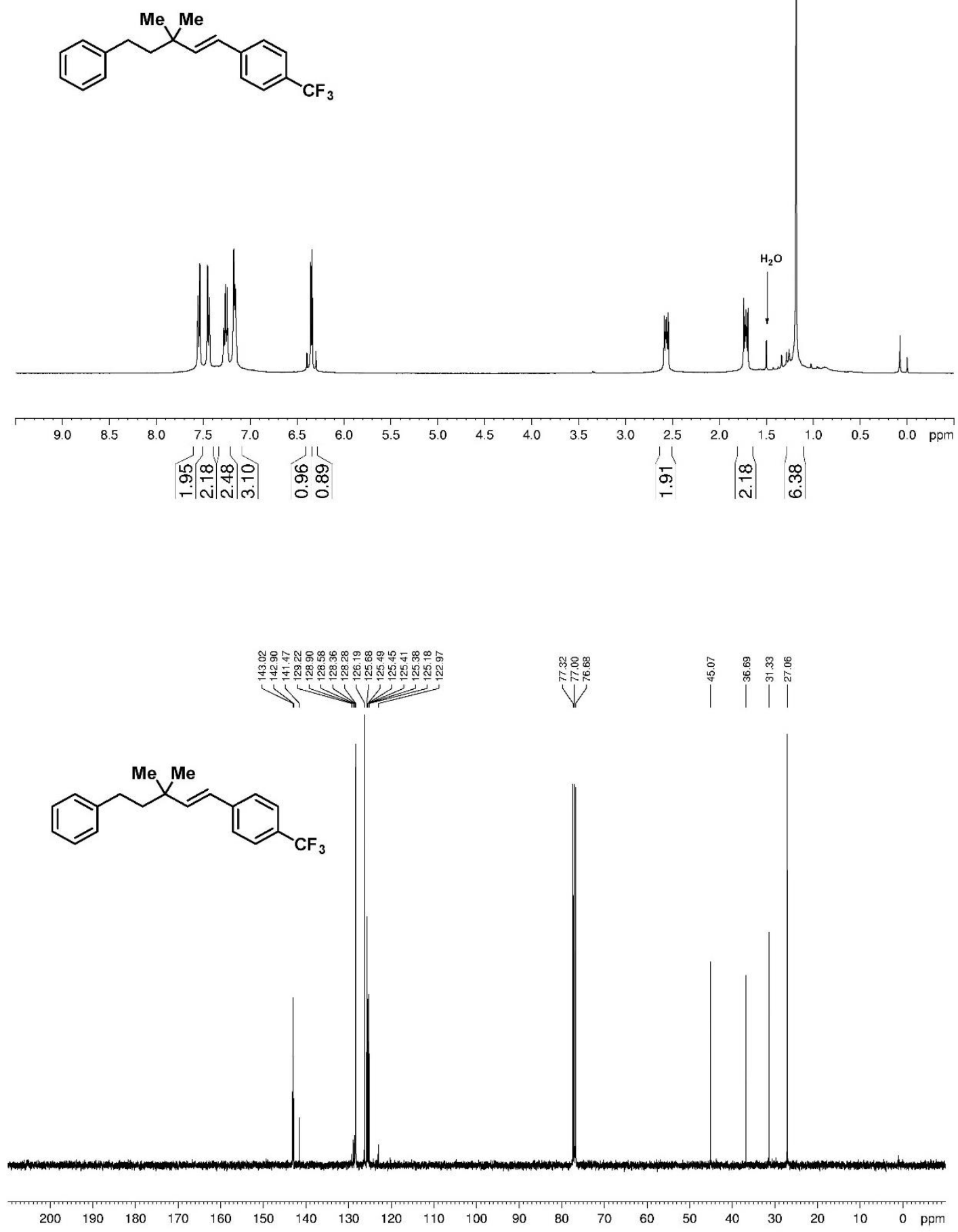

S106 
3ad; ${ }^{19}$ F NMR (376 MHz, CDCl 3 )
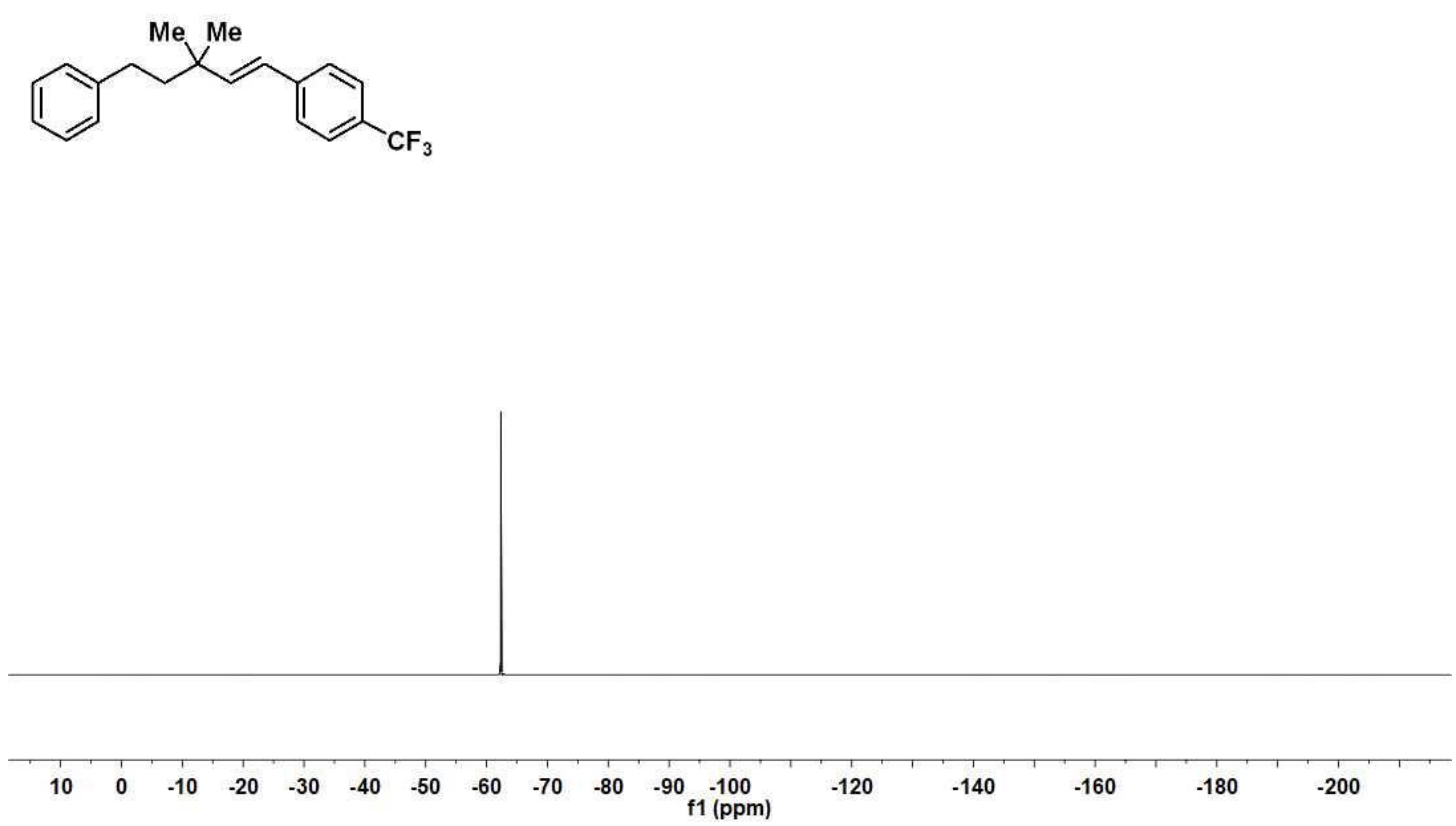

S107 
3ae; ${ }^{1} \mathrm{H}$ NMR (400 MHz, CDCl 3$) ;{ }^{13} \mathrm{C}$ NMR (100 MHz, CDCl $)$
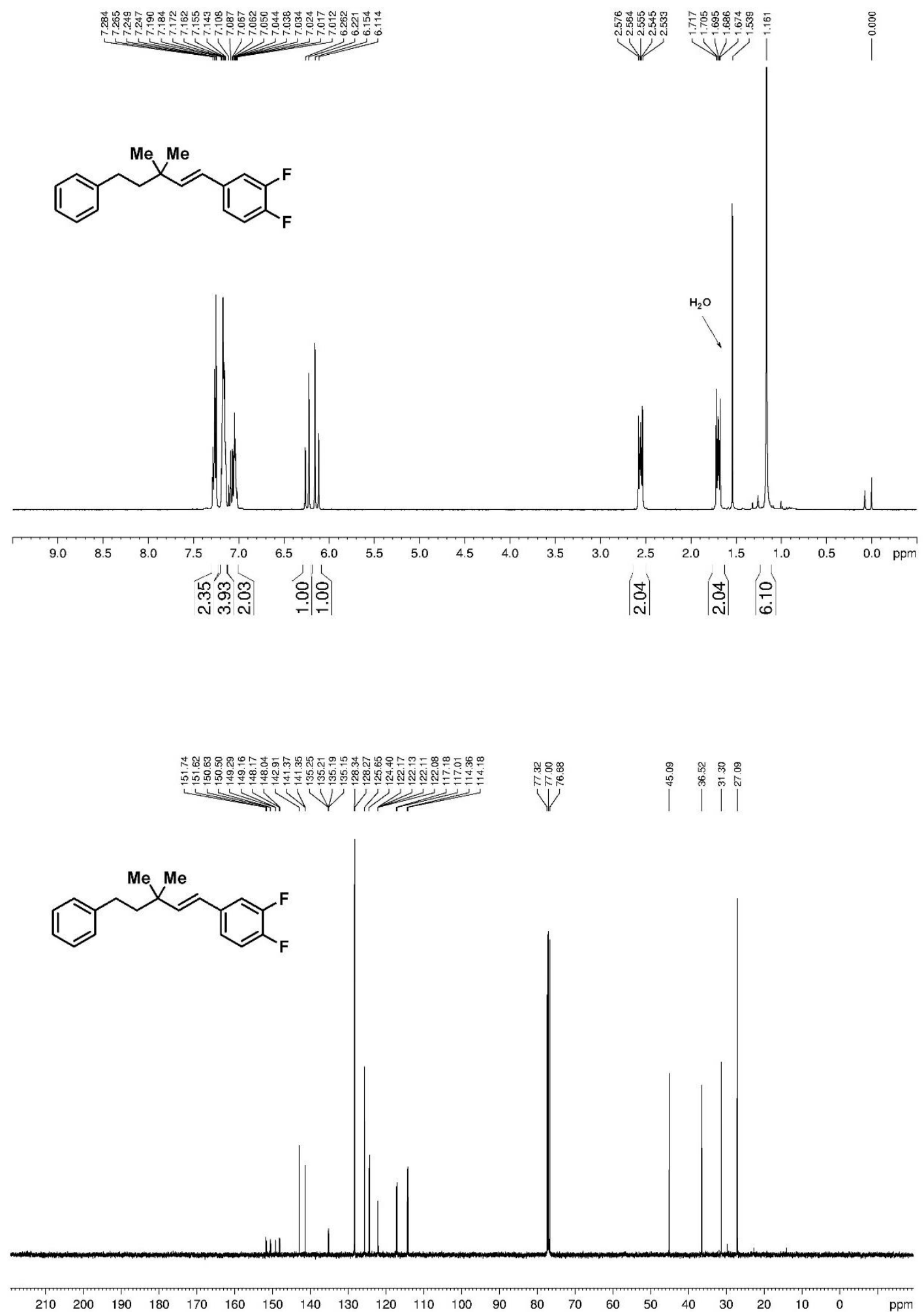
3ae; ${ }^{19}$ F NMR (376 MHz, CDCl $)$

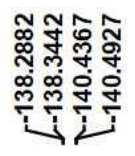
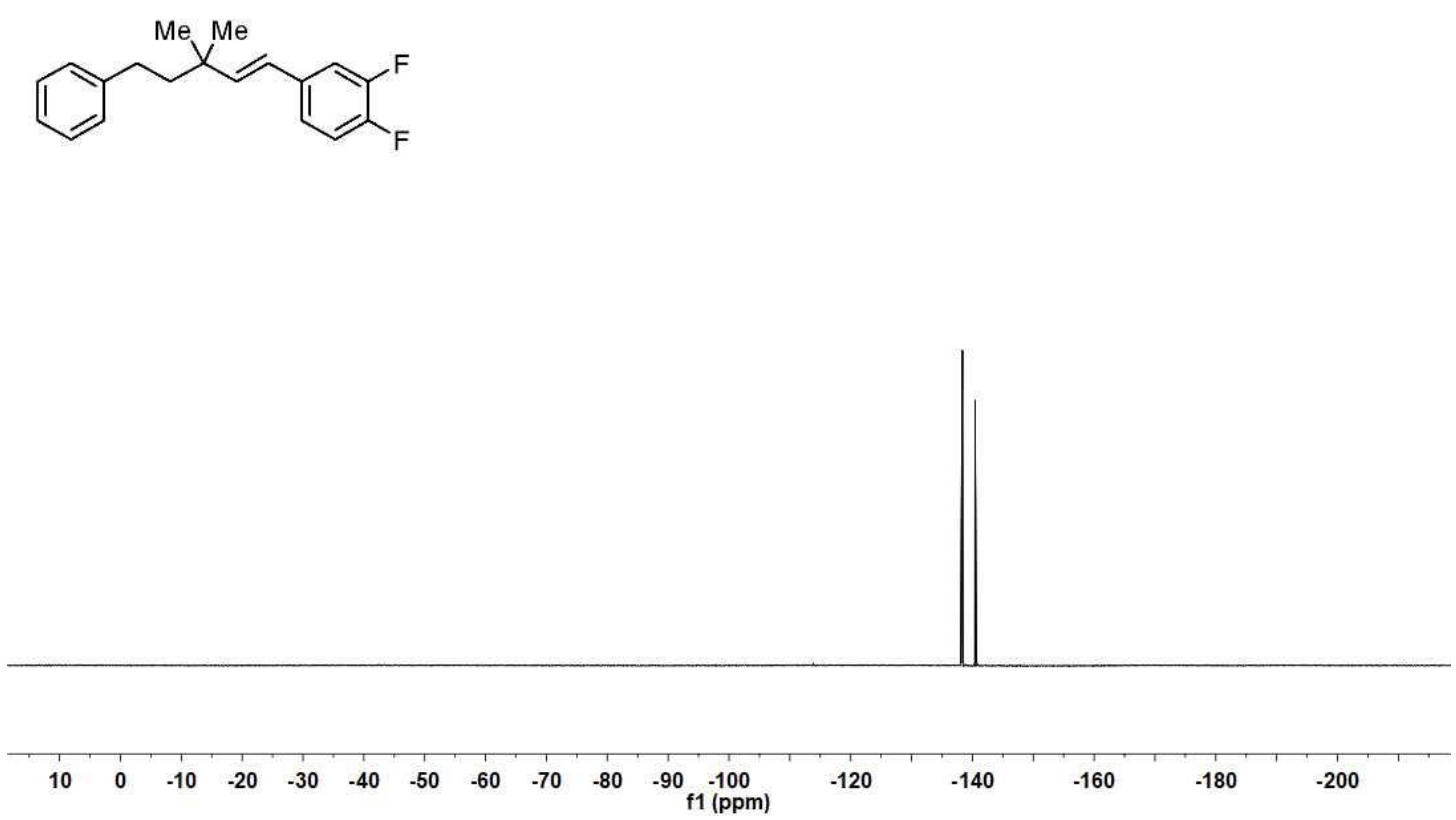

S109 
3af; ${ }^{1} \mathrm{H}$ NMR (400 MHz, CDCl$)$; ${ }^{13} \mathrm{C}$ NMR (100 MHz, CDCl $)$
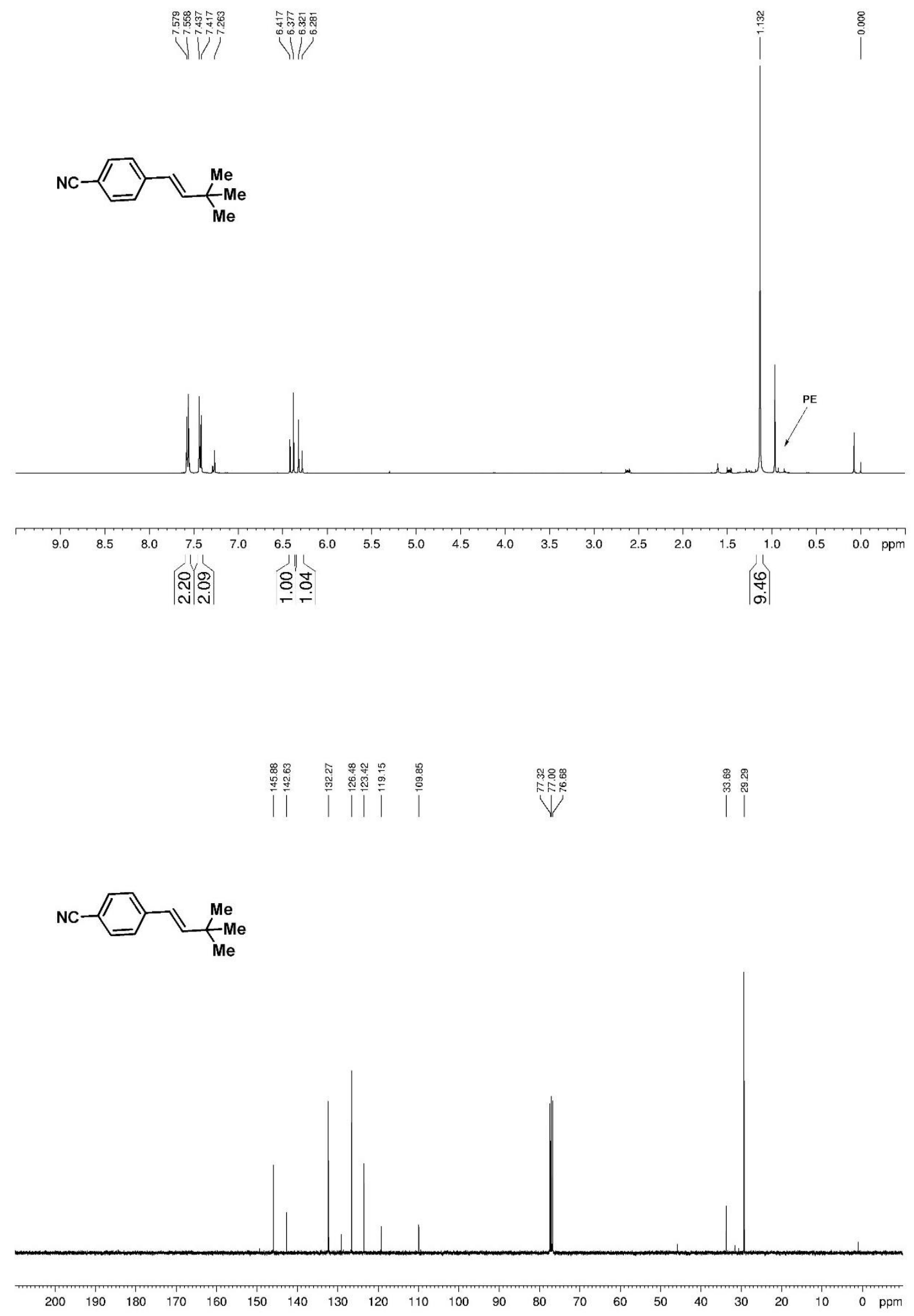
3ag; ${ }^{1} \mathrm{H}$ NMR (400 MHz, $\left.\mathrm{CDCl}_{3}\right) ;{ }^{13} \mathrm{C}$ NMR (100 $\left.\mathrm{MHz}, \mathrm{CDCl}_{3}\right)$
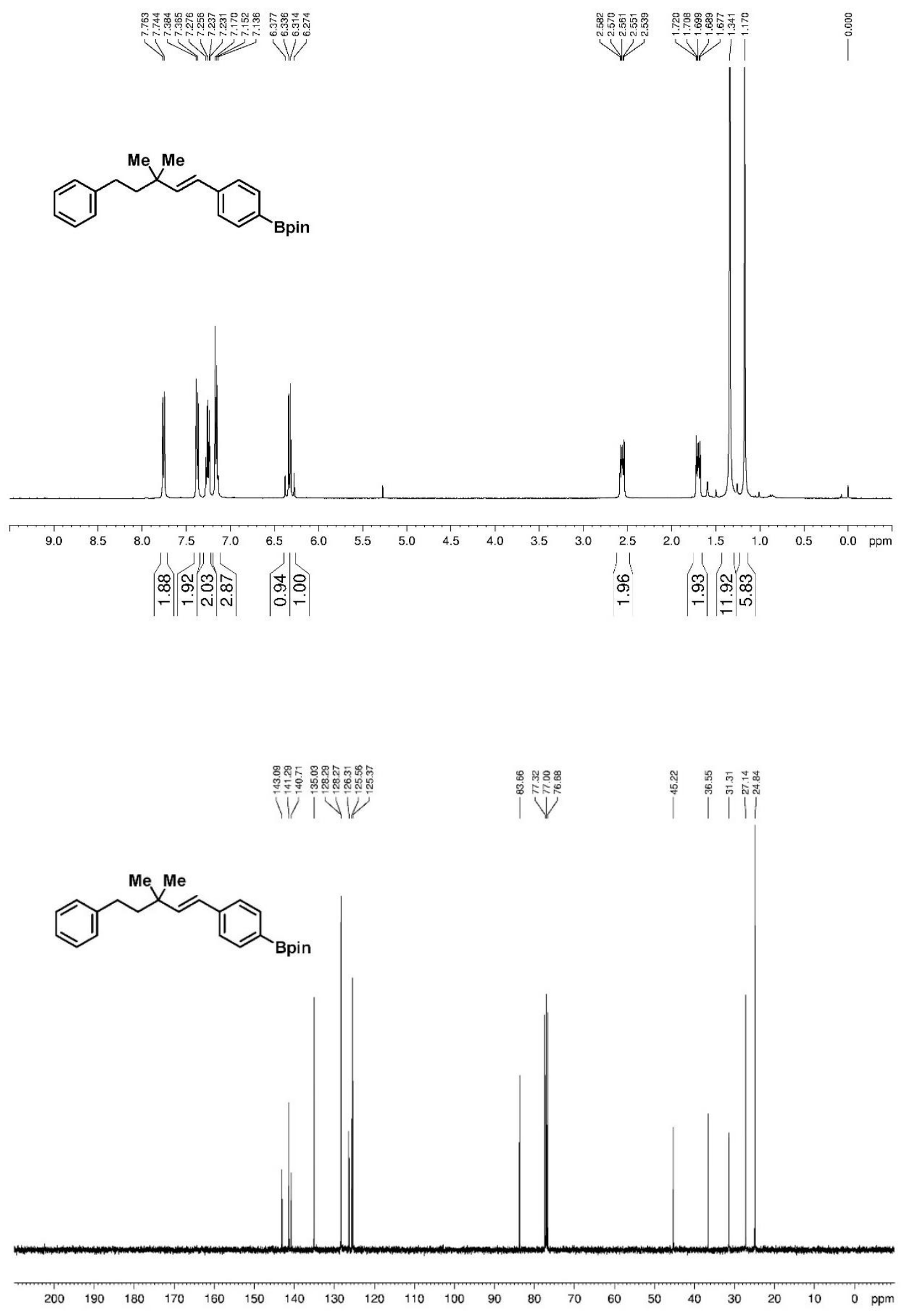

S111 
3ah; ${ }^{1} \mathrm{H}$ NMR (400 MHz, CDCl 3$) ;{ }^{13} \mathrm{C}$ NMR (100 MHz, CDCl $)$

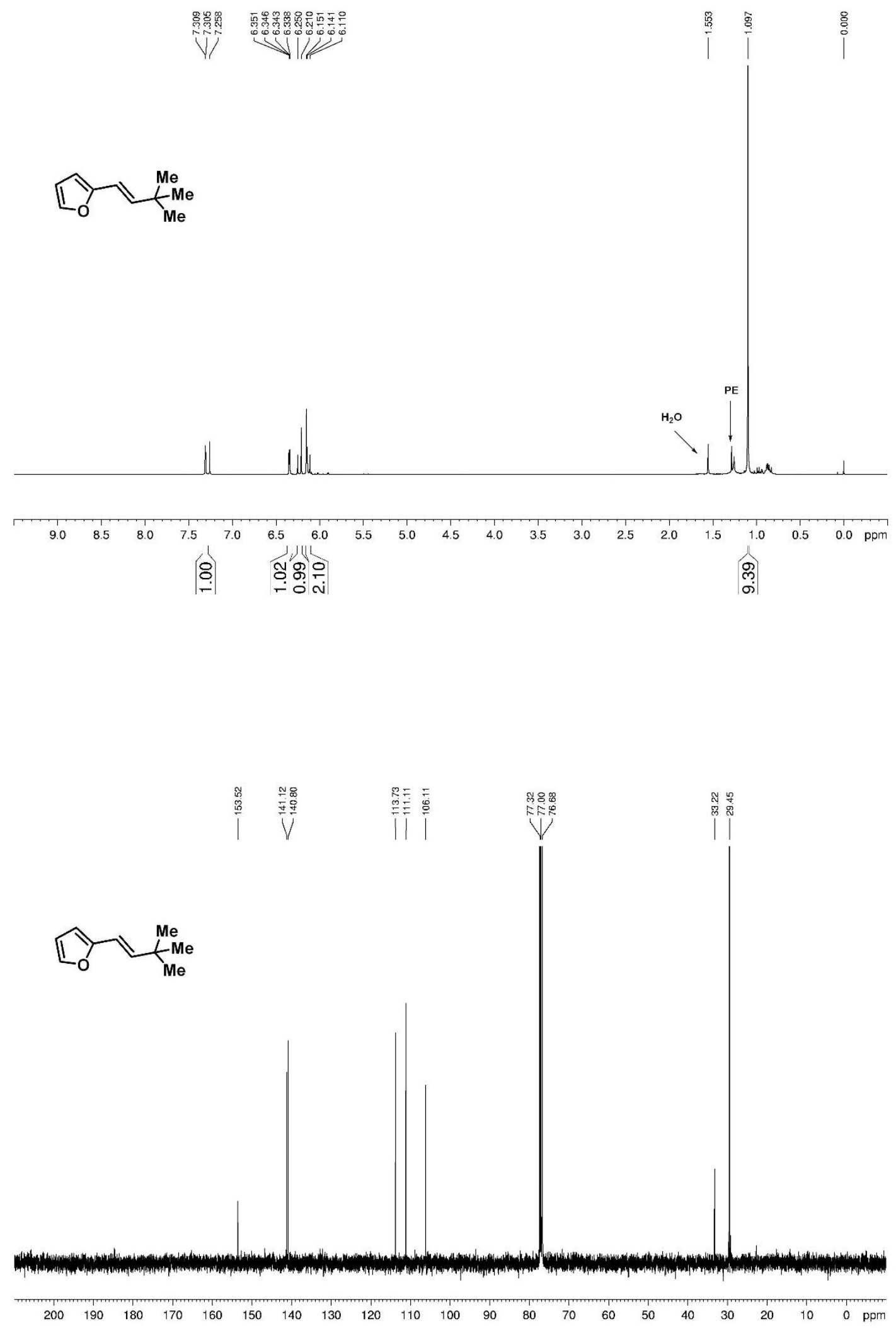


3ai; ${ }^{1} \mathrm{H}$ NMR (400 MHz, CDCl$) ;{ }^{13} \mathrm{C}$ NMR (100 MHz, CDCl 3$)$

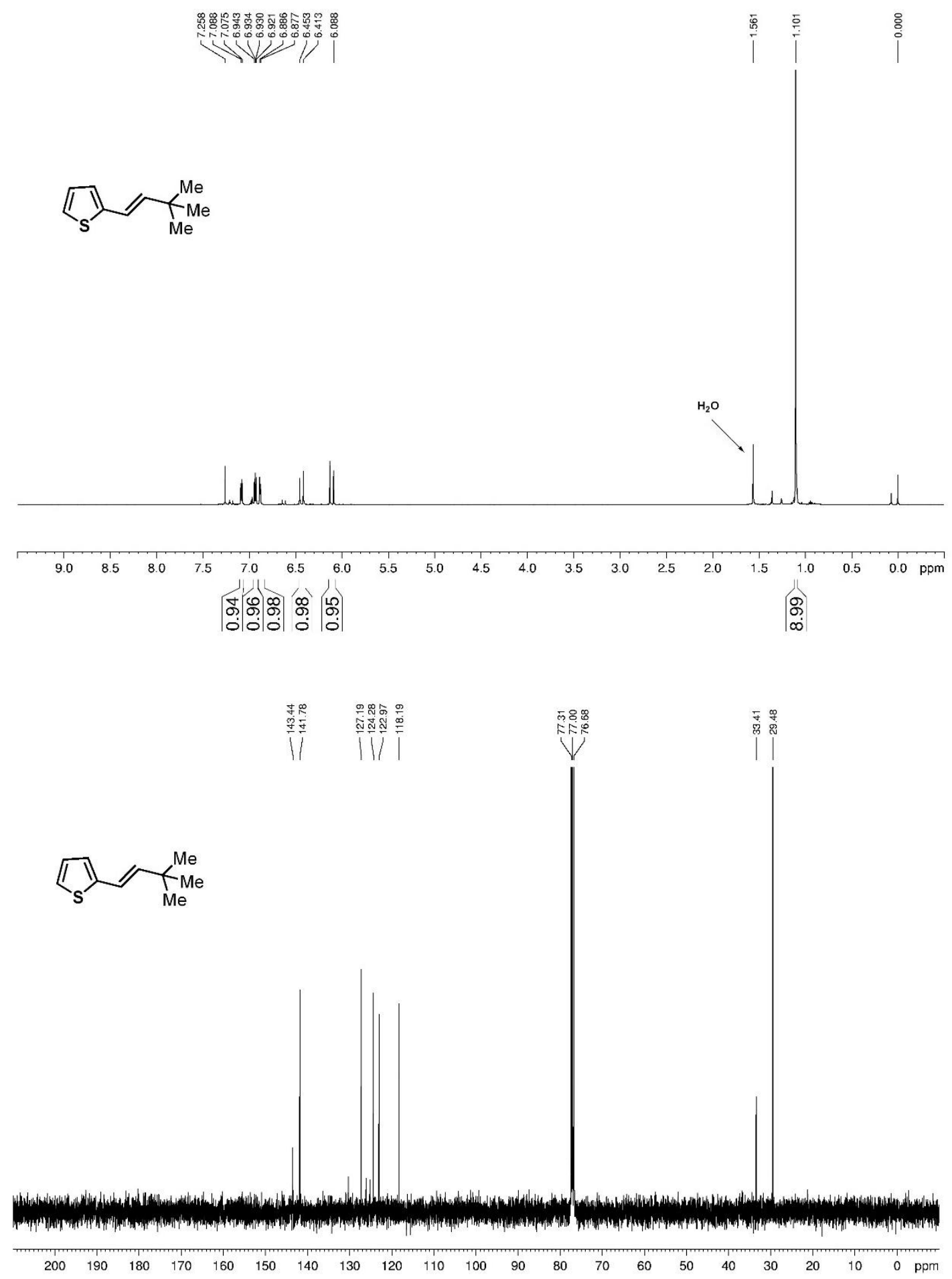


3aj; ${ }^{1} \mathrm{H}$ NMR (400 MHz, CDCl3); ${ }^{13} \mathrm{C}$ NMR (100 MHz, CDCl $)$
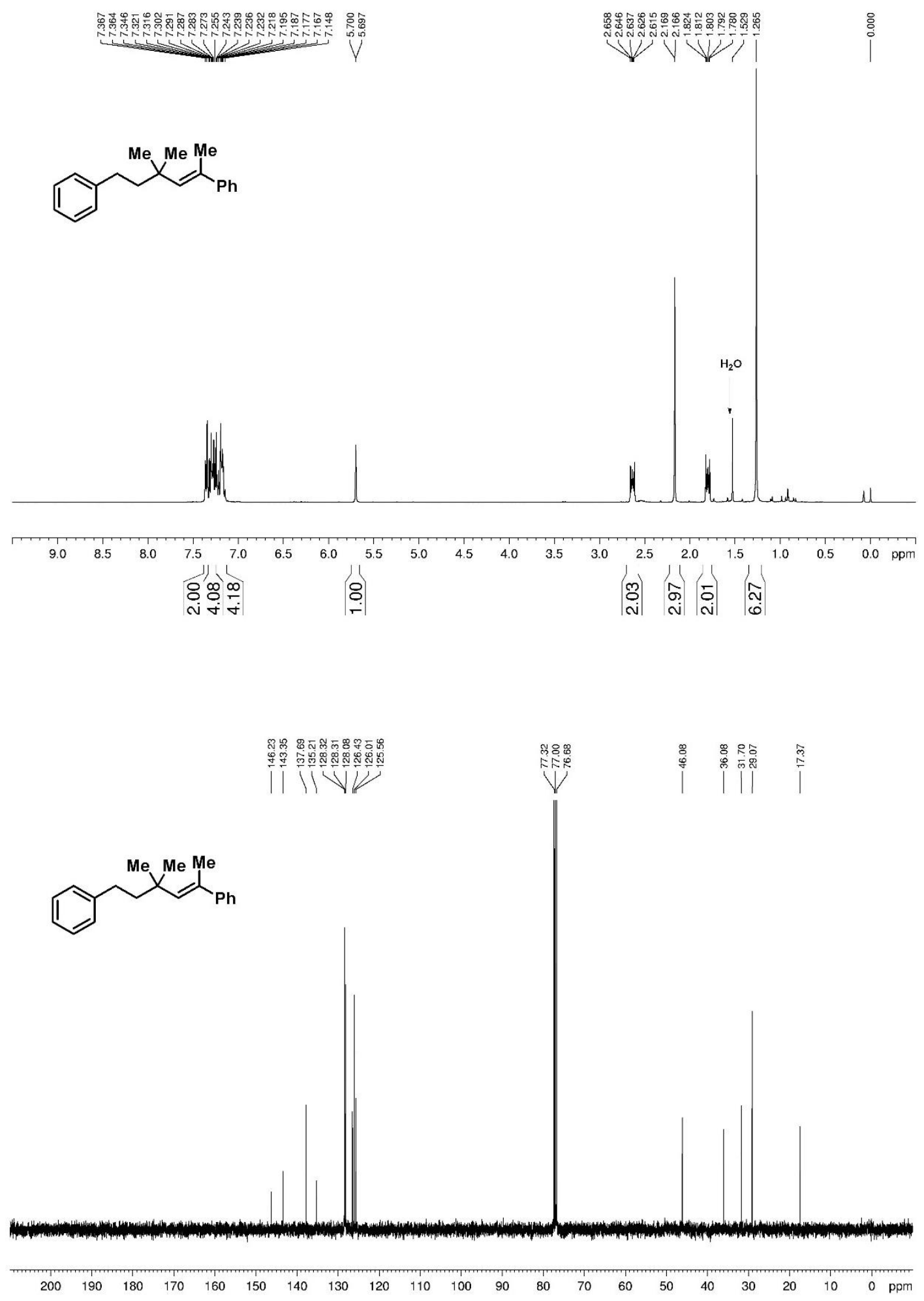

S114 
3ak; ${ }^{1} \mathrm{H}$ NMR (400 MHz, $\left.\mathrm{CDCl}_{3}\right) ;{ }^{13} \mathrm{C}$ NMR (100 MHz, CDCl$)$
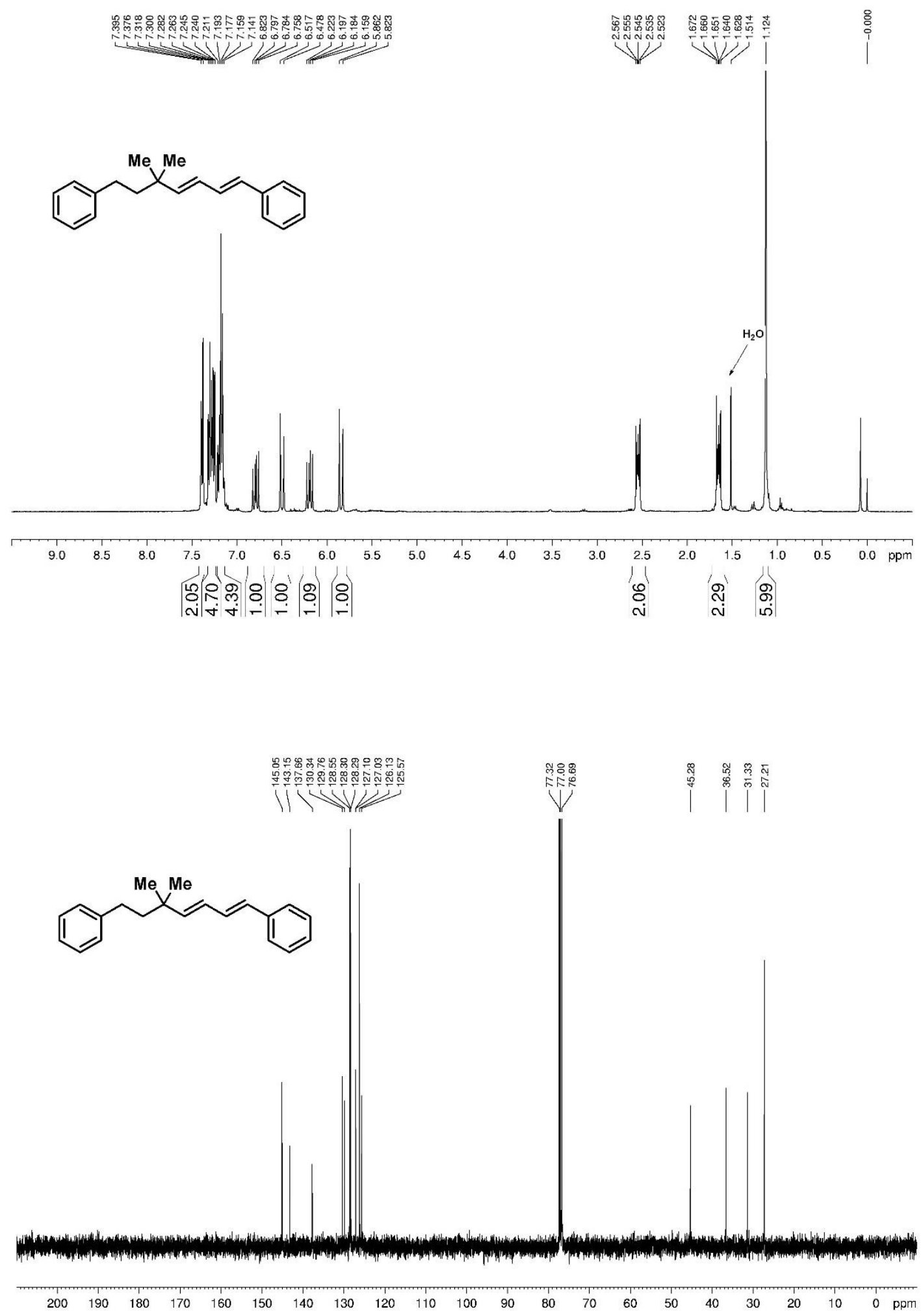
3al; ${ }^{1} \mathrm{H}$ NMR (400 MHz, CDCl$) ;{ }^{13} \mathrm{C}$ NMR (100 MHz, CDCl 3$)$

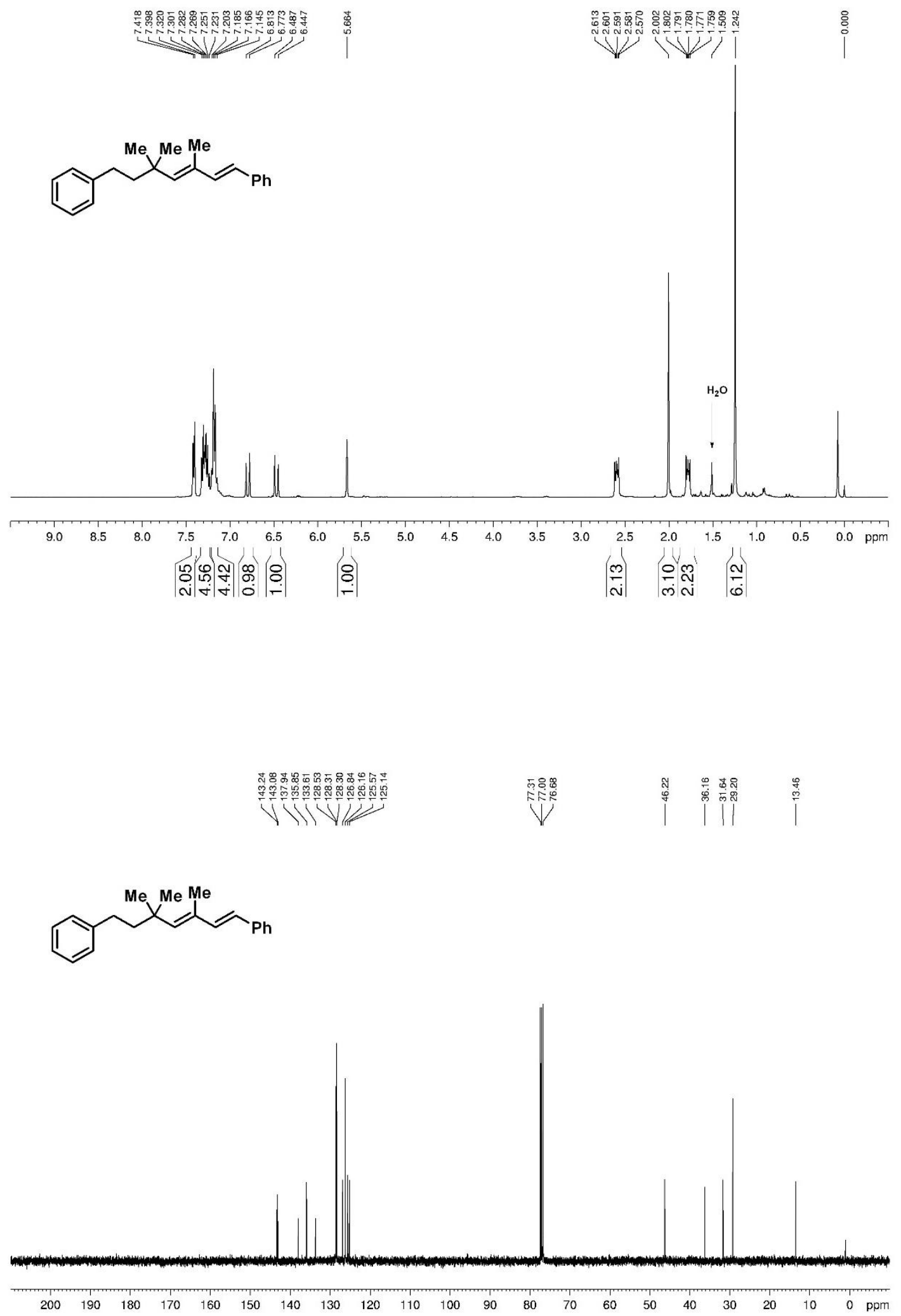


3am; ${ }^{1} \mathrm{H}$ NMR (400 MHz, $\left.\mathrm{CDCl}_{3}\right) ;{ }^{13} \mathrm{C}$ NMR $\left(100 \mathrm{MHz}, \mathrm{CDCl}_{3}\right)$

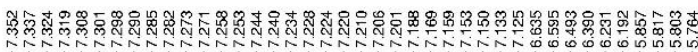

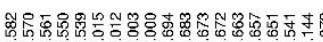

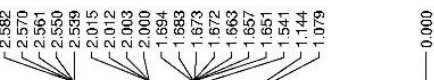

$\overbrace{\text { II }}^{\mathrm{Me}} \mathrm{P}_{\mathrm{Mh}}^{\mathrm{Me}}$
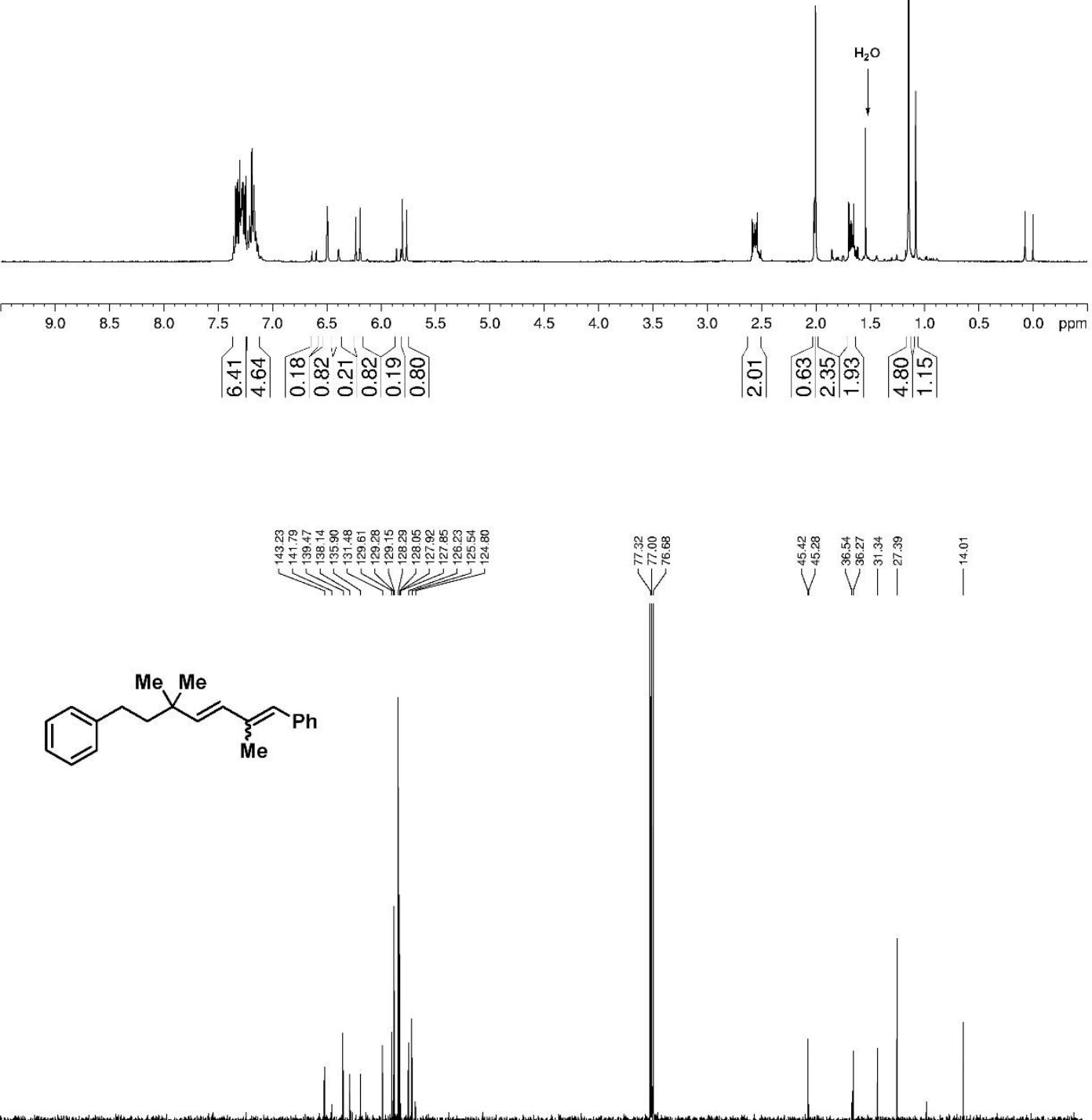

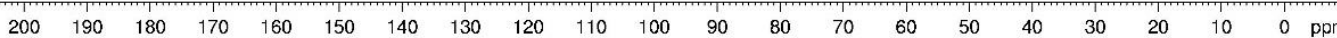


3an; ${ }^{1} \mathrm{H}$ NMR (400 MHz, $\left.\mathrm{CDCl}_{3}\right) ;{ }^{13} \mathrm{C}$ NMR (100 MHz, CDCl$)$

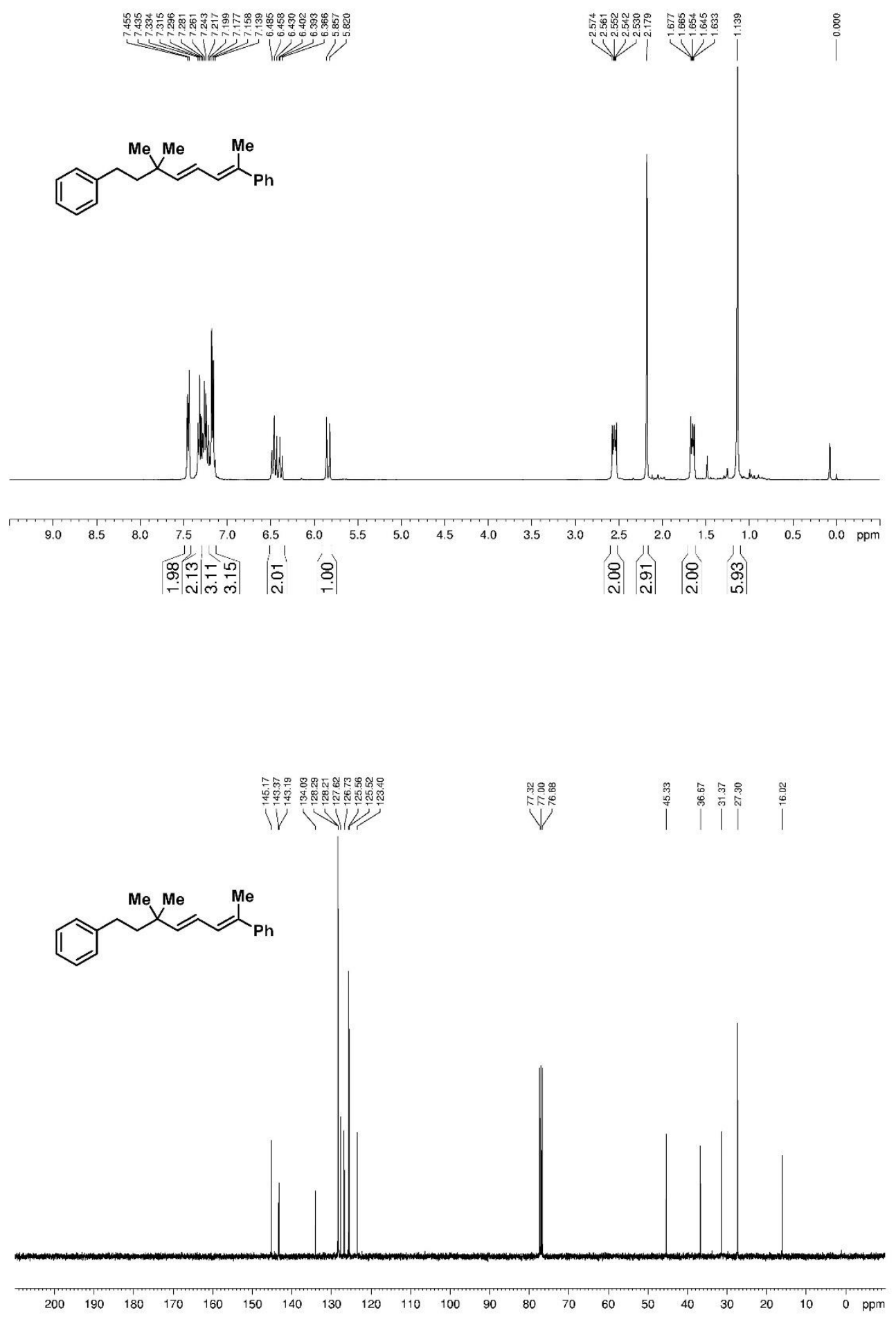


3ao; ${ }^{1} \mathrm{H}$ NMR (400 MHz, $\left.\mathrm{CDCl}_{3}\right) ;{ }^{13} \mathrm{C}$ NMR (100 $\left.\mathrm{MHz}, \mathrm{CDCl}_{3}\right)$

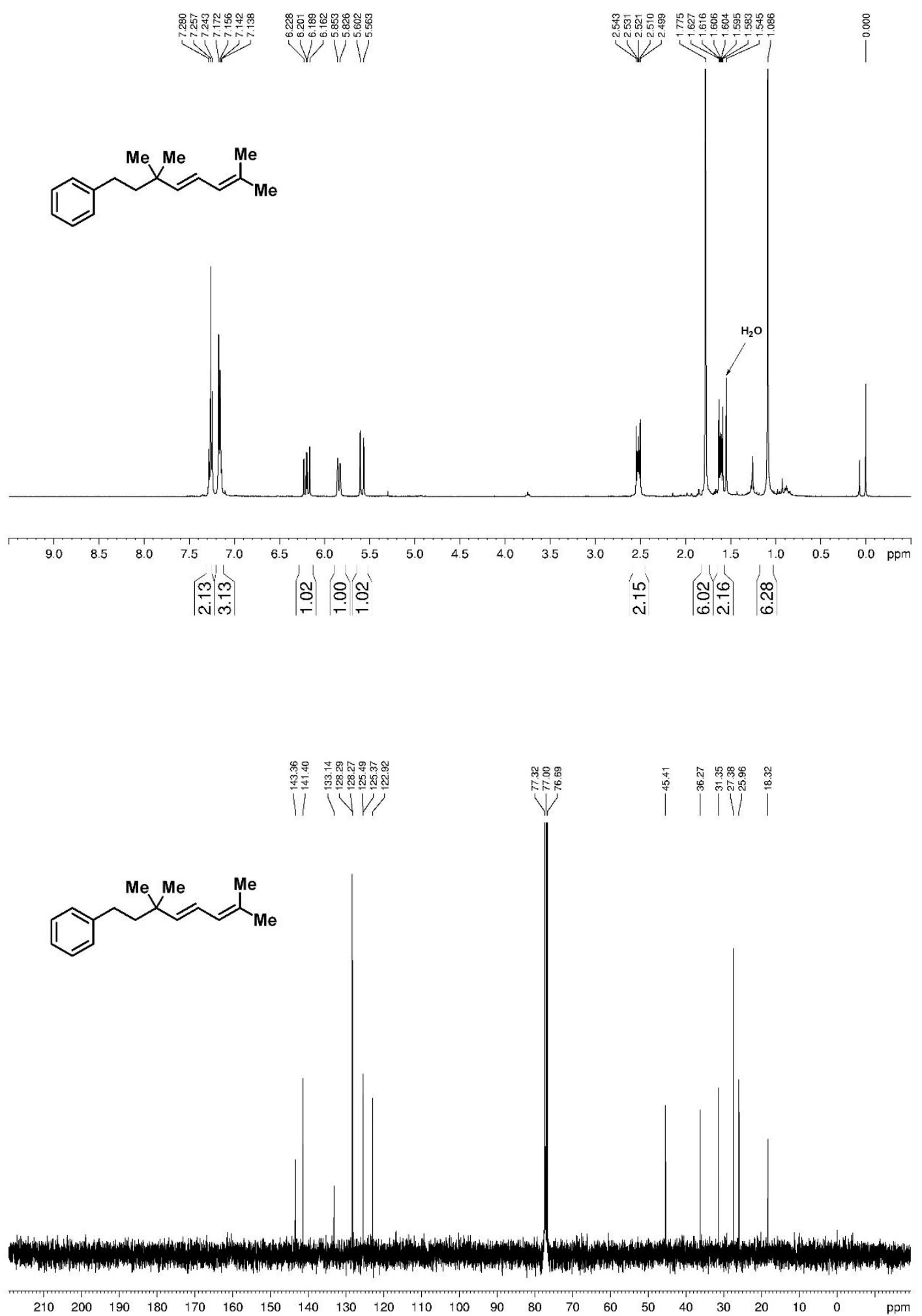


3ap; ${ }^{1} \mathrm{H}$ NMR (400 MHz, $\left.\mathrm{CDCl}_{3}\right) ;{ }^{13} \mathrm{C}$ NMR (100 MHz, $\left.\mathrm{CDCl}_{3}\right)$

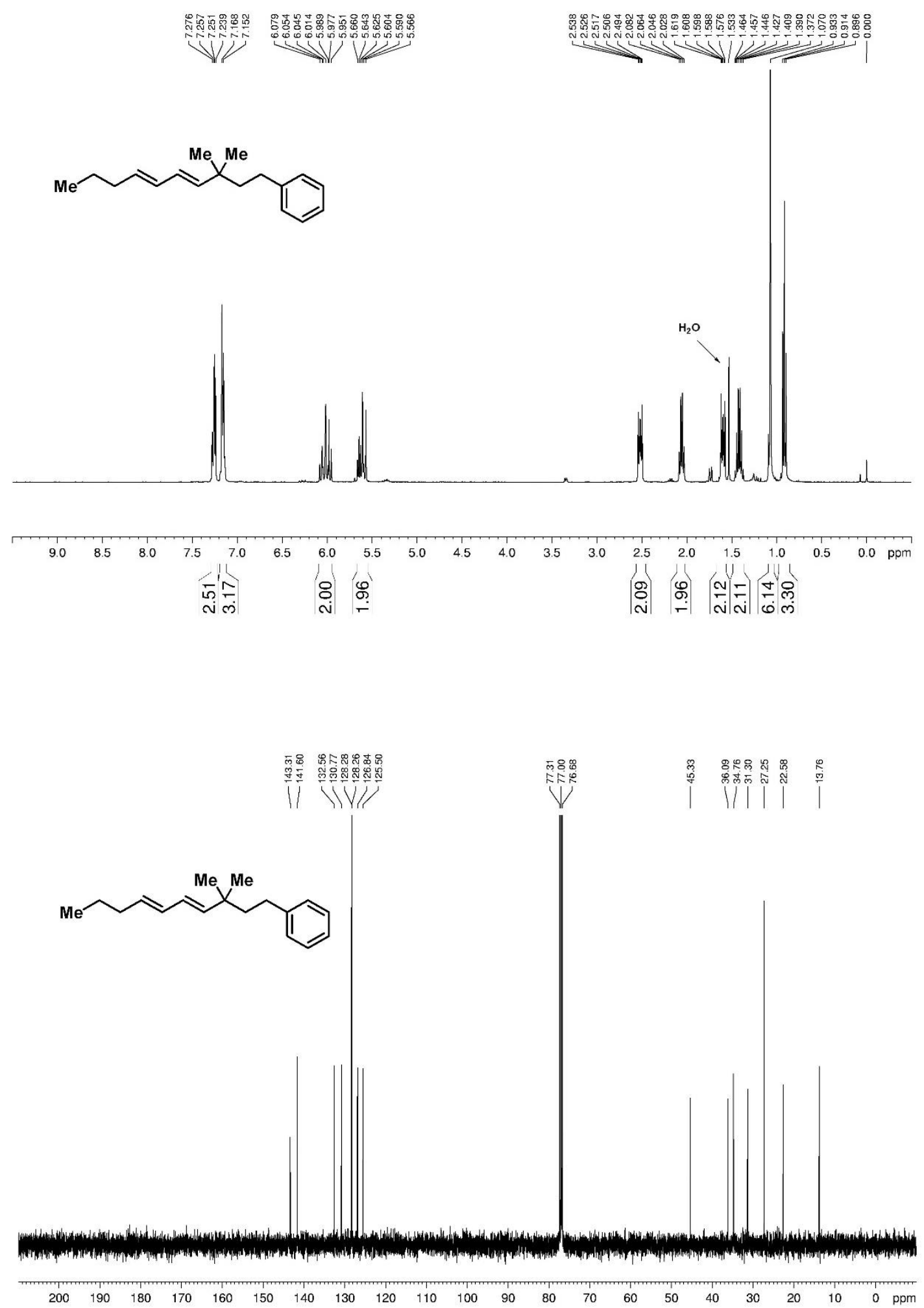


3aq; ${ }^{1} \mathrm{H}$ NMR (400 MHz, $\left.\mathrm{CDCl}_{3}\right) ;{ }^{13} \mathrm{C}$ NMR (100 MHz, $\left.\mathrm{CDCl}_{3}\right)$
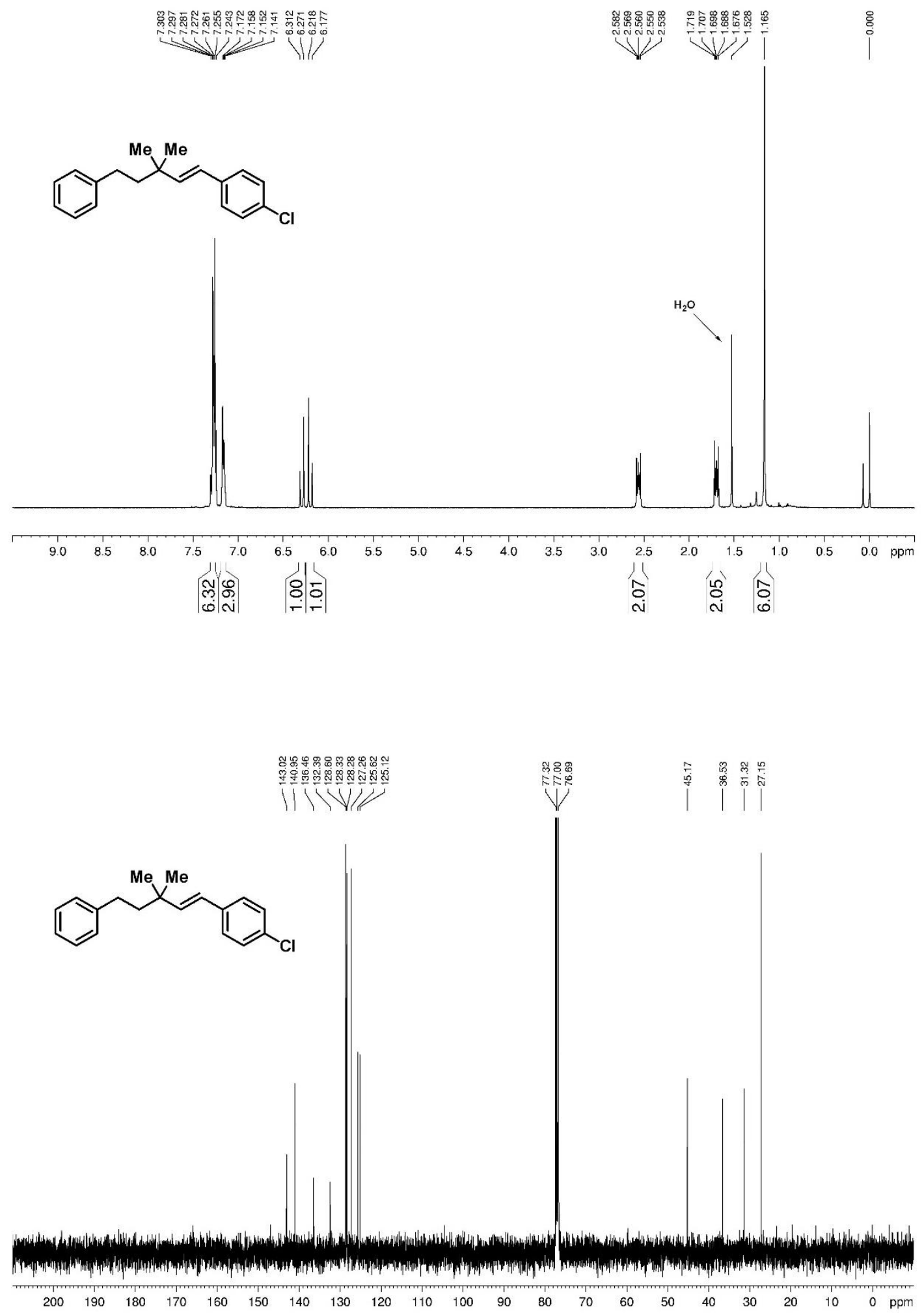
3ar; ${ }^{1} \mathrm{H}$ NMR (400 MHz, CDCl $)$; ${ }^{13} \mathrm{C}$ NMR (100 MHz, CDCl $)$

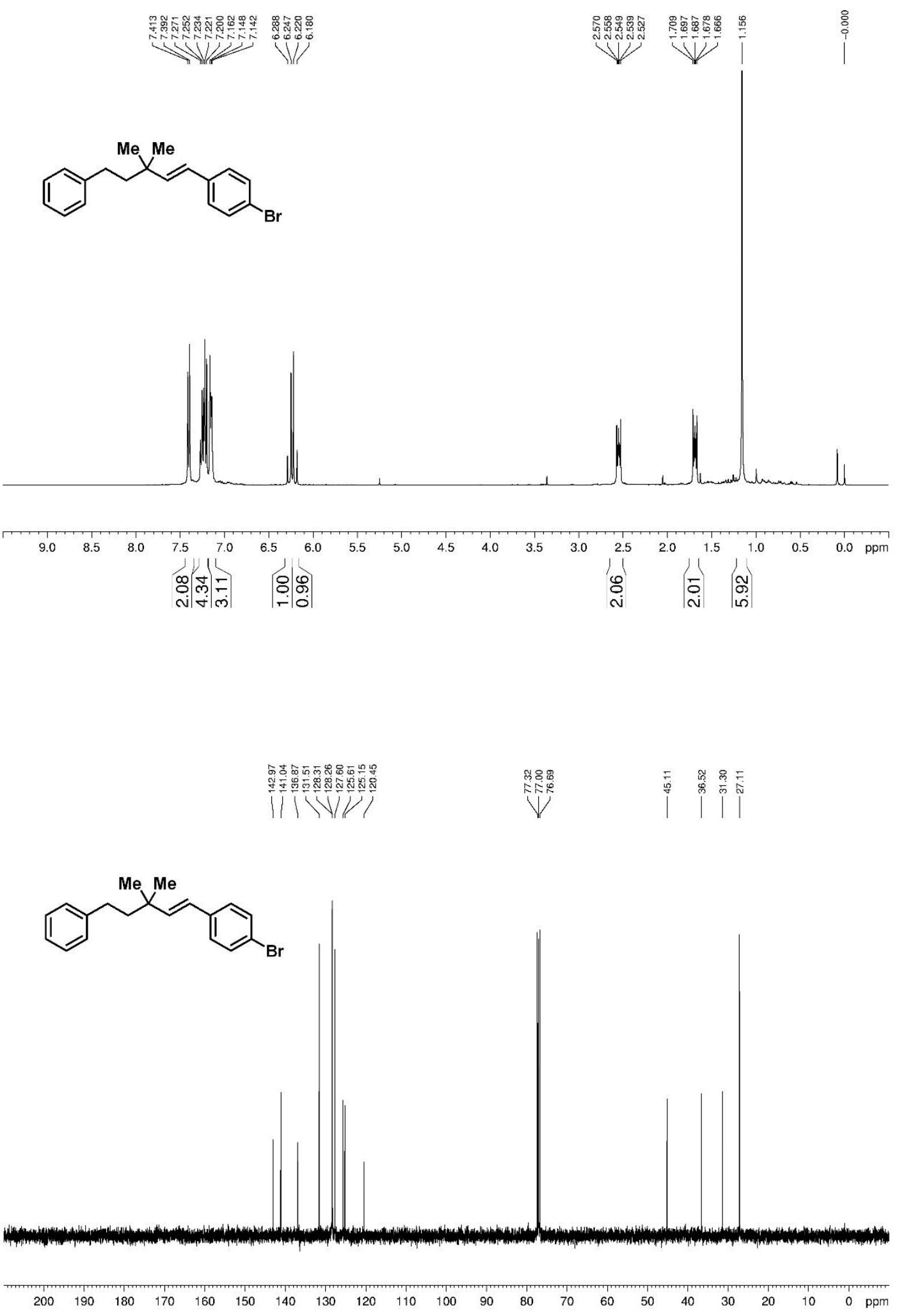


3as; ${ }^{1} \mathrm{H}$ NMR (400 $\left.\mathrm{MHz}, \mathrm{CDCl}_{3}\right) ;{ }^{13} \mathrm{C}$ NMR (100 $\left.\mathrm{MHz}, \mathrm{CDCl}_{3}\right)$
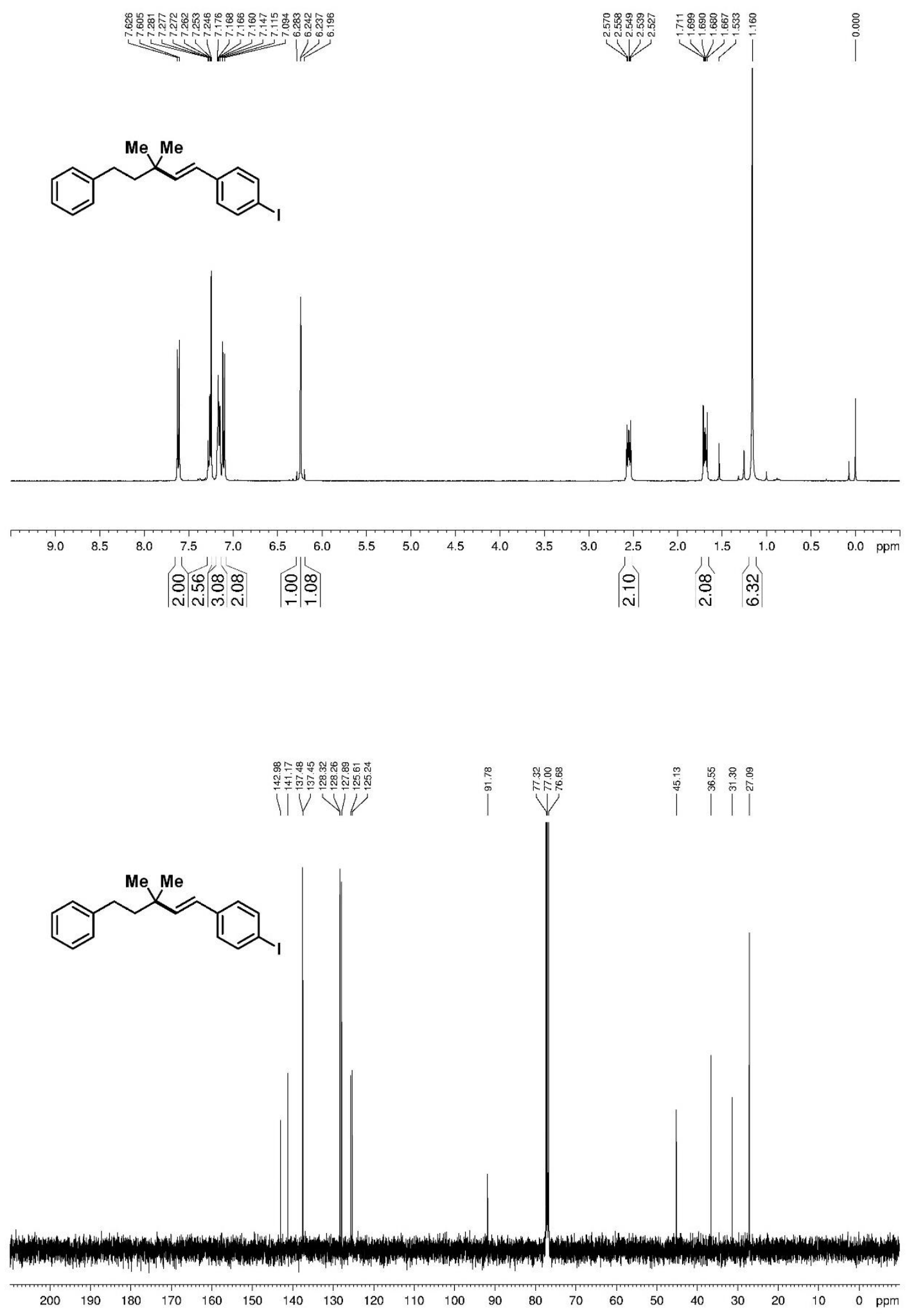

S123 
3at; ${ }^{1} \mathrm{H}$ NMR (600 MHz, CDCl $) ;{ }^{13} \mathrm{C}$ NMR (150 MHz, CDCl 3$)$
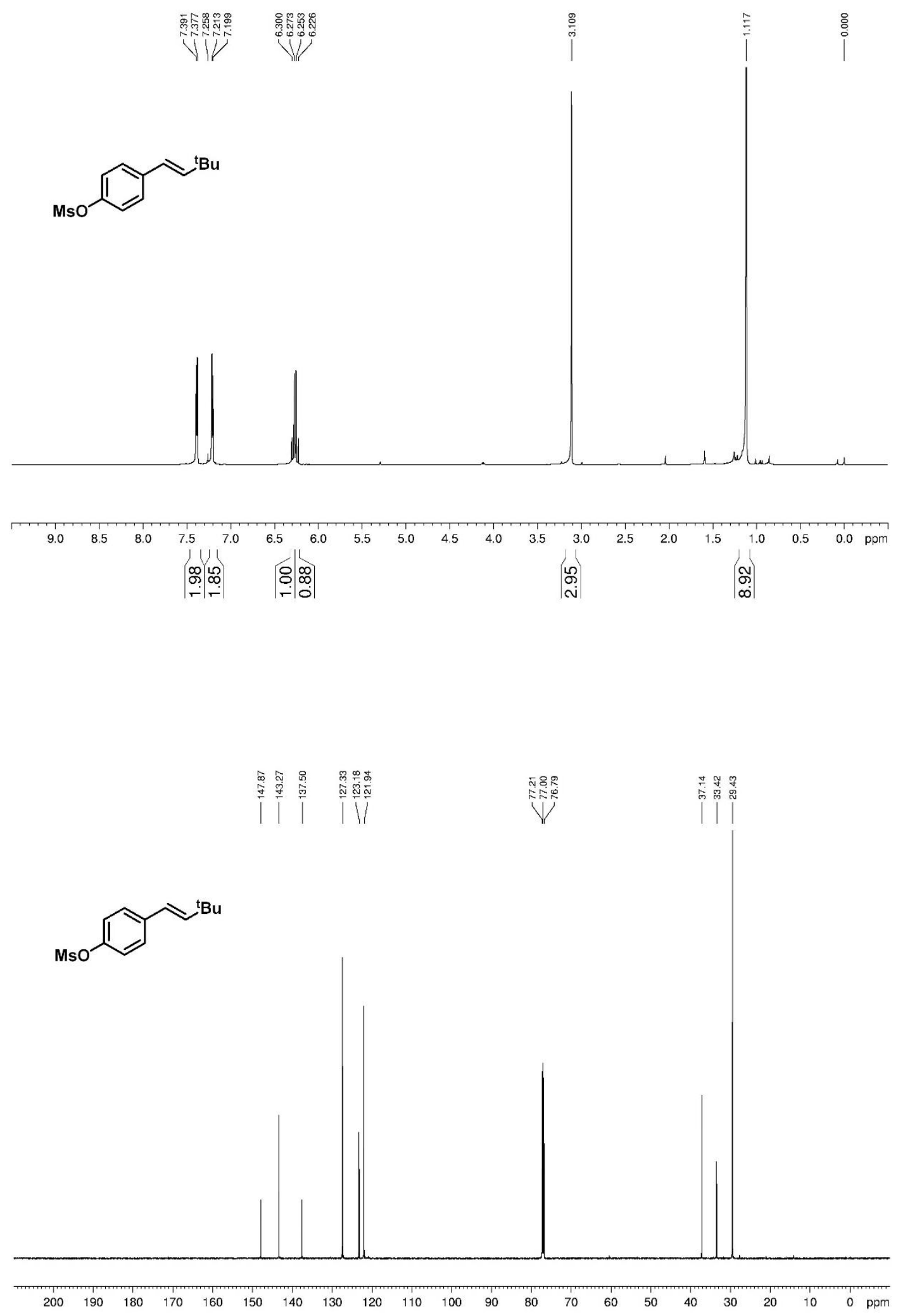
3au; ${ }^{1} \mathrm{H}$ NMR (600 MHz, CDCl 3$) ;{ }^{13} \mathrm{C}$ NMR (150 MHz, CDCl $)$
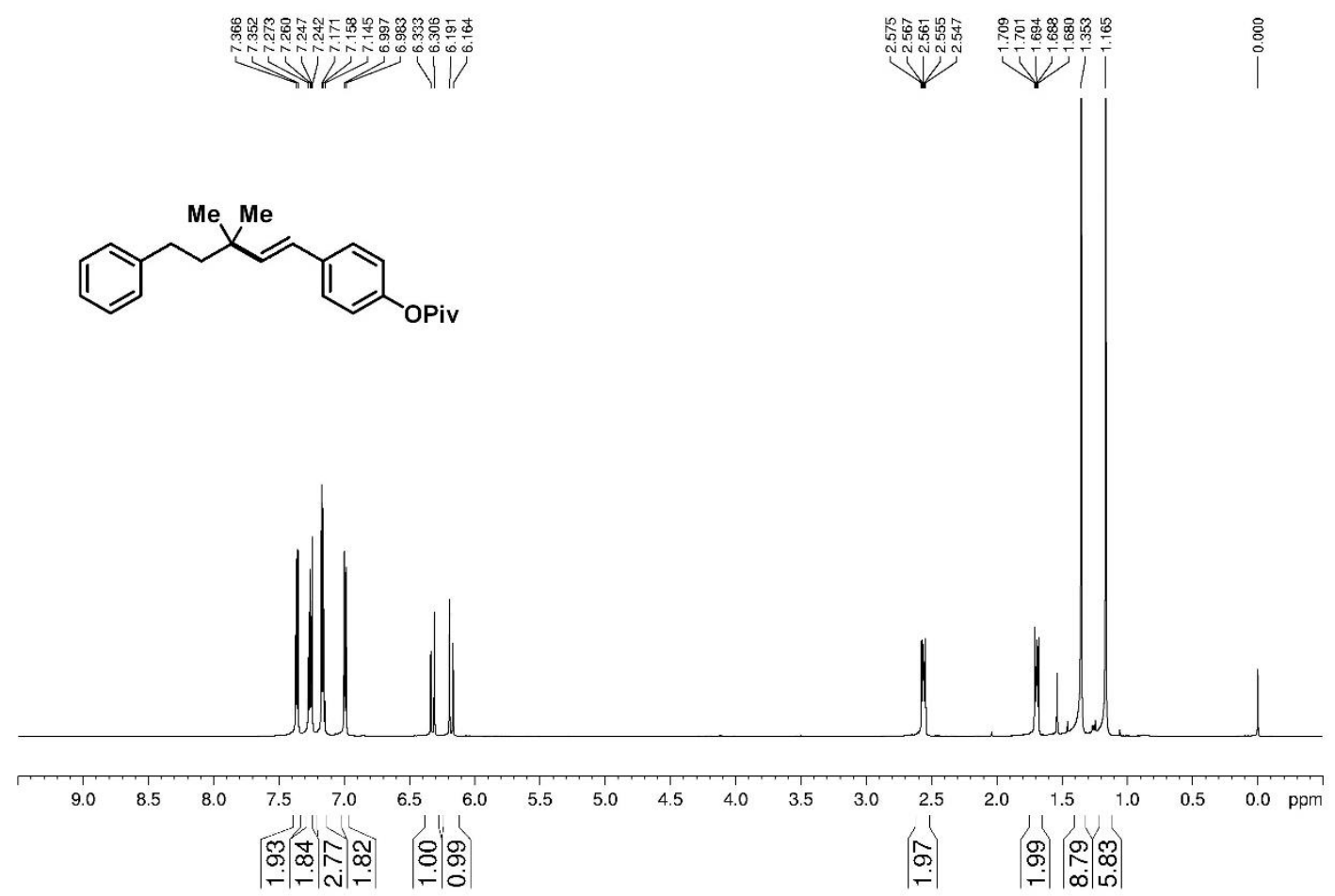

$\left.\right|^{2}$

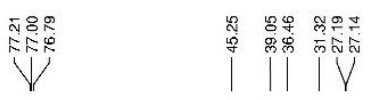
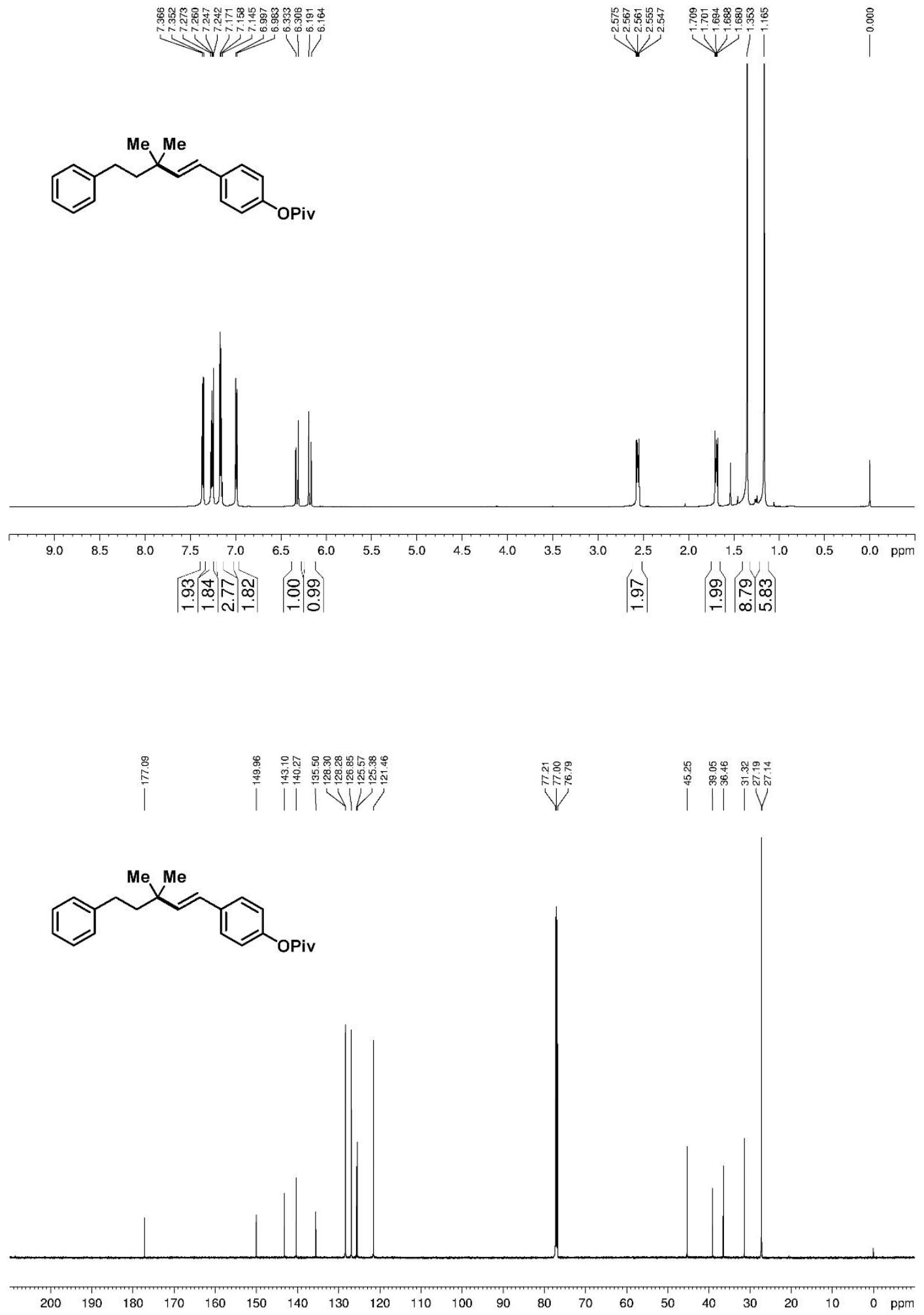
3av; ${ }^{1} \mathrm{H}$ NMR (600 MHz, $\left.\mathrm{CDCl}_{3}\right) ;{ }^{13} \mathrm{C}$ NMR (150 MHz, $\left.\mathrm{CDCl}_{3}\right)$
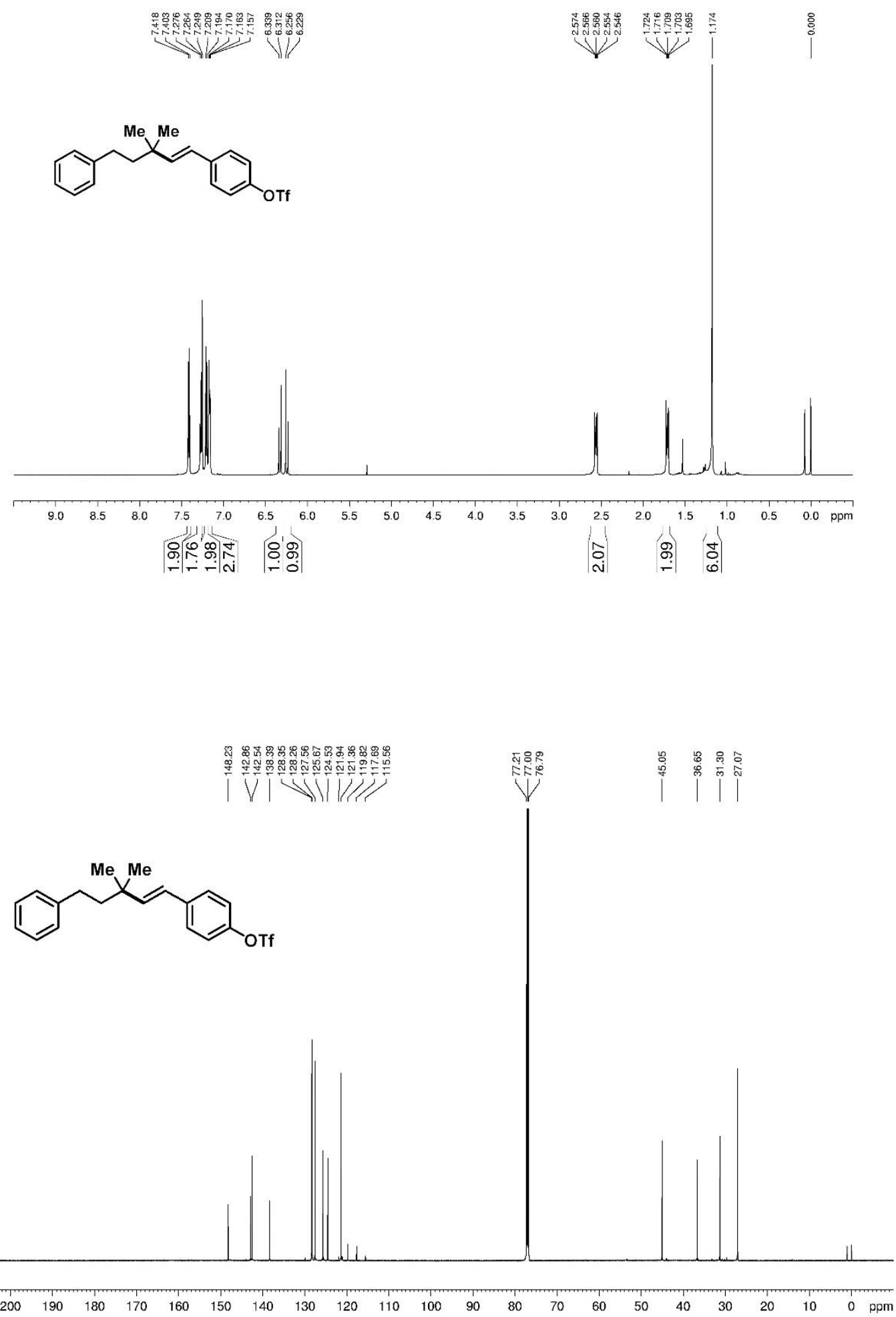
3av; ${ }^{19}$ F NMR (376 MHz, CDCl $)$

$\underset{\substack{i \\ \text { i }}}{\mathfrak{i}}$
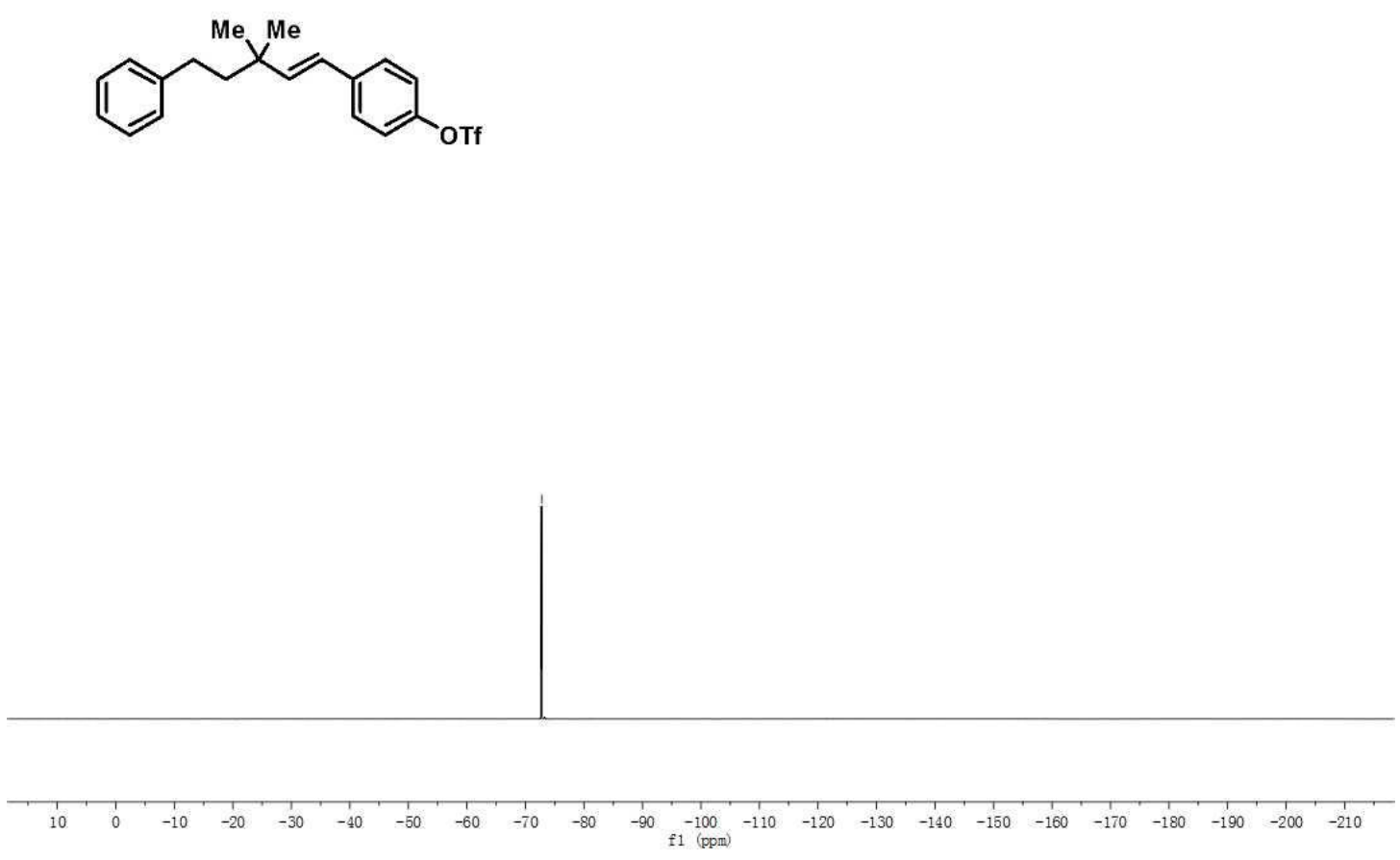

S127 
3aw; ${ }^{1} \mathrm{H}$ NMR (400 MHz, $\left.\mathrm{CDCl}_{3}\right) ;{ }^{13} \mathrm{C}$ NMR (100 MHz, $\left.\mathrm{CDCl}_{3}\right)$

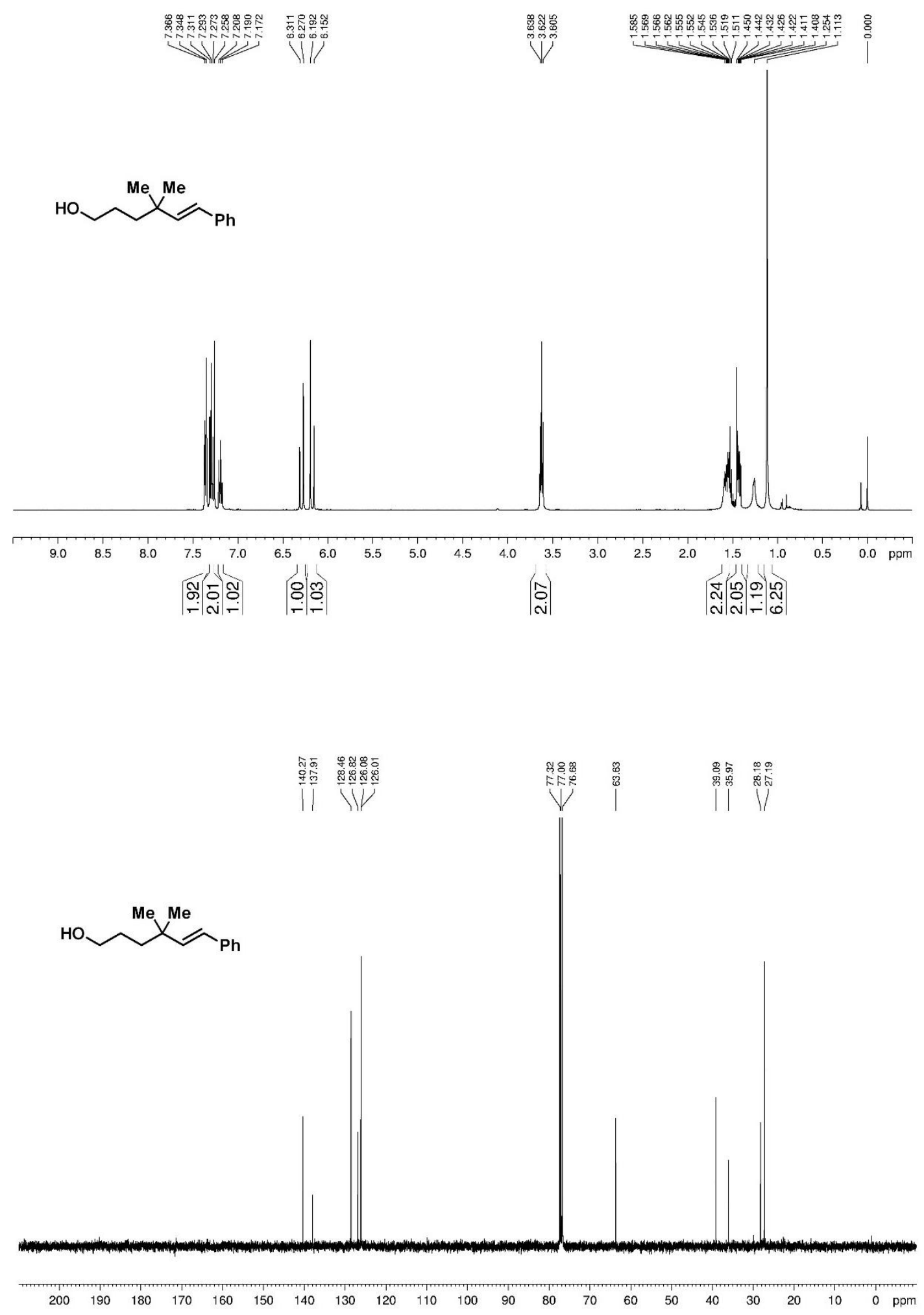


3ax; ${ }^{1} \mathrm{H}$ NMR (400 MHz, CDCl 3$) ;{ }^{13} \mathrm{C}$ NMR (100 MHz, CDCl 3$)$
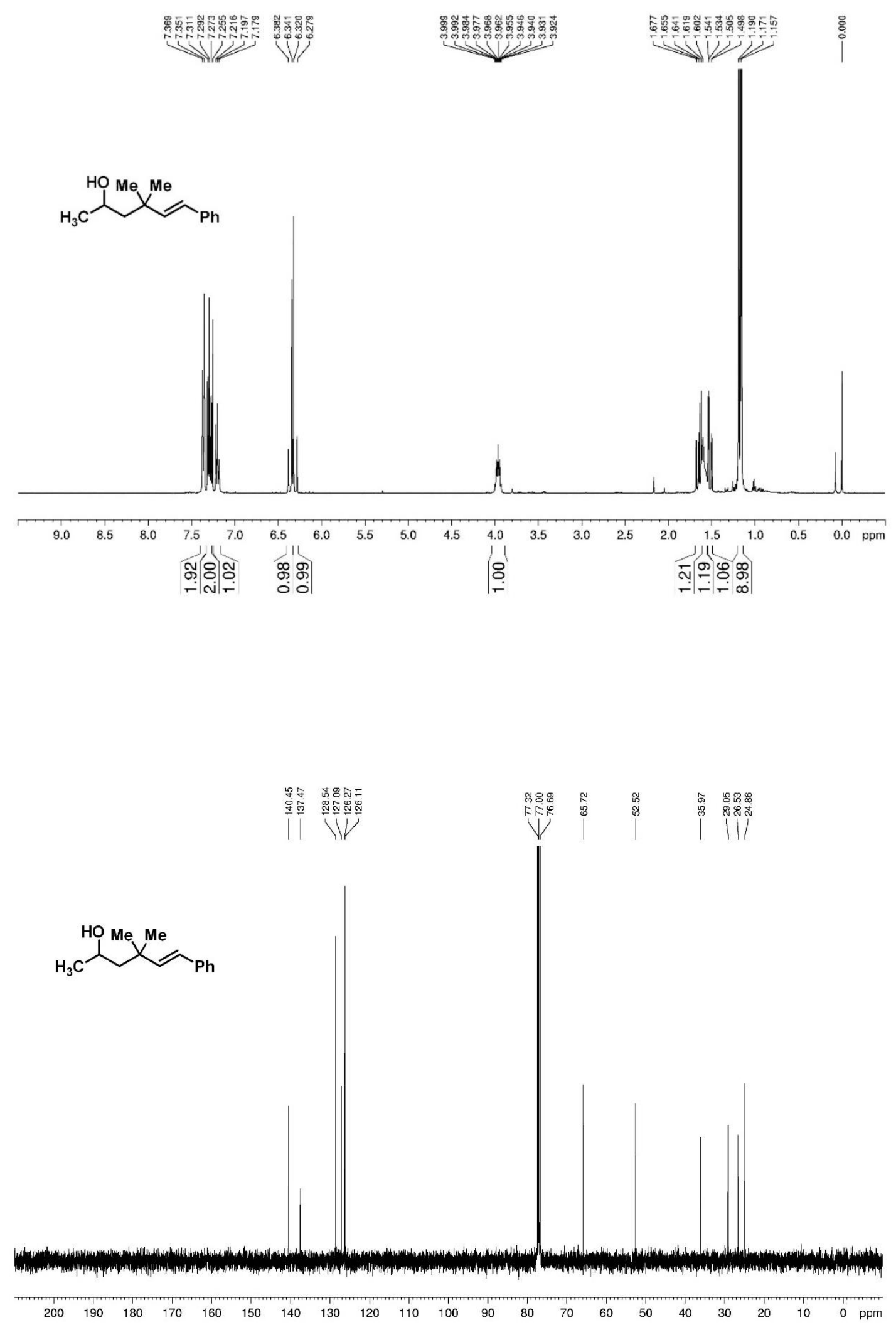
3ay; ${ }^{1} \mathrm{H}$ NMR (600 MHz, $\left.\mathrm{CDCl}_{3}\right) ;{ }^{13} \mathrm{C}$ NMR (150 MHz, $\left.\mathrm{CDCl}_{3}\right)$

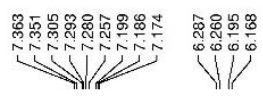

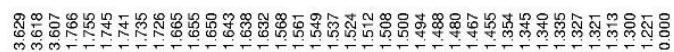
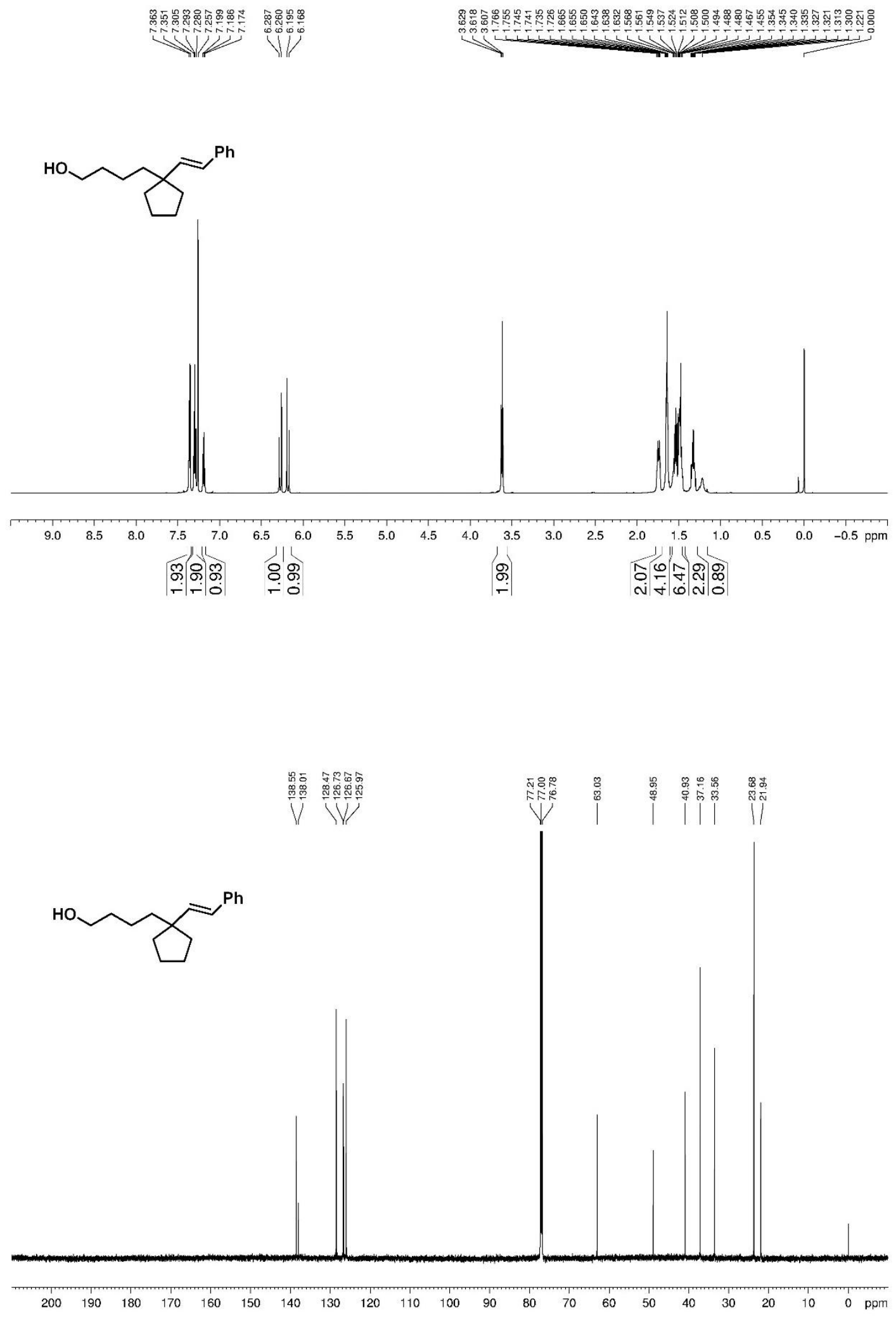
3az; ${ }^{1} \mathrm{H}$ NMR (600 MHz, CDCl $) ;{ }^{13} \mathrm{C}$ NMR (150 MHz, CDCl $)$

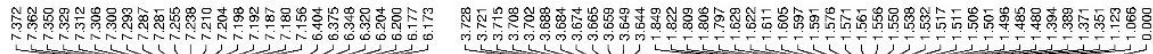<smiles>CC1(C=CP)CCC(O)CC1</smiles>

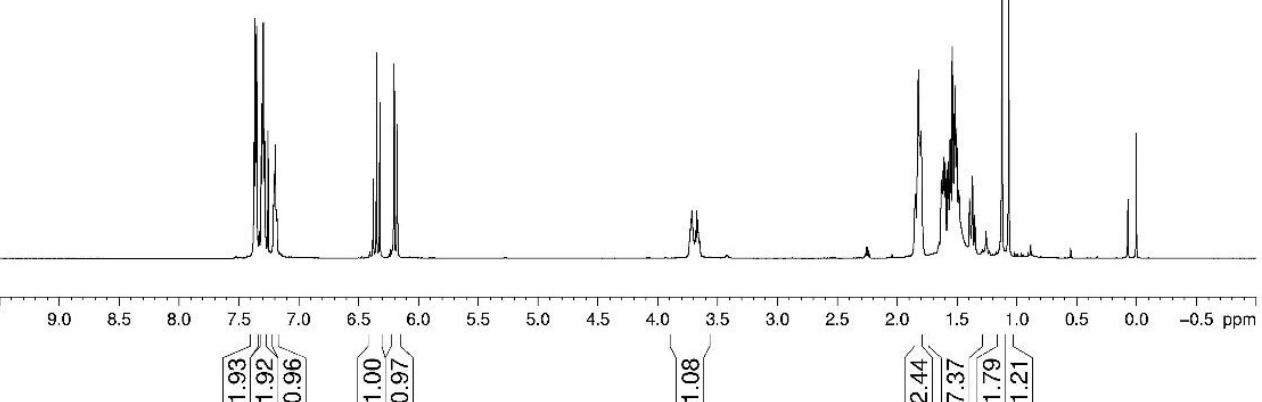<smiles>CC1(/C=C/c2ccccc2)CCC(O)CC1</smiles>

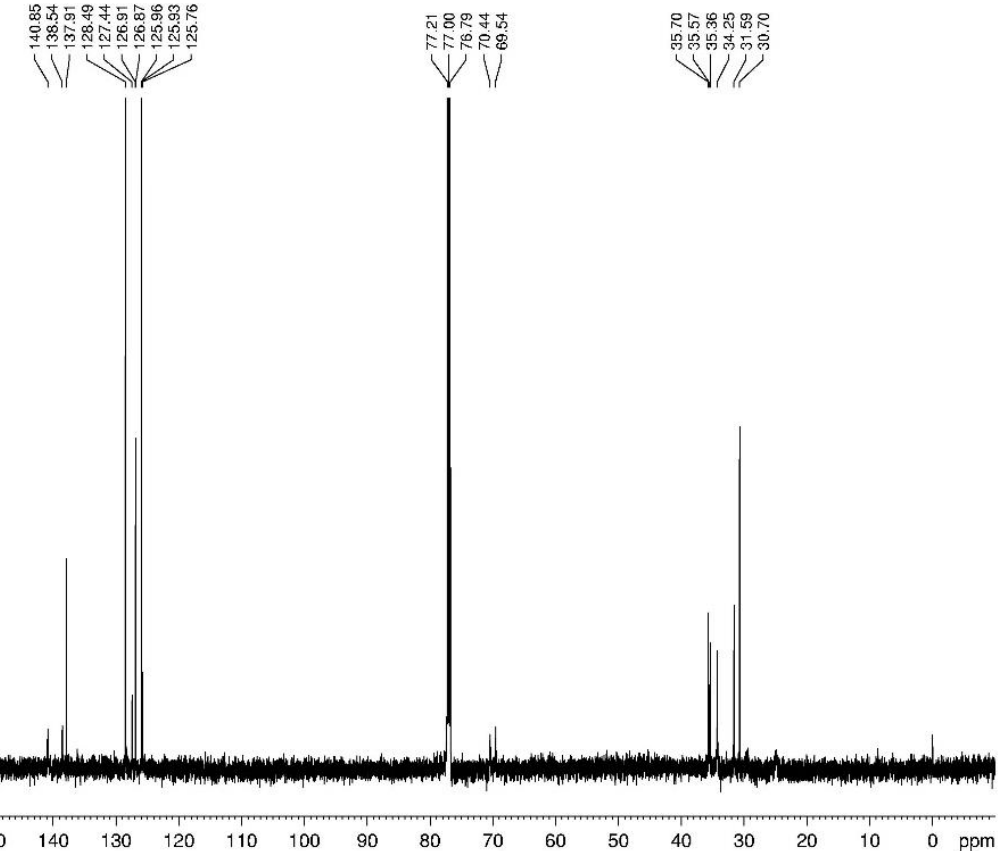


3ba; ${ }^{1} \mathrm{H}$ NMR (400 MHz, $\left.\mathrm{CDCl}_{3}\right) ;{ }^{13} \mathrm{C}$ NMR (150 MHz, CDCl 3$)$
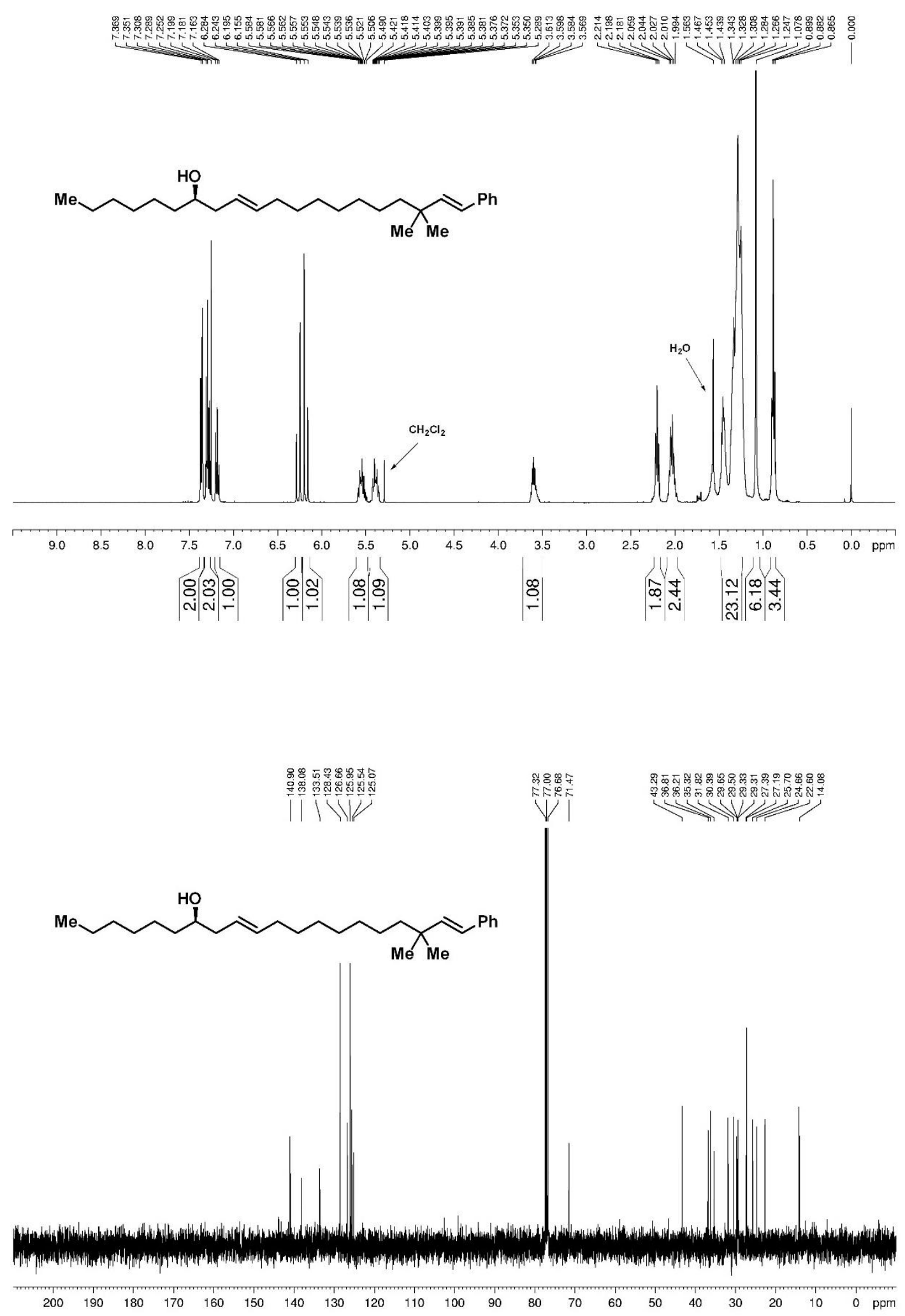
3bb; ${ }^{1} \mathrm{H}$ NMR (600 MHz, $\left.\mathrm{CDCl}_{3}\right) ;{ }^{13} \mathrm{C} \mathrm{NMR}\left(150 \mathrm{MHz}, \mathrm{CDCl}_{3}\right)$
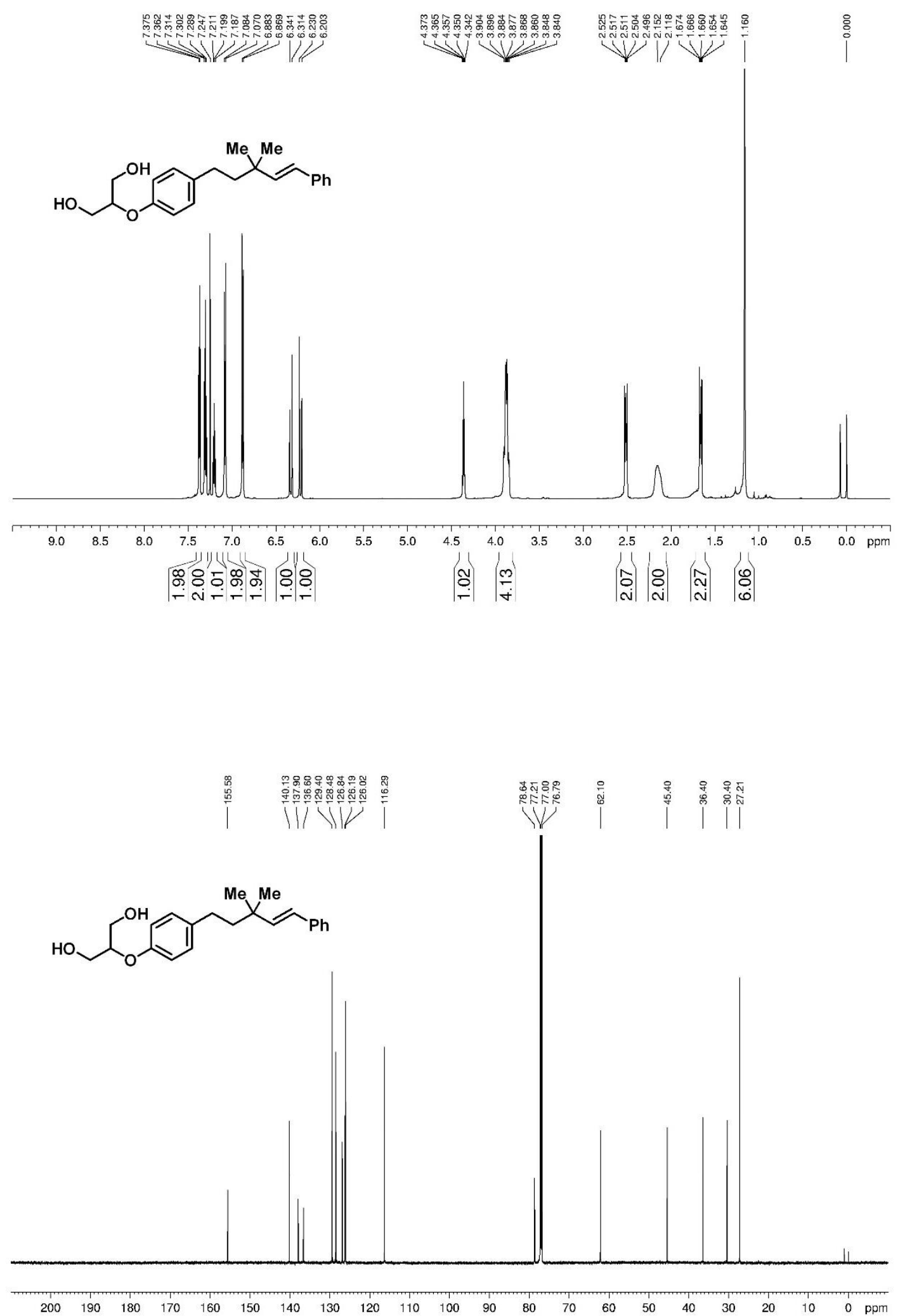
3bc; ${ }^{1} \mathrm{H}$ NMR (600 MHz, $\left.\mathrm{CDCl}_{3}\right) ;{ }^{13} \mathrm{C} \mathrm{NMR}\left(150 \mathrm{MHz}, \mathrm{CDCl}_{3}\right)$
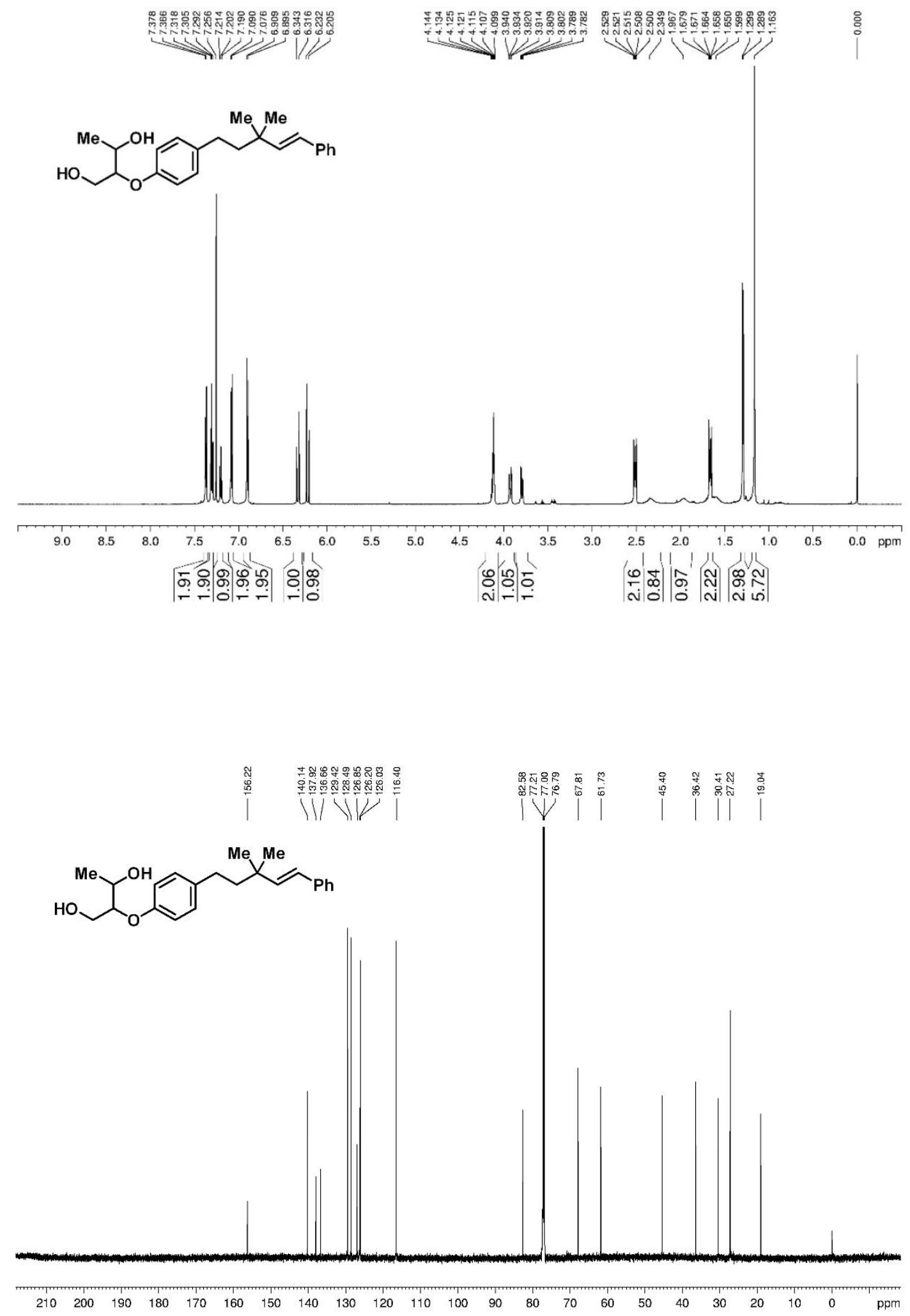
3bd; ${ }^{1} \mathrm{H}$ NMR (600 MHz, MeOD); ${ }^{13} \mathrm{C}$ NMR (150 MHz, MeOD)
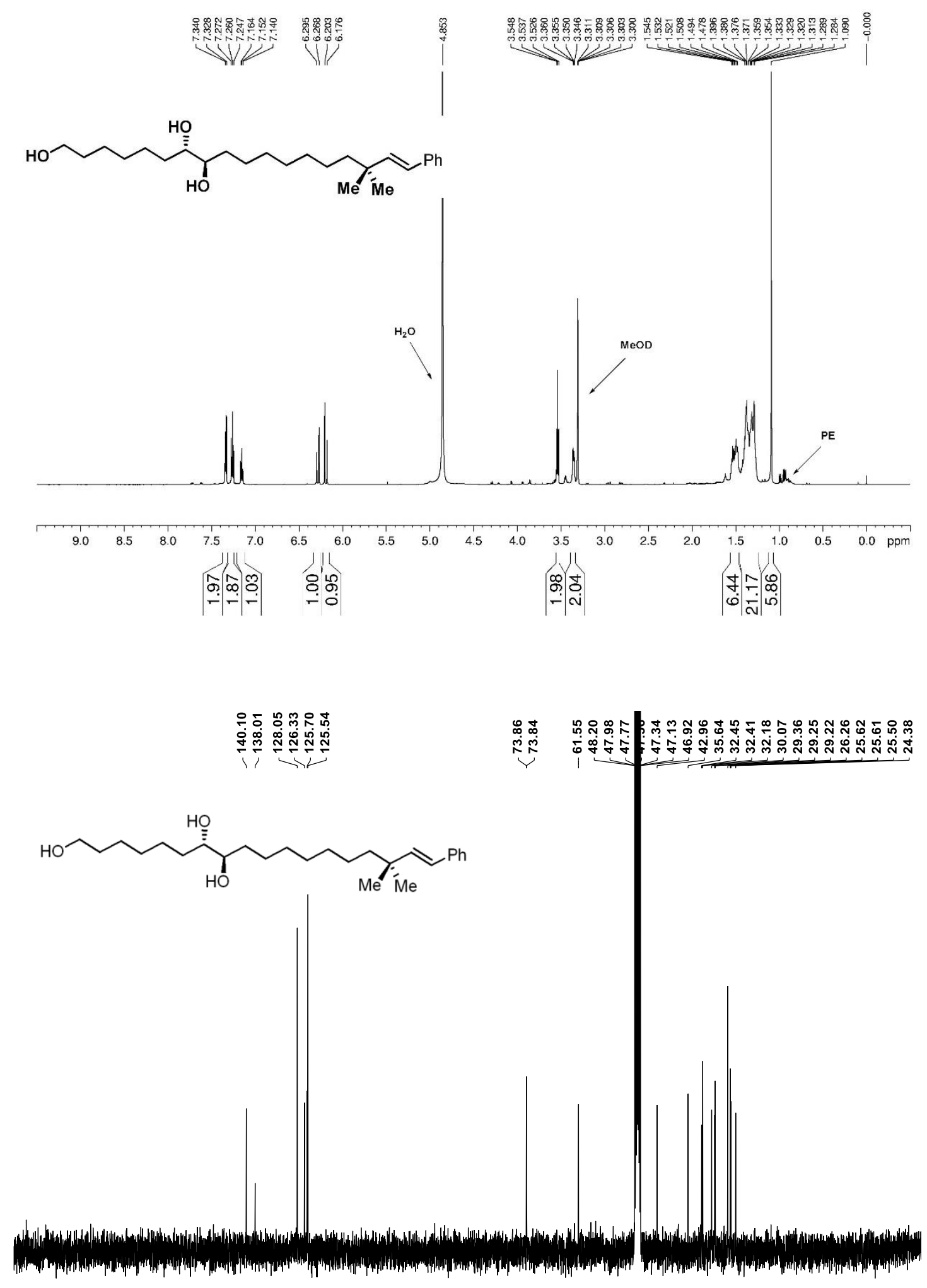

$\begin{array}{llllllllllllllllllll}190 & 180 & 170 & 160 & 150 & 140 & 130 & 120 & 110 & 100 \underset{\mathrm{f} 1}{90}(\mathrm{ppm}) & 70 & 60 & 50 & 40 & 30 & 20 & 10 & 0 & -10\end{array}$ 
3be; ${ }^{1} \mathrm{H}$ NMR (600 MHz, $\left.\mathrm{CDCl}_{3}\right) ;{ }^{13} \mathrm{C}$ NMR (150 MHz, CDCl 3$)$
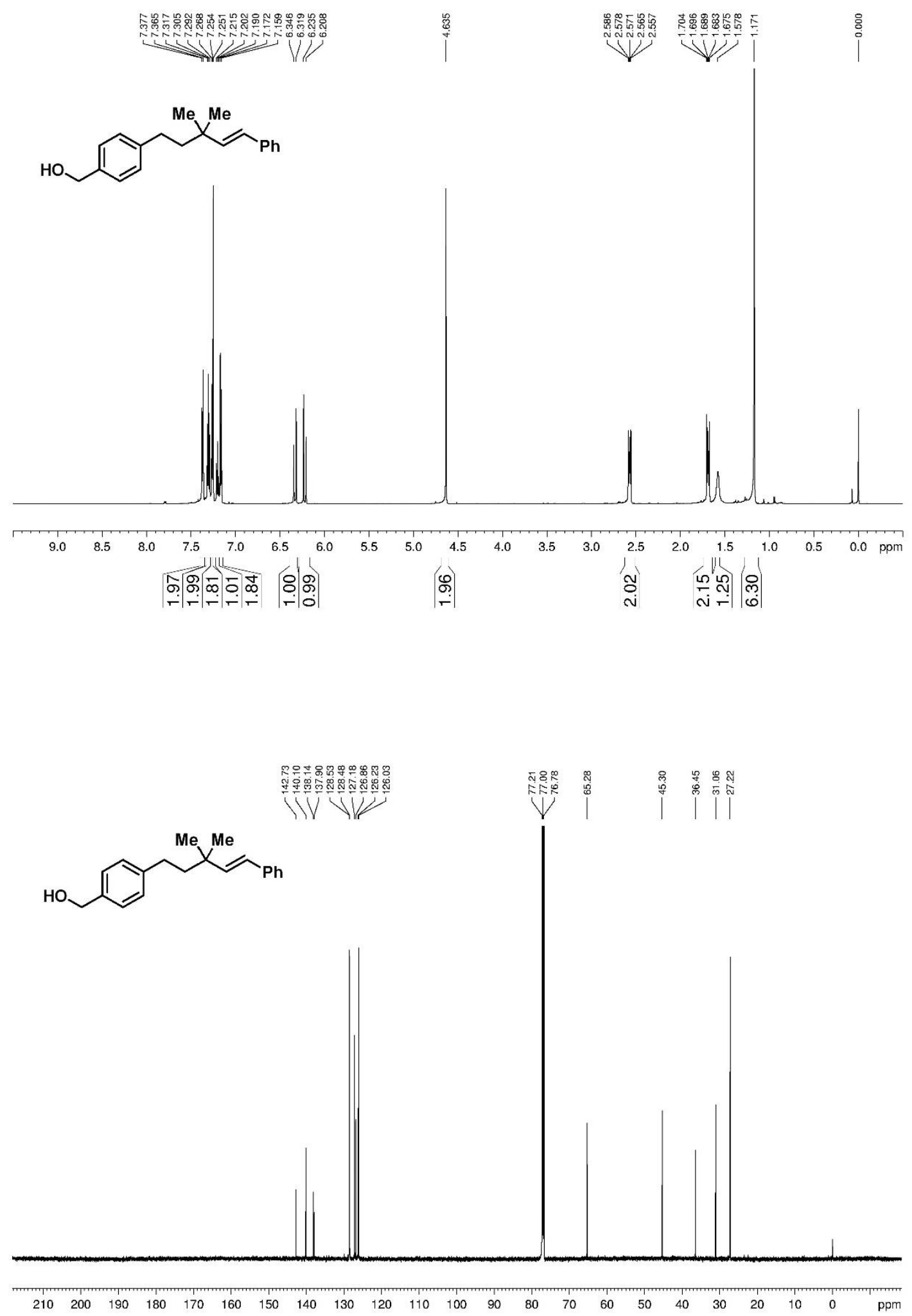
3bf; ${ }^{1} \mathrm{H}$ NMR (600 MHz, MeOD); ${ }^{13} \mathrm{C}$ NMR (150 MHz, MeOD)

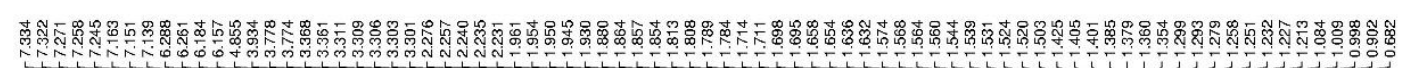
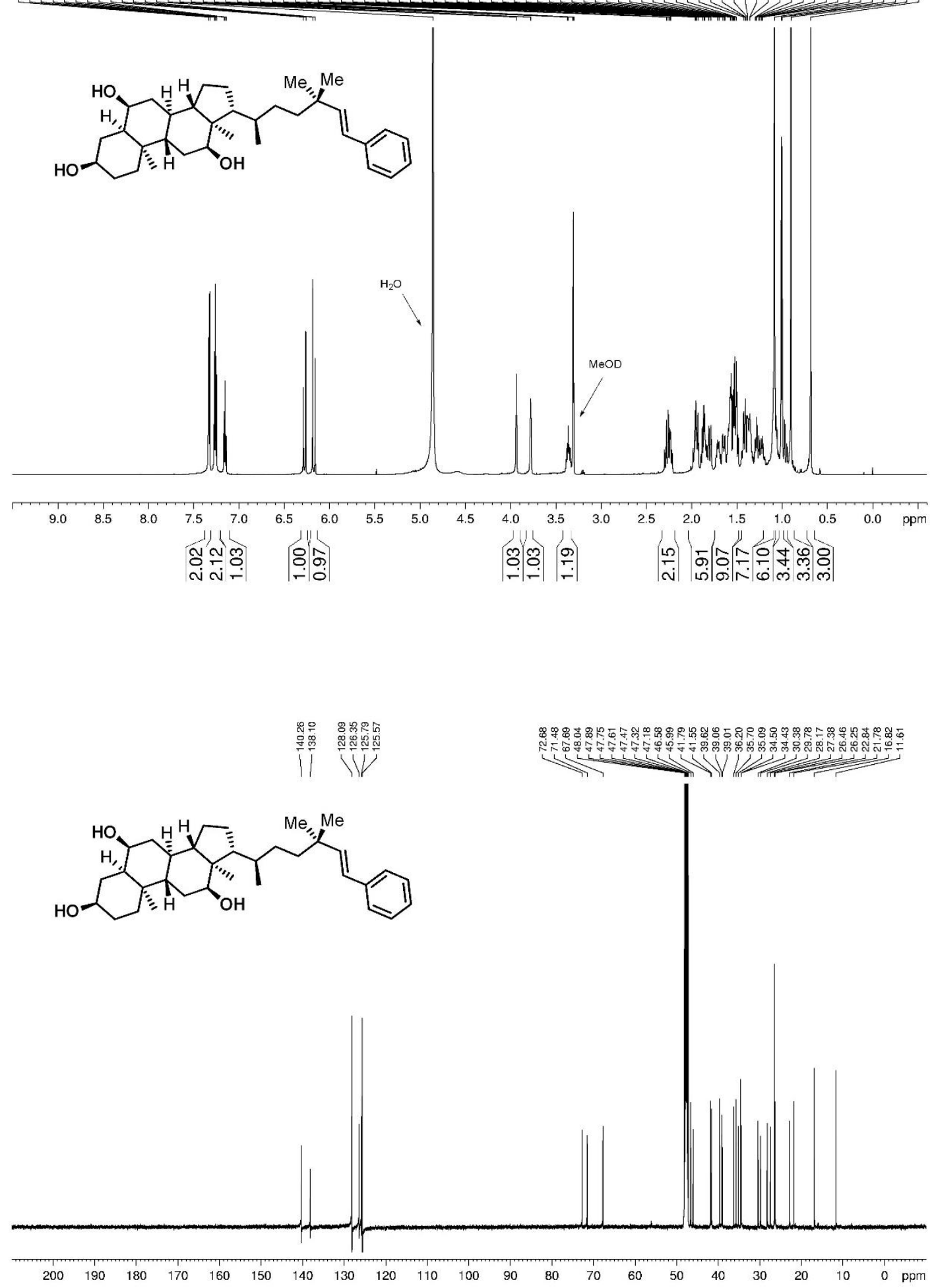
6; ${ }^{1} \mathrm{H}$ NMR (400 MHz, $\left.\mathrm{CDCl}_{3}\right) ;{ }^{13} \mathrm{C}$ NMR (100 MHz, $\left.\mathrm{CDCl}_{3}\right)$
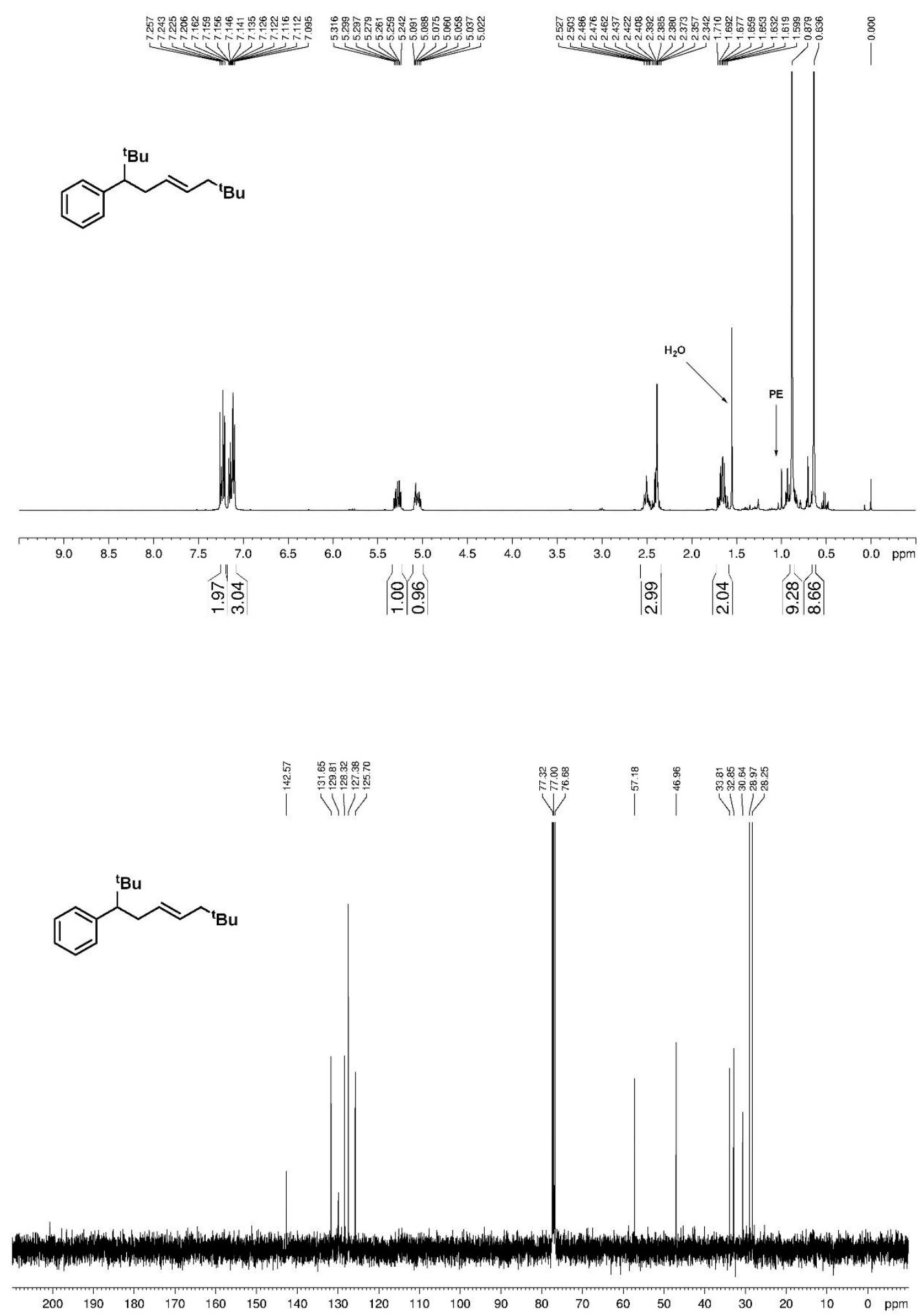
7; ${ }^{1} \mathrm{H}$ NMR (600 MHz, $\left.\mathrm{CDCl}_{3}\right) ;{ }^{13} \mathrm{C}$ NMR (150 MHz, $\left.\mathrm{CDCl}_{3}\right)$
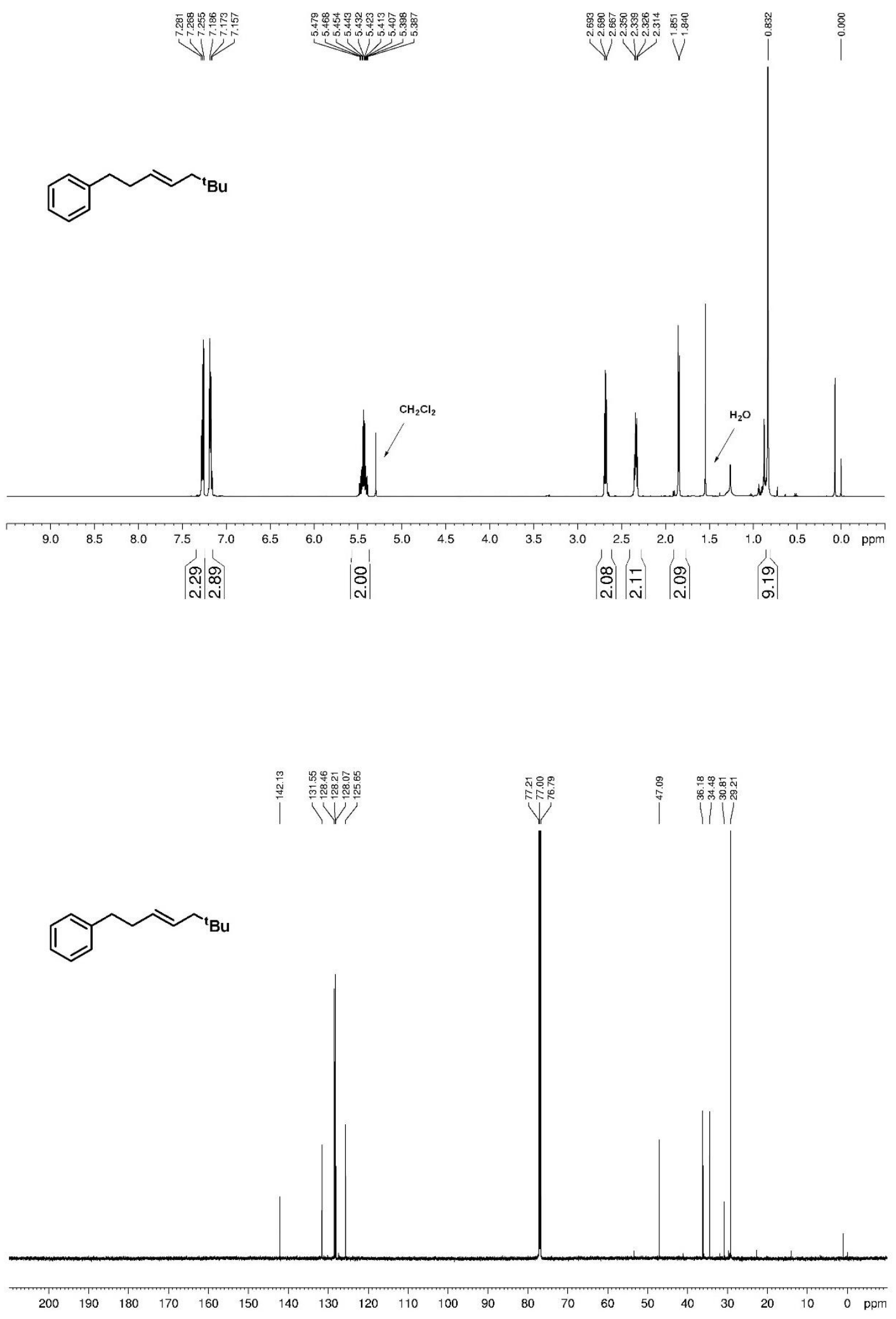
10; ${ }^{1} \mathrm{H}$ NMR (400 MHz, CDCl $) ;{ }^{13} \mathrm{C}$ NMR (100 MHz, CDCl 3$)$
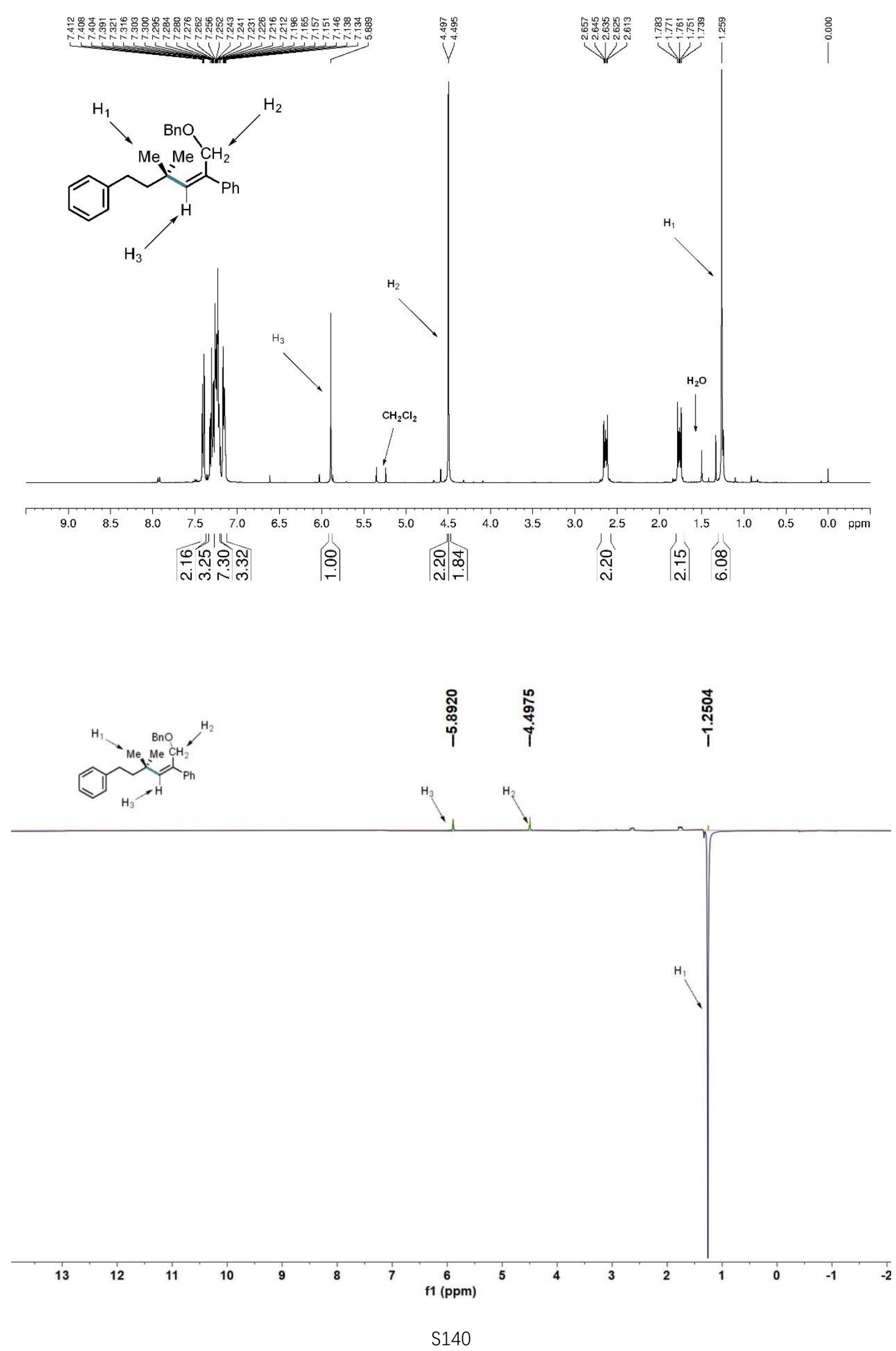


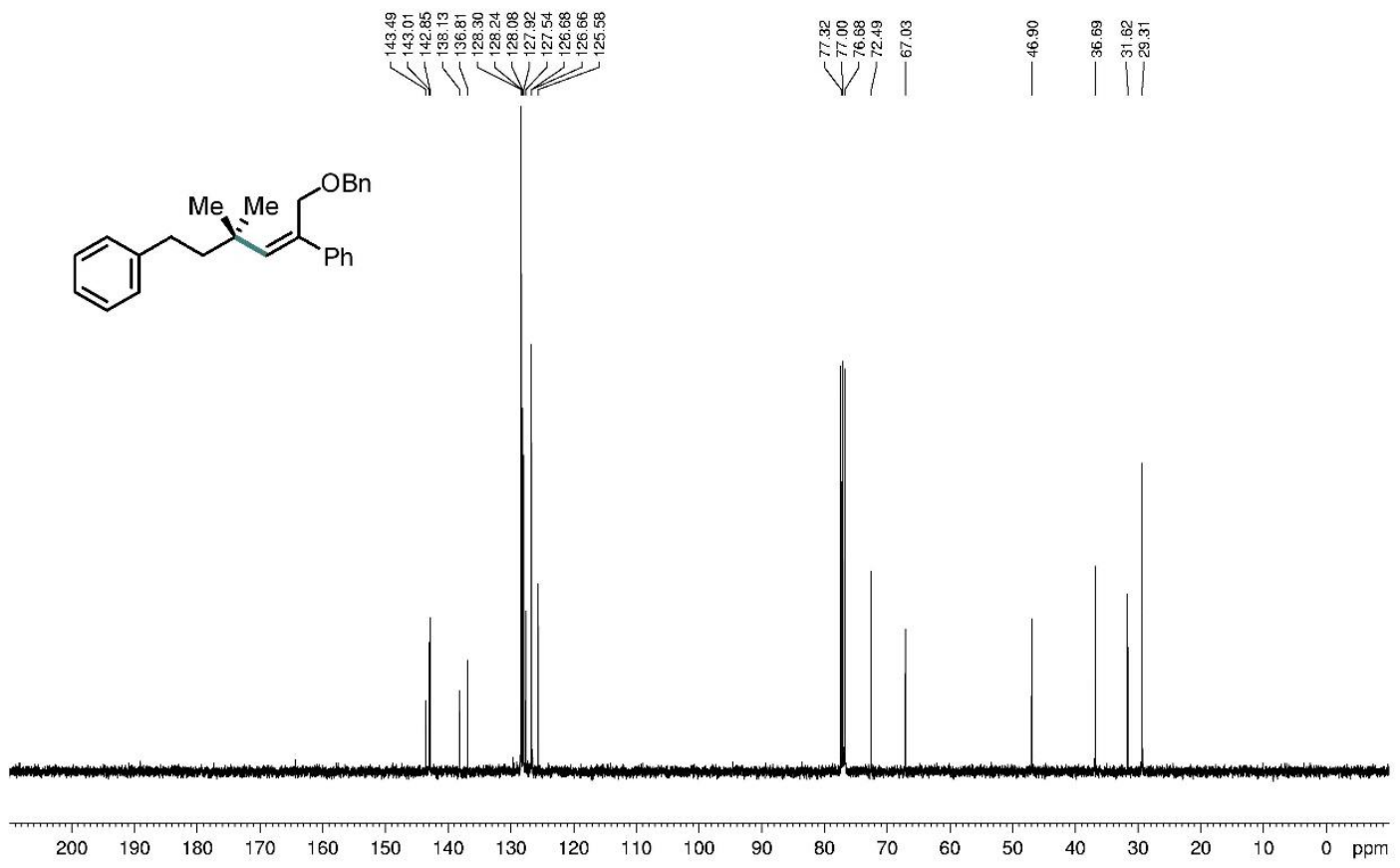


11; ${ }^{1} \mathrm{H}$ NMR (400 MHz, CDCl3); ${ }^{13} \mathrm{C}$ NMR (100 MHz, CDCl $)$
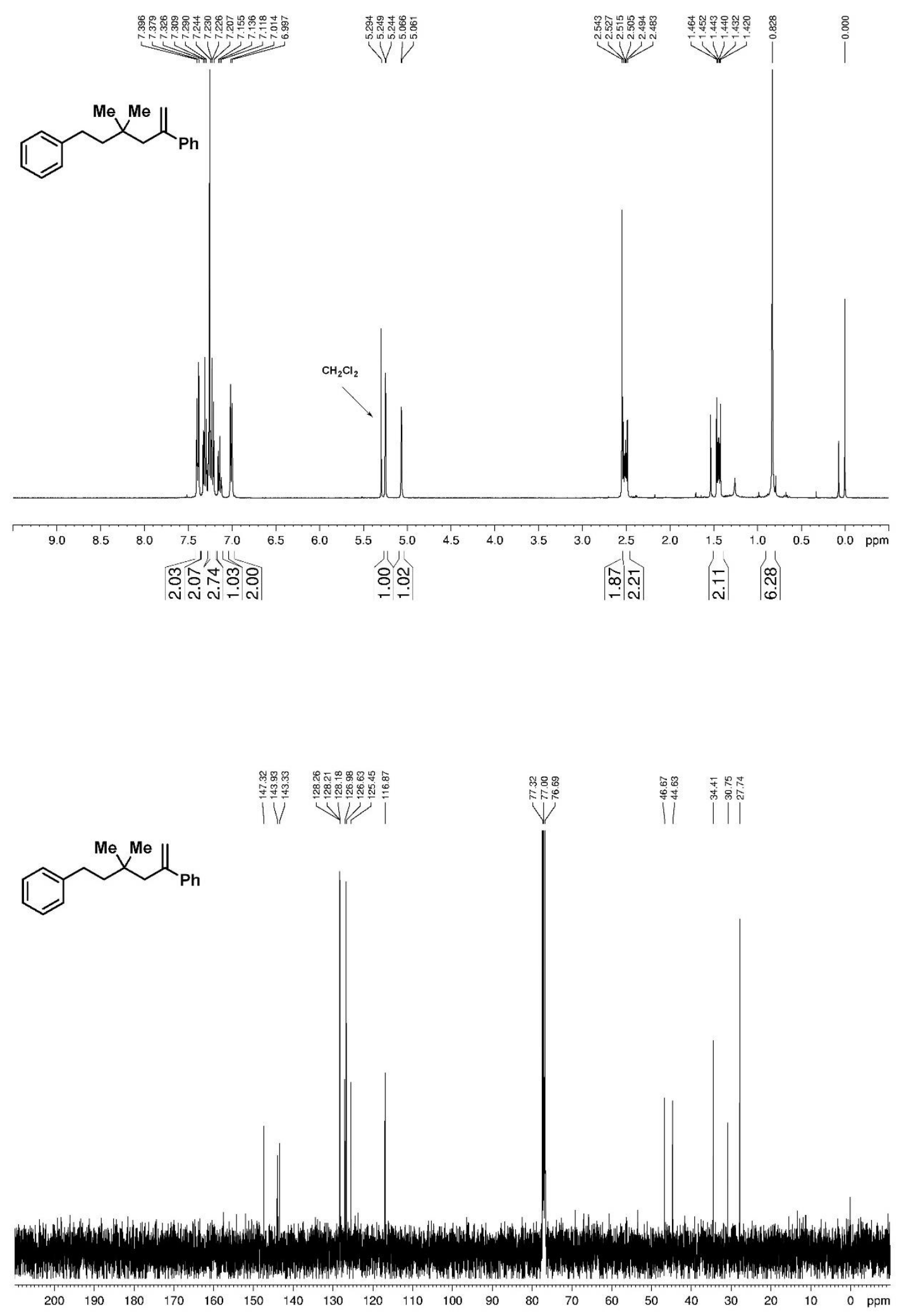